\title{
A Versatile New Reagent for Nitrosation under Mild Conditions
}

Jordan D. Galloway ${ }^{1}$, Cristian Sarabia ${ }^{1}$, James C. Fettinger ${ }^{2}$, Hrant P. Hratchian ${ }^{1}$, and Ryan D. Baxter $^{1 *}$

${ }^{1}$ Department of Chemistry and Chemical Biology, University of California, Merced. Merced, California 95343, United States. ${ }^{2}$ Department of Chemistry, University of California, Davis. Davis, California 95616.

*corresponding author: rbaxter@ucmerced.edu

\section{Supporting Information}

Experimental procedures, characterization data for reported compounds, and copies of NMR spectra

\section{Table of Contents}

General Considerations and Reaction Procedures

Experimental References

Spectroscopic Data for Reported Compounds

Calculation Setup

Optimized Geometries

Computational Citations

Crystal Sample Preparation and Structure Determination

Crystal Atomic Coordinates

Crystallographic References

Probability Ellipsoids (50\%) 
General Considerations. Reagents and solvents were purchased and used without purification. Yields refer to homogenous material that is purified by silica-gel chromatography and spectroscopically pure (>95\%) by ${ }^{1} \mathrm{H}$ NMR and $13 \mathrm{C}$ NMR. Yields in the Supporting Information describe the result of a single experiment, whereas the yields reported in Schemes 1-3 are an average of two or more experiments. Reactions were monitored by thin-layer chromatography using $0.25 \mathrm{~mm}$ E. Merck silica gel plates (60F-254). NMR spectra were recorded on a VarianINOVA $400 \mathrm{Mhz}$ or $500 \mathrm{Mhz}$ spectrometer, calibrated using residual undeuterated solvent as an internal reference $\left(\mathrm{CDCl}_{3}-{ }^{1} \mathrm{H}\right.$ NMR $\left.7.26 \mathrm{ppm},{ }^{13} \mathrm{C} \mathrm{NMR}-77.16 \mathrm{ppm}\right)$. The following abbreviations were used to explain multiplicities (s-singlet, $d$-doublet, $t$-triplet, q-quartet, $\mathrm{m}$ multiplet).

Procedure for synthesis of NO-1.

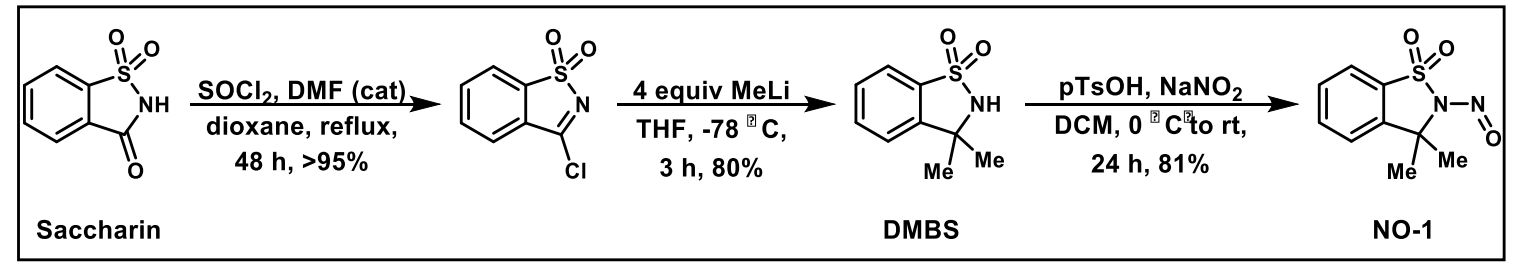<smiles>O=S1(=O)N=C(Cl)c2ccccc21</smiles>

3-chlorobenzo[d] isothiazole 1,1-dioxide

3-chlorobenzo[d]isothiazole 1,1-dioxide. To a $500 \mathrm{~mL}$ round bottom with a stir bar atop a

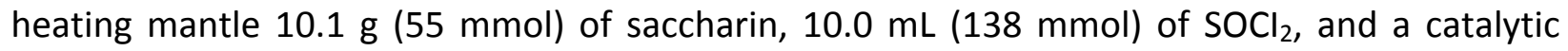
amount of DMF $(1 \mathrm{ml})$ was added in $250 \mathrm{~mL}$ of 1,4-dioxane. The reaction was heated for 48 hours at reflux. The clear brown solution was concentrated in vacuo in a rotary evaporator in a heating bath. The residue was used in the next step without further purification. The data matches those previously reported. ${ }^{[1]}$

${ }^{1} \mathrm{H}$ NMR (500 MHz, $\left.\mathrm{CDCl}_{3}\right): 7.93(\mathrm{~d}, J=7.3 \mathrm{~Hz}, 1 \mathrm{H}), 7.91-7.80(\mathrm{~m}, 3 \mathrm{H})$.

${ }^{13} \mathrm{C}$ NMR (125 MHz, $\left.\mathrm{CDCl}_{3}\right)$ : 166.3, 140.6, 135.1, 134.6, 129.9, 125.3, 122.6. 


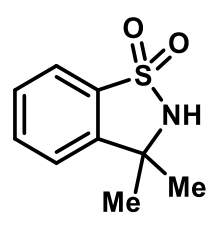

DMBS

3,3-dimethyl-2,3-dihydrobenzo[d]isothiazole 1,1-dioxide (DMBS). To a $250 \mathrm{~mL}$ round bottom $100 \mathrm{~mL}$ of dry diethyl ether was added to $11 \mathrm{~g}$ of 3-chlorobenzo[d]isothiazole 1,1-dioxide (55 $\mathrm{mmol})$. The reaction was cooled to $-10{ }^{\circ} \mathrm{C}$. Slowly added to the chilled solution was 4 equivalents of $1.6 \mathrm{M} \mathrm{MeLi}$. The reaction was stirred for $30 \mathrm{~min}$ at $-10^{\circ} \mathrm{C}$. The reaction was warmed to RT and allowed to stir for $2.5 \mathrm{~h}$. Once reaction is complete pour into $150 \mathrm{~mL}$ of dilute $\mathrm{HCL}(5 \%)$. The organic layer was separated and washed with water until neutral. The crude reaction was absorbed onto silica and purified with EtOAc/Hexanes $(50 / 50) . R_{f}=0.4$. The product isolated as a pale yellow solid in $9.9 \mathrm{~g}(80 \%$ yield). The data matches those previously reported. ${ }^{[2]}$

${ }^{1} \mathrm{H}$ NMR $\left(500 \mathrm{MHz}, \mathrm{CDCl}_{3}\right): 7.63(\mathrm{~d}, J=7.8 \mathrm{~Hz}, 1 \mathrm{H}), 7.57-7.52(\mathrm{~m}, 1 \mathrm{H}), 7.44-7.38(\mathrm{~m}, 1 \mathrm{H}), 7.34$ $(\mathrm{d}, J=7.8 \mathrm{~Hz}, 1 \mathrm{H}), 5.42(\mathrm{~s}, 1 \mathrm{H}), 1.56(\mathrm{~s}, 6 \mathrm{H})$.

${ }^{13}$ C NMR (125 MHz, CDCl $)$ ): 146.0, 134.8, 133.3, 128.9, 122.8, 120.8, 60.8, 29.4.

HRMS (ESI-TOF) m/z: [M + H]+ Calcd for $\mathrm{C}_{9} \mathrm{H}_{11} \mathrm{NO}_{2} \mathrm{~S}[\mathrm{M}+\mathrm{H}]^{+} 198.0583$ found 198.05074 .

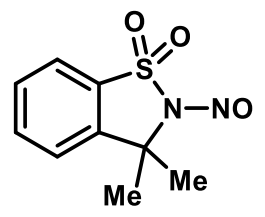

NO-1

3,3-dimethyl-2-nitroso-2,3-dihydrobenzo[d]isothiazole 1,1-dioxide (NO-1). To a large $1 \mathrm{~L}$ round bottom $10 \mathrm{~g}(0.50 \mathrm{~mol})$ of DMBS was added with $250 \mathrm{~mL}$ of DCM and $8.6 \mathrm{~g}(70 \mathrm{mmol})$ of $p$ TsOH. The mixture was stirred and cooled to $0{ }^{\circ} \mathrm{C}$. Slowly added was $5.0 \mathrm{~g}(72 \mathrm{mmol}) \mathrm{NaNO}_{2}$. The reaction was left to warm to room temperature and stirred overnight. The was filtered through a Büchner funnel to remove the insoluble material. The filtrate was then absorbed onto silica and purified with column chromatography using DCM $(\mathrm{Rf}=0.4)$ or $10 \%$ EtOAc in hexanes as the mobile phase. Isolated was $8.9 \mathrm{~g}$ ( $81 \%$ yield) of greenish yellow solid with melting points of $70^{\circ} \mathrm{C}$ and $90^{\circ} \mathrm{C}$ (NO-1a and NO-1b, respectively).

${ }^{1} \mathrm{H}$ NMR $\left(500 \mathrm{MHz}, \mathrm{CDCl}_{3}\right): 7.88-7.75(\mathrm{~m}, 2 \mathrm{H}), 7.64(\mathrm{t}, J=7.7 \mathrm{~Hz}, 1 \mathrm{H}), 7.58(\mathrm{~d}, J=7.8 \mathrm{~Hz}, 1 \mathrm{H})$, $1.99(\mathrm{~s}, 6 \mathrm{H})$.

${ }^{13} \mathrm{C}$ NMR (125 MHz, CDCl 3$): 135.5,130.3,123.5,122.0,65.5,29.6$.

HRMS (ESI-TOF) m/z: [M + H]+ Calcd for $\mathrm{C}_{9} \mathrm{H}_{10} \mathrm{~N}_{2} \mathrm{O}_{3} \mathrm{~S}[\mathrm{M}+\mathrm{H}]^{+} 227.0485$ found 227.0482 . 
Differential Scanning Calorimetry

DSC NO-1

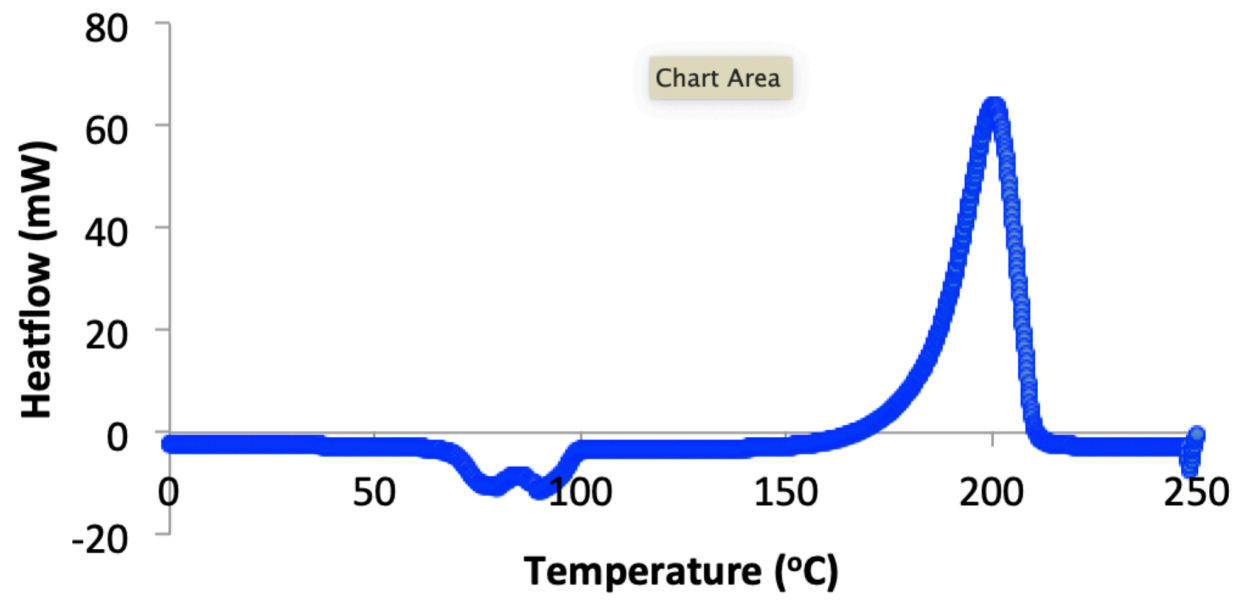

Figure S1. An endothermic response is noted at approximately $70^{\circ} \mathrm{C}$ to indicate an initial melting point. A second endothermic response is also noted at approximately $90^{\circ} \mathrm{C}$, likely representing separate melting points for the individual rotamers. The onset of a large exotherm is noted at approximately $180^{\circ} \mathrm{C}$, indicating thermal decomposition of NO-1.

\section{Thermogravimetric Analysis}

\section{TGA NO-1}

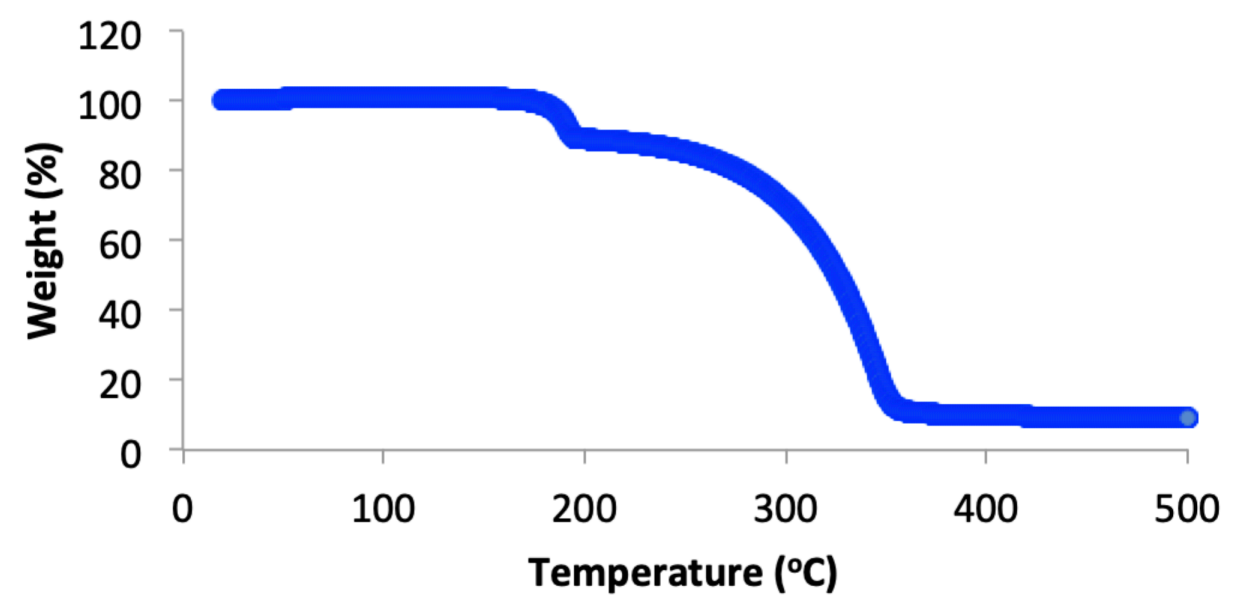

Figure S2. Thermogravimetric analysis shows that NO-1 is stable up to $180^{\circ} \mathrm{C}$. Decomposition is noted to begin between $180^{\circ} \mathrm{C}-200^{\circ} \mathrm{C}$, leading to nearly total mass loss by $360^{\circ} \mathrm{C}$. 


\section{Reaction Procedures.}

Safety. Nitroso compounds, especially alkyl nitrites are known to decompose in air to form explosive mixtures. In the case for crystalline solids isolated, the melting point was not reported out of a safety precaution.

[A] General Procedure for Nitrosation. The threads of a $3 \mathrm{~mL}$ borosilicate scintillation vial were thoroughly taped with Teflon tape. To this vial containing a stir bar was added substrate $(0.2$ mmol, 1 equiv), and NO-1 (54.0 mg, $0.24 \mathrm{mmol}, 1.2$ equiv). Dichloromethane $(2 \mathrm{~mL})$ was then added and the reaction was stirred at room temperature on a stir plate until completed (thinlayer chromatography). In some reactions when heating is required $\left(80^{\circ} \mathrm{C}\right)$ the vials are stired on a hotplate and dichloroethane $(2 \mathrm{~mL})$ or acetonitrile $(2 \mathrm{~mL})$ was used in replacement of dichloromethane.

Upon completion, the solvent was removed under reduced pressure, crude mixture was directly absorbed onto silica and purified by silica gel chromatography to yield the desired product.

[B] General Procedure for Acid Catalyzed Nitrosation. The threads of a $3 \mathrm{~mL}$ borosilicate scintillation vial were thoroughly taped with Teflon tape. To this vial containing a stir bar was added substrate ( $0.2 \mathrm{mmol}, 1$ equiv), and NO-1 (54.0 mg, $0.24 \mathrm{mmol}, 1.2$ equiv). Acetonitrile ( 2 $\mathrm{mL})$ and trifluoracetic acid $(3.1 \mu \mathrm{L}, 20 \mathrm{~mol} \%)$ was then added and the reaction was stirred at room temperature until completed (thin-layer chromatography).

Upon completion, the solvent was removed under reduced pressure, crude mixture was directly absorbed onto silica and purified by silica gel chromatography to yield the desired product.

Scheme 1 Compounds (1-14)

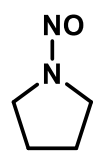

1-nitrosopyrrolidine (1). General procedure A was employed using pyrrolidine (17 $\mu \mathrm{L}, 0.2$ $\mathrm{mmol})$ in dichloromethane. The reaction afforded $1(18.6 \mathrm{mg}, 93 \%$ yield) as a pale yellow oil separated by silica gel (30\% ethyl acetate in hexanes). The data matches those previously reported. ${ }^{[3]}$

${ }^{1} \mathrm{H}$ NMR $\left(500 \mathrm{MHz}, \mathrm{CDCl}_{3}\right): 4.17(\mathrm{t}, J=6.8 \mathrm{~Hz}, 1 \mathrm{H}), 3.48(\mathrm{t}, J=7.1 \mathrm{~Hz}, 1 \mathrm{H}), 2.04-1.88(\mathrm{~m}, 2 \mathrm{H})$.

${ }^{13} \mathrm{C}$ NMR (125 MHz, CDCl 3$): 49.7,45.1,23.9,22.5$.

HRMS (ESI-TOF) m/z: [M + H]+ Calcd for $\mathrm{C}_{4} \mathrm{H}_{9} \mathrm{~N}_{2} \mathrm{O} 101.0709$ found 101.0707. 
<smiles>O=[N+]1CCOCC1</smiles>

4-nitrosomorpholine (2). General procedure A was employed using morpholine (17 mg, 0.2 $\mathrm{mmol})$. The reaction afforded $\mathbf{2}(21.1 \mathrm{mg}, 91 \%$ yield) as a pale yellow oil separated by silica gel (30\% ethyl acetate in hexanes). The data matches those previously reported. ${ }^{[3]}$

${ }^{1}$ H NMR (500 MHz, CDCl $)$ ): $4.33-4.25(\mathrm{~m}, 1 \mathrm{H}), 3.92-3.83(\mathrm{~m}, 2 \mathrm{H}), 3.65(\mathrm{t}, \mathrm{J}=5.2 \mathrm{~Hz}, 1 \mathrm{H})$.

${ }^{13} \mathrm{C}$ NMR (125 MHz, $\left.\mathrm{CDCl}_{3}\right): 67.4,66.0,50.1,40.5$.

HRMS (ESI-TOF) m/z: [M + H]+ Calcd for $\mathrm{C}_{4} \mathrm{H}_{9} \mathrm{~N}_{2} \mathrm{O}_{2} 117.0659$ found 117.0656 .<smiles>O=[N+]([O-])N1CCNCC1</smiles>

1-nitrosopiperazine (3). General procedure A was employed using piperazine (17 $\mu \mathrm{L}, 0.2 \mathrm{mmol})$. The reaction afforded 3 ( $22.5 \mathrm{mg}, 98 \%$ yield) as a pale yellow oil separated by silica gel (40\% ethyl acetate in hexanes). The data matches those previously reported. ${ }^{[4]}$

${ }^{1} \mathrm{H}$ NMR (500 MHz, $\left.\mathrm{CDCl}_{3}\right): 4.27-4.18(\mathrm{~m}, 1 \mathrm{H}), 3.85-3.78(\mathrm{~m}, 1 \mathrm{H}), 3.11-3.03(\mathrm{~m}, 1 \mathrm{H}), 2.87-$ $2.78(\mathrm{~m}, 1 \mathrm{H}), 2.67(\mathrm{~s}, 1 \mathrm{H})$.

${ }^{13} \mathrm{C}$ NMR (125 MHz, $\left.\mathrm{CDCl}_{3}\right): 51.1,46.6,45.1,40.8$.

HRMS (ESI-TOF) m/z: [M + H]+ Calcd for $\mathrm{C}_{4} \mathrm{H}_{10} \mathrm{~N}_{3} \mathrm{O} 116.0818$ found 116.0815 .

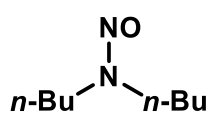

4

$\mathbf{N}, \mathbf{N}$-dibutylnitrous amide (4). General procedure A was employed using dibutylamine (36 $\mu \mathrm{L}$, $0.2 \mathrm{mmol})$. The reaction afforded $4(30.7 \mathrm{mg}, 97 \%$ yield) as a yellow oil separated by silica gel (40\% ethyl acetate in hexanes). The data matches those previously reported. ${ }^{[3]}$

${ }^{1} \mathrm{H}$ NMR (500 MHz, $\left.\mathrm{CDCl}_{3}\right): 4.06(\mathrm{t}, J=7.3 \mathrm{~Hz}, 2 \mathrm{H}), 3.58-3.48(\mathrm{~m}, 2 \mathrm{H}), 1.77-1.66(\mathrm{~m}, 2 \mathrm{H}), 1.49$ $-1.41(\mathrm{~m}, 2 \mathrm{H}), 1.41-1.33(\mathrm{~m}, 2 \mathrm{H}), 1.32-1.23(\mathrm{~m}, 2 \mathrm{H}), 0.95(\mathrm{t}, J=7.4 \mathrm{~Hz}, 3 \mathrm{H}), 0.90(\mathrm{t}, J=7.3$ $\mathrm{Hz}, 3 \mathrm{H})$.

${ }^{13} \mathrm{C}$ NMR (125 MHz, CDCl 3$): 52.1,43.6,30.4,28.2,20.5,19.9,13.8,13.7$. HRMS (ESI-TOF) m/z: [M + H]+ Calcd for $\mathrm{C}_{10} \mathrm{H}_{12} \mathrm{NO}_{4} 159.1492$ found 159.1486. 


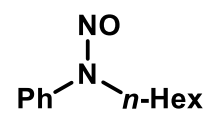

$\mathrm{N}$-hexyl-N-phenylnitrous amide (5). General procedure A was employed using N-hexylaniline $(40 \mu \mathrm{L}, 0.2 \mathrm{mmol})$. The reaction afforded 5 ( $41.0 \mathrm{mg}, 93 \%$ yield) as a pale yellow oil separated by silica gel (10\% DCM in hexanes).

${ }^{1} \mathrm{H}$ NMR $\left(500 \mathrm{MHz}, \mathrm{CDCl}_{3}\right): 7.53(\mathrm{~d}, \mathrm{~J}=7.6 \mathrm{~Hz}, 2 \mathrm{H}), 7.47(\mathrm{t}, \mathrm{J}=7.4 \mathrm{~Hz}, 2 \mathrm{H}), 7.36(\mathrm{t}, \mathrm{J}=6.9 \mathrm{~Hz}, 1 \mathrm{H})$, $4.08-3.95(\mathrm{~m}, 2 \mathrm{H}), 1.54(\mathrm{~s}, 2 \mathrm{H}), 1.27(\mathrm{~s}, 6 \mathrm{H}), 0.86(\mathrm{~s}, 3 \mathrm{H})$.

${ }^{13} \mathrm{C}$ NMR (125 MHz, CDCl 3$): 141.8,129.6,127.4,119.8,44.1,31.4,26.8,26.6,22.6,14.1$. HRMS (ESI-TOF) m/z: [M + H]+ Calcd for $\mathrm{C}_{12} \mathrm{H}_{19} \mathrm{~N}_{2} \mathrm{O} 207.1492$ found 207.1491.

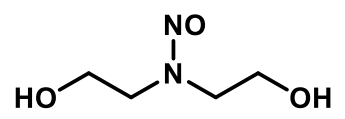

6

N,N-bis(2-hydroxyethyl)nitrous amide (6). General procedure A was employed using diethanolamine $(19 \mu \mathrm{L}, 0.2 \mathrm{mmol})$. The reaction afforded $6(24.4 \mathrm{mg}, 91 \%$ yield) as a pale yellow oil separated by silica gel (10\% $\mathrm{MeOH}$ in DCM). The data matches those previously reported. ${ }^{[3]}$

${ }^{1} \mathrm{H}$ NMR $\left(500 \mathrm{MHz},\left(\mathrm{CD}_{3}\right)_{2} \mathrm{SO}\right): 4.92(\mathrm{t}, J=5.5 \mathrm{~Hz}, 1 \mathrm{H}), 4.85(\mathrm{t}, J=5.5 \mathrm{~Hz}, 1 \mathrm{H}), 4.18(\mathrm{t}, J=5.6 \mathrm{~Hz}$, $2 \mathrm{H}), 3.72(\mathrm{q}, J=5.5 \mathrm{~Hz}, 2 \mathrm{H}), 3.66(\mathrm{t}, J=6.0 \mathrm{~Hz}, 2 \mathrm{H}), 3.44(\mathrm{q}, J=5.8 \mathrm{~Hz}, 2 \mathrm{H})$.

${ }^{13} \mathrm{C}$ NMR (125 MHz, $\left.\left(\mathrm{CD}_{3}\right)_{2} \mathrm{SO}\right): 58.7,56.8,55.1,46.5$.

HRMS (ESI-TOF) m/z: [M + H]+ Calcd for $\mathrm{C}_{4} \mathrm{H}_{11} \mathrm{~N}_{2} \mathrm{O}_{3} 135.0764$ found 135.0797 .

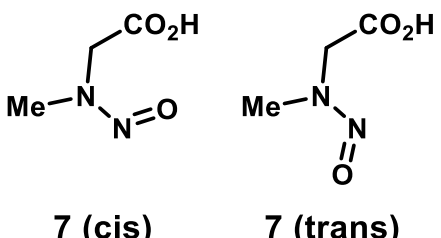

N-methyl-N-nitrosoglycine (7). General procedure A was employed using Sarcosine (18 mg, 0.2 $\mathrm{mmol}$ ) in dichloroethane. The reaction afforded $\mathbf{7}$ (trans) and $\mathbf{7}$ (cis) as a mixture of rotamers in a 1:1 ratio ( $21.2 \mathrm{mg}, 90 \%$ yield) as a pale yellow oil separated by silica gel (10\% $\mathrm{MeOH}$ in DCM).

(7 trans) and (7 cis)

${ }^{1} \mathrm{H}$ NMR $\left(500 \mathrm{MHz}, \mathrm{CDCl}_{3}\right): 10.25(\mathrm{~s}, 1 \mathrm{H}), 5.02(\mathrm{~s}, 2 \mathrm{H}), 4.31(\mathrm{~s}, 2 \mathrm{H}), 3.91(\mathrm{~s}, 3 \mathrm{H}), 3.14(\mathrm{~s}, 3 \mathrm{H})$.

${ }^{13} \mathrm{C}$ NMR (125 MHz, $\left.\mathrm{CDCl}_{3}\right): 172.6,170.7,54.4,46.7,40.1,32.9$.

HRMS (ESI-TOF) m/z: [M + H]+ Calcd for $\mathrm{C}_{3} \mathrm{H}_{7} \mathrm{~N}_{2} \mathrm{O}_{3} 119.0451$ found 119.0448 . 


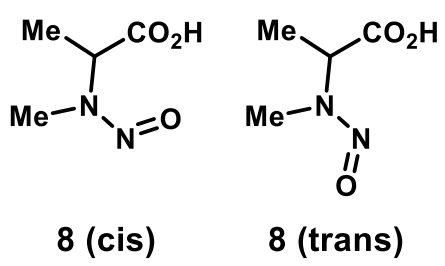

N-methyl-N-nitrosoalanine. General procedure A was employed using DL-Alanine (21 mg, 0.2 $\mathrm{mmol}$ ) in dichloroethane. The reaction afforded $\mathbf{8 ( t r a n s )}$ and $\mathbf{8 ( c i s )}$ as a mixture of rotamers in a $1: 3$ ratio ( $25.6 \mathrm{mg}, 97 \%$ yield) as a pale yellow oil separated by silica gel ( $100 \%$ ethyl acetate).

(8 trans) and (8 cis)

${ }^{1} \mathrm{H}$ NMR $\left(500 \mathrm{MHz} \mathrm{CDCl}_{3}\right): 11.70(\mathrm{~s}, 1 \mathrm{H}), 5.47(\mathrm{q}, J=7.0 \mathrm{~Hz}, 1 \mathrm{H}), 5.10(q, J=7.0 \mathrm{~Hz}, 1 \mathrm{H}), 3.81(\mathrm{~s}$, $3 \mathrm{H}), 3.05(\mathrm{~s}, 3 \mathrm{H}), 1.71(\mathrm{~d}, J=7.1 \mathrm{~Hz}, 3 \mathrm{H}), 1.46(\mathrm{~d}, J=7.0 \mathrm{~Hz}, 3 \mathrm{H})$.

${ }^{13} \mathrm{C}$ NMR (125 MHz, $\left.\mathrm{CDCl}_{3}\right):$ 174.4, 173.5, 61.0, 52.0, 36.7, 30.3, 15.5, 12.9.

HRMS (ESI-TOF) m/z: [M + H]+ Calcd for $\mathrm{C}_{4} \mathrm{H}_{9} \mathrm{~N}_{2} \mathrm{O}_{3} 133.0608$ found 133.0604 .

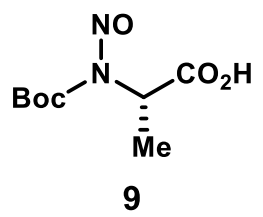

$\mathbf{N}$-(tert-butoxycarbonyl)-N-nitroso-L-alanine (9). General procedure B was employed using Boc-Ala-OH (38 mg, $0.2 \mathrm{mmol})$. The reaction afforded 9 (31.8 $\mathrm{mg}, 73 \%$ yield) as a pale yellow oil separated by silica gel (5-10\% $\mathrm{MeOH}$ in DCM).

${ }^{1} \mathrm{H}$ NMR (500 MHz, CDCl 3 ): $5.36-5.26(\mathrm{~m}, 1 \mathrm{H}), 1.64(\mathrm{~s}, 9 \mathrm{H}), 1.35$ (d, J= $\left.7.0 \mathrm{~Hz}, 3 \mathrm{H}\right)$.

${ }^{13} \mathrm{C}$ NMR (125 MHz, $\left.\mathrm{CDCl}_{3}\right): 174.6,151.1,86.6,47.9,28.1,13.8$.

HRMS (ESI-TOF) m/z: [M + H]+ Calcd for $\mathrm{C}_{8} \mathrm{H}_{15} \mathrm{~N}_{2} \mathrm{O}_{5} 219.0975$ found 219.1006.

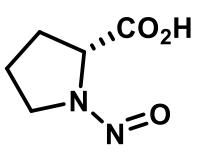

10 (cis)

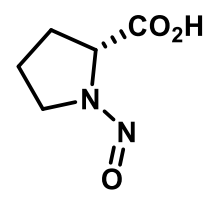

10 (trans)

nitroso-D-proline (10). General procedure A was employed using D-Proline (23 $\mathrm{mg}, 0.2 \mathrm{mmol}$ ) in dichlorethane. The reaction afforded 10 (trans) and 10 (cis) as a mixture of rotamers (28.2 $\mathrm{mg}, 98 \%$ yield) as a pale yellow oil separated by silica gel (10\% $\mathrm{MeOH}$ in DCM).

${ }^{1} \mathbf{H}$ NMR (500 MHz, $\left.\mathrm{CDCl}_{3}\right): 5.26(\mathrm{dd}, J=8.6,3.2 \mathrm{~Hz}, 1 \mathrm{H}), 4.49-4.38(\mathrm{~m}, 3 \mathrm{H}), 4.31(\mathrm{dt}, J=11.7$, $6.6 \mathrm{~Hz}, 2 \mathrm{H}), 3.64-3.55(\mathrm{~m}, 2 \mathrm{H}), 2.47-2.32(\mathrm{~m}, 3 \mathrm{H}), 2.32-2.25(\mathrm{~m}, 1 \mathrm{H}), 2.21-2.13(\mathrm{~m}, 1 \mathrm{H})$, $2.12-1.99(\mathrm{~m}, 3 \mathrm{H})$.

${ }^{13} \mathrm{C}$ NMR (125 MHz, CDCl 3$): 243.0,240.7,132.9,129.0,120.9,116.5,99.5,98.5,93.8,91.8$. 
HRMS (ESI-TOF) m/z: [M + H]+ Calcd for $\mathrm{C}_{5} \mathrm{H}_{9} \mathrm{~N}_{2} \mathrm{O}_{3} 145.0608$ found 145.0601 .

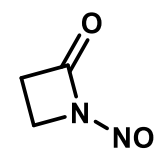

11

1-nitrosoazetidin-2-one (11). General procedure A was employed using 2-Azetidinone (16 mg, $0.2 \mathrm{mmol})$ in dicheloethane. The reaction afforded $11(18.2 \mathrm{mg}, 91 \%$ yield) as a yellow oil separated by silica gel $(5-10 \% \mathrm{MeOH}$ in DCM).

${ }^{1} \mathrm{H}$ NMR $\left(400 \mathrm{MHz}, \mathrm{CDCl}_{3}\right): 3.65(\mathrm{t}, J=5.9 \mathrm{~Hz}, 2 \mathrm{H}), 3.18(\mathrm{t}, J=5.9 \mathrm{~Hz}, 2 \mathrm{H})$.

${ }^{13} \mathrm{C} \mathrm{NMR}\left(100 \mathrm{MHz}, \mathrm{CDCl}_{3}\right): 160.8,40.7,33.9$.

HRMS (ESI-TOF) m/z: [M + H]+ Calcd for $\mathrm{C}_{3} \mathrm{H}_{5} \mathrm{~N}_{2} \mathrm{O}_{2} 101.0346$ found 101.0585 .

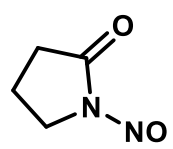

12

1-nitrosopyrrolidin-2-one (12). General procedure A was employed using 2-Pyrrolidinone (16 $\mu \mathrm{L}, 0.2 \mathrm{mmol})$ in dichloroethane. The reaction afforded $12(21.4 \mathrm{mg}, 94 \%$ yield) as a pale yellow oil separated by silica gel $(5-10 \% \mathrm{MeOH}$ in $\mathrm{DCM})$. The data matches those previously reported. ${ }^{[5]}$

${ }^{1} \mathrm{H}$ NMR $\left(500 \mathrm{MHz}, \mathrm{CDCl}_{3}\right): 3.73-3.63(\mathrm{~m}, 2 \mathrm{H}), 2.79(\mathrm{t}, J=8.1 \mathrm{~Hz}, 2 \mathrm{H}), 2.25-2.09(\mathrm{~m}, 2 \mathrm{H})$.

${ }^{13} \mathrm{C}$ NMR (125 MHz, $\left.\mathrm{CDCl}_{3}\right)$ : 173.1, 42.7, 31.0, 15.9.

HRMS (ESI-TOF) m/z: [M + H]+ Calcd for $\mathrm{C}_{4} \mathrm{H}_{7} \mathrm{~N}_{2} \mathrm{O}_{2} 115.0502$ found 115.0499 .

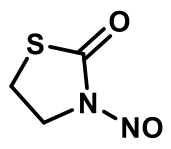

13

3-nitrosothiazolidin-2-one (13). General procedure B was employed using 1,3-thiazolidin-2-one (21 mg, $0.2 \mathrm{mmol})$. The reaction afforded 13 ( $20.6 \mathrm{mg}, 78 \%$ yield) as a pale yellow oil separated by silica gel (5-10\% MeOH in DCM).

${ }^{1} \mathrm{H}$ NMR $\left(500 \mathrm{MHz}, \mathrm{CDCl}_{3}\right): 4.00(\mathrm{t}, J=7.3 \mathrm{~Hz}, 1 \mathrm{H}), 3.39(\mathrm{t}, J=7.3 \mathrm{~Hz}, 1 \mathrm{H})$.

${ }^{13} \mathrm{C}$ NMR (125 MHz, $\left.\mathrm{CDCl}_{3}\right): 171.1,43.0,23.8$. 
HRMS (ESI-TOF) m/z: [M + H]+ Calcd for $\mathrm{C}_{10} \mathrm{H}_{12} \mathrm{NO}_{4} 133.0066$ found 133.0075 .<smiles>O=C1CCCCN1NO</smiles>

14

1-nitrosopiperidin-2-one (14). General procedure A was employed using $\delta$-valerolactam (21 $\mathrm{mg}, 0.2 \mathrm{mmol})$ in dichloroethane. The reaction afforded $14(23.8 \mathrm{mg}, 93 \%$ yield $)$ as a pale yellow oil separated by silica gel $(5-10 \% \mathrm{MeOH}$ in DCM). The data matches those previously reported. ${ }^{[5]}$

${ }^{1} \mathrm{H}$ NMR $\left(500 \mathrm{MHz}, \mathrm{CDCl}_{3}\right): 3.60-3.47(\mathrm{~m}, 1 \mathrm{H}), 2.86-2.74(\mathrm{~m}, 1 \mathrm{H}), 1.92-1.81(\mathrm{~m}, 2 \mathrm{H})$.

${ }^{13} \mathrm{C}$ NMR (125 MHz, $\left.\mathrm{CDCl}_{3}\right): 170.2,43.6,34.0,21.4,20.1$.

HRMS (ESI-TOF) m/z: [M + H]+ Calcd for $\mathrm{C}_{5} \mathrm{H}_{9} \mathrm{~N}_{2} \mathrm{O}_{2} 129.0659$ found 129.0655 .

Scheme 2 Compounds (15-36)

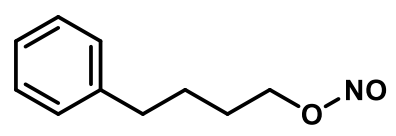

15

4-phenylbutyl nitrite (15). General procedure A was employed using 4-Phenyl-1-butanol (31 $\mu \mathrm{L}$, $0.2 \mathrm{mmol})$ in dichloroethane. The reaction afforded $15(28.6 \mathrm{mg}, 80 \%$ yield) as a pale yellow oil separated by silica gel (3-5\% ethyl acetate in hexanes). When general procedure B was employed ( $30.8 \mathrm{mg}, 86 \%$ yield). The data matches those previously reported. ${ }^{[6]}$

${ }^{1} \mathrm{H}$ NMR $\left(500 \mathrm{MHz}, \mathrm{CDCl}_{3}\right): 7.31(\mathrm{t}, J=7.3 \mathrm{~Hz}, 2 \mathrm{H}), 7.25-7.15(\mathrm{~m}, 3 \mathrm{H}), 4.73(\mathrm{~s}, 2 \mathrm{H}), 2.68(\mathrm{t}, J=$ $7.3 \mathrm{~Hz}, 2 \mathrm{H}), 1.84-1.69(\mathrm{~m}, 4 \mathrm{H})$.

${ }^{13} \mathrm{C}$ NMR (125 MHz, $\left.\mathrm{CDCl}_{3}\right): \delta 141.9,128.5,128.5,126.0,68.3,35.5,28.7,27.8$. HRMS (ESI-TOF) m/z: [M + H]+ Calcd for $\mathrm{C}_{10} \mathrm{H}_{14} \mathrm{NO}_{2} 180.1019$ found 180.1034 .

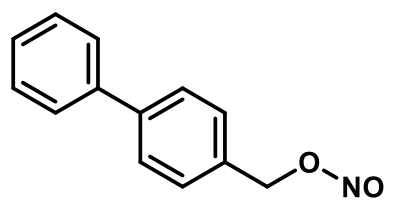

16

[1,1'-biphenyl]-4-ylmethyl nitrite (16). General procedure A was employed using Biphenyl-4methanol (37 mg, $0.2 \mathrm{mmol}$ ) in dichloroethane. The reaction afforded 16 (41.8 mg, 98\% yield) 
as a colorless solid separated by silica gel (3-5\% ethyl acetate in hexanes). When general procedure B was employed (30.9 $\mathrm{mg}, 98 \%$ yield).

${ }^{1} \mathrm{H}$ NMR (400 MHz, $\left.\mathrm{CDCl}_{3}\right): 7.61(\mathrm{t}, J=7.4 \mathrm{~Hz}, 4 \mathrm{H}), 7.41(\mathrm{qd}, J=14.9,7.3 \mathrm{~Hz}, 5 \mathrm{H}), 5.76(\mathrm{~s}, 2 \mathrm{H})$.

${ }^{13} \mathrm{C}$ NMR (100 MHz, $\left.\mathrm{CDCl}_{3}\right): 141.5,140.5,134.5,128.8,128.6,127.6,127.5,127.1,69.7$. HRMS (ESI-TOF) m/z: [M + H]+ Calcd for $\mathrm{C}_{13} \mathrm{H}_{12} \mathrm{NO}_{2} 214.0863$ found 214.0851 .<smiles>Cc1cc([N+](=O)[O-])c(CON=O)c([N+](=O)[O-])c1</smiles>

17

2,4,6-trimethylbenzyl nitrite (17). General procedure A was employed using 2,4,6Trimethylbenzyl alcohol $(30 \mathrm{mg}, 0.2 \mathrm{mmol}$ ) in dichloroethane. The reaction afforded 17 (34.8 $\mathrm{mg}, 97 \%$ yield) as a colorless solid separated by silica gel (3-5\% ethyl acetate in hexanes). When general procedure B was employed (34.6 mg, $98 \%$ yield). The data matches those previously reported. ${ }^{[7]}$

${ }^{1} \mathrm{H}$ NMR (400 MHz, $\left.\mathrm{CDCl}_{3}\right): 6.91(\mathrm{~s}, 2 \mathrm{H}), 5.72(\mathrm{~s}, 2 \mathrm{H}), 2.34(\mathrm{~s}, 6 \mathrm{H}), 2.30(\mathrm{~s}, 3 \mathrm{H})$.

${ }^{13} \mathrm{C}$ NMR (100 MHz, $\left.\mathrm{CDCl}_{3}\right):$ 138.9, 138.2, 129.3, 128.4, 64.8, 21.2, 19.7.

HRMS (ESI-TOF) m/z: [M + H]+ Calcd for $\mathrm{C}_{10} \mathrm{H}_{14} \mathrm{NO}_{2} 180.1019$ found 180.1008.

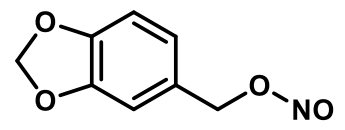

18

benzo[d][1,3]dioxol-5-ylmethyl nitrite (18). General procedure A was employed using Piperonyl alcohol (31 mg, $0.2 \mathrm{mmol}$ ) in dichloroethane. The reaction afforded $18(29.2 \mathrm{mg}, 81 \%$ yield) as a colorless oil separated by silica gel (10\% ethyl acetate in hexanes). When general procedure B was employed (34.3 $\mathrm{mg}, 95 \%$ yield).

${ }^{1} \mathrm{H}$ NMR (500 MHz, $\left.\mathrm{CDCl}_{3}\right): 6.86-6.73(\mathrm{~m}, \mathrm{~J}=7.7 \mathrm{~Hz}, 3 \mathrm{H}), 5.97(\mathrm{~s}, 2 \mathrm{H}), 5.61(\mathrm{~s}, 2 \mathrm{H})$.

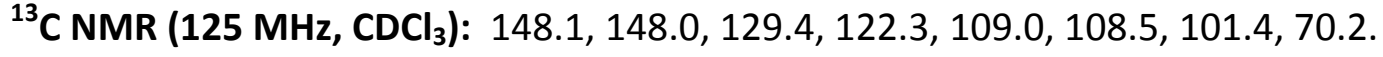

HRMS (ESI-TOF) m/z: [M + H]+ Calcd for $\mathrm{C}_{8} \mathrm{H}_{8} \mathrm{NO}_{4} 182.0448$ found 182.0415

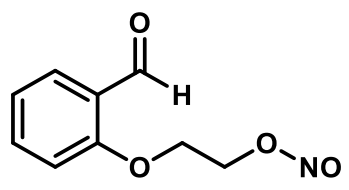


2-(2-formylphenoxy)ethyl nitrite (19). General procedure A was employed using 2-(2Hydroxyethoxy)benzaldehyde $(23 \mu \mathrm{L}, 0.2 \mathrm{mmol})$ in acetonitrile. The reaction afforded 19 (28.7 $\mathrm{mg}, 74 \%$ yield) as a yellow oil separated by silica gel (3-5\% ethyl acetate in hexanes). When general procedure B was employed ( $33.1 \mathrm{mg}, 85 \%$ yield).

${ }^{1} \mathbf{H}$ NMR (400 MHz, $\left.\mathrm{CDCl}_{3}\right): 10.42(\mathrm{~s}, 1 \mathrm{H}), 7.84(\mathrm{dd}, J=7.6,1.5 \mathrm{~Hz}, 1 \mathrm{H}), 7.59-7.52(\mathrm{~m}, 1 \mathrm{H}), 7.06$ $(\mathrm{t}, \mathrm{J}=7.5 \mathrm{~Hz}, 1 \mathrm{H}), 6.97(\mathrm{~d}, J=8.4 \mathrm{~Hz}, 1 \mathrm{H}), 5.20(\mathrm{~s}, 2 \mathrm{H}), 4.44-4.32(\mathrm{~m}, 2 \mathrm{H})$.

${ }^{13} \mathrm{C}$ NMR (100 MHz, $\left.\mathrm{CDCl}_{3}\right):$ 189.6, 160.7, 136.1, 128.7, 125.2, 121.6, 112.5, 66.8, 66.3.

HRMS (ESI-TOF) m/z: [M + H]+ Calcd for $\mathrm{C}_{9} \mathrm{H}_{10} \mathrm{NO}_{4} 196.0604$ found 196.0581 .

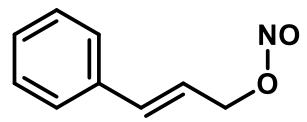

20

cinnamyl nitrite (20). General procedure A was employed using Cinnamyl alcohol (27 $\mu \mathrm{L}, 0.2$ $\mathrm{mmol})$ in dichloroethane. The reaction afforded $20(11.7 \mathrm{mg}, 36 \%$ yield) as a colorless oil separated by silica gel (3-5\% ethyl acetate in hexanes). When general procedure B was employed ( $27.5 \mathrm{mg}, 84 \%$ yield).

${ }^{1} \mathrm{H}$ NMR $\left(500 \mathrm{MHz}, \mathrm{CDCl}_{3}\right): 7.40(\mathrm{t}, J=9.3 \mathrm{~Hz}, 2 \mathrm{H}), 7.34(\mathrm{dd}, J=16.5,8.9 \mathrm{~Hz}, 2 \mathrm{H}), 7.31-7.26(\mathrm{~m}$, $1 \mathrm{H}), 6.69(\mathrm{~d}, J=15.9 \mathrm{~Hz}, 1 \mathrm{H}), 6.32(\mathrm{dt}, J=15.7,6.3 \mathrm{~Hz}, 1 \mathrm{H}), 5.35(\mathrm{~s}, 2 \mathrm{H})$.

${ }^{13} \mathrm{C}$ NMR (125 MHz, $\left.\mathrm{CDCl}_{3}\right):$ : 136.1, 134.9, 128.8, 128.4, 126.8, 122.8, 68.7.

HRMS (ESI-TOF) m/z: [M + H]+ Calcd for $\mathrm{C}_{9} \mathrm{H}_{10} \mathrm{NO}_{2} 164.0706$ found 164.0698 .

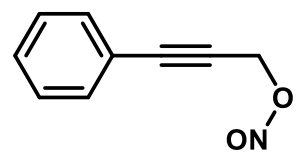

21

3-phenylprop-2-yn-1-yl nitrite (21). General procedure A was employed using 3-Phenyl-2propyn-1-ol ( $27 \mu \mathrm{L}, 0.2 \mathrm{mmol})$ in acetonitrile. The reaction afforded 21 (17.3 $\mathrm{mg}, 54 \%$ yield) as a colorless oil separated by silica gel (10\% ethyl acetate in hexanes). When general procedure B was employed (19.8 mg, 62\% yield).

${ }^{1} \mathbf{H}$ NMR (500 MHz, $\left.\mathrm{CDCl}_{3}\right): 7.47-7.44(\mathrm{~m}, 2 \mathrm{H}), 7.36-7.30(\mathrm{~m}, 3 \mathrm{H}), 5.49(\mathrm{~s}, 2 \mathrm{H})$.

${ }^{13} \mathrm{C}$ NMR (125 MHz, CDCl $)$ : 132.0, 131.8, 129.0, 128.5, 122.1, 87.0, 51.9.

HRMS (ESI-TOF) m/z: [M + H]+ Calcd for $\mathrm{C}_{9} \mathrm{H}_{8} \mathrm{NO}_{2} 162.0550$ found 162.0548 


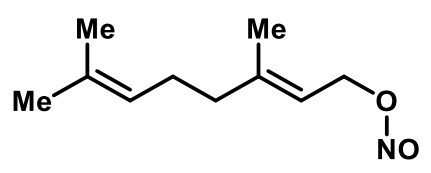

22

(E)-3,7-dimethylocta-2,6-dien-1-yl nitrite (22). General procedure A was employed using Geraniol ( $35 \mu \mathrm{L}, 0.2 \mathrm{mmol}$ ) in dichloroethane. The reaction afforded $22(32.1 \mathrm{mg}, 88 \%$ yield) as a pale yellow oil separated by silica gel (3-5\% ethyl acetate in hexanes). When general procedure B was employed ( $32.1 \mathrm{mg}, 85 \%$ yield).

${ }^{1} \mathrm{H}$ NMR (500 MHz, $\left.\mathrm{CDCl}_{3}\right): 5.37(\mathrm{t}, J=6.5 \mathrm{~Hz}, 1 \mathrm{H}), 5.21-5.02(\mathrm{~m}, J=22.4,16.7 \mathrm{~Hz}, 3), 2.17-$ $1.99(\mathrm{~m}, 4 \mathrm{H}), 1.74(\mathrm{~s}, 3 \mathrm{H}), 1.68(\mathrm{~s}, 3 \mathrm{H}), 1.60(\mathrm{~s}, 3 \mathrm{H})$.

${ }^{13} \mathrm{C}$ NMR (125 MHz, $\left.\mathrm{CDCl}_{3}\right): 143.5,132.1,123.7,117.6,64.9,39.7,26.4,25.8,17.8,16.7$.

HRMS (ESI-TOF) m/z: [M + H]+ Calcd for $\mathrm{C}_{10} \mathrm{H}_{24} \mathrm{NO}_{3} \mathrm{Si} 184.1338$ found 184.1322 .

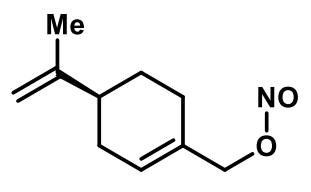

23

(S)-(4-(prop-1-en-2-yl)cyclohex-1-en-1-yl)methyl nitrite (23). General procedure A was employed using (S)-(-)-Perillyl alcohol (38 $\mu \mathrm{L}, 0.2 \mathrm{mmol}$ ) in dichloroethane. The reaction afforded 23 (30.2 mg, 84\% yield) as a pale yellow oil separated by silica gel (3-5\% ethyl acetate in hexanes). When general procedure B was employed ( $18.7 \mathrm{mg}, 52 \%$ yield).

${ }^{1} \mathrm{H}$ NMR (400 MHz, $\left.\mathrm{CDCl}_{3}\right): 5.80(\mathrm{~s}, 1 \mathrm{H}), 5.08(\mathrm{~s}, 2 \mathrm{H}), 4.72(\mathrm{~d}, \mathrm{~J}=9.1 \mathrm{~Hz}, 2 \mathrm{H}), 2.21-1.95(\mathrm{~m}, 4 \mathrm{H})$, $1.90-1.81(\mathrm{~m}, 1 \mathrm{H}), 1.74(\mathrm{~s}, 3 \mathrm{H}), 1.55-1.44(\mathrm{~m}, 1 \mathrm{H})$.

${ }^{13} \mathrm{C}$ NMR (100 MHz, $\left.\mathrm{CDCl}_{3}\right): 149.6,132.5,127.1,109.0,72.7,40.9,30.6,27.4,26.6,20.9$.

HRMS (ESI-TOF) m/z: [M + H]+ Calcd for $\mathrm{C}_{10} \mathrm{H}_{16} \mathrm{NO}_{2} 182.1176$ found 182.1161.

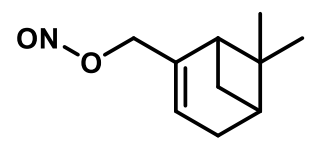

24

(6,6-dimethylbicyclo[3.1.1]hept-2-en-2-yl)methyl nitrite (24). General procedure A was employed using Myrtenol (38 $\mu \mathrm{L}, 0.2 \mathrm{mmol}$ ) in acetonitrile. The reaction afforded $24(33.3 \mathrm{mg}$, $92 \%$ yield) as a pale yellow oil separated by silica gel (3-5\% ethyl acetate in hexanes). When general procedure B was employed ( $34.3 \mathrm{mg}, 95 \%$ yield). 
${ }^{1} \mathrm{H}$ NMR $\left(500 \mathrm{MHz}, \mathrm{CDCl}_{3}\right): 5.60(\mathrm{~s}, 1 \mathrm{H}), 5.05(\mathrm{~s}, 2 \mathrm{H}), 2.41(\mathrm{dt}, J=8.7,5.6 \mathrm{~Hz}, 1 \mathrm{H}), 2.29(\mathrm{q}, J=$ $18.1 \mathrm{~Hz}, 2 \mathrm{H}), 2.11(\mathrm{~d}, J=5.2 \mathrm{~Hz}, 2 \mathrm{H}), 1.28(\mathrm{~s}, 3 \mathrm{H}), 1.18(\mathrm{~d}, J=8.7 \mathrm{~Hz}, 1 \mathrm{H}), 0.81(\mathrm{~s}, 3 \mathrm{H})$.

${ }^{13} \mathrm{C}$ NMR (125 MHz, $\left.\mathrm{CDCl}_{3}\right): 142.9,122.8,71.3,43.7,40.8,38.3,31.6,31.5,26.2,21.2$. HRMS (ESI-TOF) m/z: [M + H]+ Calcd for $\mathrm{C}_{10} \mathrm{H}_{16} \mathrm{NO}_{2} 182.1171$ found 182.1168 .

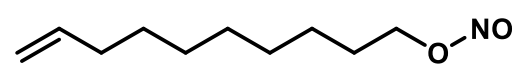

25

dec-9-en-1-yl nitrite (25). General procedure A was employed using 9-Decen-1-ol (36 $\mu \mathrm{L}, 0.2$ $\mathrm{mmol})$ in acetonitrile. The reaction afforded $25(33.9 \mathrm{mg}, 92 \%$ yield) as a pale yellow oil separated by silica gel (3-5\% ethyl acetate in hexanes). When general procedure B was employed ( $35.5 \mathrm{mg}$, $96 \%$ yield).

${ }^{1} \mathrm{H}$ NMR (500 MHz, $\left.\mathrm{CDCl}_{3}\right): 5.81$ (ddt, $\left.J=16.9,10.1,6.7 \mathrm{~Hz}, 1 \mathrm{H}\right), 4.99(\mathrm{~d}, J=17.1 \mathrm{~Hz}, 1 \mathrm{H}), 4.93(\mathrm{~d}$, $J=10.1 \mathrm{~Hz}, 1 \mathrm{H}), 4.69(\mathrm{~s}, 2 \mathrm{H}), 2.04(\mathrm{dd}, J=14.0,6.9 \mathrm{~Hz}, 2 \mathrm{H}), 1.79-1.65(\mathrm{~m}, 2 \mathrm{H}), 1.42-1.22(\mathrm{~m}$, $10 \mathrm{H})$.

${ }^{13} \mathrm{C}$ NMR (125 MHz, CDCl $)$ : 139.3, 114.3, 114.3, 68.6, 33.9, 29.5, 29.3, 29.1, 29.0, 26.0. HRMS (ESI-TOF) m/z: [M + H]+ Calcd for $\mathrm{C}_{10} \mathrm{H}_{20} \mathrm{NO}_{2} 186.1494$ found 186.1977 .

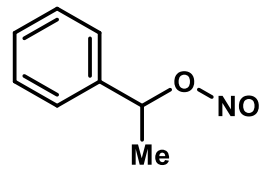

26

1-phenylethyl nitrite (26). General procedure A was employed using 1-Phenylethanol (25 $\mu \mathrm{L}$, $0.2 \mathrm{mmol})$ in dichloroethane. The reaction afforded $26(19.8 \mathrm{mg}, 66 \%$ yield) as a pale yellow oil separated by silica gel (3-5\% ethyl acetate in hexanes). When general procedure B was employed ( $20.8 \mathrm{mg}, 69 \%$ yield).

${ }^{1} \mathrm{H}$ NMR $\left(500 \mathrm{MHz}, \mathrm{CDCl}_{3}\right): 7.46-7.28(\mathrm{~m}, 5 \mathrm{H}), 6.52-6.38(\mathrm{~m}, 1 \mathrm{H}), 1.72(\mathrm{~d}, J=6.7 \mathrm{~Hz}, 3 \mathrm{H})$.

${ }^{13} \mathrm{C}$ NMR (125 MHz, $\left.\mathrm{CDCl}_{3}\right): 141.3,128.7,128.1,126.2,77.2,22.2$.

HRMS (ESI-TOF) m/z: [M + H]+ Calcd for $\mathrm{C}_{8} \mathrm{H}_{10} \mathrm{NO}_{2} 152.0712$ found 152.0699 .

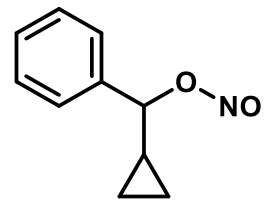

27

cyclopropyl(phenyl)methyl nitrite (27). General procedure A was employed using $\alpha$ Cyclopropylbenzyl alcohol $(29 \mu \mathrm{L}, 0.2 \mathrm{mmol}$ ) in dichloroethane. The reaction afforded 27 (16.9 
$\mathrm{mg}, 48 \%$ yield) as a pale yellow oil separated by silica gel (3-5\% ethyl acetate in hexanes). When general procedure B was employed ( $13.7 \mathrm{mg}, 39 \%$ yield).

${ }^{1}$ H NMR (400 MHz, $\left.\mathrm{CDCl}_{3}\right): 7.47-7.28(\mathrm{~m}, 5 \mathrm{H}), 5.68(\mathrm{~d}, \mathrm{~J}=8.8 \mathrm{~Hz}, 1 \mathrm{H}), 1.54-1.41(\mathrm{~m}, 1 \mathrm{H}), 0.75$ $-0.68(\mathrm{~m}, 2 \mathrm{H}), 0.52(\mathrm{dt}, J=14.1,9.2 \mathrm{~Hz}, 2 \mathrm{H})$.

${ }^{13} \mathrm{C}$ NMR (100 MHz, $\left.\mathrm{CDCl}_{3}\right):$ 140.0, 128.7, 128.3, 126.8, 85.7, 16.7, 4.5, 3.9.

HRMS (ESI-TOF) m/z: [M + H]+ Calcd for $\mathrm{C}_{10} \mathrm{H}_{12} \mathrm{NO}_{2} 178.0863$ found 178.0852 .

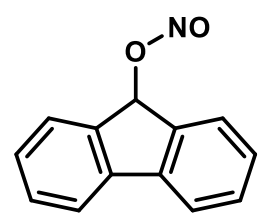

28

9H-fluoren-9-yl nitrite (28). General procedure A was employed using 9-Hydroxyfluorene (36 $\mathrm{mg}, 0.2 \mathrm{mmol})$ in dichloroethane. The reaction afforded $28(28.8 \mathrm{mg}, 68 \%$ yield) as a colorless solid separated by silica gel (3-5\% ethyl acetate in hexanes). When general procedure $B$ was employed ( $25.5 \mathrm{mg}, 60 \%$ yield).

${ }^{1} \mathrm{H}$ NMR $\left(500 \mathrm{MHz} \mathrm{CDCl}_{3}\right): 7.71(\mathrm{~d}, J=7.5 \mathrm{~Hz}, 2 \mathrm{H}), 7.48-7.42(\mathrm{~m}, 4 \mathrm{H}), 7.32(\mathrm{t}, J=7.4 \mathrm{~Hz}, 2 \mathrm{H})$, $7.27(\mathrm{~s}, 1 \mathrm{H})$.

${ }^{13}$ C NMR (125 MHz, CDCl $)$ : 141.7, 141.0, 129.9, 128.1, 125.7, 120.4, 80.3.

HRMS (ESI-TOF) m/z: [M + H]+ Calcd for $\mathrm{C}_{13} \mathrm{H}_{10} \mathrm{NO}_{2}[\mathrm{M}+\mathrm{H}]^{+} 212.0712$ found 212.0731 .

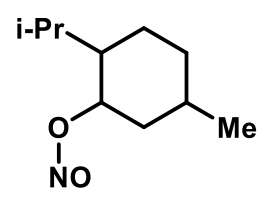

29

2-isopropyl-5-methylcyclohexyl nitrite (29). General procedure A was employed using ( \pm )menthol (31 mg, $0.2 \mathrm{mmol}$ ) in dichloroethane. The reaction afforded $\mathbf{2 9}(29.6 \mathrm{mg}, 80 \%$ yield) as a pale yellow oil separated by silica gel (3-5\% ethyl acetate in hexanes). When general procedure B was employed $\left(35.5 \mathrm{mg}, 96 \%\right.$ yield). The data matches those previously reported. ${ }^{[8]}$

${ }^{1} \mathrm{H}$ NMR (400 MHz, $\left.\mathrm{CDCl}_{3}\right): 5.37-5.14(\mathrm{~m}, 1 \mathrm{H}), 2.09-1.95(\mathrm{~m}, 1 \mathrm{H}), 1.88-1.70(\mathrm{~m}, 3 \mathrm{H}), 1.69-$ $1.53(\mathrm{~m}, 1 \mathrm{H}), 1.53-1.41(\mathrm{~m}, 1 \mathrm{H}), 1.29-1.11(\mathrm{~m}, 2 \mathrm{H}), 1.04-0.83(\mathrm{~m}, 7 \mathrm{H}), 0.77(\mathrm{~d}, \mathrm{~J}=6.9 \mathrm{~Hz}$, $3 \mathrm{H})$.

${ }^{13} \mathrm{C}$ NMR (100 MHz, $\left.\mathrm{CDCl}_{3}\right):$ 80.1, 46.7, 42.2, 34.2, 31.9, 25.9, 23.6, 22.2, 20.9, 15.9 . HRMS (ESI-TOF) m/z: [M + H]+ Calcd for $\mathrm{C}_{10} \mathrm{H}_{20} \mathrm{NO}_{2} 186.1489$ found 186.1484. 


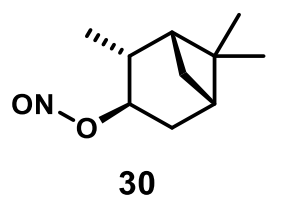

(1R,2R,3R,5S)-2,6,6-trimethylbicyclo[3.1.1] heptan-3-yl nitrite (30). General procedure A was employed using (1R,2R,3R,5S)-3-Pinanol $(31 \mathrm{mg}, 0.2 \mathrm{mmol})$ in dichloroethane. The reaction afforded $\mathbf{3 0}$ (28.4 mg, 78\% yield) as a pale yellow oil separated by silica gel (3-5\% ethyl acetate in hexanes). When general procedure B was employed ( $33.2 \mathrm{mg}, 91 \%$ yield).

${ }^{1} \mathrm{H}$ NMR $\left(500 \mathrm{MHz}, \mathrm{CDCl}_{3}\right): 5.69(\mathrm{~s}, 1 \mathrm{H}), 2.71-2.57(\mathrm{~m}, 1 \mathrm{H}), 2.46-2.36(\mathrm{~m}, 1 \mathrm{H}), 2.22-2.12(\mathrm{~m}$, $1 \mathrm{H}), 2.04-1.95(\mathrm{~m}, 1 \mathrm{H}), 1.93-1.88(\mathrm{~m}, 1 \mathrm{H}), 1.88-1.81(\mathrm{~m}, 1 \mathrm{H}), 1.27(\mathrm{~s}, 3 \mathrm{H}), 1.14(\mathrm{~d}, J=7.4 \mathrm{~Hz}$, $3 \mathrm{H}), 1.08-1.01(\mathrm{~m}, 3 \mathrm{H})$.

${ }^{13} \mathrm{C}$ NMR (125 MHz, CDCl$\left.)_{3}\right): 79.3,47.5,43.8,41.4,38.7,35.9,33.9,27.6,24.0,20.1$. HRMS (ESI-TOF) m/z: [M + H]+ Calcd for $\mathrm{C}_{10} \mathrm{H}_{18} \mathrm{NO}_{2} 184.1332$ found 184.1326 .<smiles>C=C(C)[C@H]1CC=C(C)C(O[N+](=O)[O-])C1</smiles>

31 (trans)<smiles>C=C(C)[C@H]1CC=C(C)[C@H](ON=O)C1</smiles>

31 (cis)

(1R,5S)-2-methyl-5-(prop-1-en-2-yl)cyclohex-2-en-1-yl nitrite (31 trans) and (1S,5S)-2-methyl5-(prop-1-en-2-yl)cyclohex-2-en-1-yl nitrite (31 cis). General procedure A was employed using (-)-carveol ( $32 \mu \mathrm{L}, 0.2 \mathrm{mmol}$ ) in dichloroethane. The reaction afforded $\mathbf{3 1}$ (trans) and $\mathbf{3 1}$ (cis) as a mixture of rotamers in a 1:1.25 (trans:cis) ratio $(27.1 \mathrm{mg}, 75 \%$ yield) as a pale yellow oil separated by silica gel (5-10\% ethyl acetate in hexanes). When general procedure B was employed (33.0 $\mathrm{mg}$, 91\% yield).

(31 trans) and (31 cis)

${ }^{1} \mathrm{H}$ NMR $\left(500 \mathrm{MHz}, \mathrm{CDCl}_{3}\right): 6.06-5.95(\mathrm{~m}, 1 \mathrm{H}), 5.83-5.79(\mathrm{~m}, 1 \mathrm{H}), 5.77(\mathrm{~s}, 1 \mathrm{H}), 5.73-5.65(\mathrm{~m}$, $1 \mathrm{H}), 4.78-4.69(\mathrm{~m}, 4 \mathrm{H}), 2.52-2.41(\mathrm{~m}, 1 \mathrm{H}), 2.35-2.11(\mathrm{~m}, 5 \mathrm{H}), 2.06-1.99(\mathrm{~m}, 2 \mathrm{H}), 1.95-$ $1.88(\mathrm{~m}, 2 \mathrm{H}), 1.79-1.67(\mathrm{~m}, 11 \mathrm{H}), 1.61(\mathrm{~s}, 3 \mathrm{H})$.

${ }^{13} \mathrm{C}$ NMR (125 MHz, $\left.\mathrm{CDCl}_{3}\right): 148.7,148.2,132.4,123.0,128.9,127.0,109.7,109.5,40.8,35.7$, 35.2, 35.0, 30.9, 30.9, 21.0, 20.6, 20.6, 19.3 .

HRMS (ESI-TOF) m/z: [M + H]+ Calcd for $\mathrm{C}_{10} \mathrm{H}_{16} \mathrm{NO}_{2} 182.1176$ found 182.1167 . 


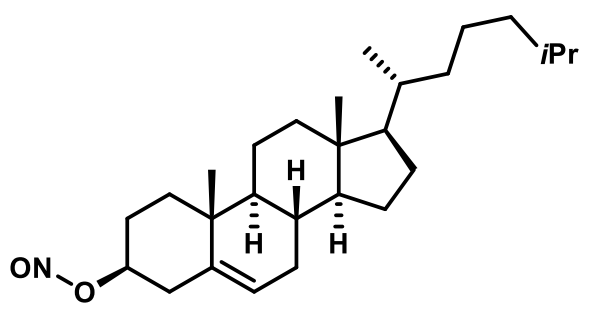

32

(3S,8S,9S,10R,13R,14S,17R)-10,13-dimethyl-17-((R)-6-methylheptan-2-yl)-

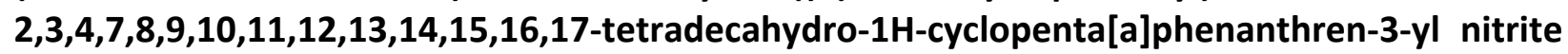
(32). General procedure A was employed using $3 \beta$-Hydroxy-5-cholestene $(83 \mathrm{mg}, 0.2 \mathrm{mmol})$ in acetonitrile. The reaction afforded $32(69.0 \mathrm{mg}, 83 \%$ yield) as a white solid separated by silica gel (20\% ethyl acetate in hexanes). When general procedure B was employed $(70.6 \mathrm{mg}, 85 \%$ yield).

${ }^{1} \mathrm{H}$ NMR (500 MHz, $\left.\mathrm{CDCl}_{3}\right): 5.49-5.42(\mathrm{~m}, 1 \mathrm{H}), 5.29-5.19(\mathrm{~m}, 1 \mathrm{H}), 2.52(\mathrm{t}, \mathrm{J}=11.6 \mathrm{~Hz}, 1 \mathrm{H})$, $2.41(\mathrm{dd}, \mathrm{J}=13.1,3.3 \mathrm{~Hz}, 1 \mathrm{H}), 2.09-1.92(\mathrm{~m}, 4 \mathrm{H}), 1.90-1.68(\mathrm{~m}, 3 \mathrm{H}), 1.65-1.45(\mathrm{~m}, 6 \mathrm{H}), 1.43$ $-1.24(\mathrm{~m}, 6 \mathrm{H}), 1.23-0.98(\mathrm{~m}, 12 \mathrm{H}), 0.93(\mathrm{~d}, \mathrm{~J}=6.5 \mathrm{~Hz}, 3 \mathrm{H}), 0.88(\mathrm{dd}, \mathrm{J}=6.5,1.9 \mathrm{~Hz}, 6 \mathrm{H}), 0.70$ $(\mathrm{s}, 3 \mathrm{H})$.

${ }^{13} \mathrm{C}$ NMR (125 MHz, $\mathrm{CDCl}_{3}$ ): 139.6, 123.2, 79.6, 56.9, 56.3, 50.3, 42.5, 39.9, 39.7, 38.9, 37.3, $36.8,36.4,36.0,32.1,32.0,28.5,28.4,28.2$, 24.5, 24.0, 23.0, 22.7, 21.2, 19.5, 18.9, 12.0 .

HRMS (ESI-TOF) m/z: [M + H]+ Calcd for $\mathrm{C}_{27} \mathrm{H}_{48} \mathrm{NO}_{2} 416.3523$ found 416.3560 .

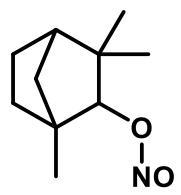

33

1,3,3-trimethylbicyclo[2.2.1]heptan-2-yl nitrite (33). General procedure B was employed using Fenchyl alcohol (32 mg, $0.2 \mathrm{mmol})$. The reaction afforded $33(19.4 \mathrm{mg}, 53 \%$ yield) as a pale yellow oil separated by silica gel (3-5\% ethyl acetate in hexanes).

${ }^{1} \mathbf{H}$ NMR (500 MHz, $\left.\mathrm{CDCl}_{3}\right): 4.93(\mathrm{~s}, 1 \mathrm{H}), 1.81(\mathrm{~d}, \mathrm{~J}=3.0 \mathrm{~Hz}, 1 \mathrm{H}), 1.70(\mathrm{~d}, \mathrm{~J}=10.8 \mathrm{~Hz}, 2 \mathrm{H}), 1.65-$ $1.57(\mathrm{~m}, 1 \mathrm{H}), 1.53-1.44(\mathrm{~m}, 1 \mathrm{H}), 1.29(\mathrm{~d}, \mathrm{~J}=10.6 \mathrm{~Hz}, 1 \mathrm{H}), 1.13(\mathrm{~s}, 3 \mathrm{H}), 1.06(\mathrm{~s}, 3 \mathrm{H}), 0.73(\mathrm{~s}, 3 \mathrm{H})$. ${ }^{13} \mathrm{C}$ NMR (125 MHz, CDCl$)$ ): 91.3, 48.7, 48.3, 41.8, 40.3, 30.3, 26.7, 26.0, 21.2, 19.2.

HRMS (ESI-TOF) m/z: [M + H]+ Calcd for $\mathrm{C}_{10} \mathrm{H}_{18} \mathrm{NO}_{2} 184.1332$ found 184.1326.

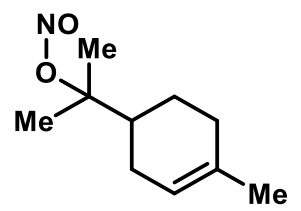


(R)-2-(4-methylcyclohex-3-en-1-yl)propan-2-yl nitrite (34). General procedure B was employed using $\alpha$-Terpineol ( $33 \mu \mathrm{L}, 0.2 \mathrm{mmol})$. The reaction afforded $34(23.0 \mathrm{mg}, 63 \%$ yield) as a teal oil separated by silica gel (3-5\% ethyl acetate in hexanes). *34 consistently appeared to decompose into an unknown compound during characterization. Tabulated data reflects desired product.

${ }^{1} \mathrm{H}$ NMR (500 MHz, $\left.\mathrm{CDCl}_{3}\right): 5.39(\mathrm{~s}, 1), 2.10-1.75(\mathrm{~m}, 5 \mathrm{H}), 1.66(\mathrm{~s}, 3 \mathrm{H}), 1.57(\mathrm{~d}, \mathrm{~J}=5.9 \mathrm{~Hz}, 6 \mathrm{H})$.

${ }^{13} \mathrm{C}$ NMR (125 MHz, $\left.\mathrm{CDCl}_{3}\right):$ 134.3, 120.2, 87.2, 44.0, 30.9, 26.7, 25.4, 24.4, 24.0, 23.4. HRMS (ESI-TOF) m/z: [M + H]+ Calcd for $\mathrm{C}_{10} \mathrm{H}_{18} \mathrm{NO}_{2} 184.1332$ found 184.1317.

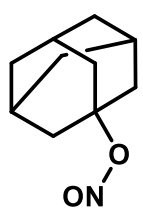

35

adamantan-1-yl nitrite (35). General procedure A was employed using 1-Adamantanol (31 mg, $0.2 \mathrm{mmol})$ in acetonitrile. The reaction afforded 35 (19.2 $\mathrm{mg}, 53 \%$ yield) as a colorless solid separated by silica gel (3-5\% ethyl acetate in hexanes). When general procedure B was employed ( $22.8 \mathrm{mg}, 63 \%$ yield).

${ }^{1} \mathrm{H}$ NMR (500 MHz, $\left.\mathrm{CDCl}_{3}\right): 2.29(\mathrm{~s}, 3 \mathrm{H}), 2.13(\mathrm{~s}, 6 \mathrm{H}), 1.76(\mathrm{~s}, 6 \mathrm{H})$.

${ }^{13} \mathrm{C}$ NMR (125 MHz, $\left.\mathrm{CDCl}_{3}\right)$ : 82.3, 42.9, 36.2, 30.9.

HRMS (ESI-TOF) m/z: [M + H]+ Calcd for $\mathrm{C}_{10} \mathrm{H}_{16} \mathrm{NO}_{2} 182.1176$ found 182.1168.

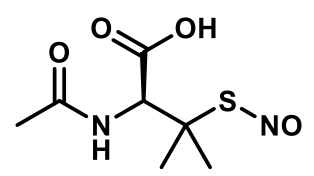

36

S-nitroso-N-acetylpenicillamine (36). General procedure B was employed using N-Acetyl-Dpenicillamine (38 mg, $0.2 \mathrm{mmol}$ ). The reaction afforded 36 ( $31.7 \mathrm{mg}, 72 \%$ yield) as a blue solid separated by silica gel (100\% ethyl acetate). The data matches those previously reported. ${ }^{[9]}$

${ }^{1}$ H NMR (500 MHz, (CD $\left.)_{2} \mathrm{SO}\right): 13.17$ (bs, $\left.1 \mathrm{H}\right), 8.51$ (d, J = 9.5 Hz, 1H), 5.17 (d, J = $\left.9.5 \mathrm{~Hz}, 1 \mathrm{H}\right)$, 3.39 (bs, 1H), 1.97 (s, 3H), $1.94(\mathrm{~s}, 3 \mathrm{H}), 1.87$ (s, 3H).

${ }^{13} \mathrm{C}$ NMR (125 MHz, $\left.\left(\mathrm{CD}_{3}\right)_{2} \mathrm{SO}\right):$ 170.9, 169.6, 59.2, 58.4, 26.3, 25.3, 22.2.

HRMS (ESI-TOF) m/z: [M + H]+ Calcd for $\mathrm{C}_{7} \mathrm{H}_{13} \mathrm{~N}_{2} \mathrm{O}_{4} \mathrm{~S} 221.0591$ found 221.0585 .

Procedure for synthesis of ${ }^{15} \mathrm{~N}$-labeled NO-1. 


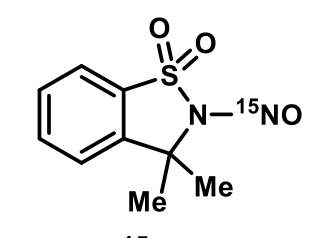

(NO-1) $-{ }^{15} \mathrm{~N}$ Labeled

3,3-dimethyl-2-[nitroso- ${ }^{15} \mathrm{~N}$ ]-2,3-dihydrobenzo[d]isothiazole 1,1-dioxide (NO-1). To a large $1 \mathrm{~L}$ round bottom $986.3 \mathrm{mg}$ ( $5 \mathrm{mmol}$ ) of DMBS was added with $10 \mathrm{~mL}$ of DCM and $1.3 \mathrm{~g}(7.0 \mathrm{mmol})$ of $p \mathrm{TsOH}$. The mixture was stirred and cooled to $0{ }^{\circ} \mathrm{C}$. Slowly added was $500.0 \mathrm{mg}(7.14 \mathrm{mmol})$ $\mathrm{NaNO}_{2}$. The reaction was left to warm to room temperature and stirred overnight. The was filtered through a Büchner funnel to remove the insoluble material. The filtrate was then absorbed onto silica and purified with column chromatography using DCM (Rf $=0.4$ ) or $10 \%$ EtOAc in hexanes as the mobile phase. Isolated was $886.2 \mathrm{mg}$ (78\% yield) of greenish yellow solid.

${ }^{1}$ H NMR (500 MHz, CDCl $): 7.89-7.77(\mathrm{~m}, 2 \mathrm{H}), 7.64(\mathrm{t}, J=7.6 \mathrm{~Hz}, 1 \mathrm{H}), 7.53(\mathrm{~d}, J=7.9 \mathrm{~Hz}, 1 \mathrm{H})$, $2.00(\mathrm{~s}, 6 \mathrm{H})$.

${ }^{13} \mathrm{C}$ NMR (125 MHz, $\left.\mathrm{CDCl}_{3}\right):$ 139.6, 135.4, 131.6, 130.3, 123.4, 122.3, 65.5, 29.8. HRMS (ESI-TOF) m/z: [M + H]+ Calcd for $\mathrm{C}_{9} \mathrm{H}_{11} \mathrm{~N}^{15} \mathrm{NO}_{3} \mathrm{~S} 228.0455$ found 228.0446 .

Scheme 3 Compounds (37-39)

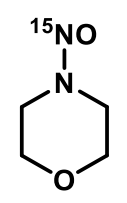

37

4-nitrosomorpholine (37). General procedure A was employed using morpholine (17 mg, 0.2 $\mathrm{mmol})$ in dichloromethane. The reaction afforded $37(22.9 \mathrm{mg}, 98 \%$ yield) as a pale yellow oil separated by silica gel (30\% ethyl acetate in hexanes).

${ }^{1} \mathrm{H}$ NMR (500 MHz, $\left.\mathrm{CDCl}_{3}\right): 4.32-4.24(\mathrm{~m}, 1 \mathrm{H}), 3.90-3.86(\mathrm{~m}, 1 \mathrm{H}), 3.86-3.82(\mathrm{~m}, 1 \mathrm{H}), 3.68-$ $3.61(\mathrm{~m}, 1 \mathrm{H})$.

${ }^{13} \mathrm{C}$ NMR (125 MHz, CDCl 3 ): 67.4 (s), 66.0 (s), 50.0 (d, $J=6.0 \mathrm{~Hz}$ ), 40.4 (d, $\left.J=1.8 \mathrm{~Hz}\right)$. HRMS (ESI-TOF) m/z: [M + H]+ Calcd for $\mathrm{C}_{4} \mathrm{H}_{9} \mathrm{~N}^{15} \mathrm{NO}_{2} 118.0629$ found 118.0631 .<smiles>CCCC1CCC(C)CC1O[Na]</smiles> 
${ }^{15} \mathrm{~N}$-labeled 2-isopropyl-5-methylcyclohexyl nitrite (38). General procedure A was employed using ( \pm )-menthol ( $31 \mathrm{mg}, 0.2 \mathrm{mmol}$ ) in dichloroethane. The reaction afforded 38 (29.8 $\mathrm{mg}, 81 \%$ yield) as a pale yellow oil separated by silica gel (3-5\% ethyl acetate in hexanes).

${ }^{1} \mathrm{H}$ NMR $\left(500 \mathrm{MHz}, \mathrm{CDCl}_{3}\right): 5.36-5.16(\mathrm{~m}, 1 \mathrm{H}), 2.02(\mathrm{~d}, \mathrm{~J}=11.6 \mathrm{~Hz}, 1 \mathrm{H}), 1.90-1.69(\mathrm{~m}, 3 \mathrm{H})$, $1.69-1.54(\mathrm{~m}, 1 \mathrm{H}), 1.48(\mathrm{t}, \mathrm{J}=11.6 \mathrm{~Hz}, 1 \mathrm{H}), 1.28-1.14(\mathrm{~m}, 2 \mathrm{H}), 1.02-0.84(\mathrm{~m}, 7 \mathrm{H}), 0.77(\mathrm{~d}, \mathrm{~J}=$ $6.8 \mathrm{~Hz}, 3 \mathrm{H})$.

${ }^{13} \mathrm{C}$ NMR (125 MHz, $\left.\mathrm{CDCl}_{3}\right): 80.1,46.7,42.2,34.2,31.9,25.9,23.6,22.2,20.9,15.9$. HRMS (ESI-TOF) m/z: [M + H]+ Calcd for $\mathrm{C}_{10} \mathrm{H}_{20}{ }^{15} \mathrm{NO}_{2} 187.1459$ found 187.1451.

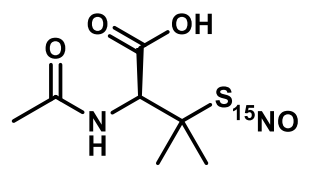

39

S-[nitroso $-{ }^{15} \mathrm{~N}$ - $\mathrm{N}$-acetylpenicillamine (39). General procedure B was employed using N-AcetylD-penicillamine (38 mg, $0.2 \mathrm{mmol})$. The reaction afforded 39 ( $31.7 \mathrm{mg}, 72 \%$ yield) as a blue solid separated by silica gel ( $100 \%$ ethyl acetate).

${ }^{1} \mathrm{H}$ NMR (500 MHz, DMSO): 13.10 (s, 1H), 8.50 (d, J = 9.5 Hz, 1H), 5.16 (d, J = 9.5 Hz, 1H), $1.97(\mathrm{~s}$, $3 \mathrm{H}), 1.94(\mathrm{~s}, 3 \mathrm{H}), 1.87(\mathrm{~s}, 3 \mathrm{H})$.

${ }^{13}$ C NMR (125 MHz, DMSO): 170.8, 170.0, 59.1, 58.3, 26.2, 25.2, 22.2.

HRMS (ESI-TOF) m/z: [M + H]+ Calcd for $\mathrm{C}_{7} \mathrm{H}_{13} \mathrm{~N}^{15} \mathrm{NO}_{4} \mathrm{~S} 222.0561$ found 222.0563 . 


\section{References}

[1] Helberg, J.; Ampßler, T.; Zipse, H. Pyridinyl Amide Ion Pairs As Lewis Base Organocatalysts. J. Org. Chem. 2020, 85, 5390-5402.

[2] Porter, N. A.; Carter, R. L.; Mero, C. L.; Roepel, M. G.; Curran, D. P. Penultimate Group Effects in Free Radical Telomerizations of Acrylamides. Tetrahedron 1996, 52, 4181-4198.

[3] Zhang, J.; Jiang, J.; Li, Y.; Wan, X. lodide-Catalyzed Synthesis of N-Nitrosamines via C-N Cleavage of Nitromethane. J. Org. Chem 2013, 78, 11366-11372.

[4] Cochrane, S. A.; Li, X.; He, S.; Yu, M.; Wu, M.; Vederas, J. C. Synthesis of TridecaptinAntibiotic Conjugates with in Vivo Activity against Gram-Negative Bacteria. Journal of Medicinal Chemistry 2015, 58 (24), 9779-9785.

[5] Torra, N.; Urpí, F.; Vilarrasa, J. N-Nitrosation and N-Nitration of Lactams. From Macrolactams to Macrolactones. Tetrahedron 1989, 45, 863-868.

[6] Doyle, M. P.; Zuidema, L. J.; Bade, T. R. Cyclic Ether Formation in Oxidations of Primary Alcohols by Cerium(IV). Reactions of 5-Phenyl-1-Pentanol, 4-Phenyl-1-Butanol, and 3Phenyl-1-Propanol with Ceric Ammonium Nitrate. J. Org. Chem. 1975, 40, 1454-1456.

[7] Suzuki, H.; Nakano, K.; Mishina, T.; Hanafusa, T. Nip. Kag. Kai. 1978, 7, 1049-1052.

[8] Holan, M.; Jahn, U. Anaerobic Nitroxide-Catalyzed Oxidation of Alcohols Using the NO+/NO. Redox Pair. Org. Lett. 2013, 16, 58-61.

[9] Roy, B.; D'hardemare, A. D. M.; Fontecave, M. New Thionitrites: Synthesis, Stability, and Nitric Oxide Generation. J. Org. Chem 1994, 59, 7019-7026. 


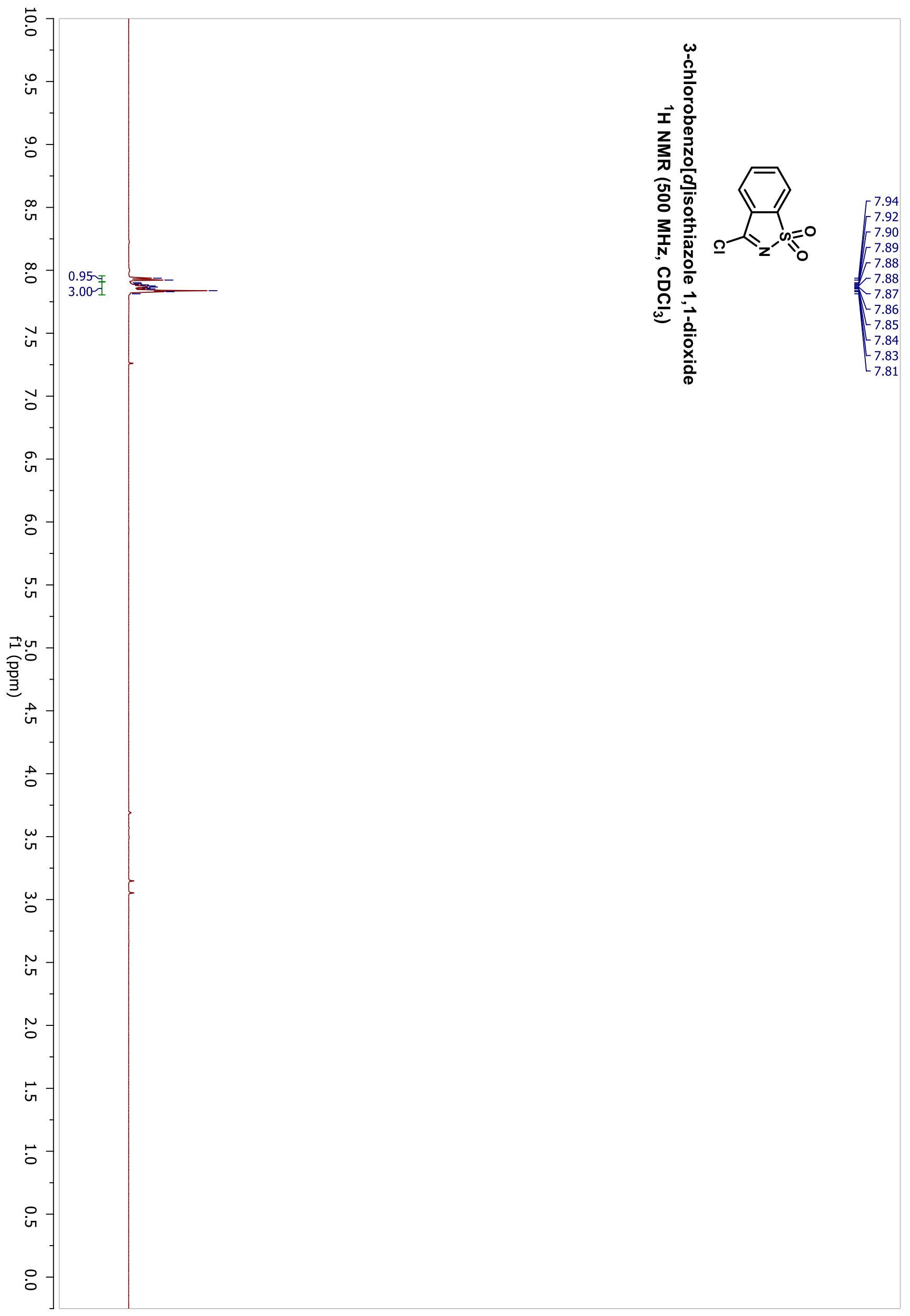




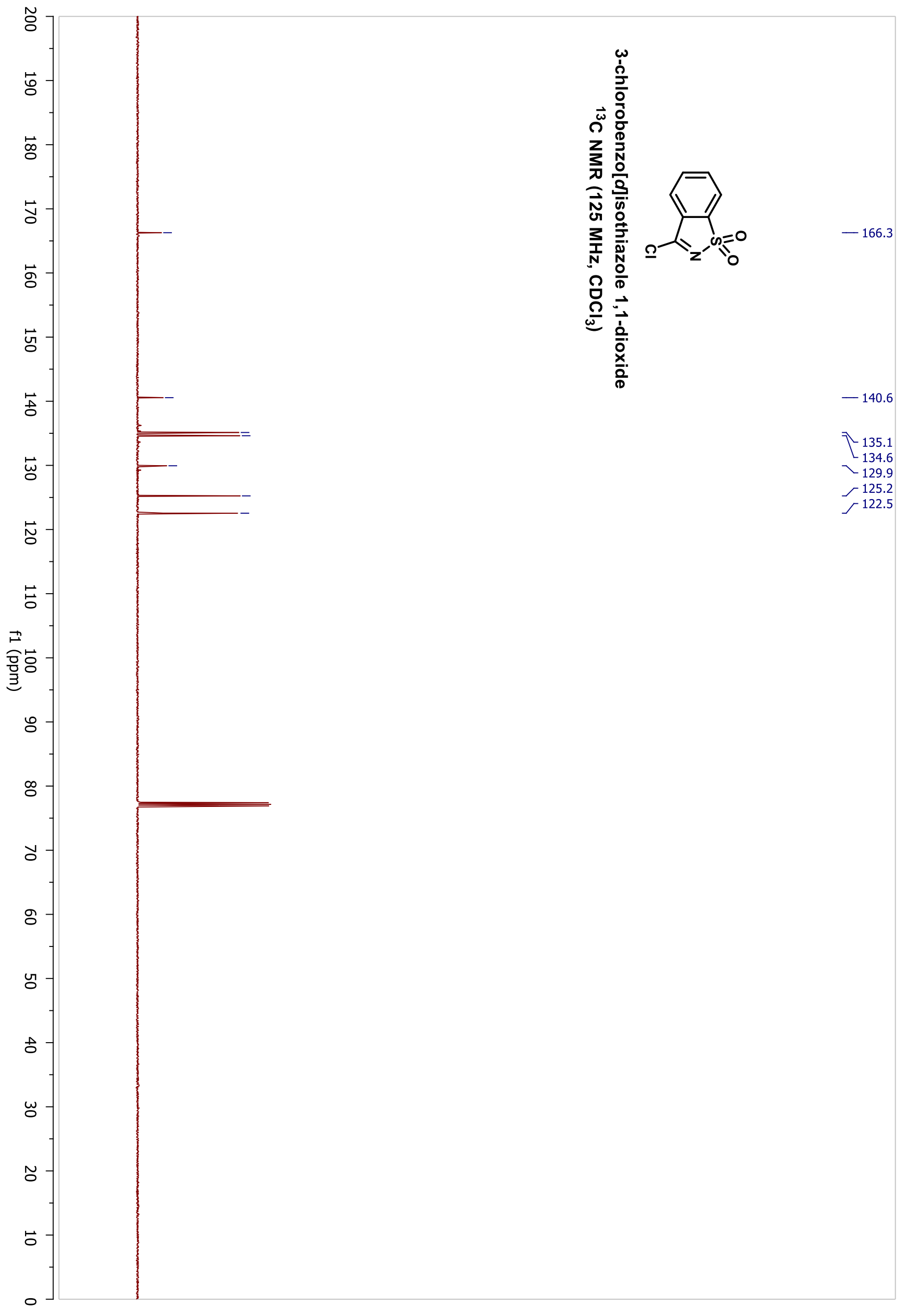



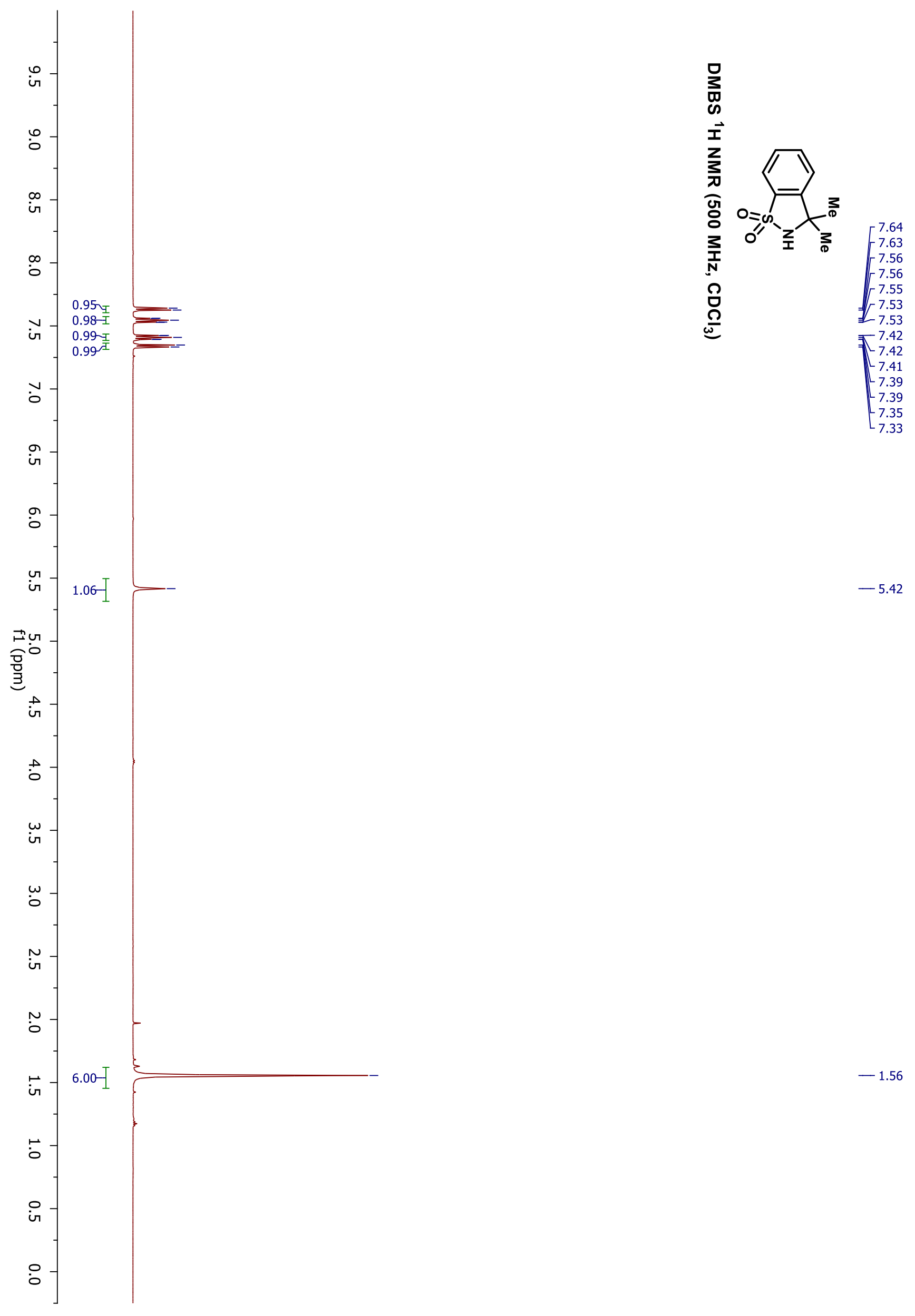

$-5.42$ 

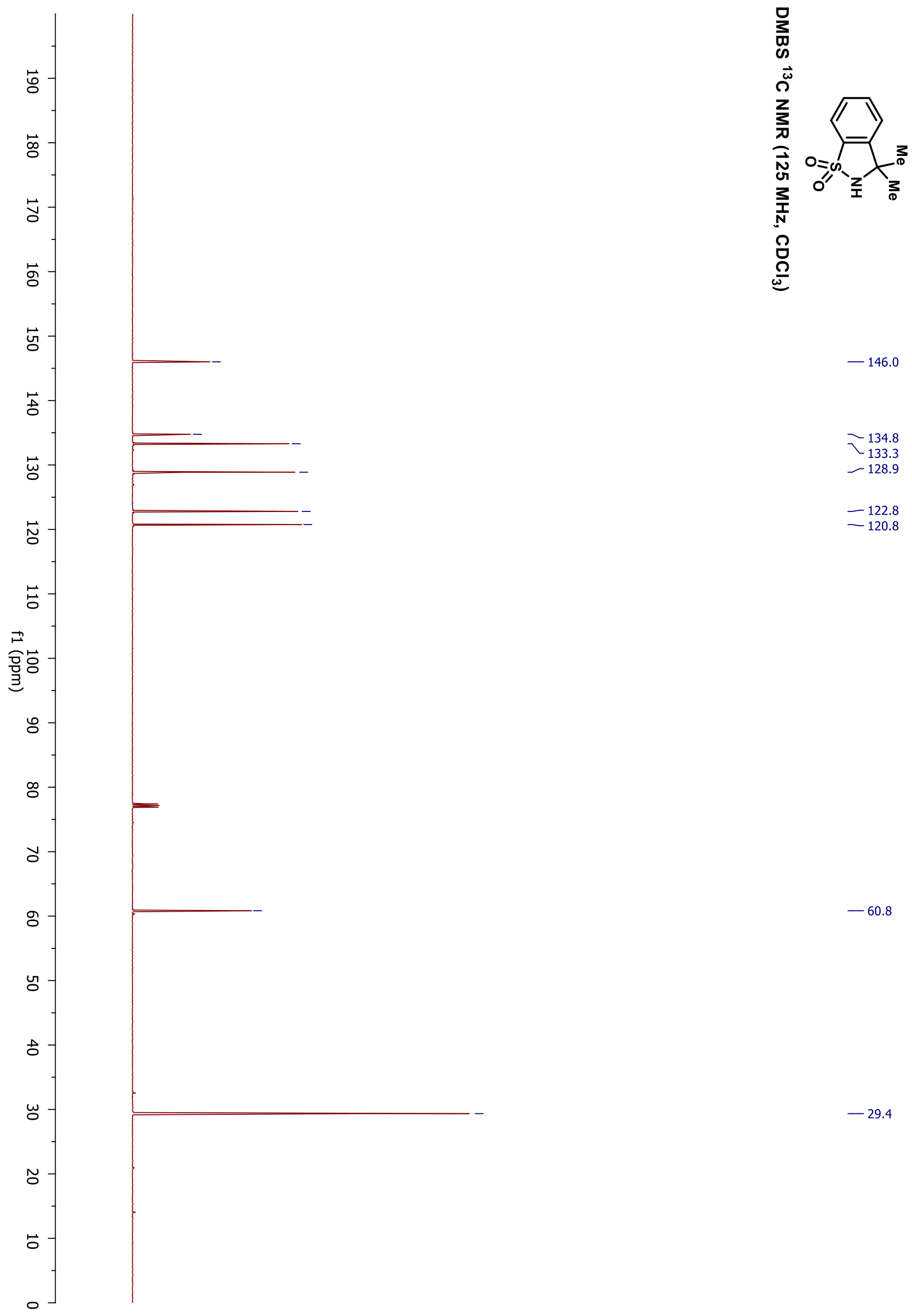

$-134.8$

133.3

$-128.9$

$-122.8$

$-120.8$

$-60.8$

$-29.4$ 

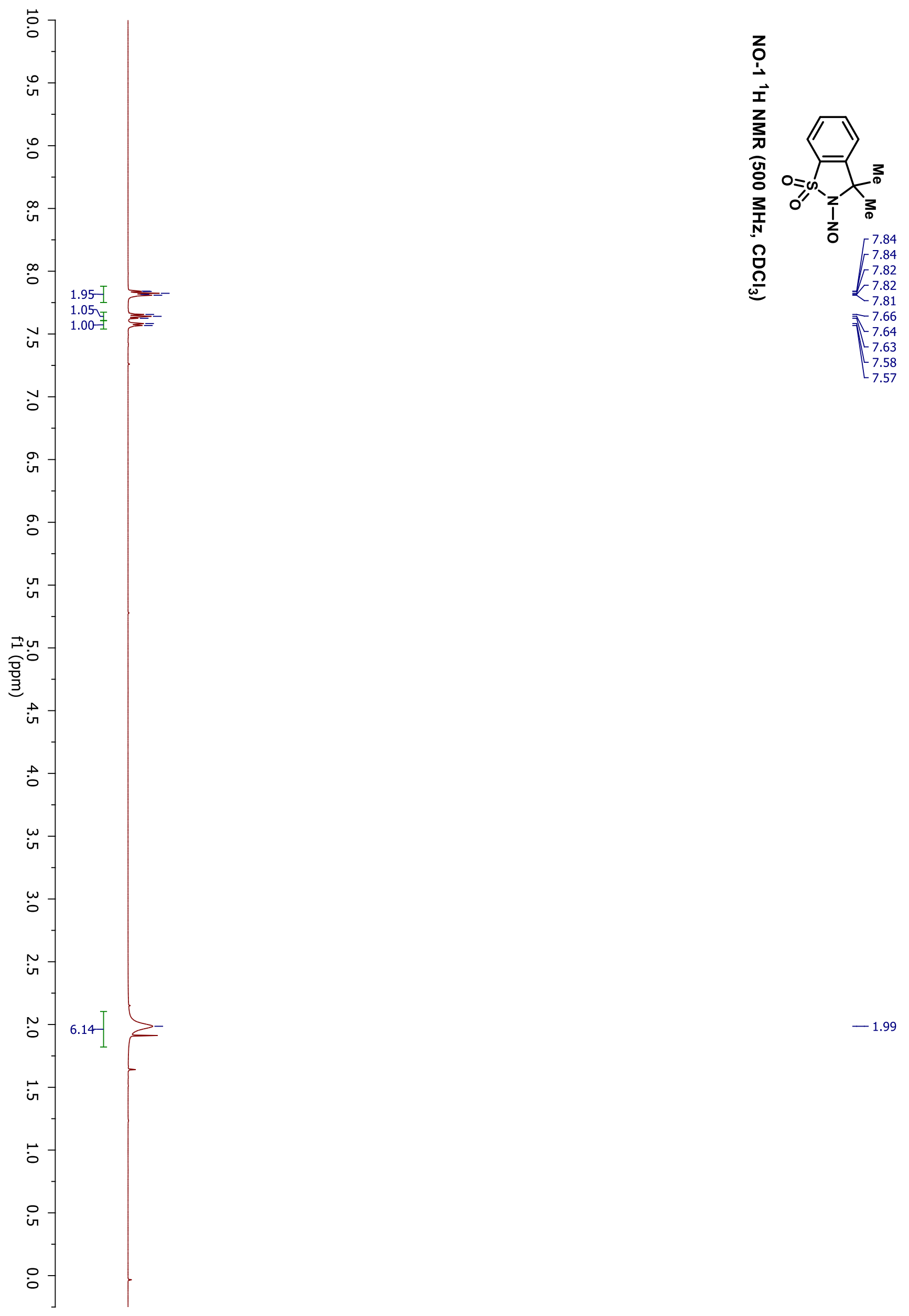

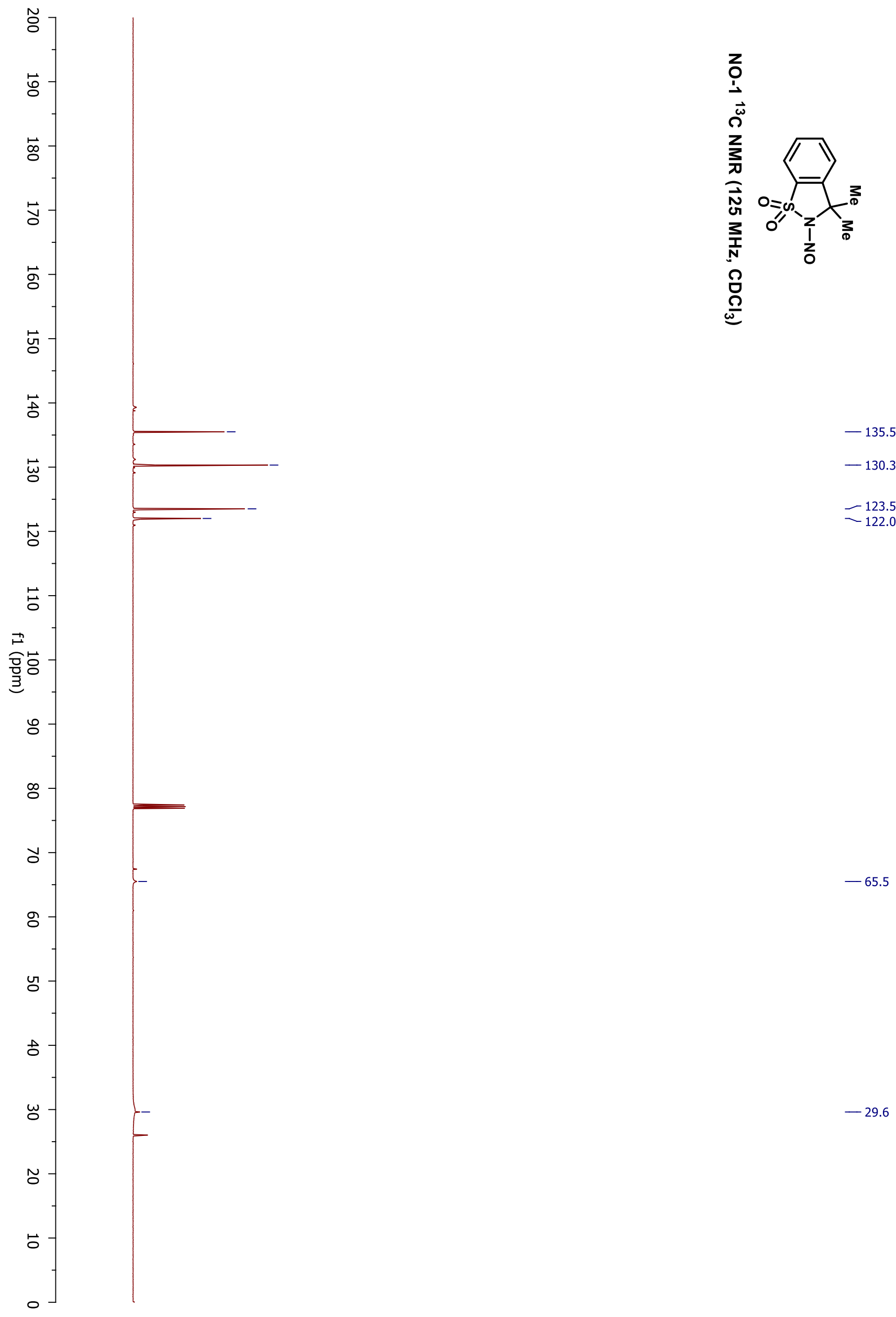

$-130.3$

-123.5
-122.0

$-65.5$

$-29.6$ 


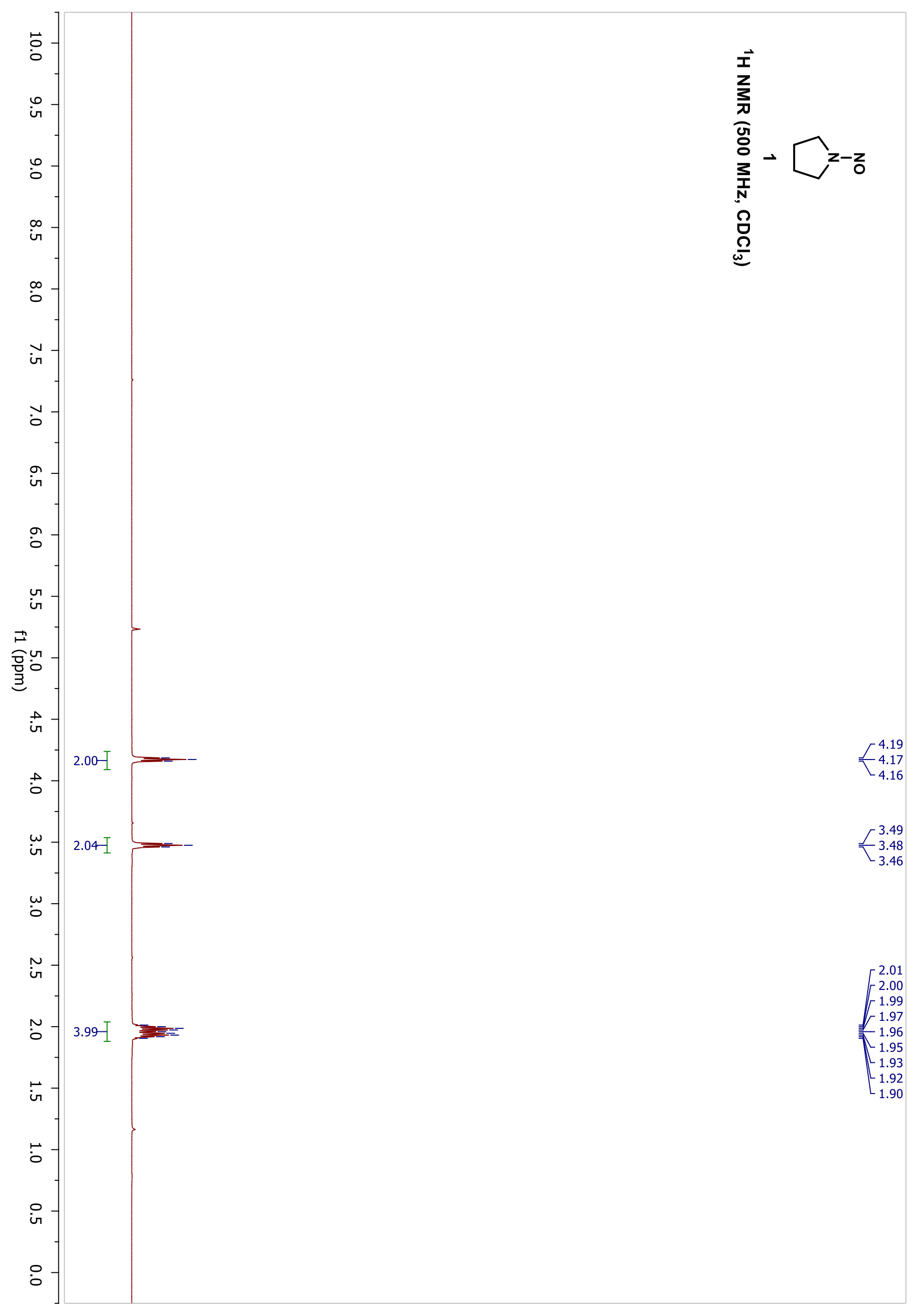




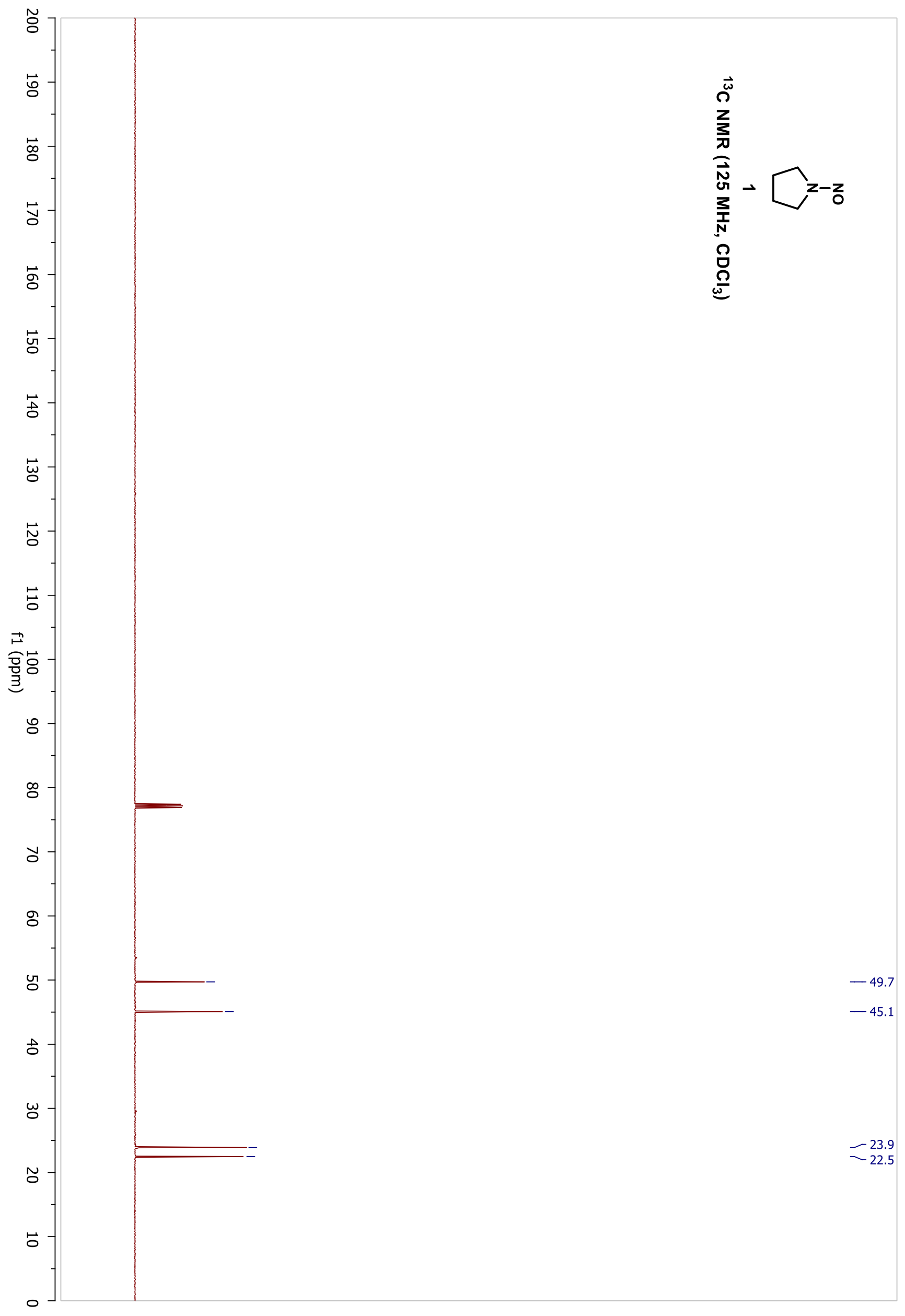




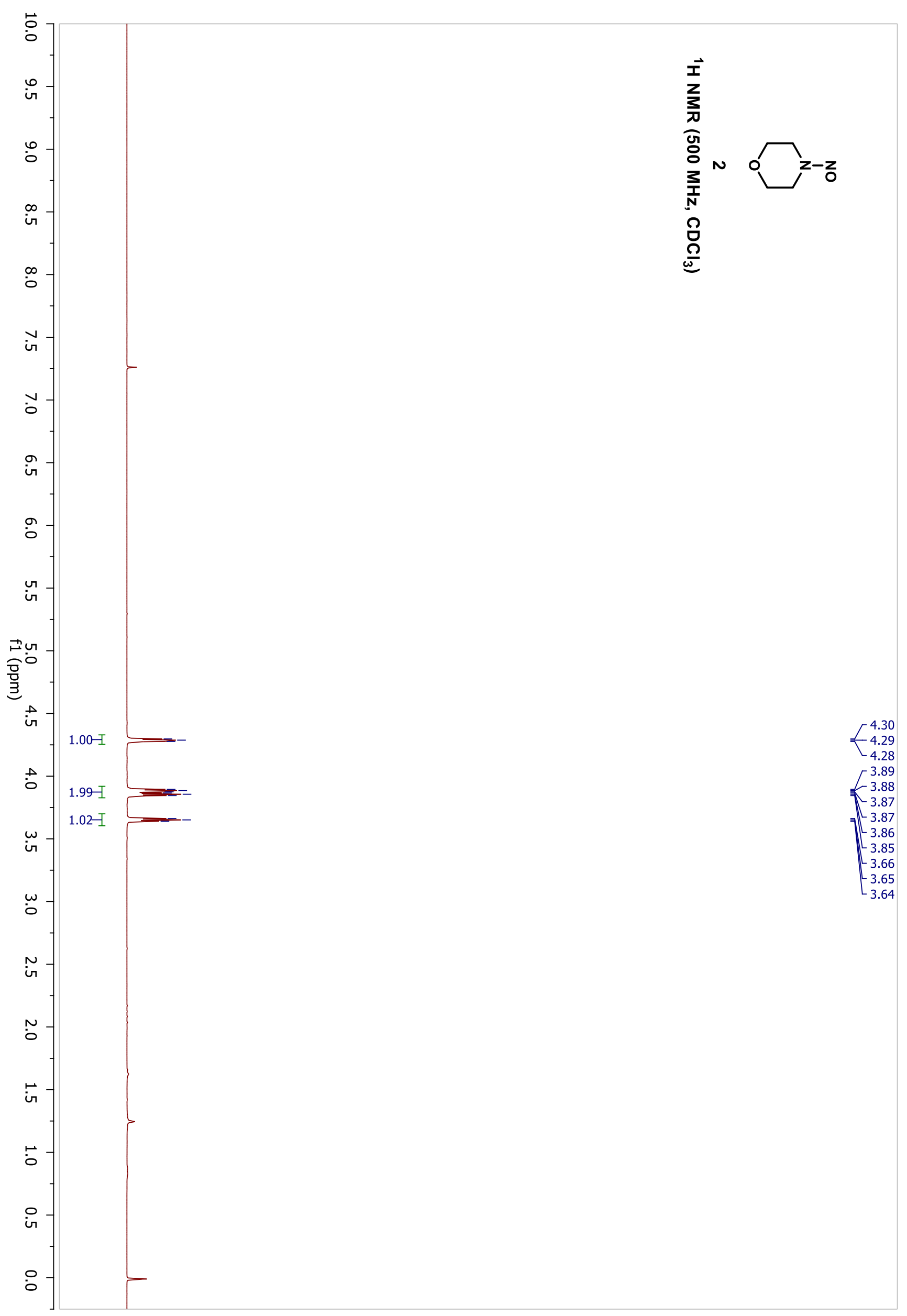




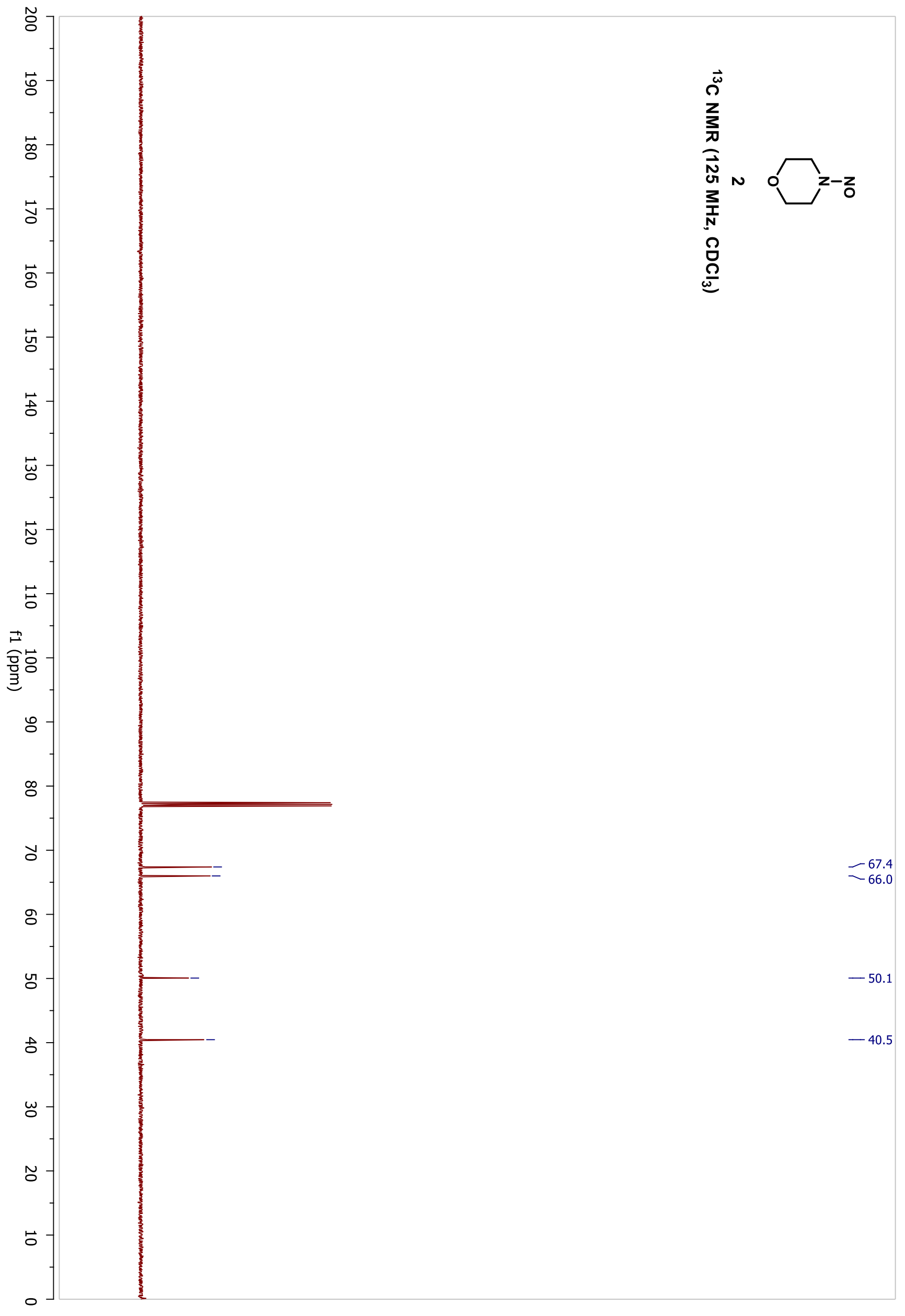




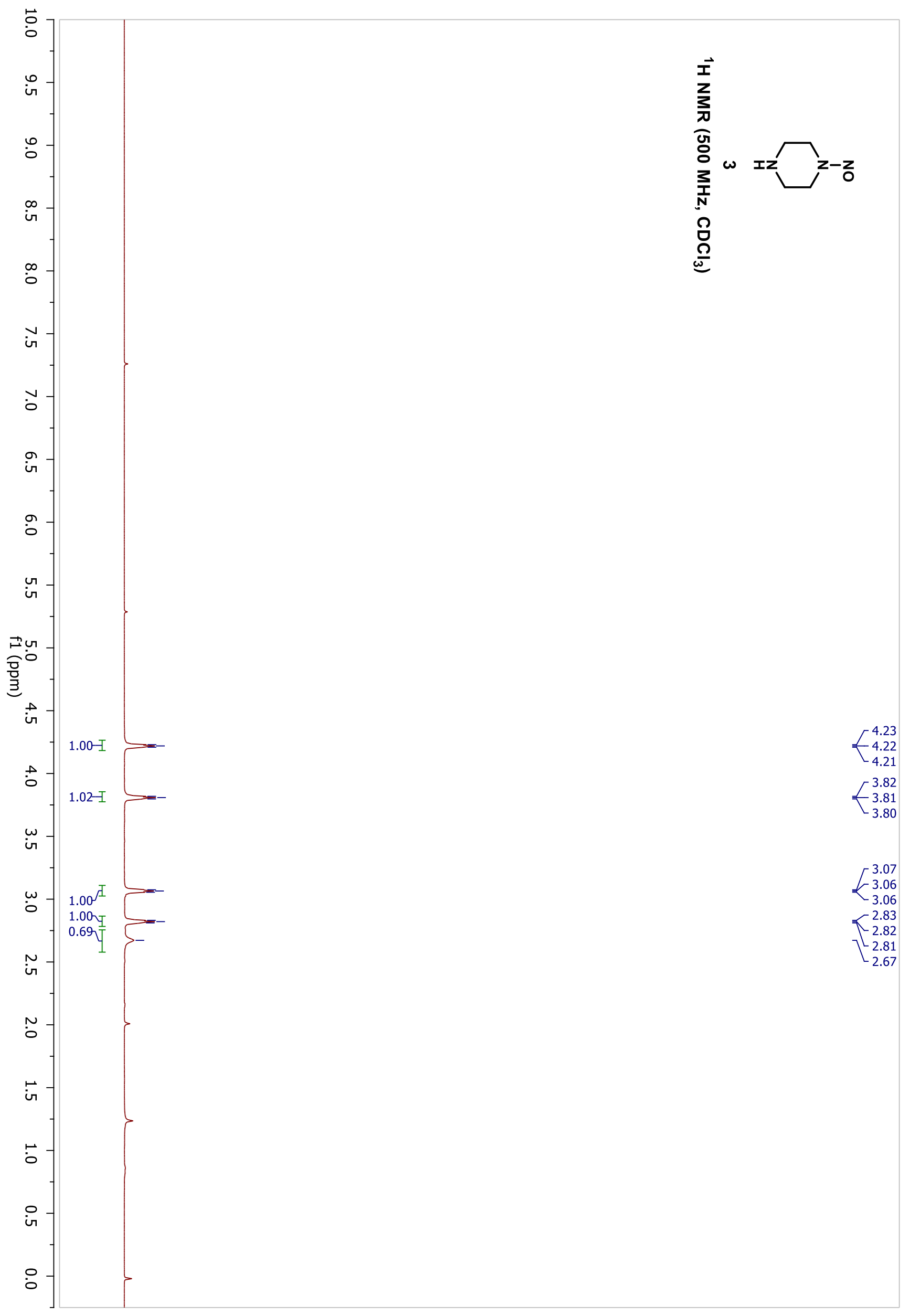




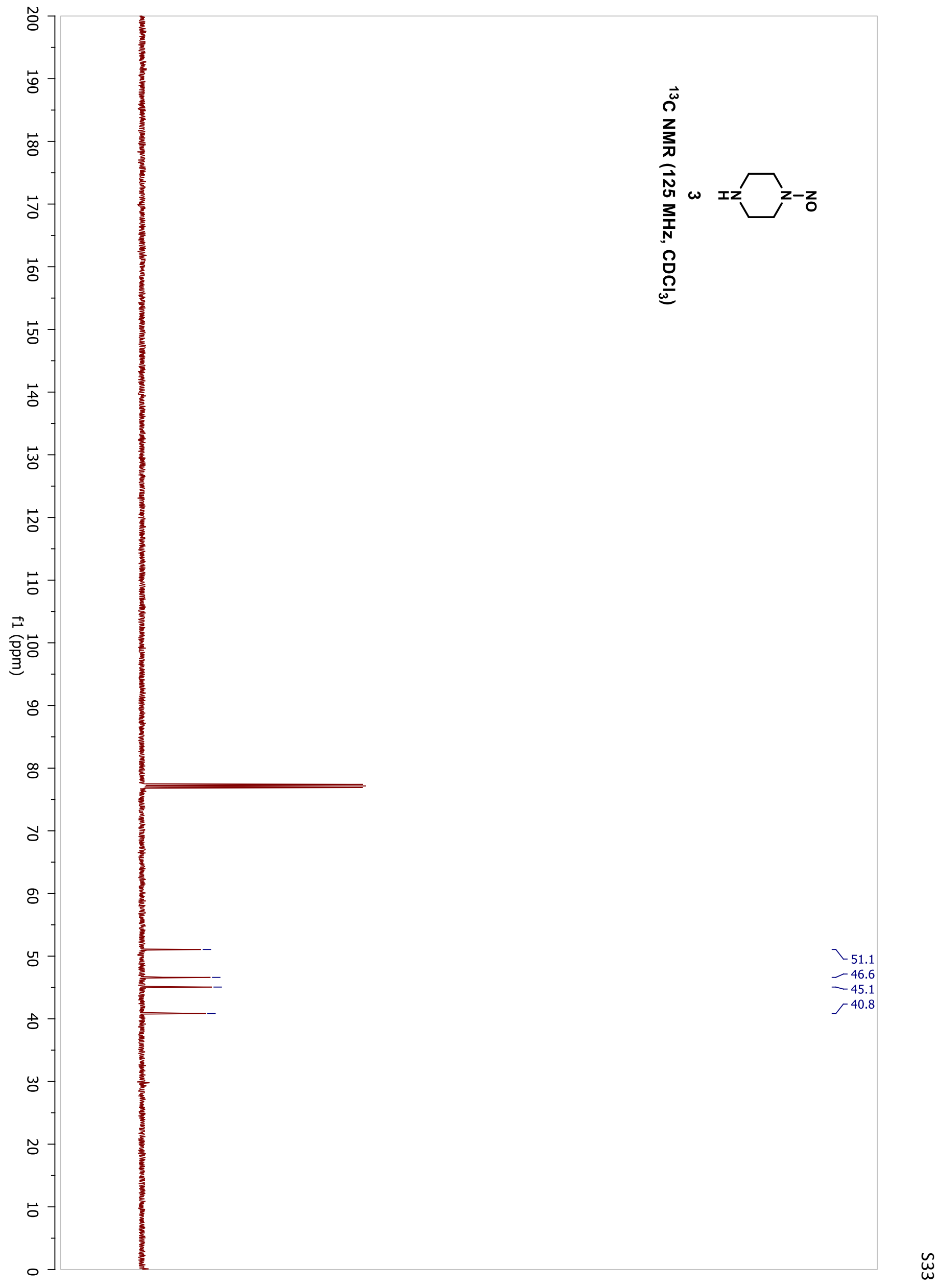




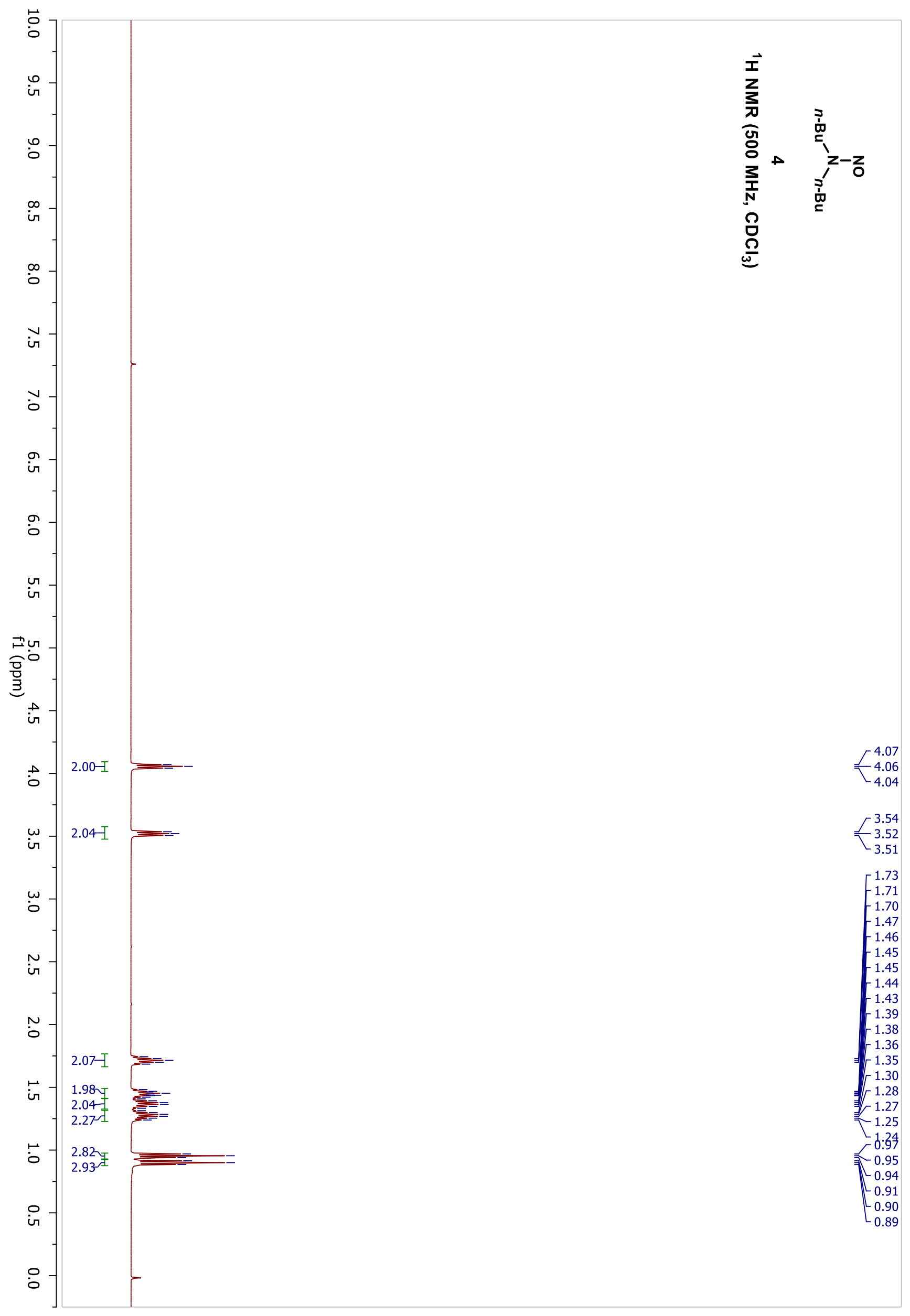




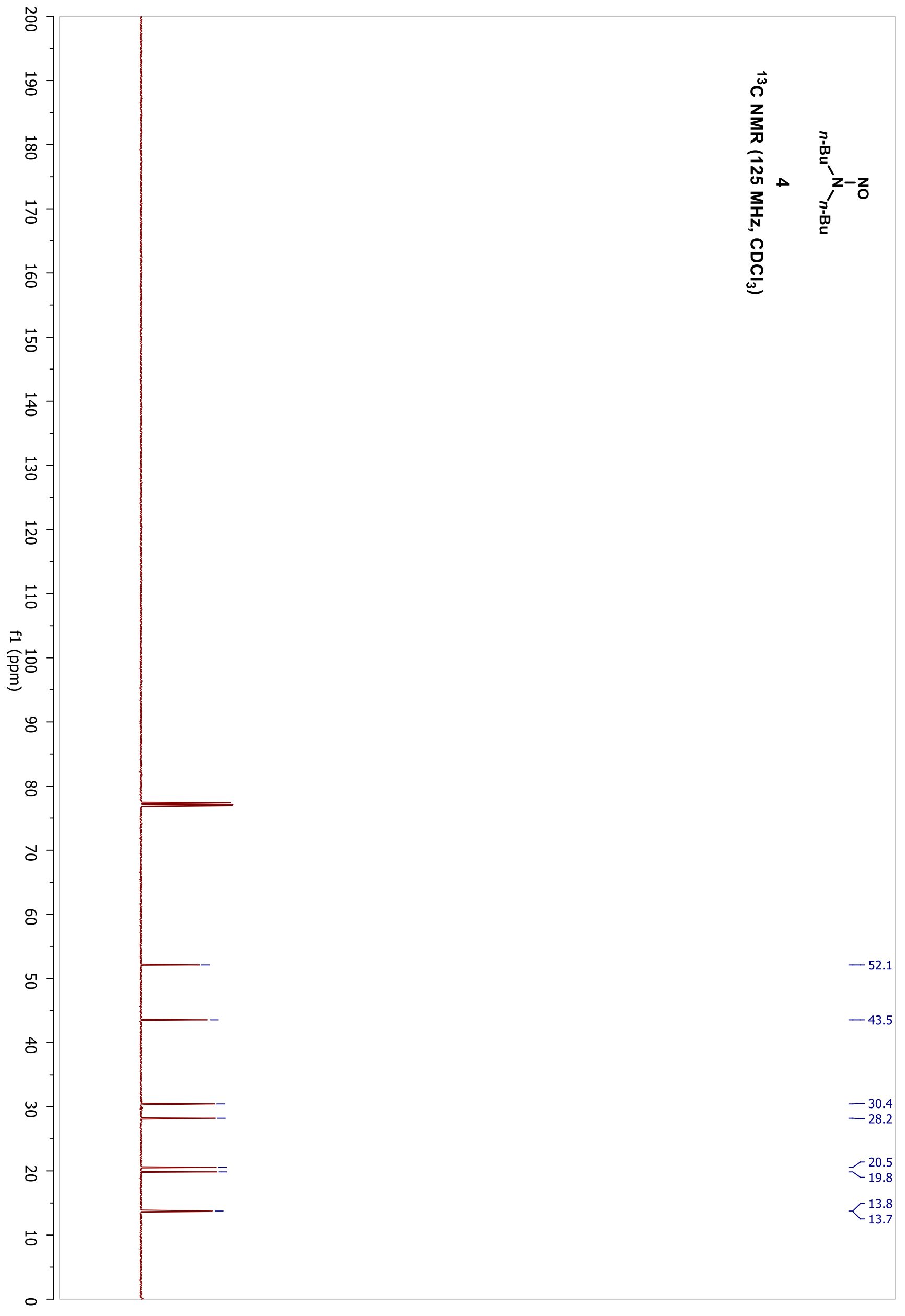




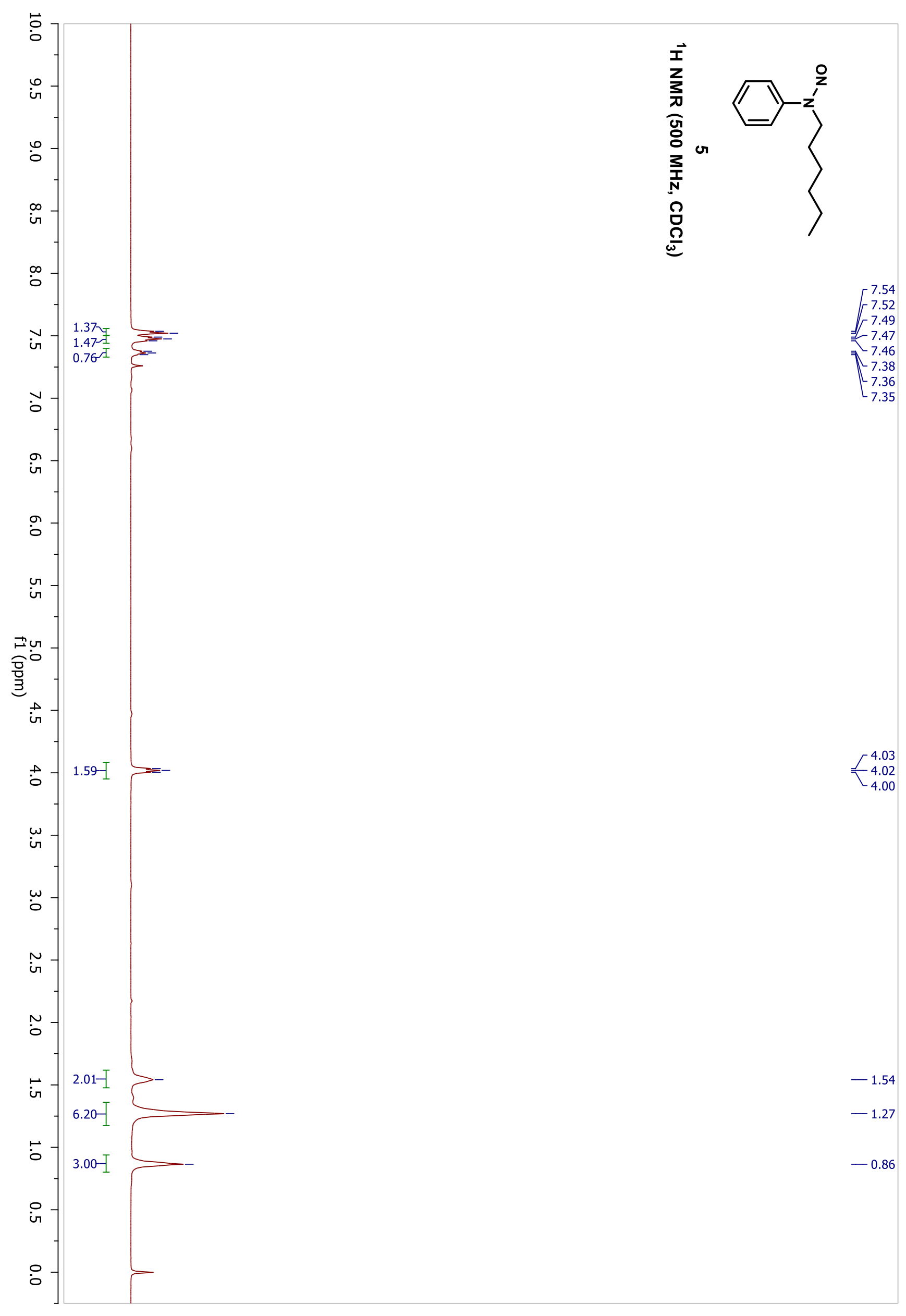




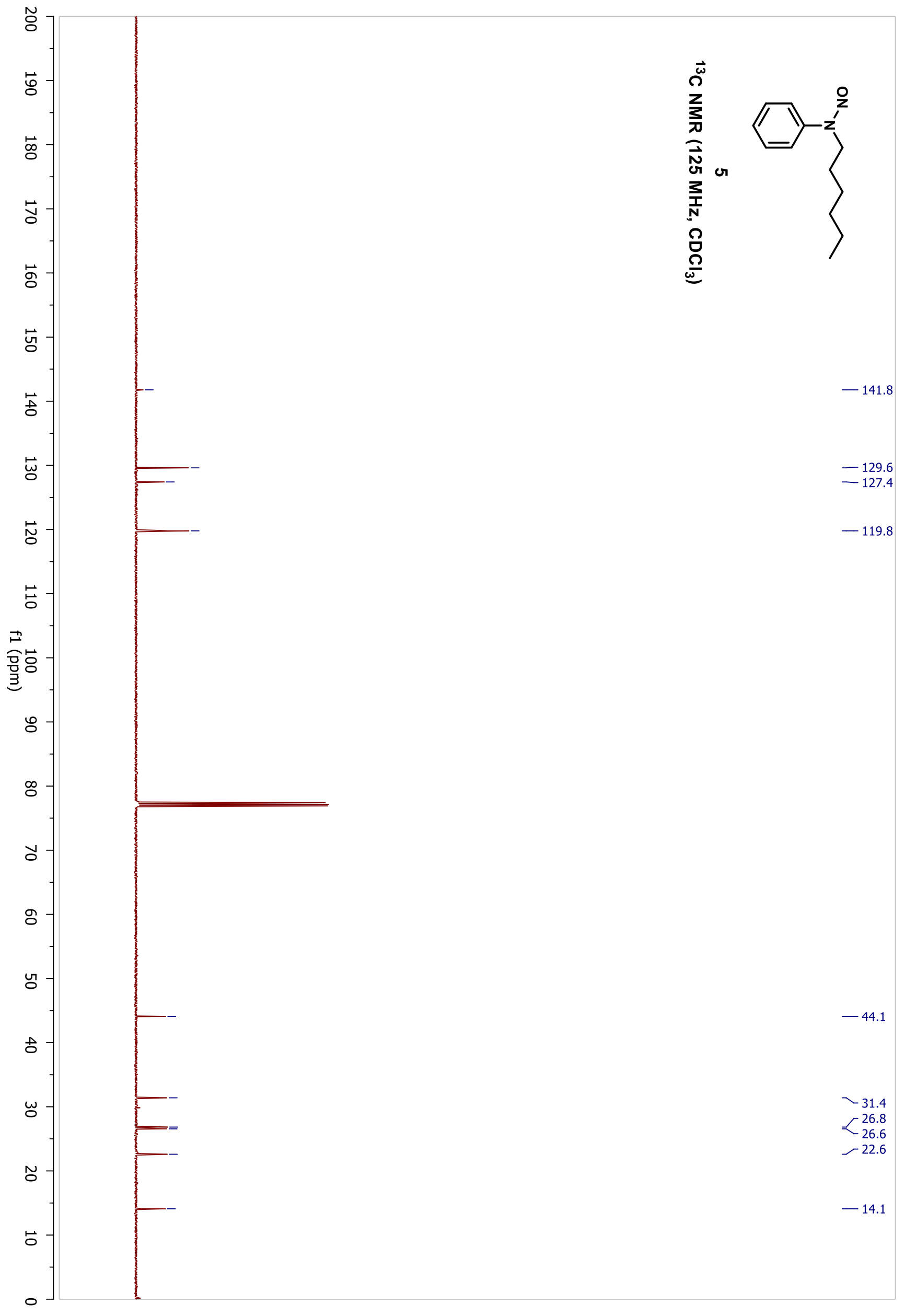




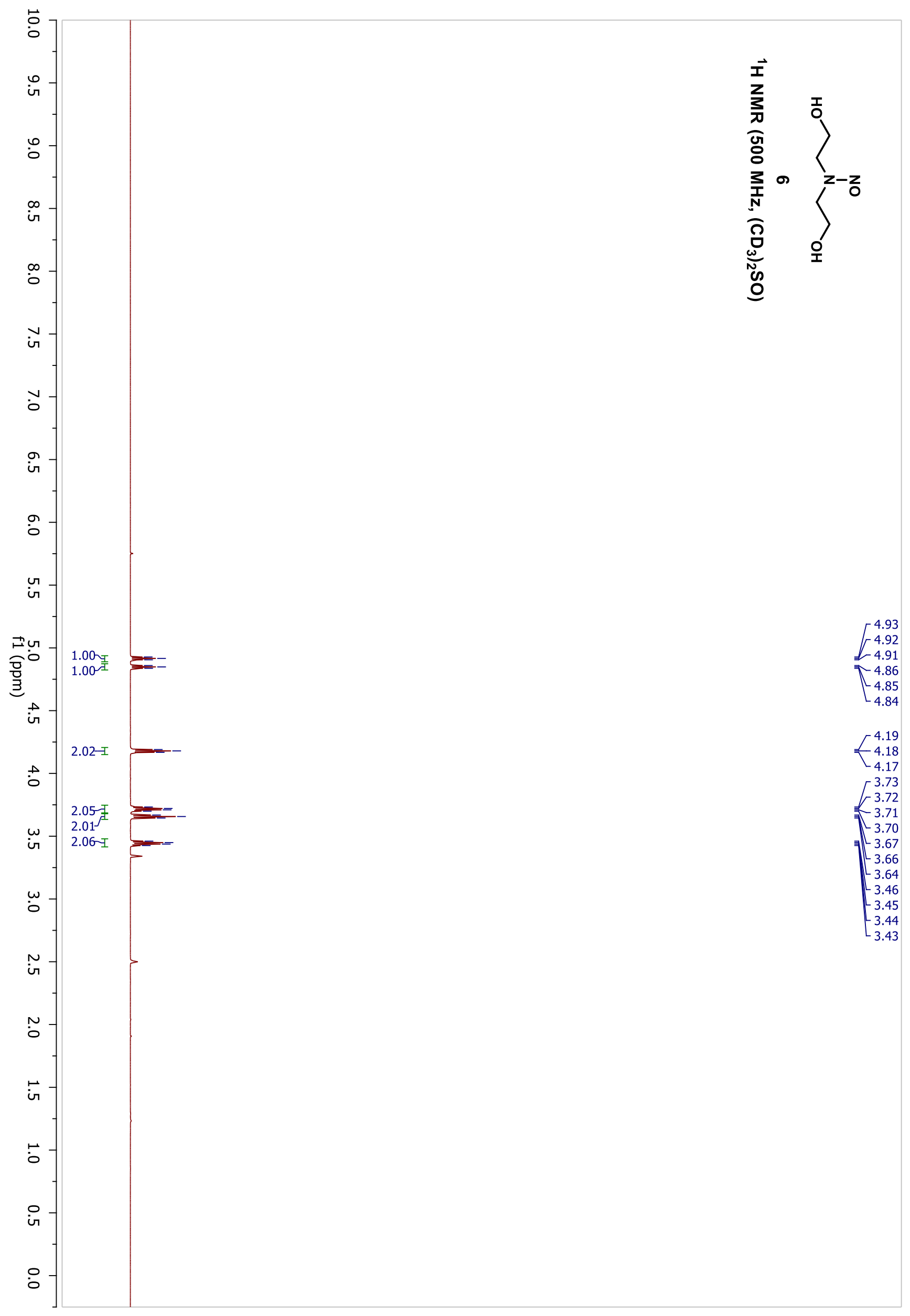




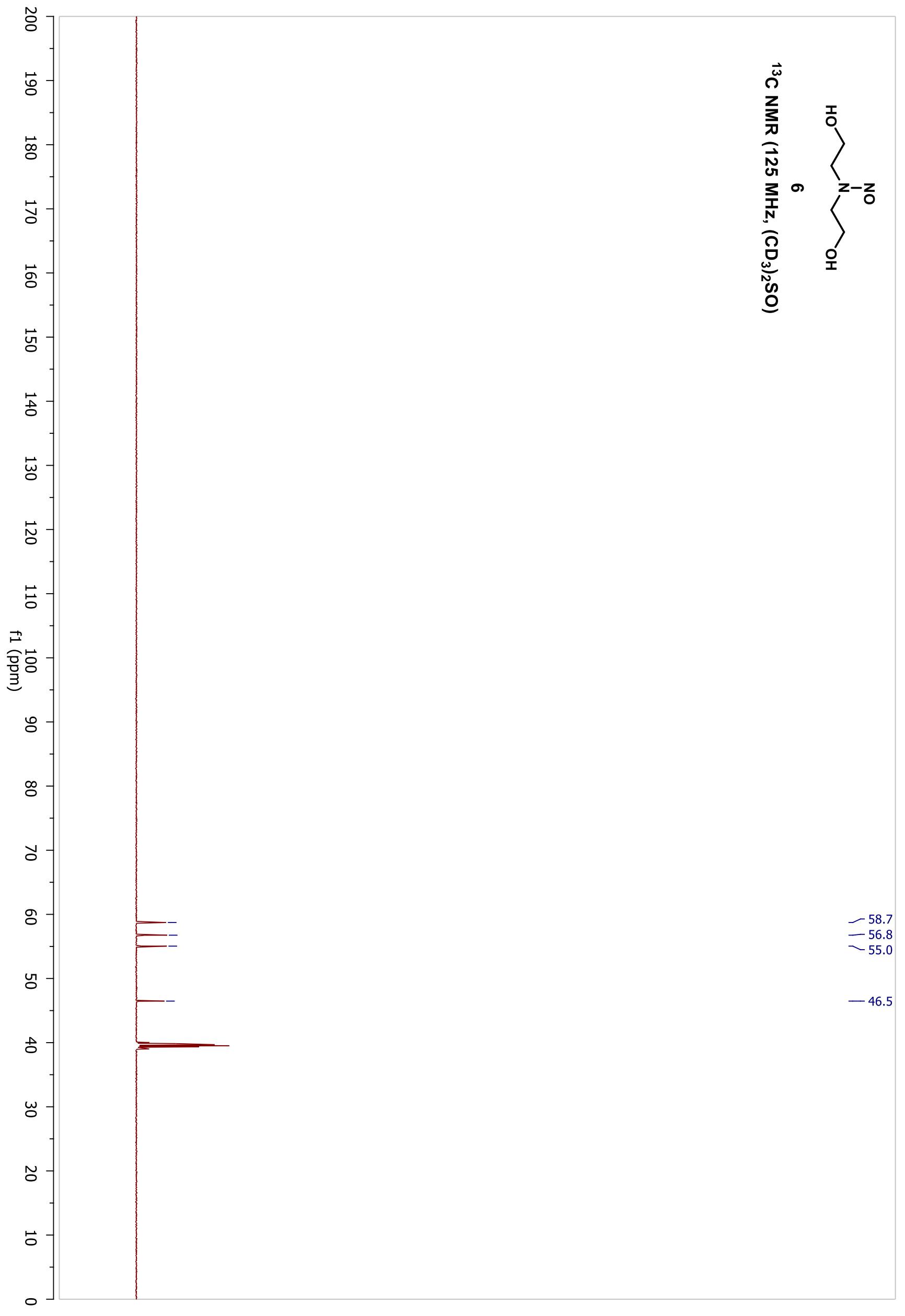




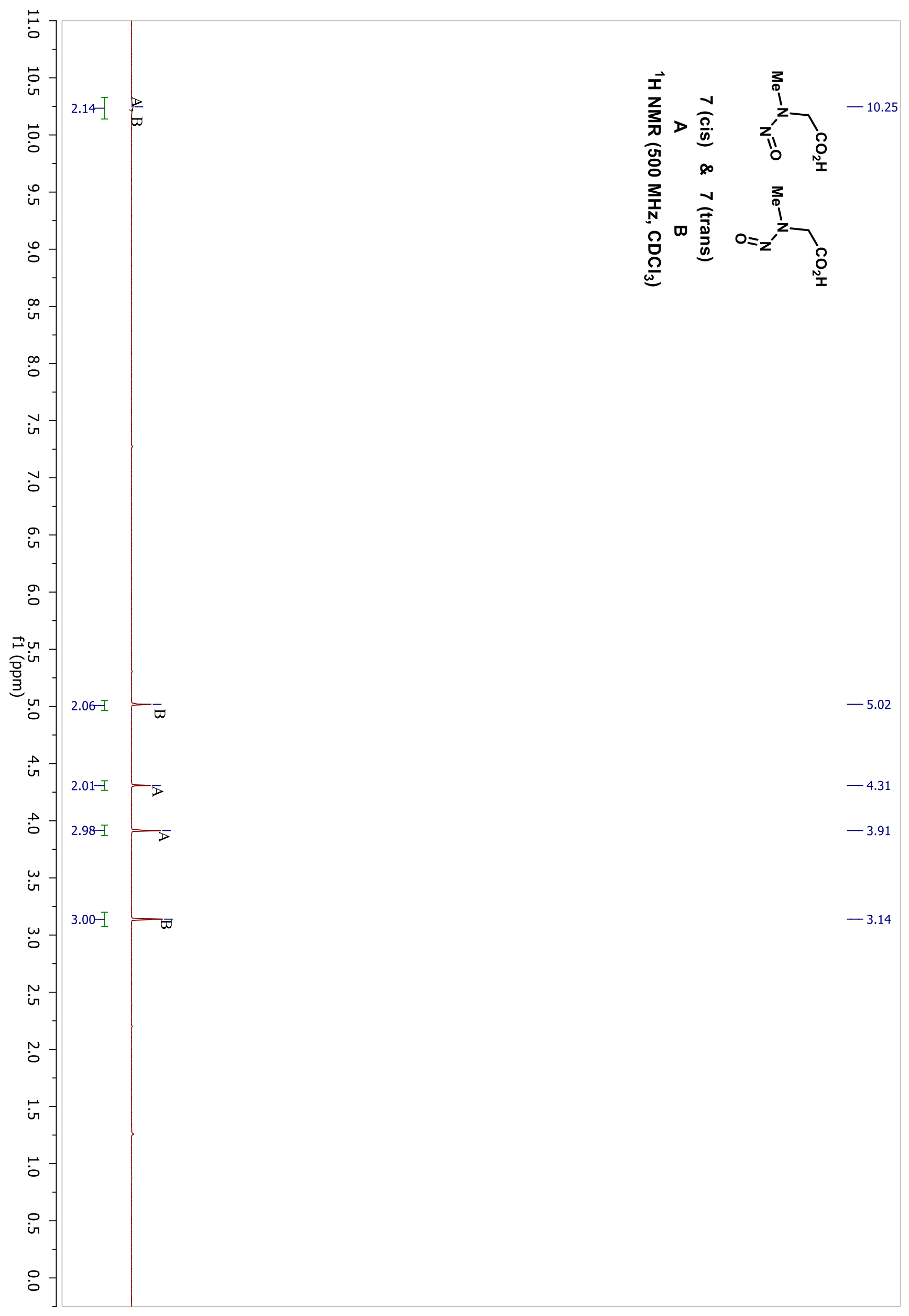




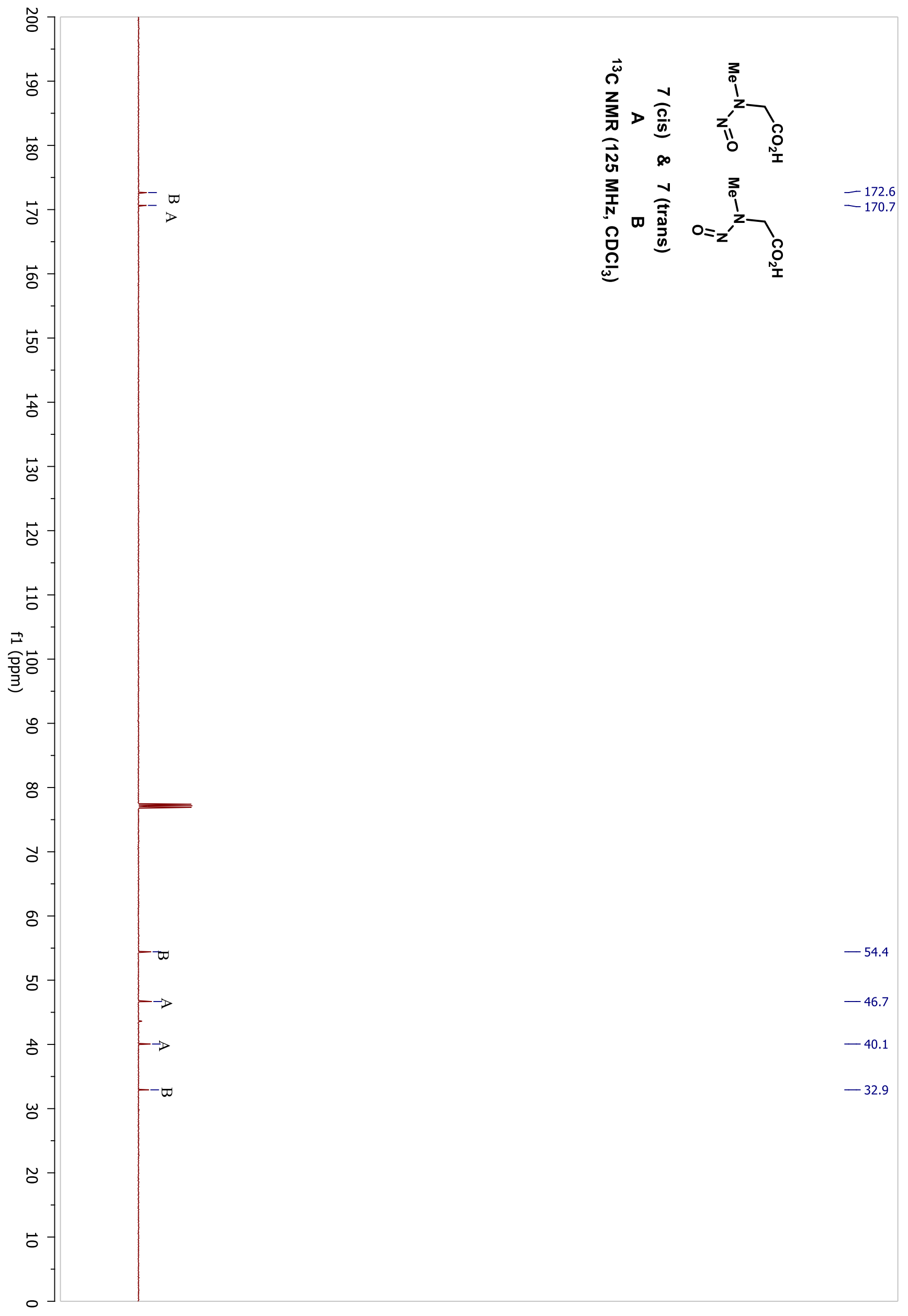




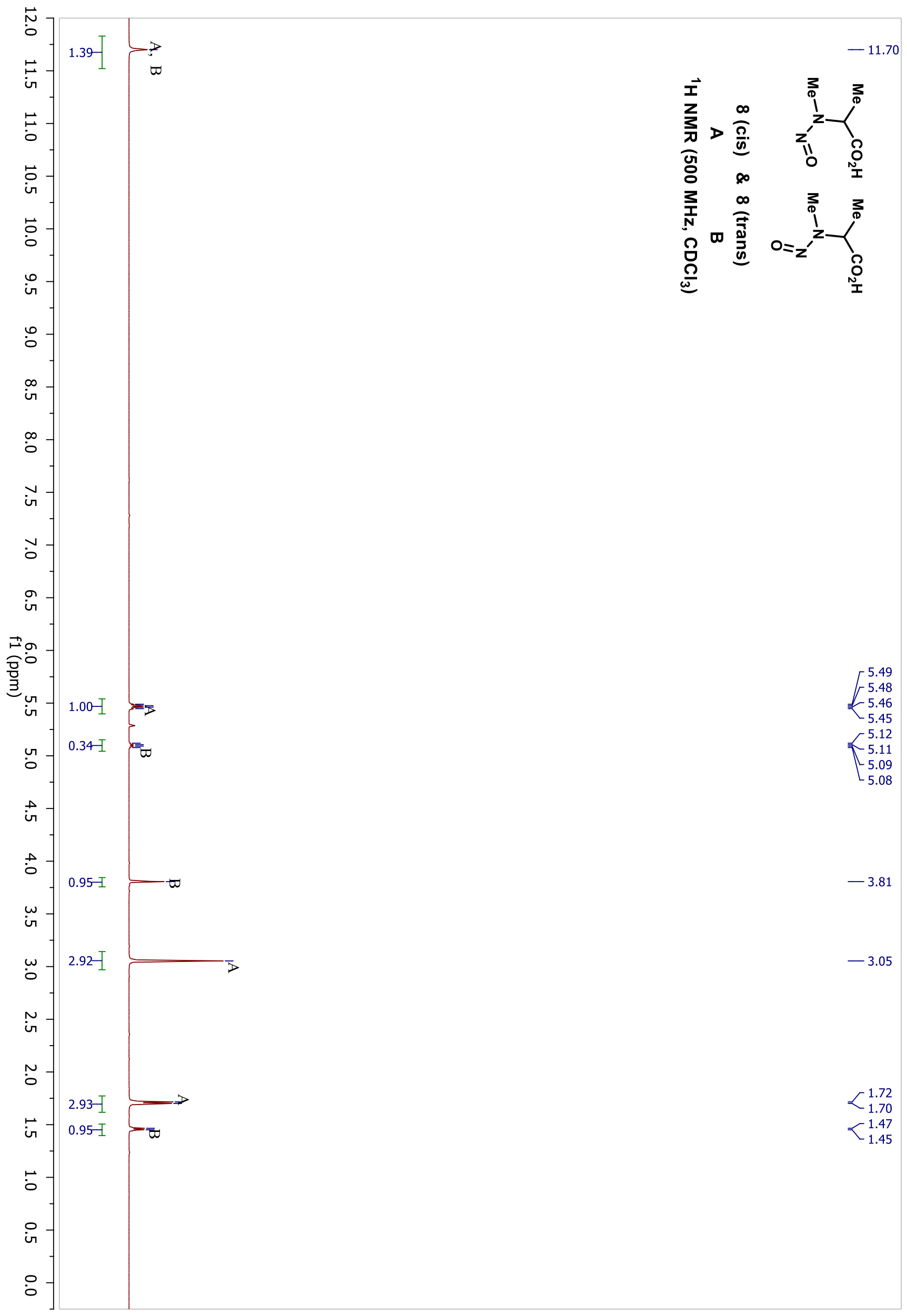




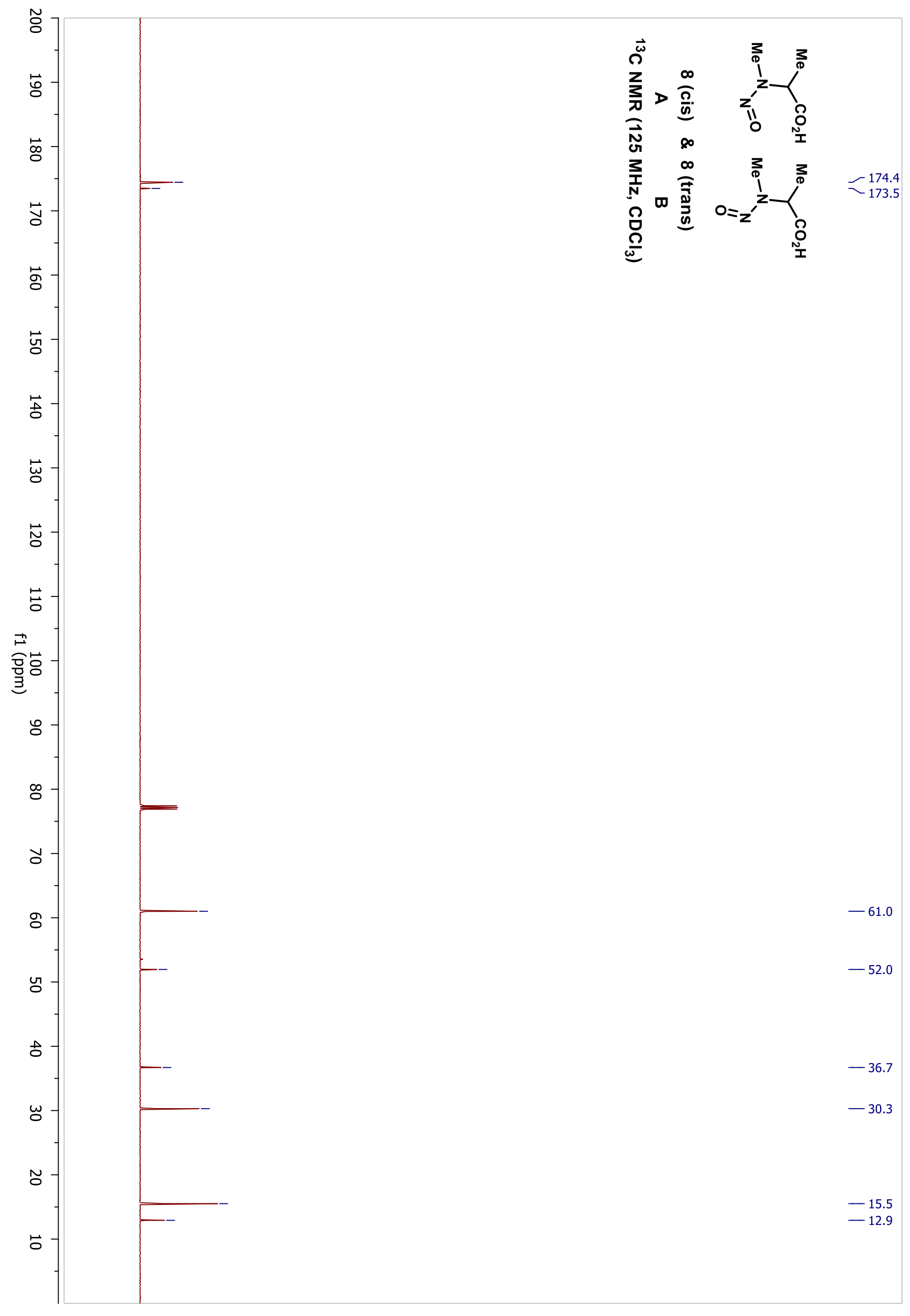




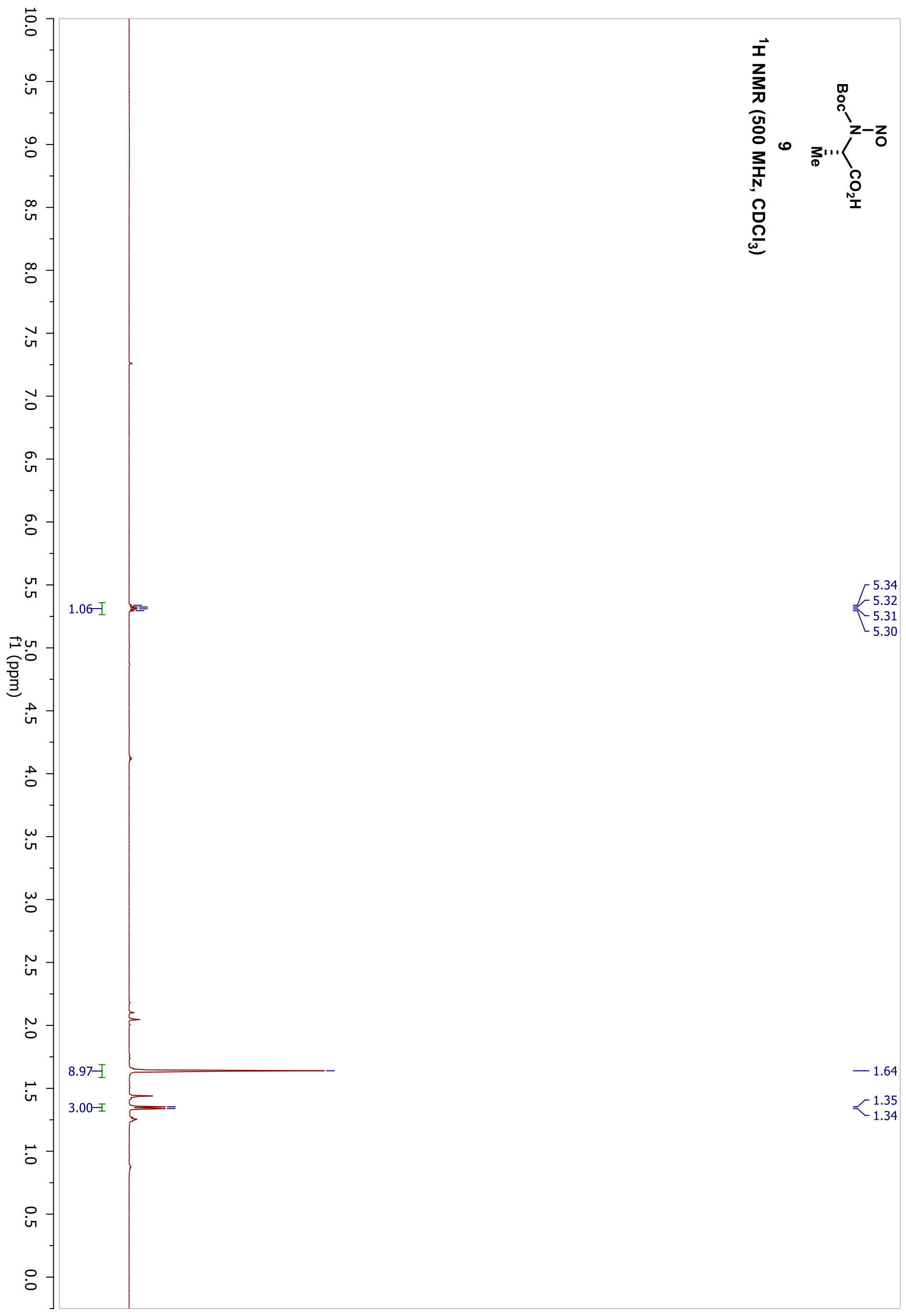




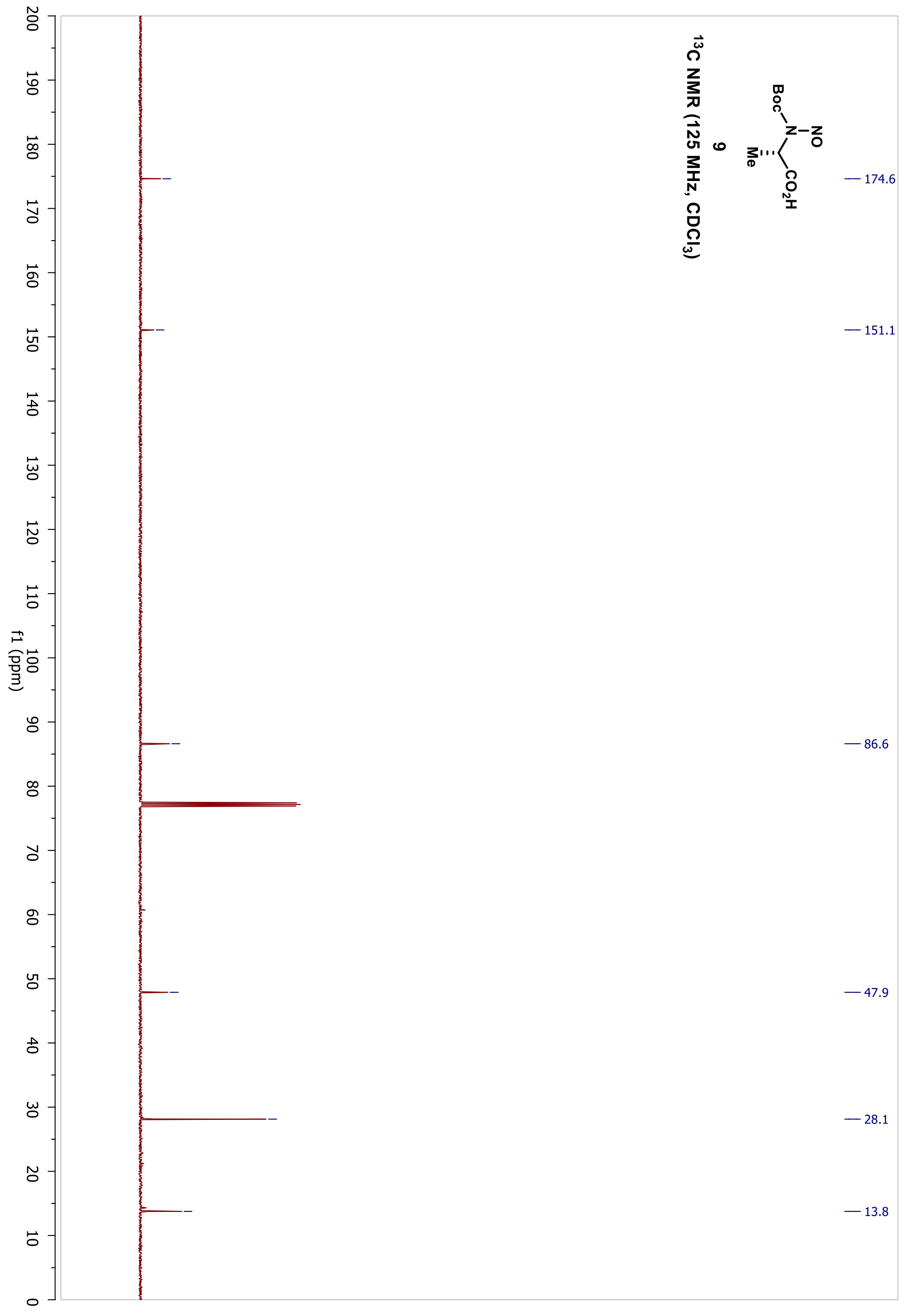




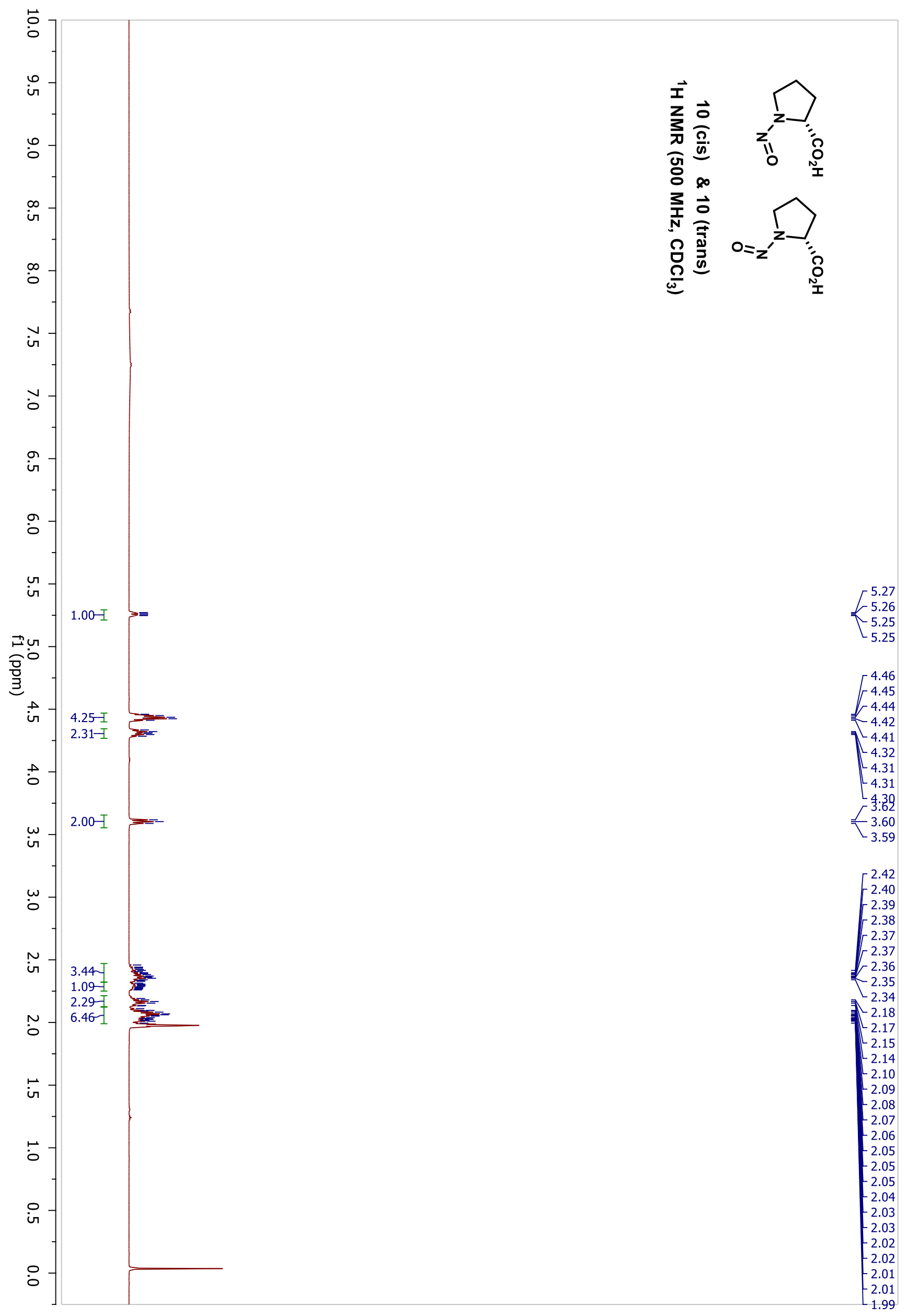

ڤ̊ 


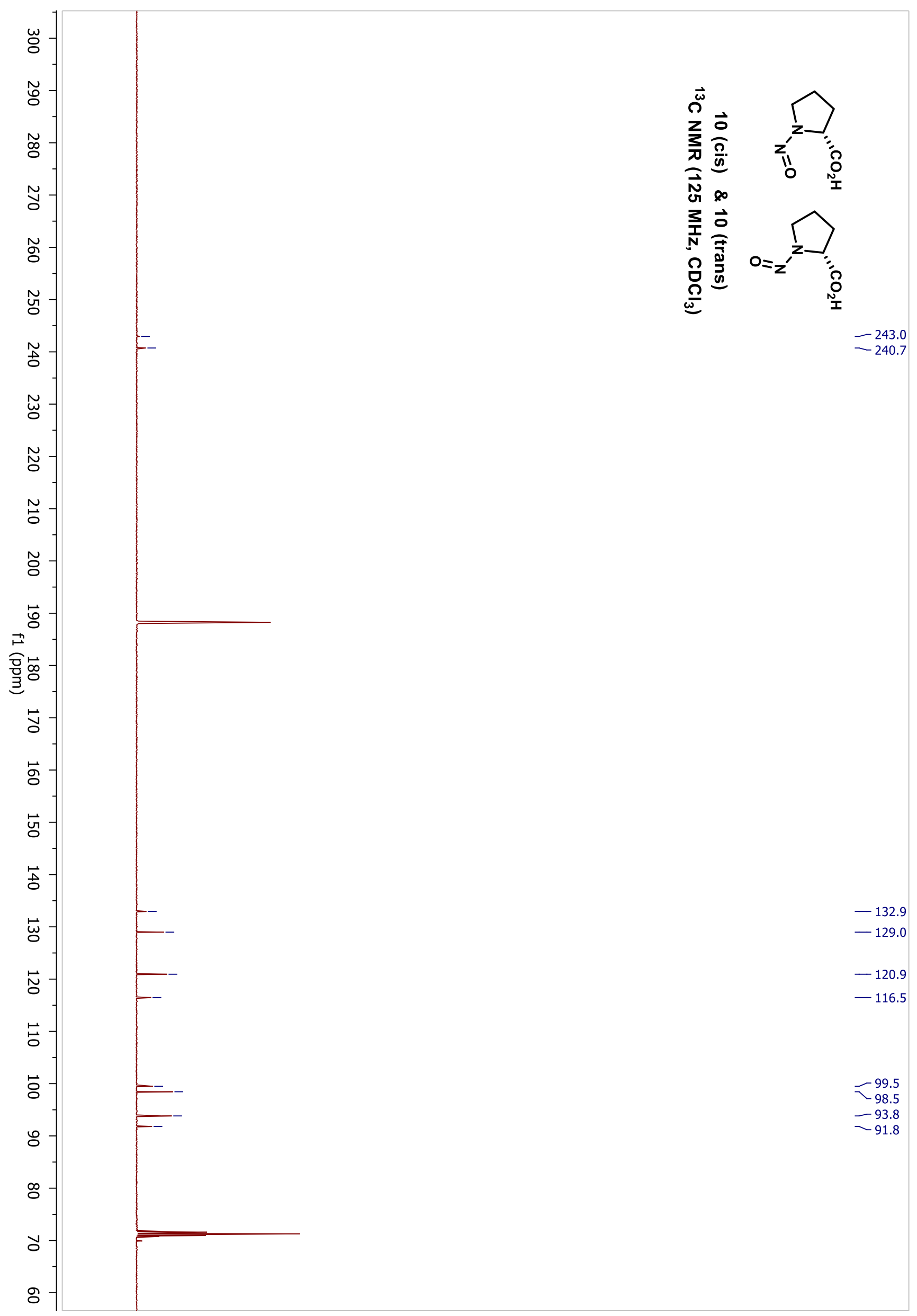




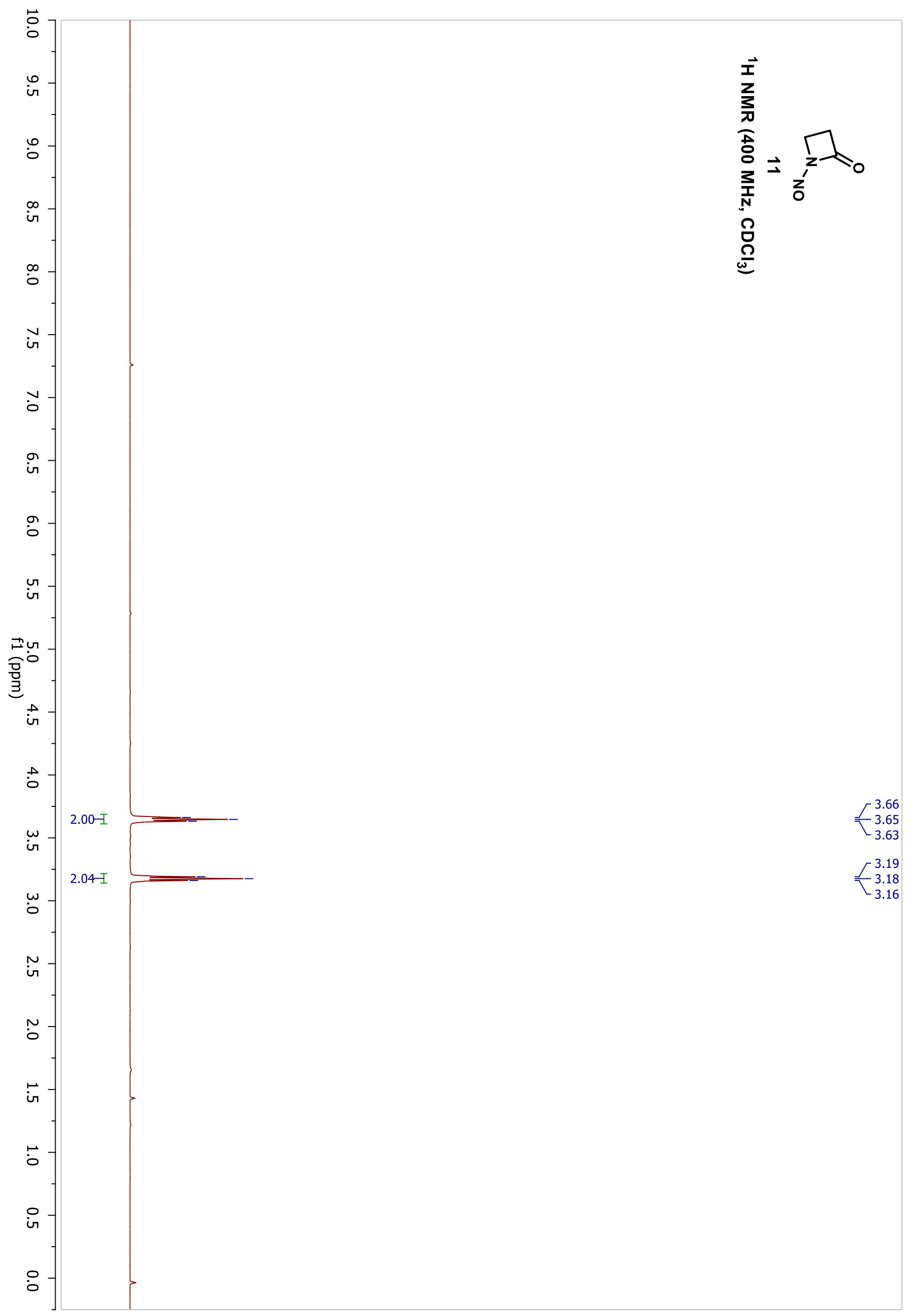




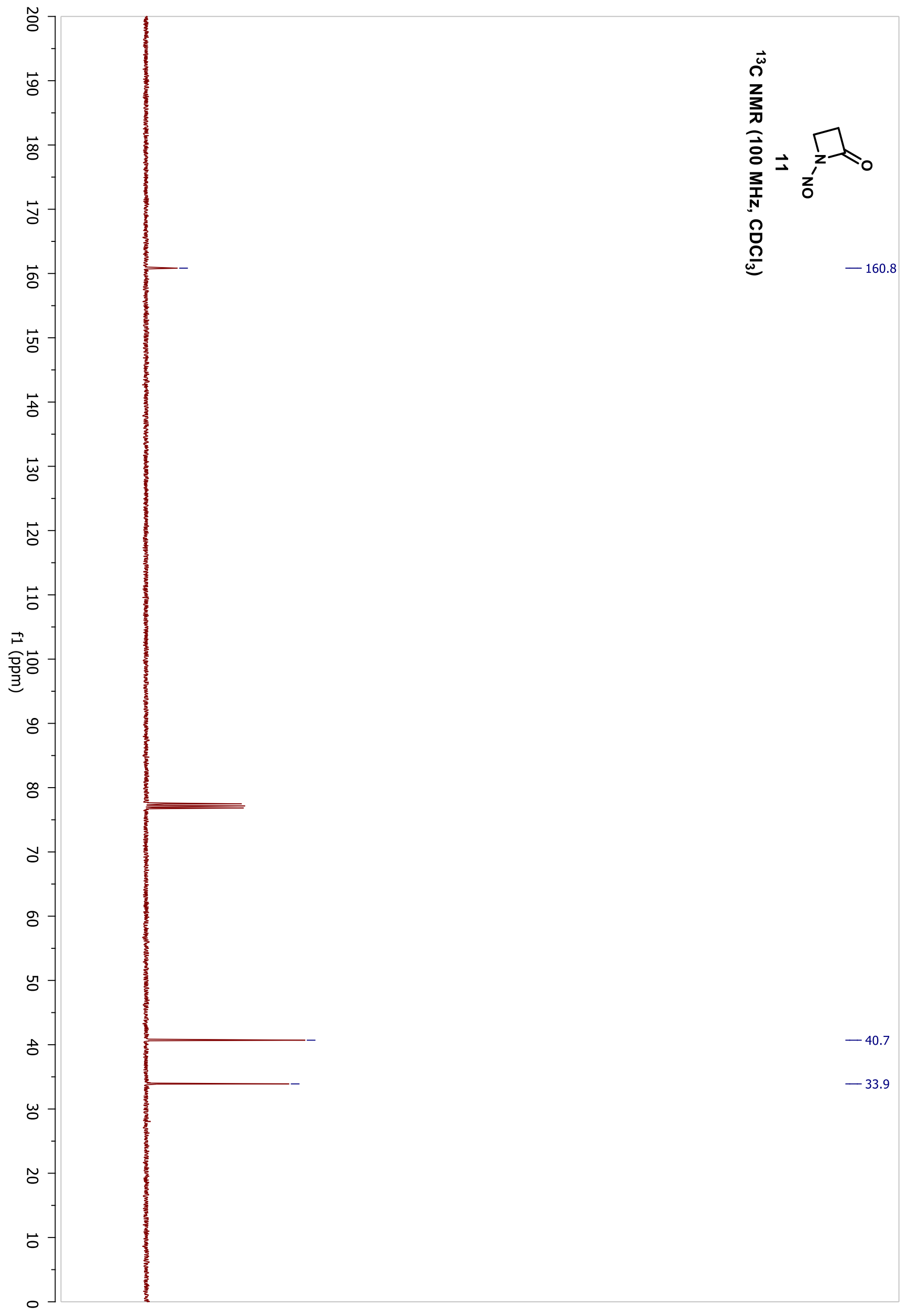




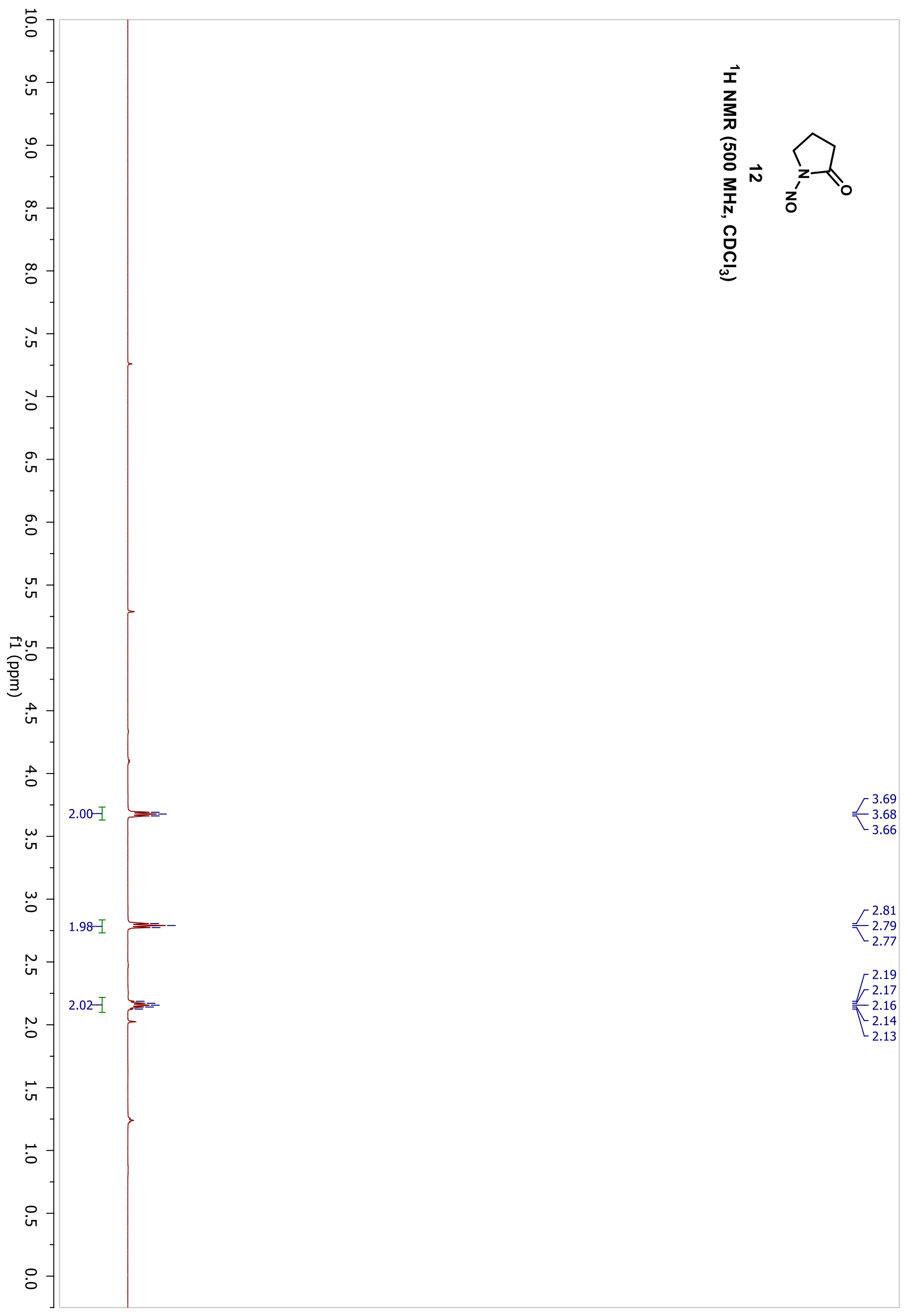




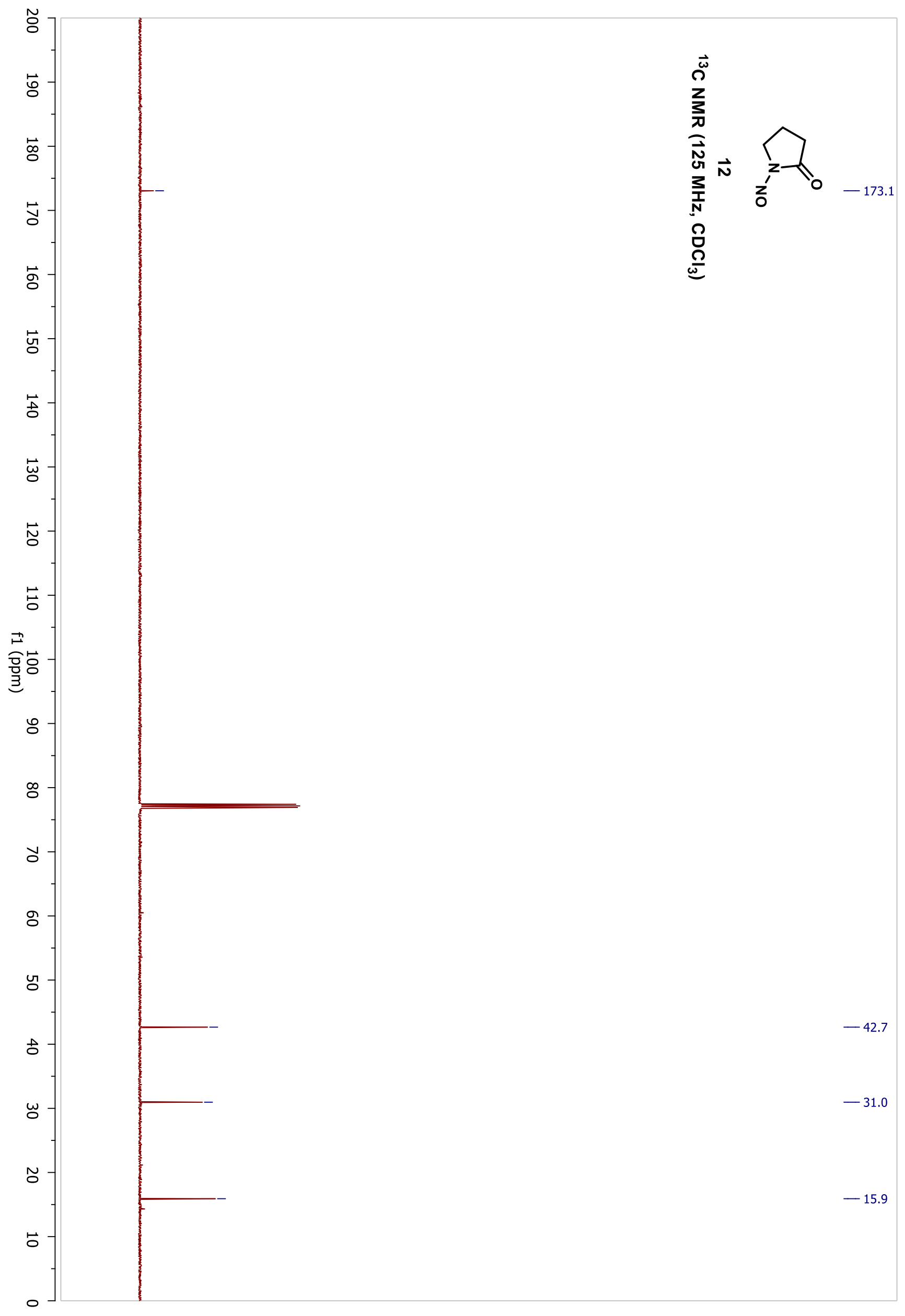




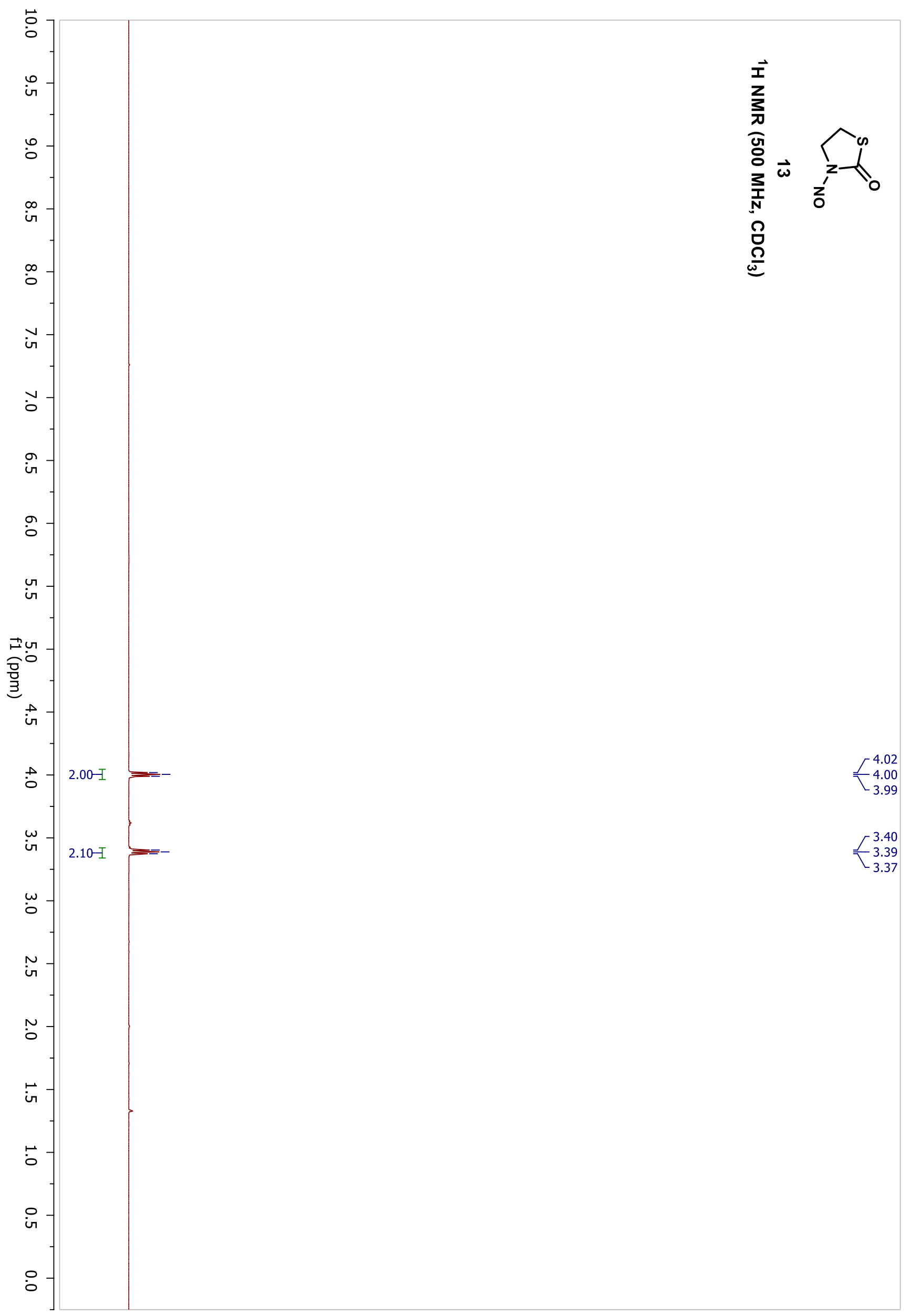




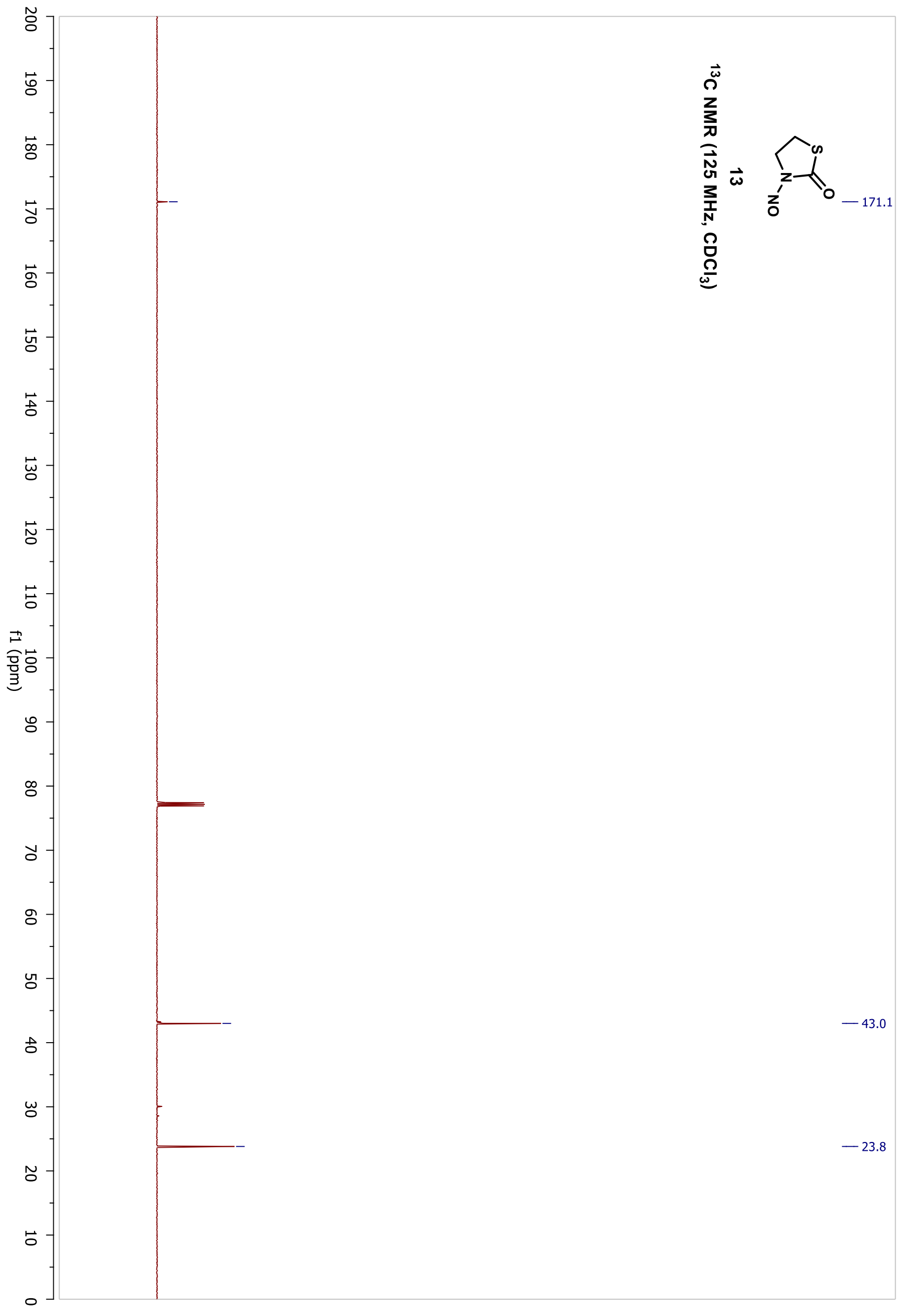




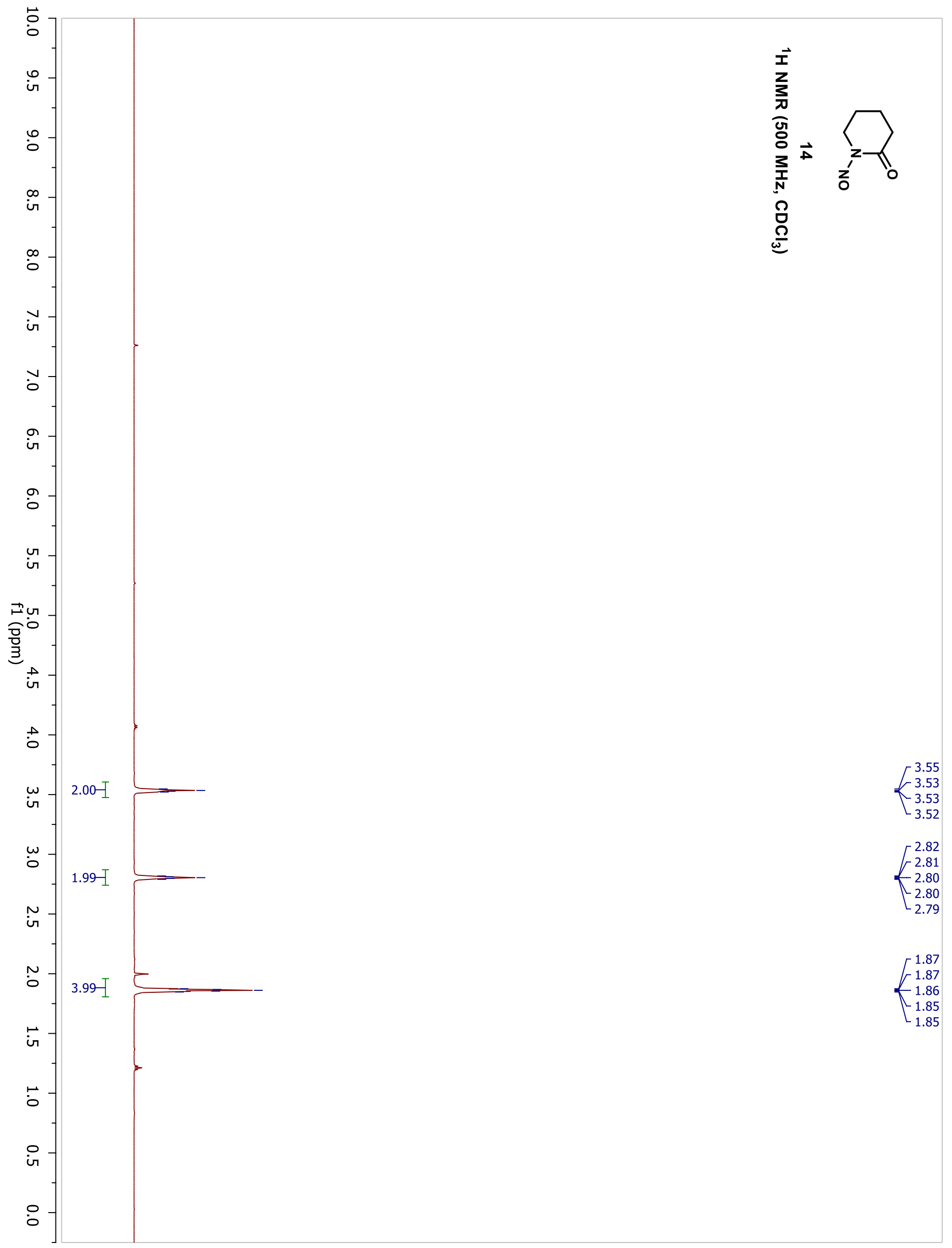




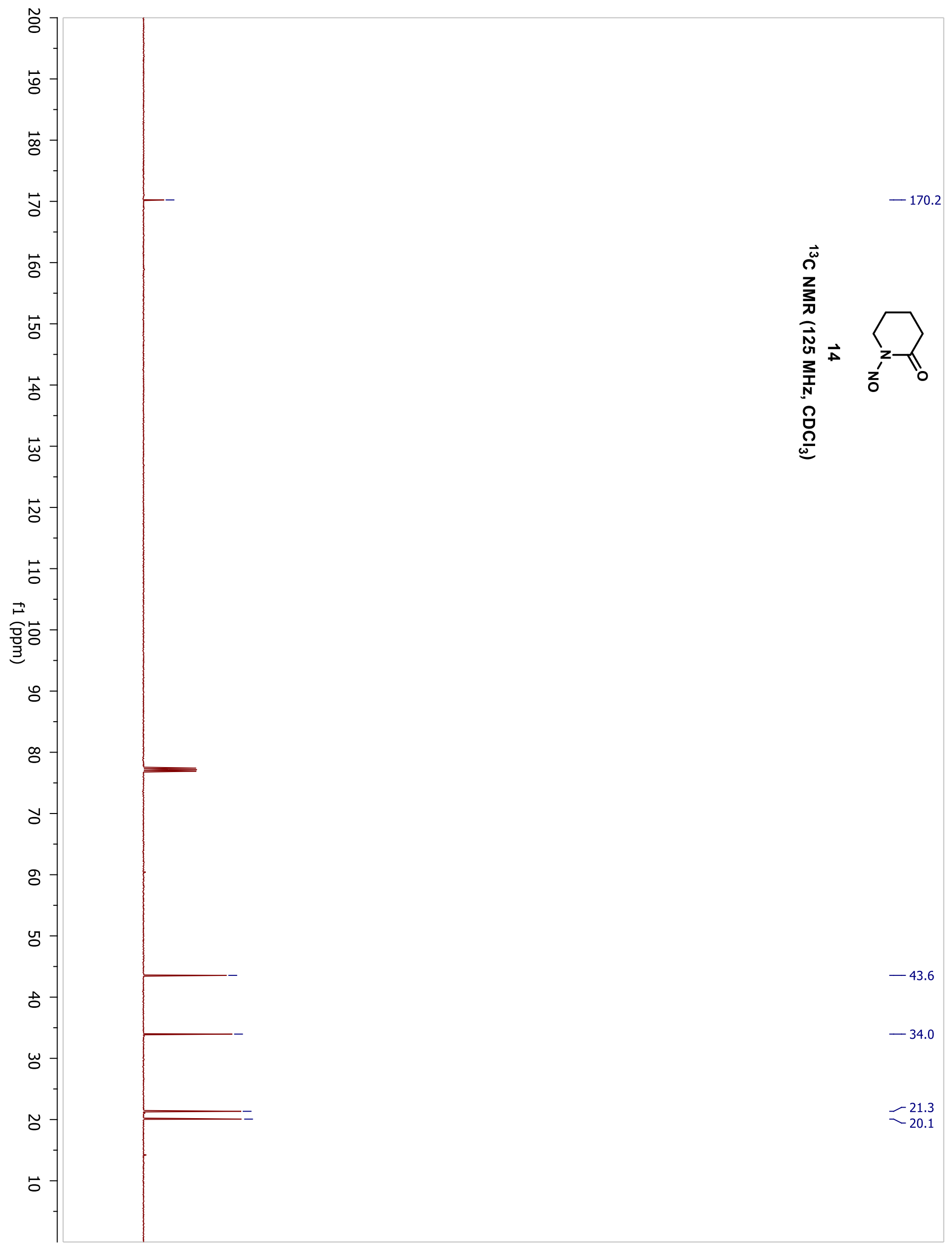




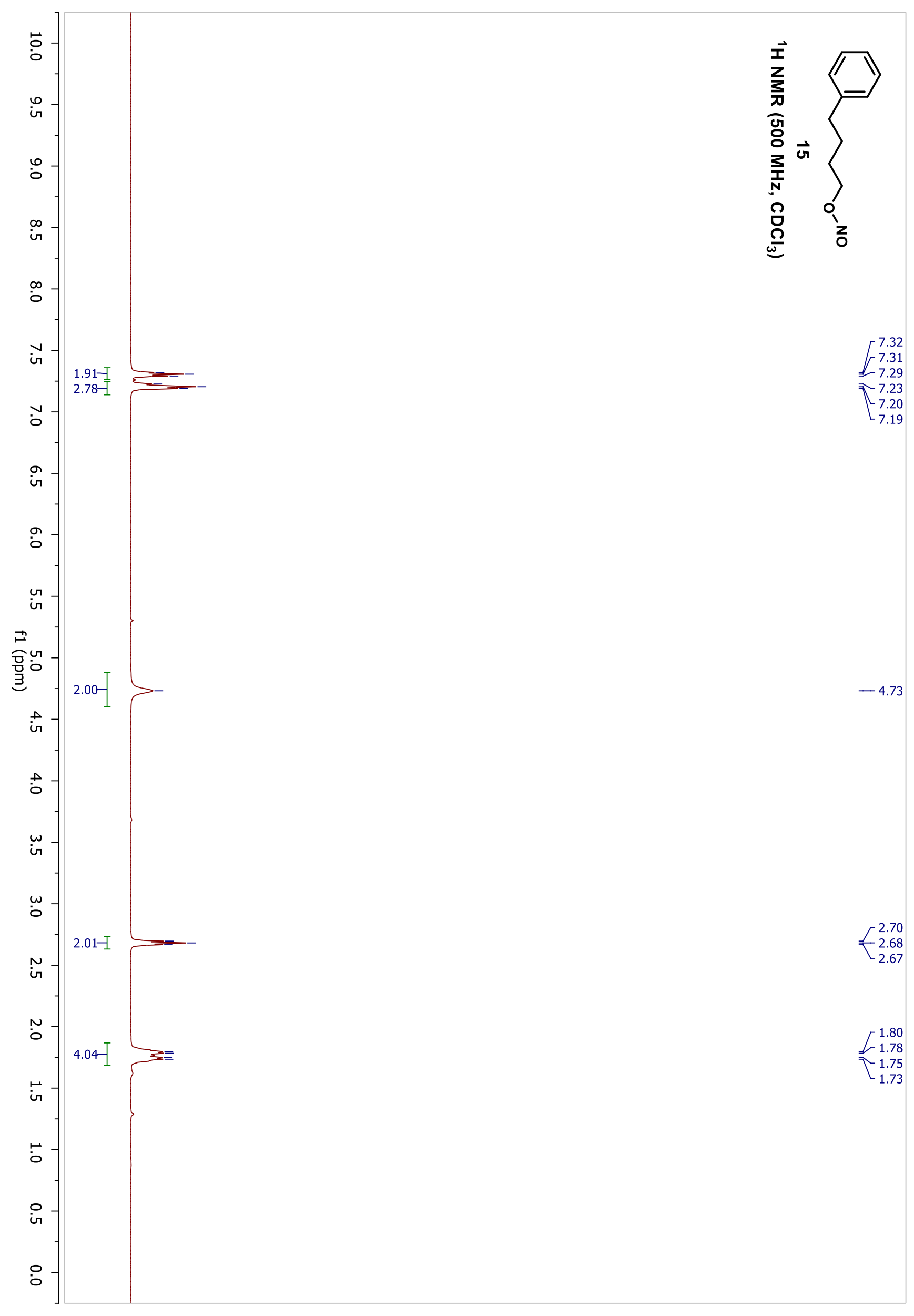




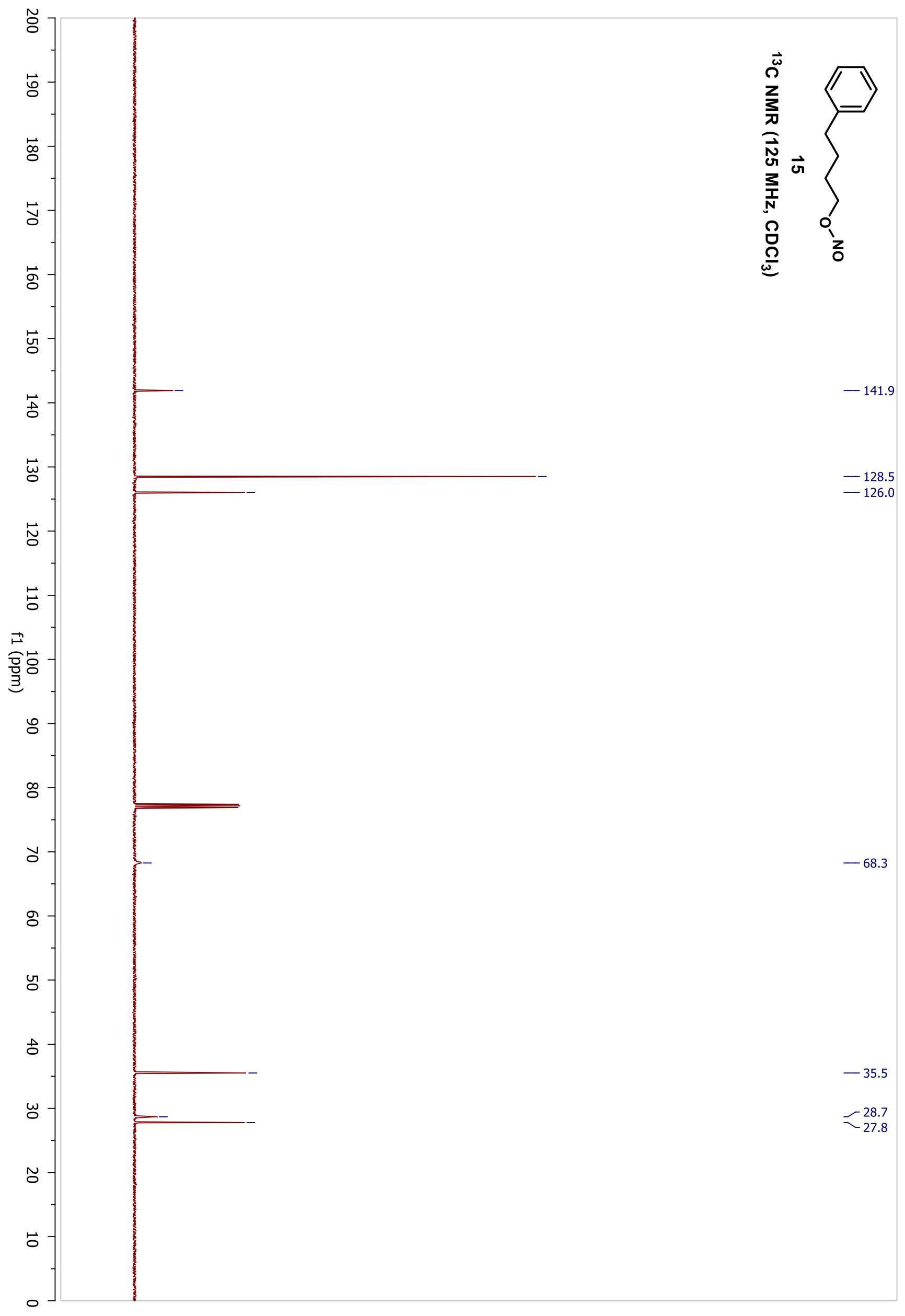



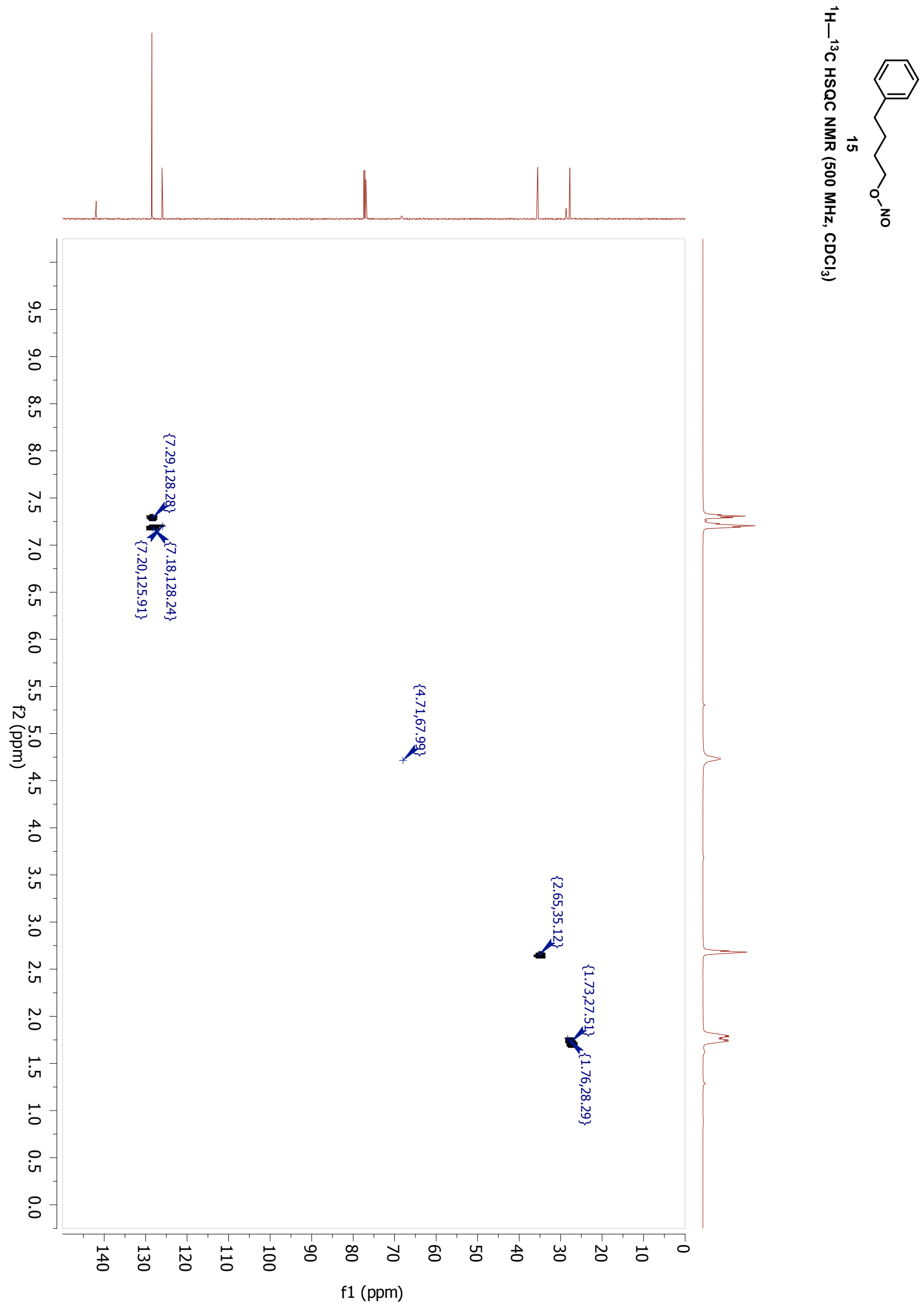


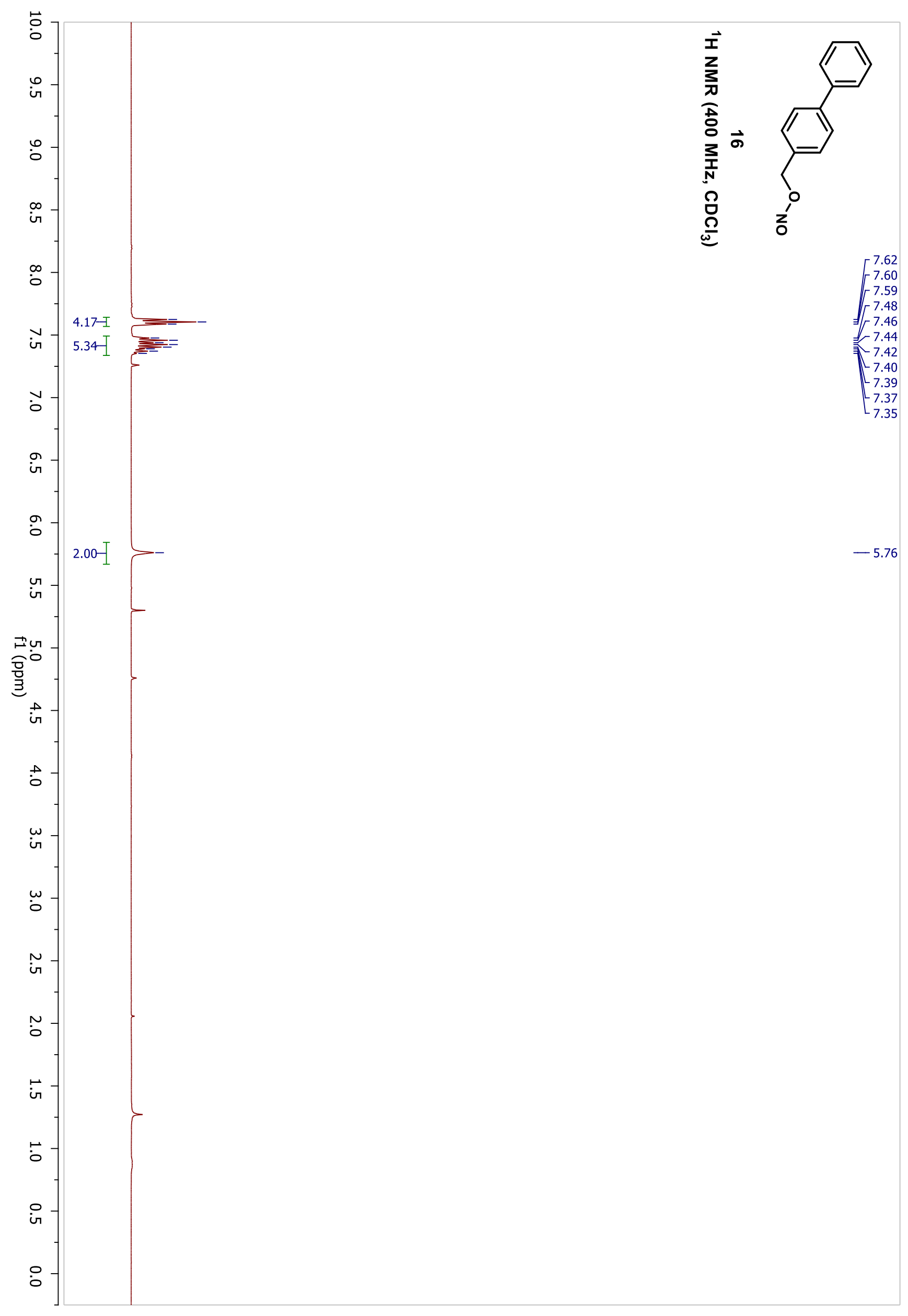




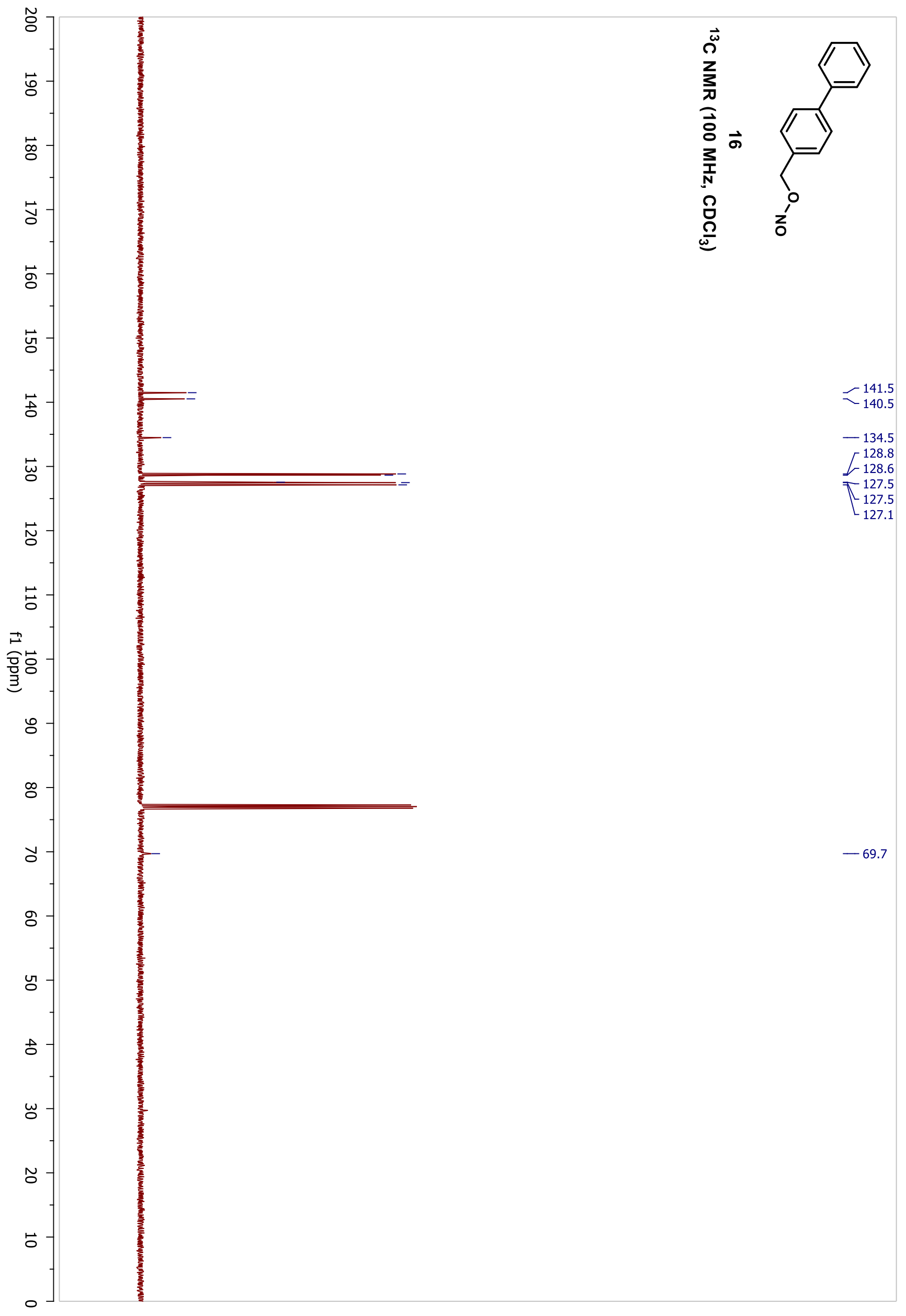




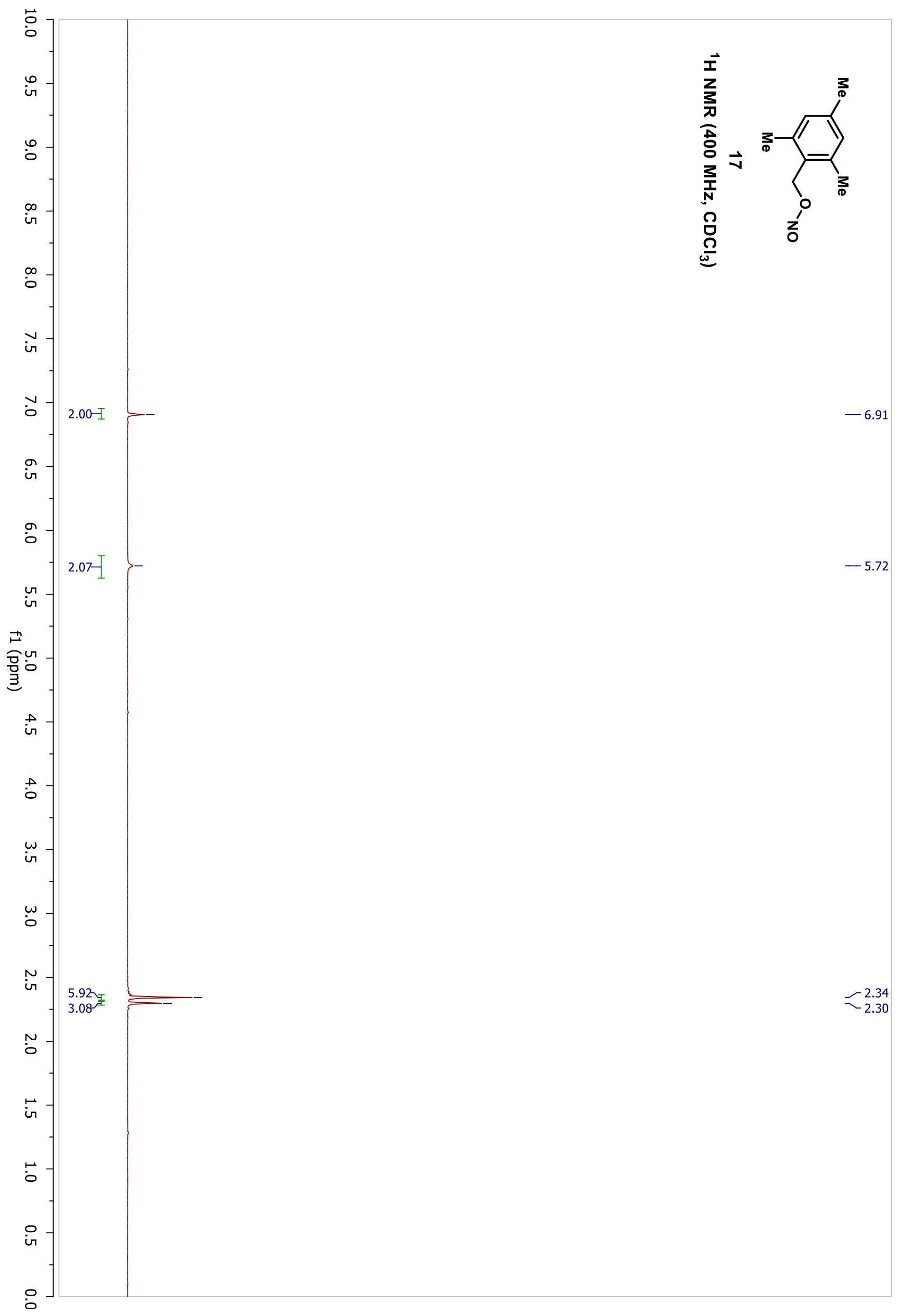




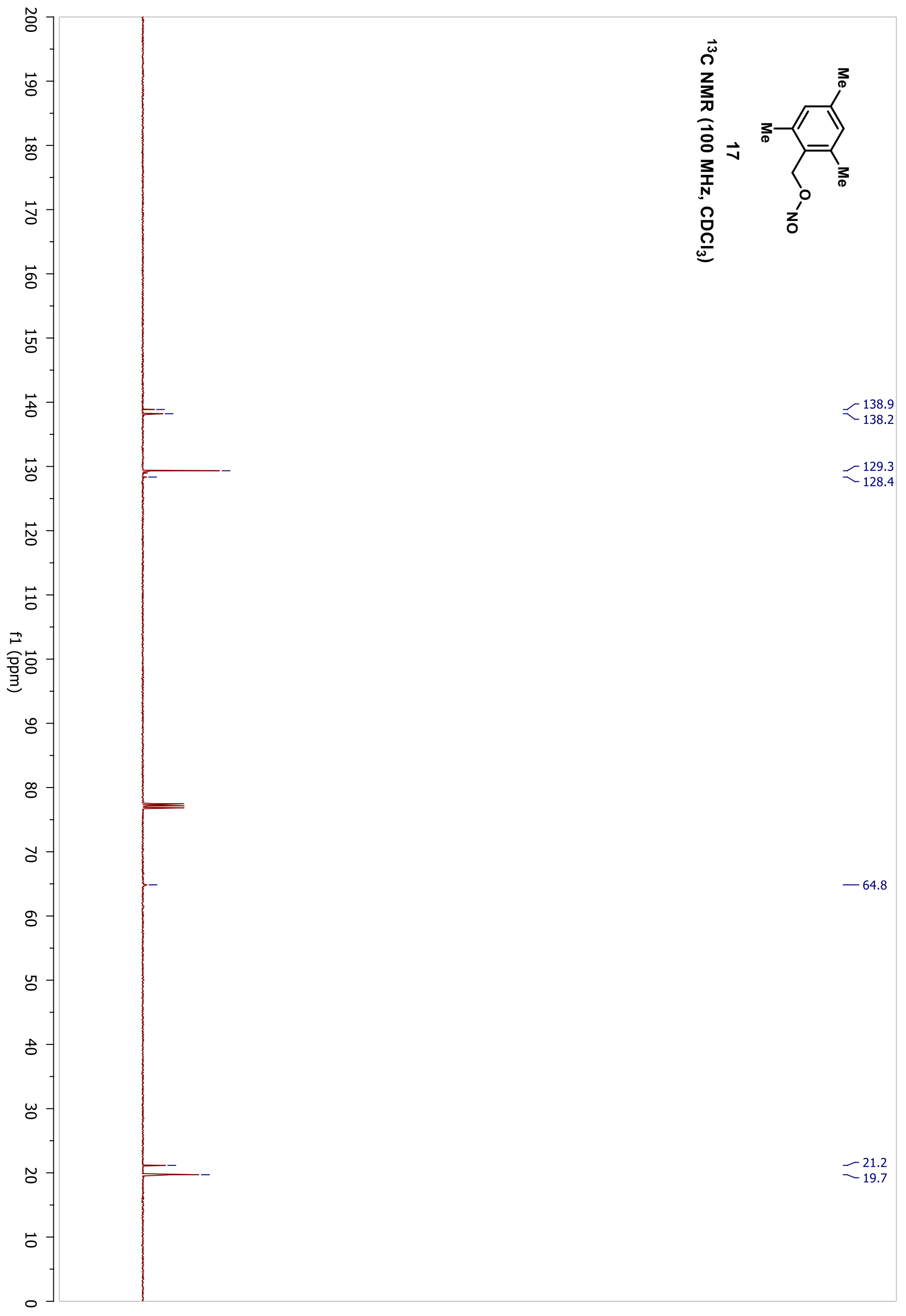




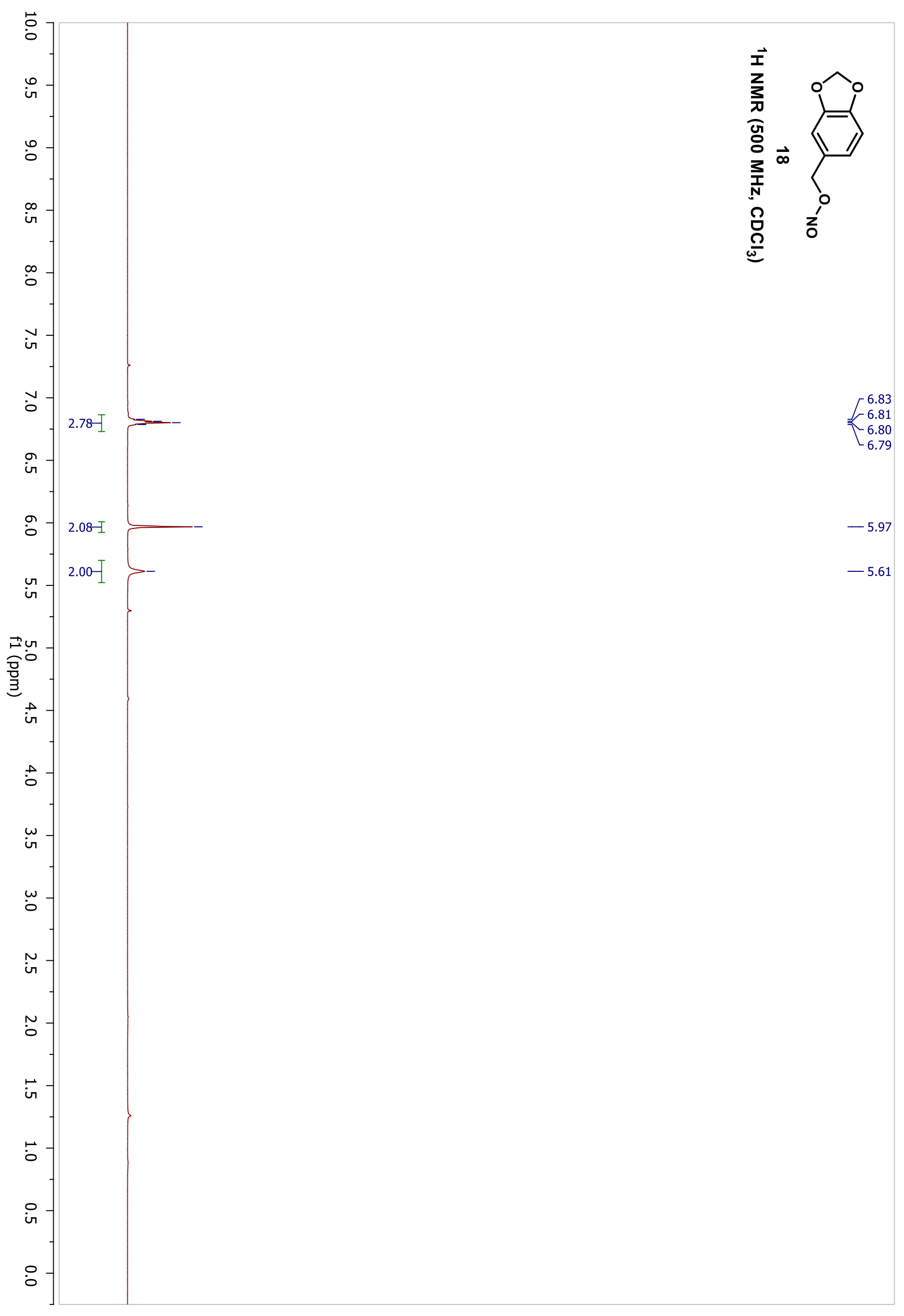




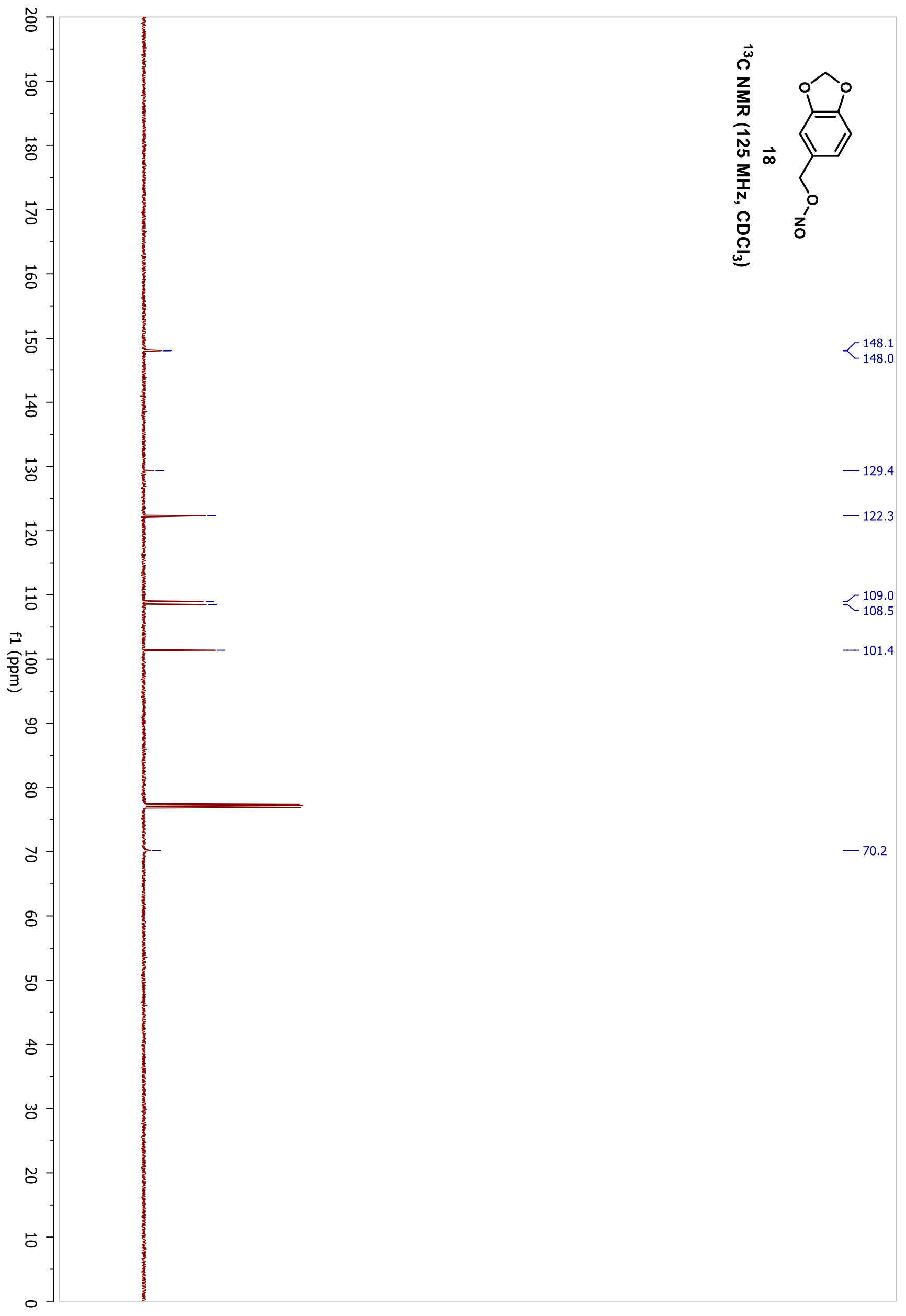




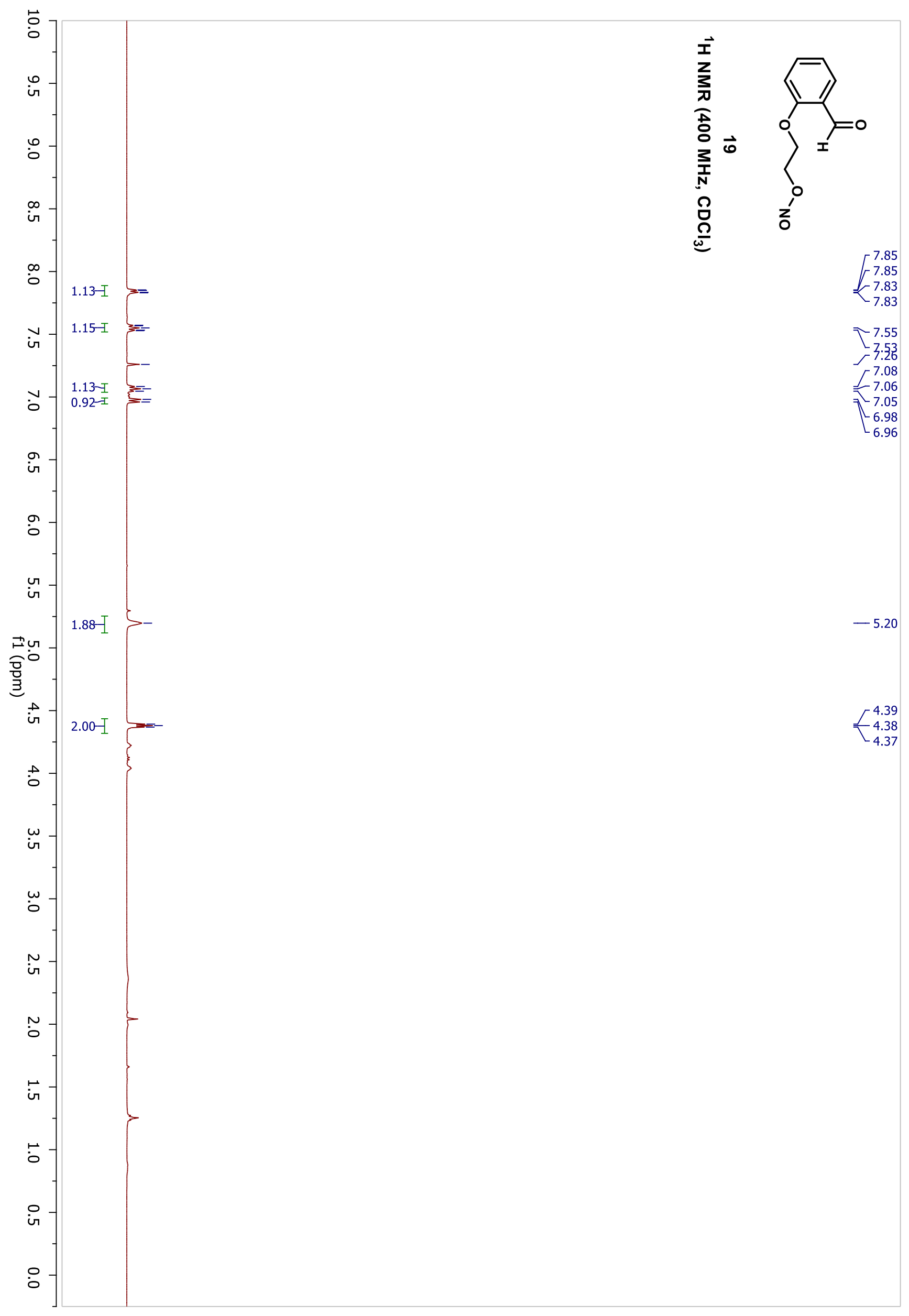




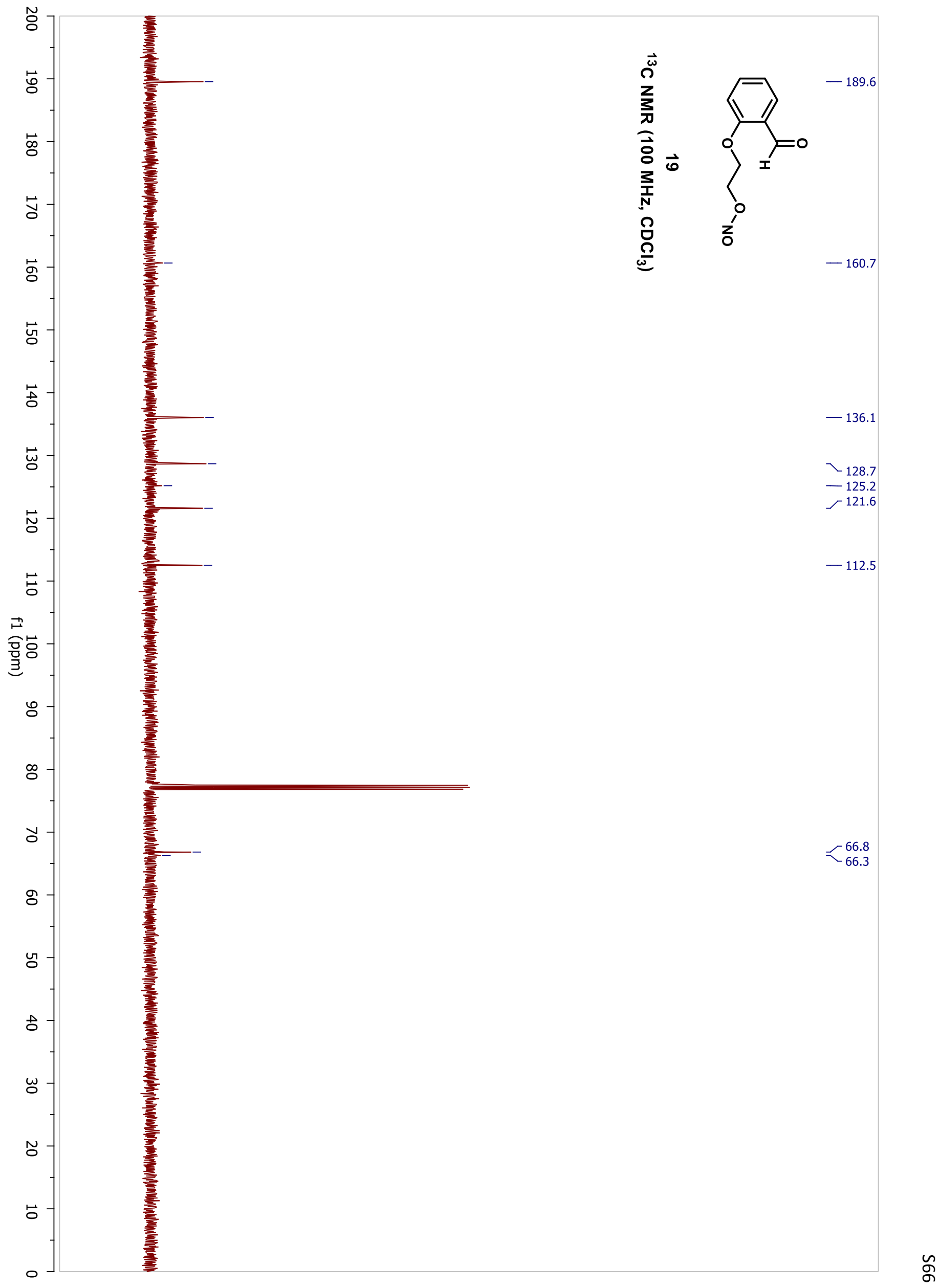




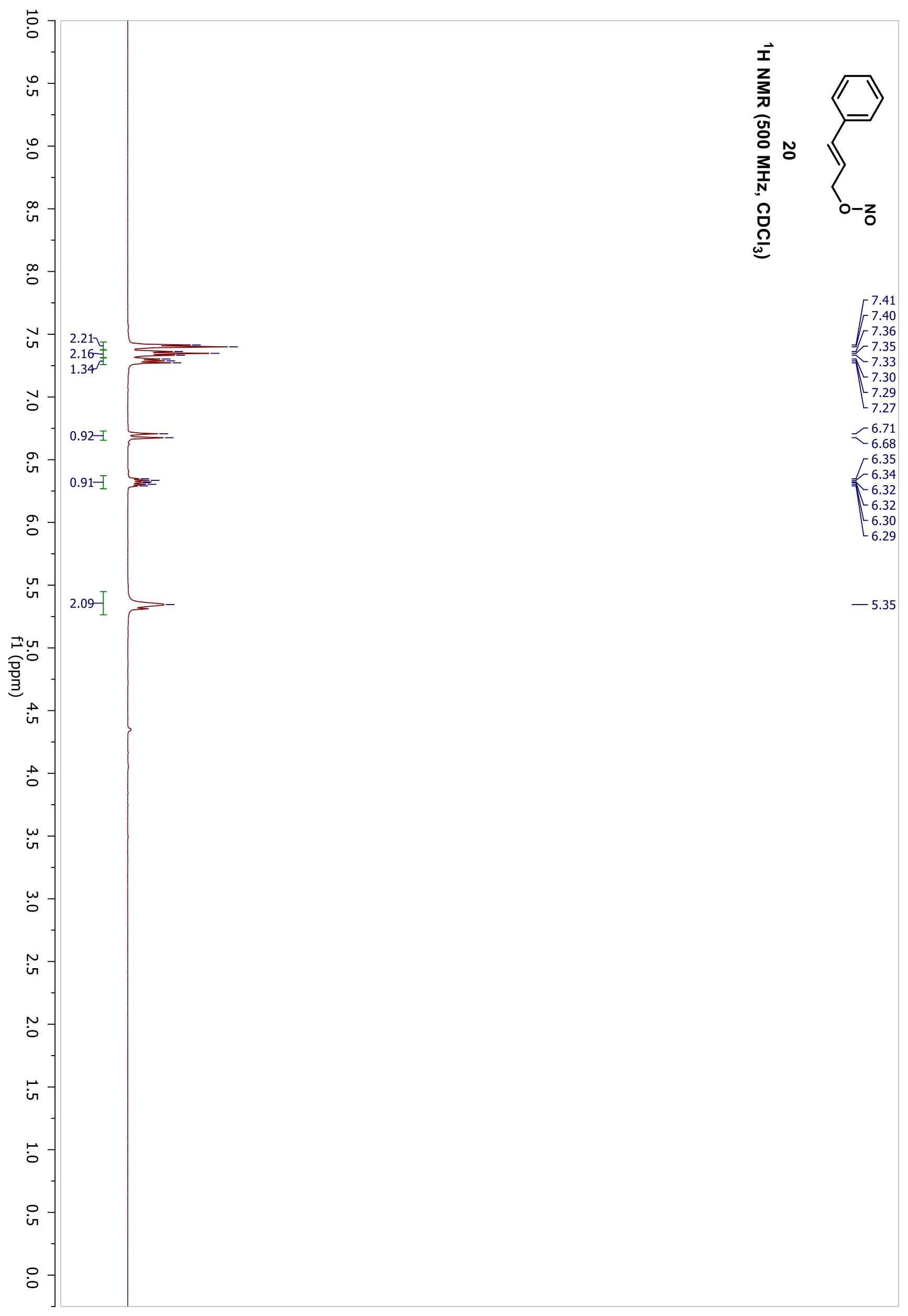




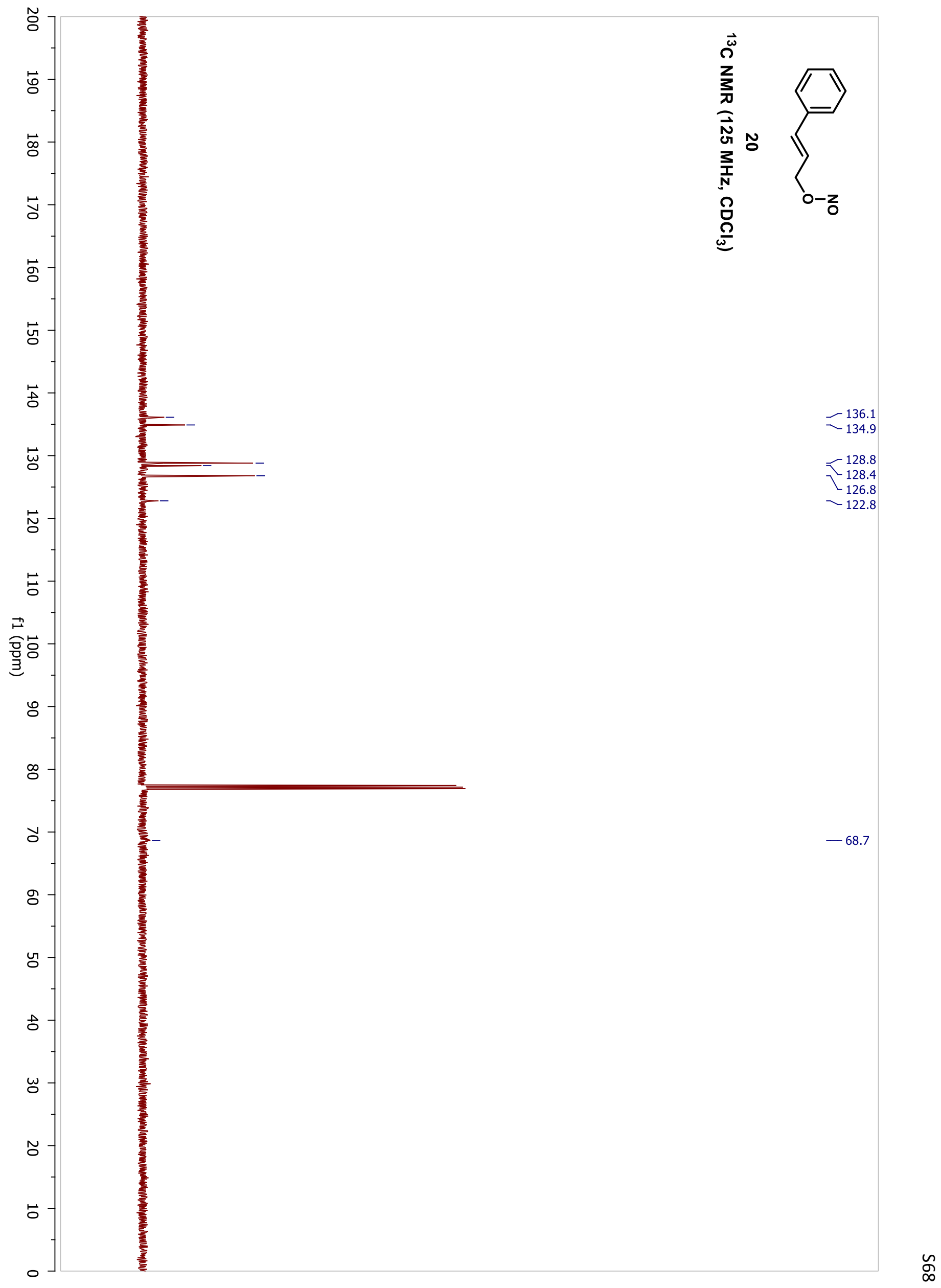




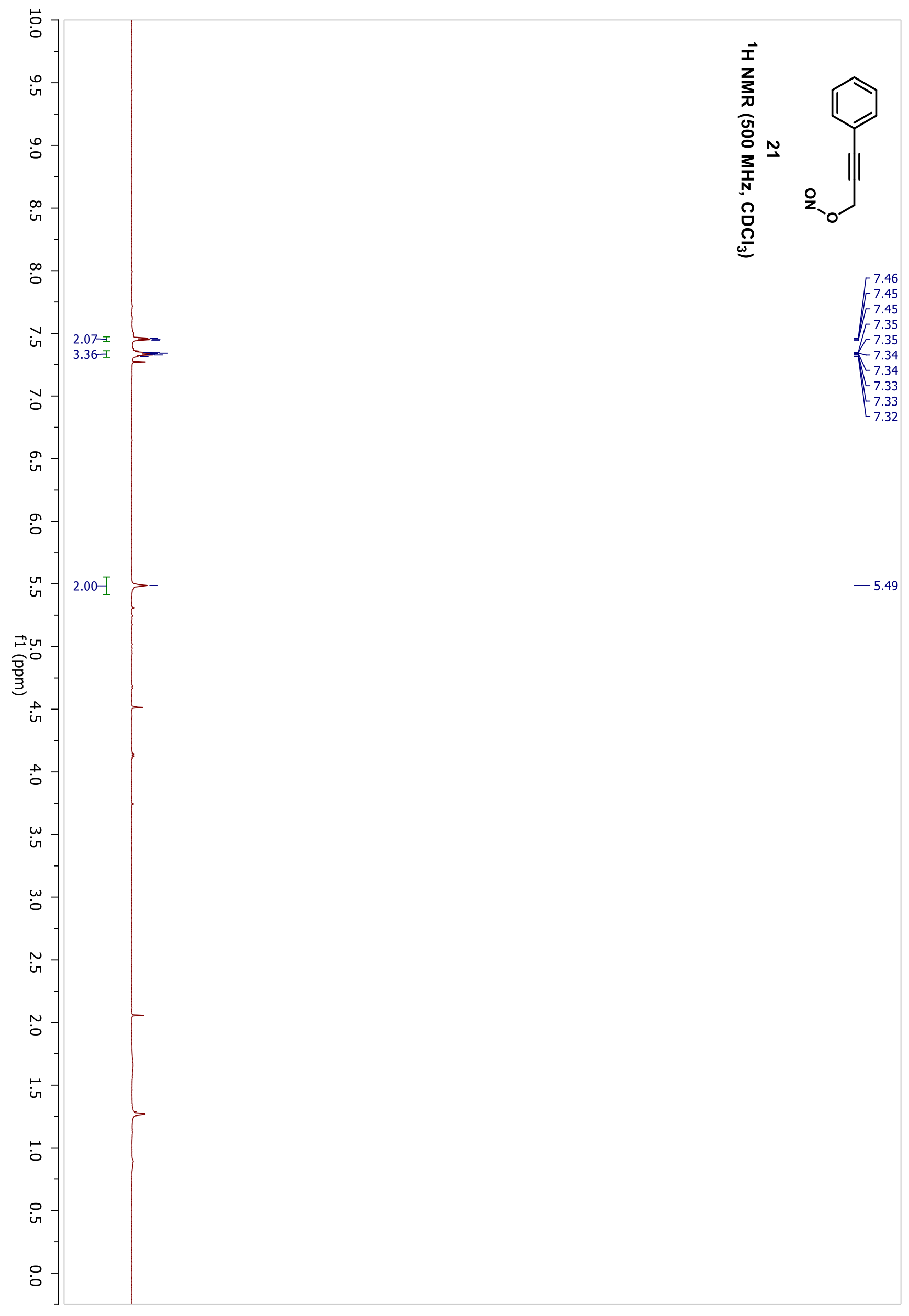




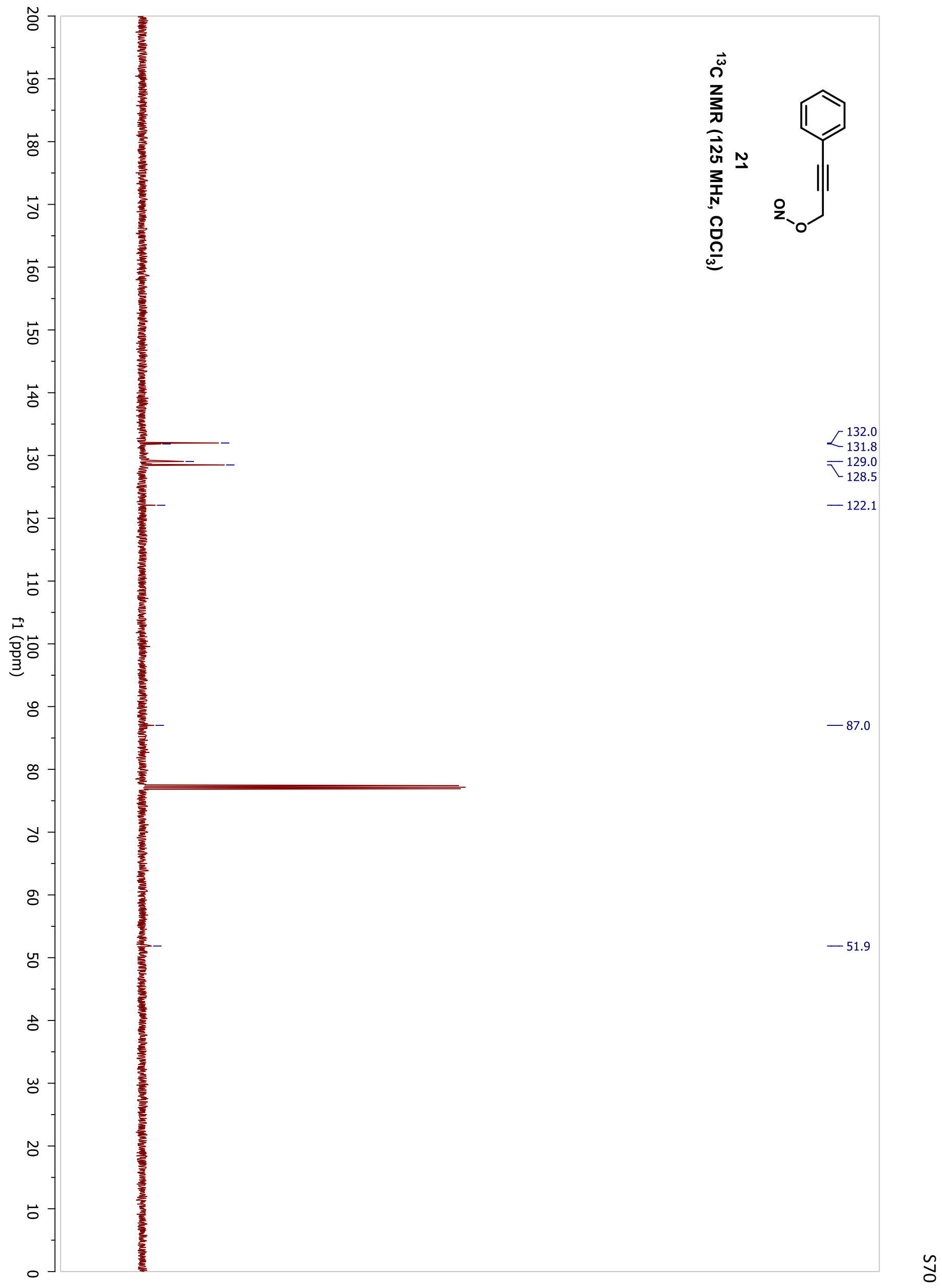




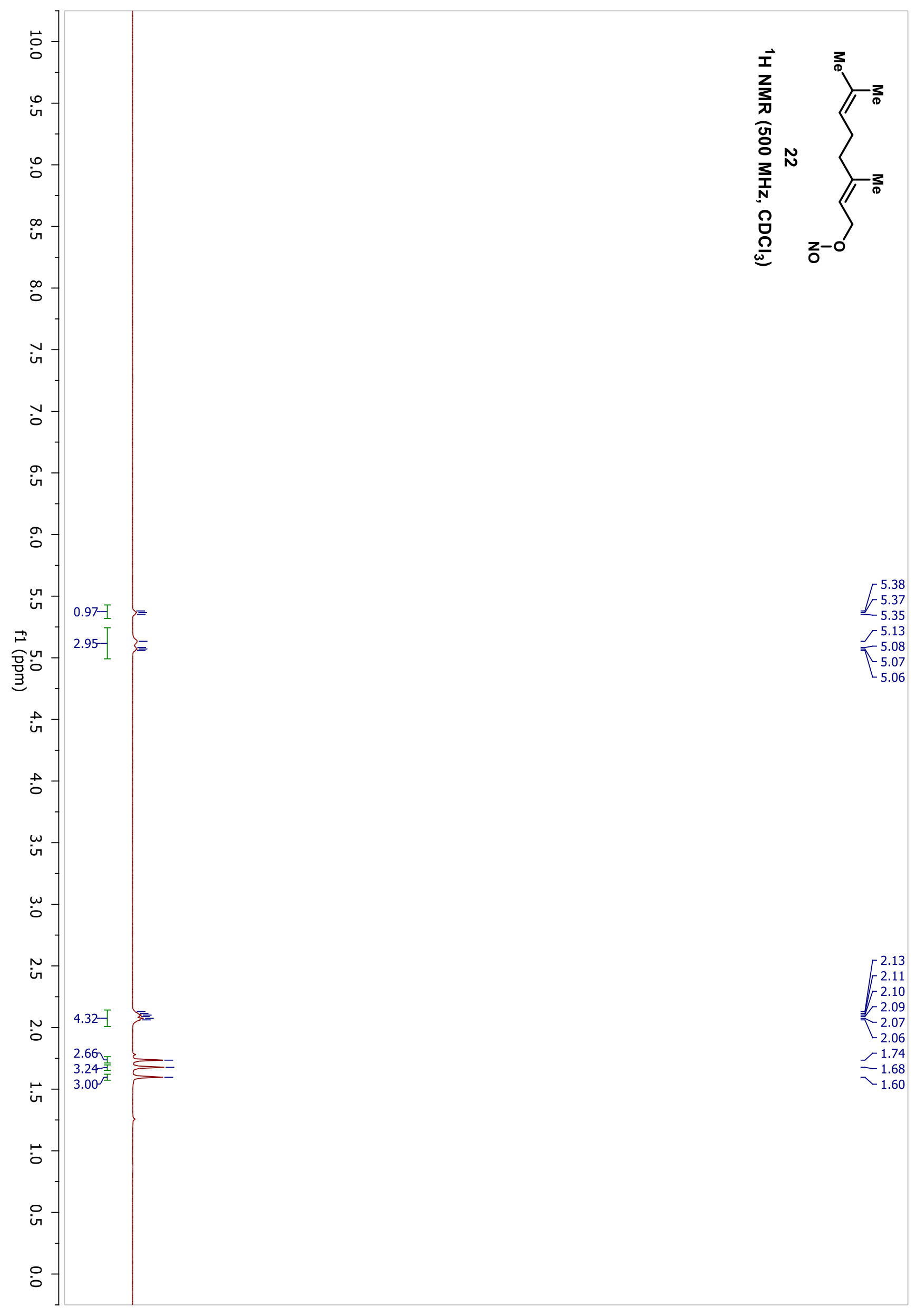




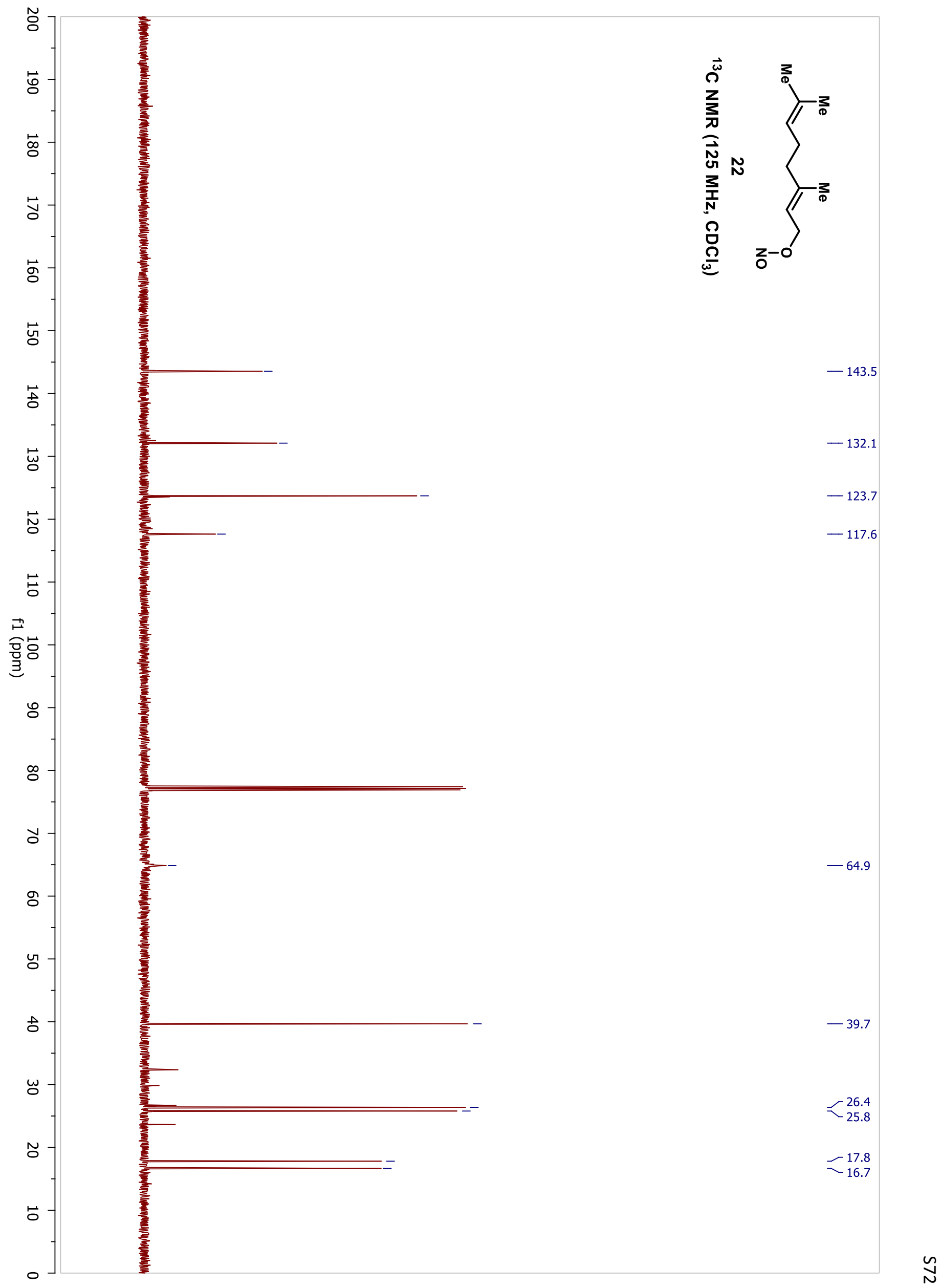




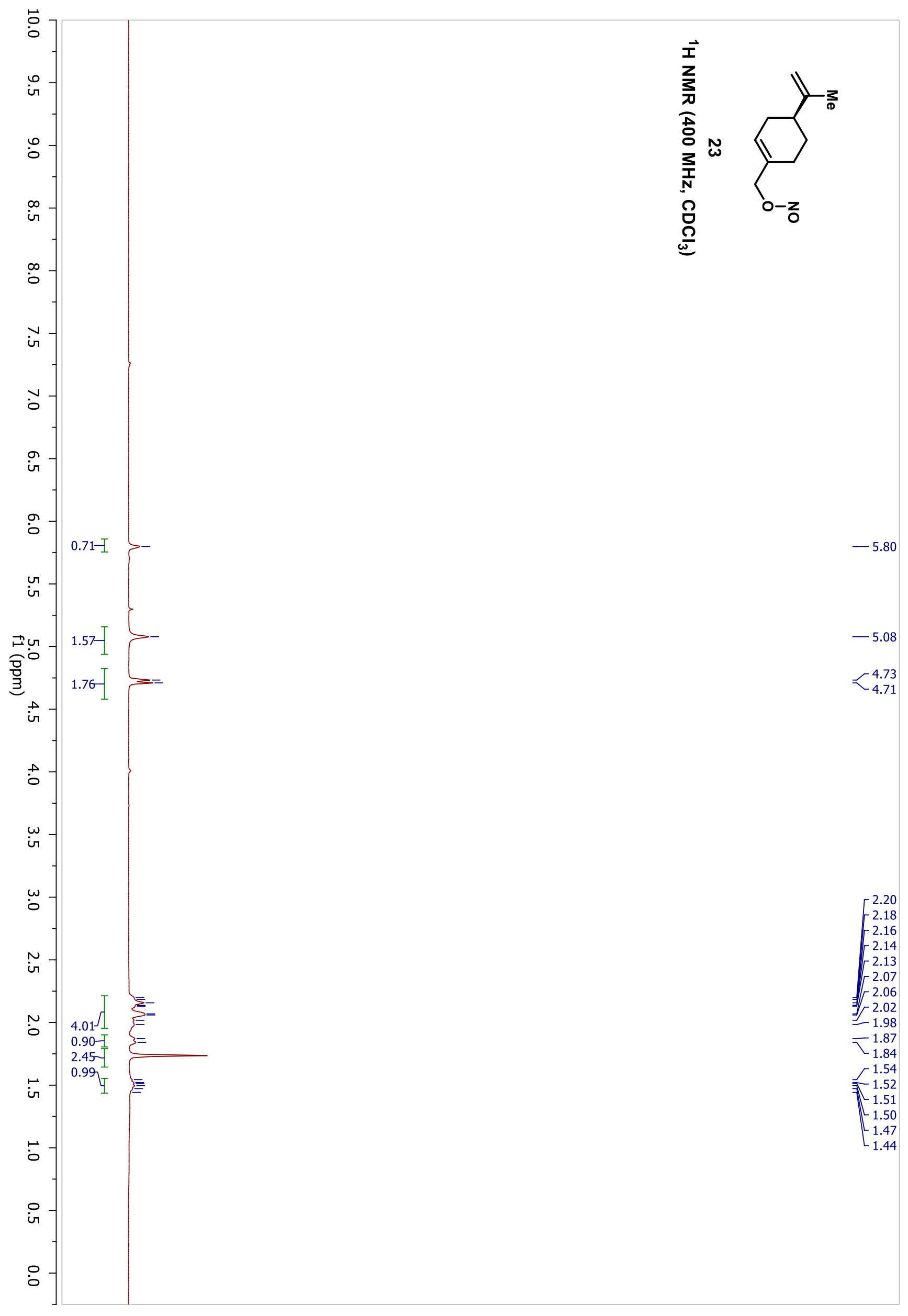




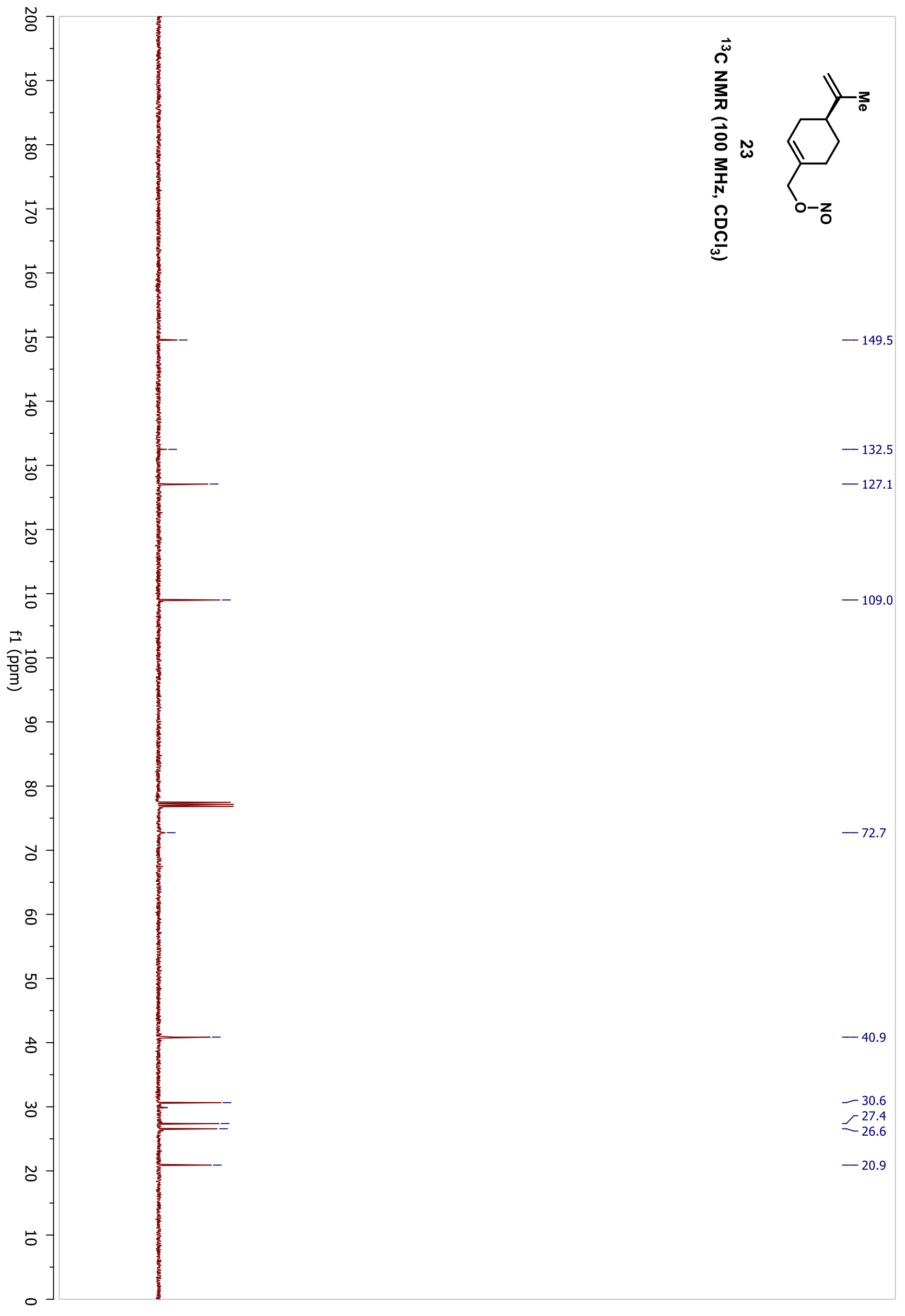

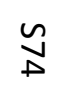




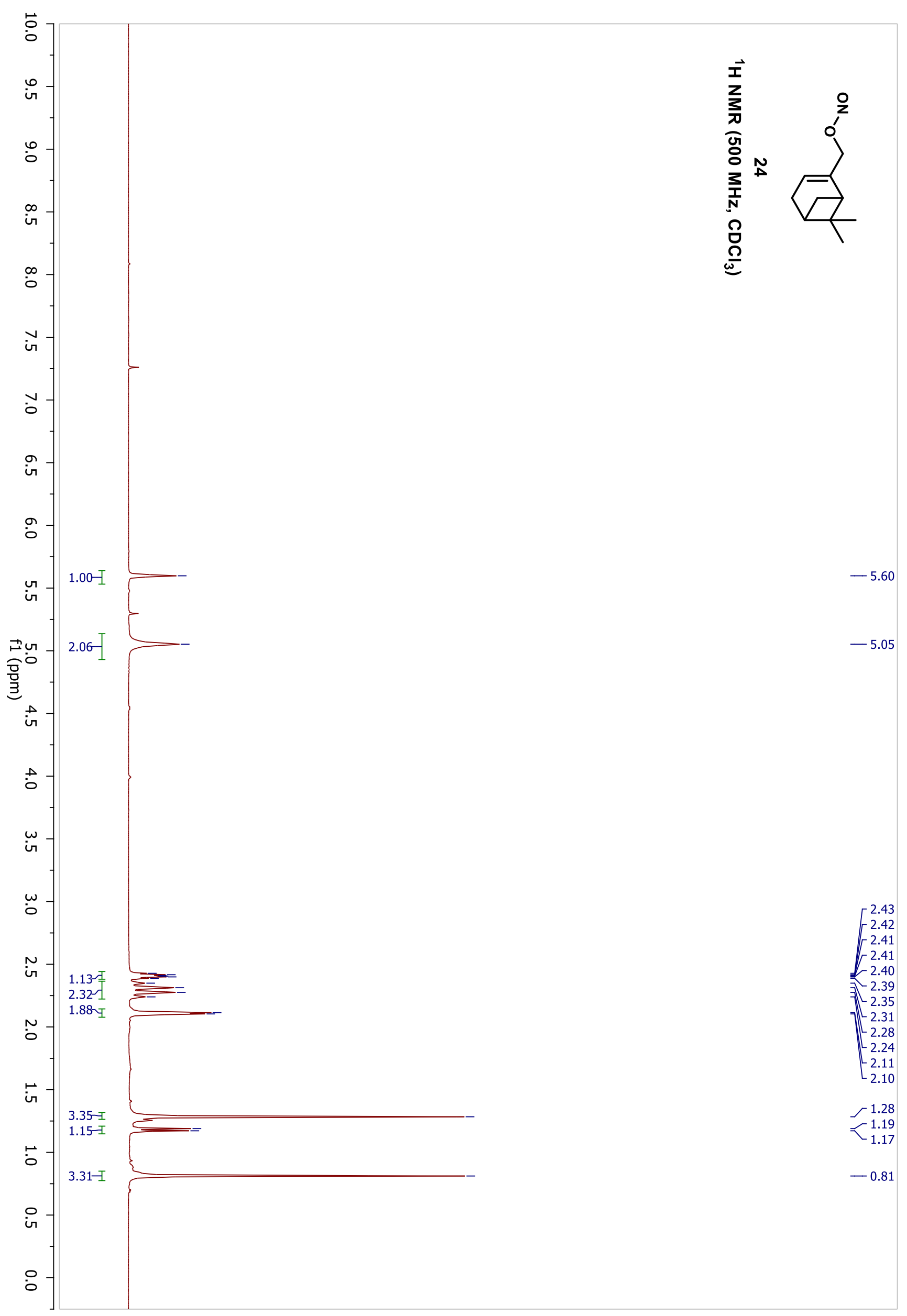




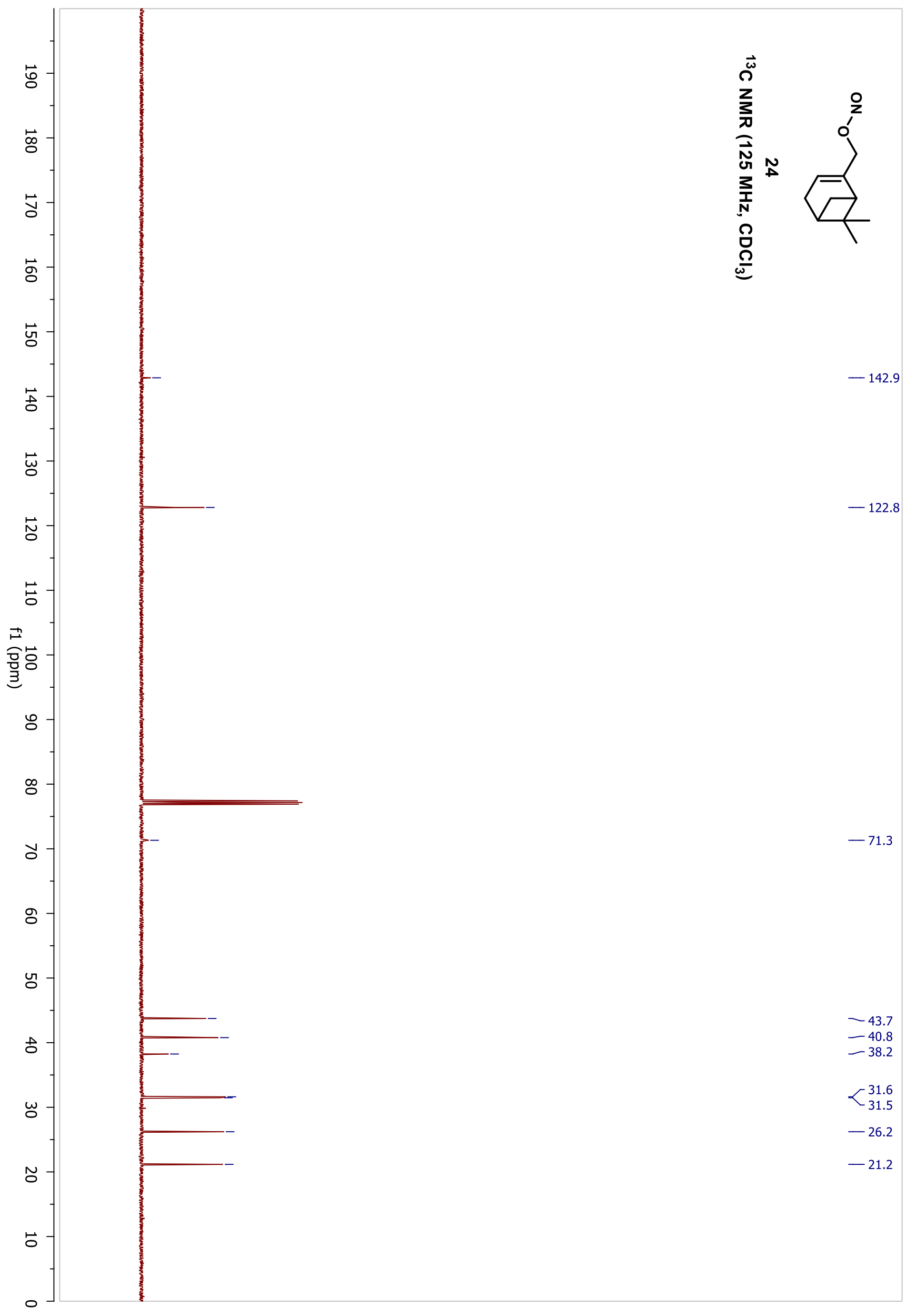

$\tilde{\sigma}$ 


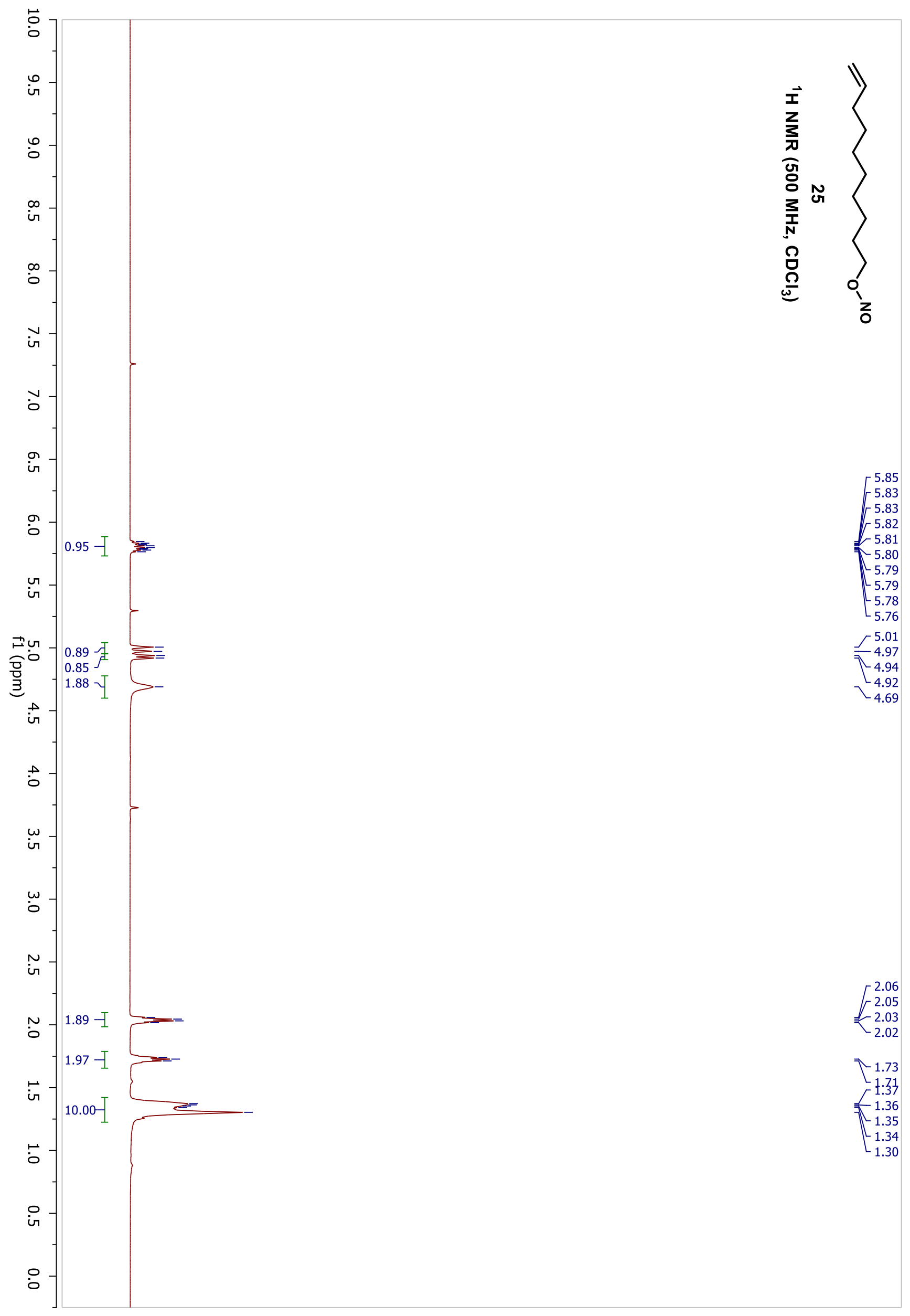




$$
\text { E }
$$




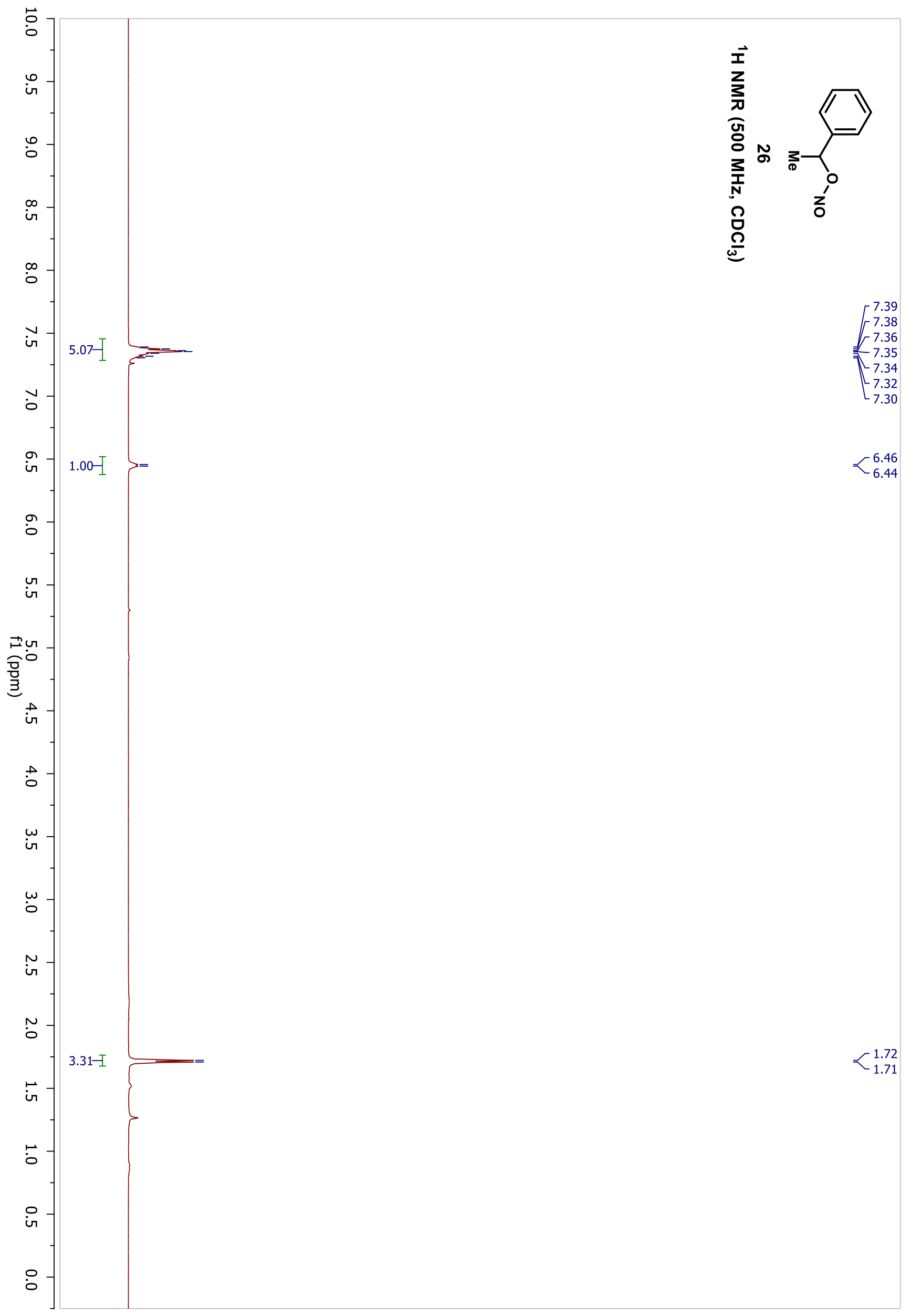




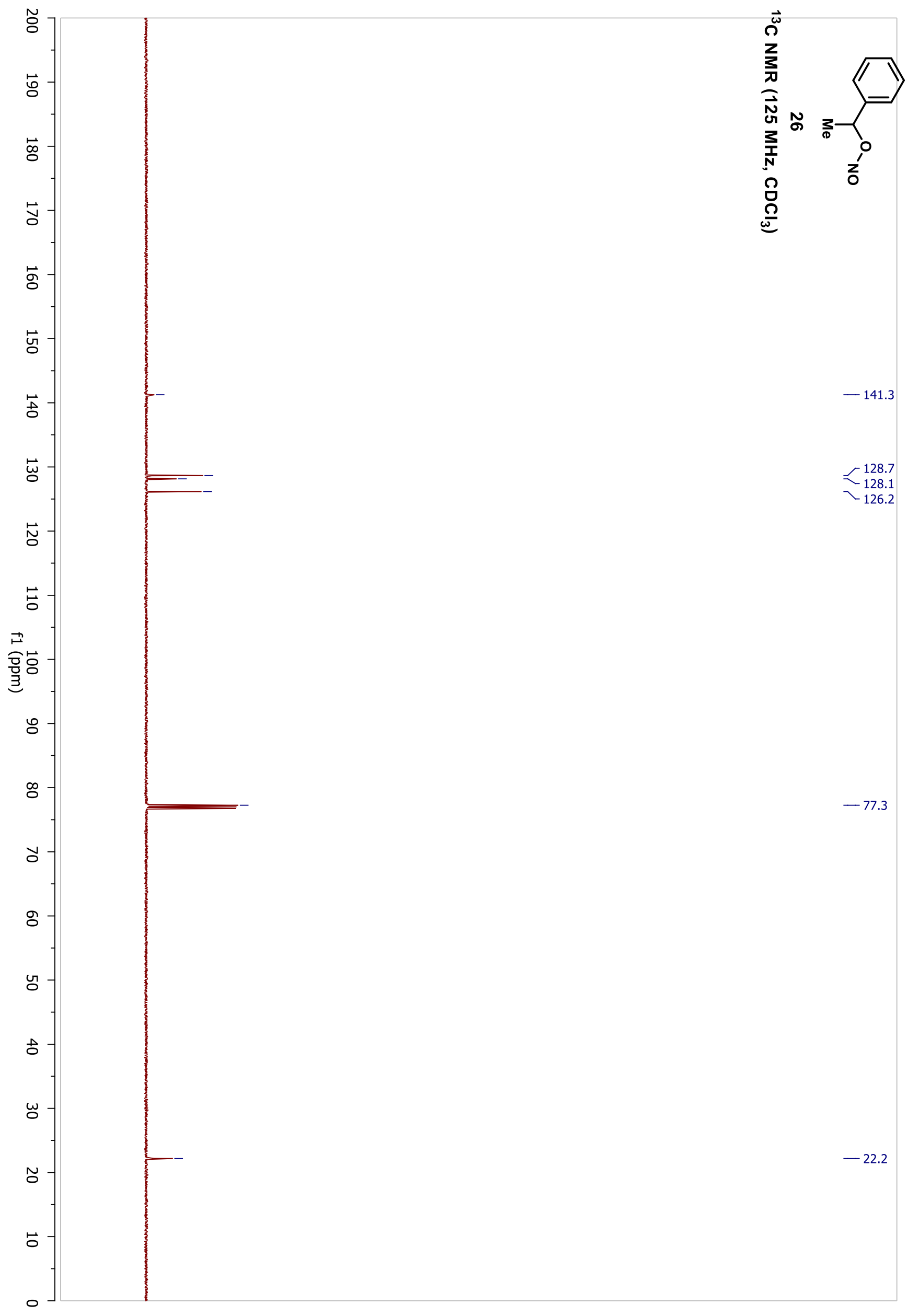




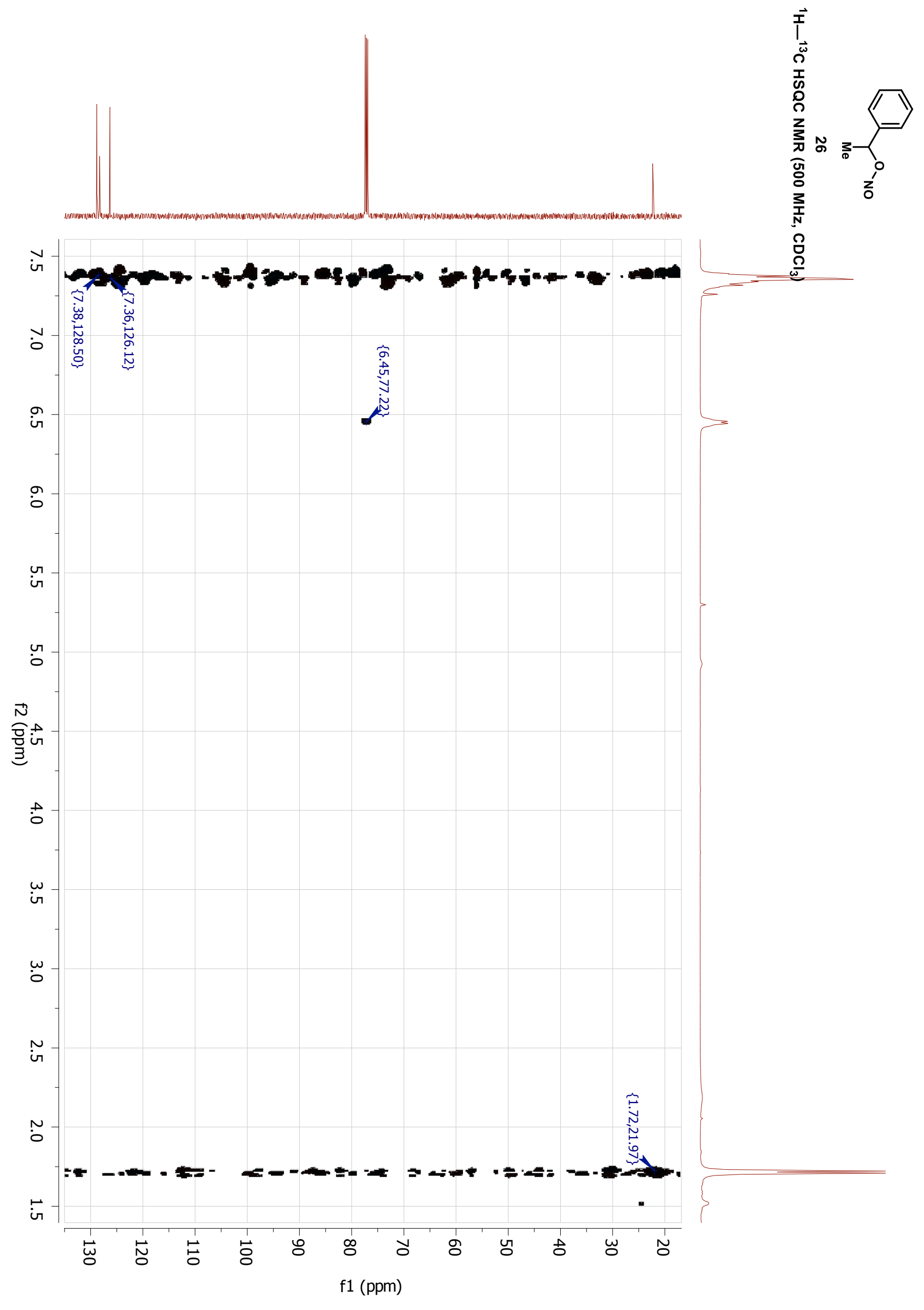




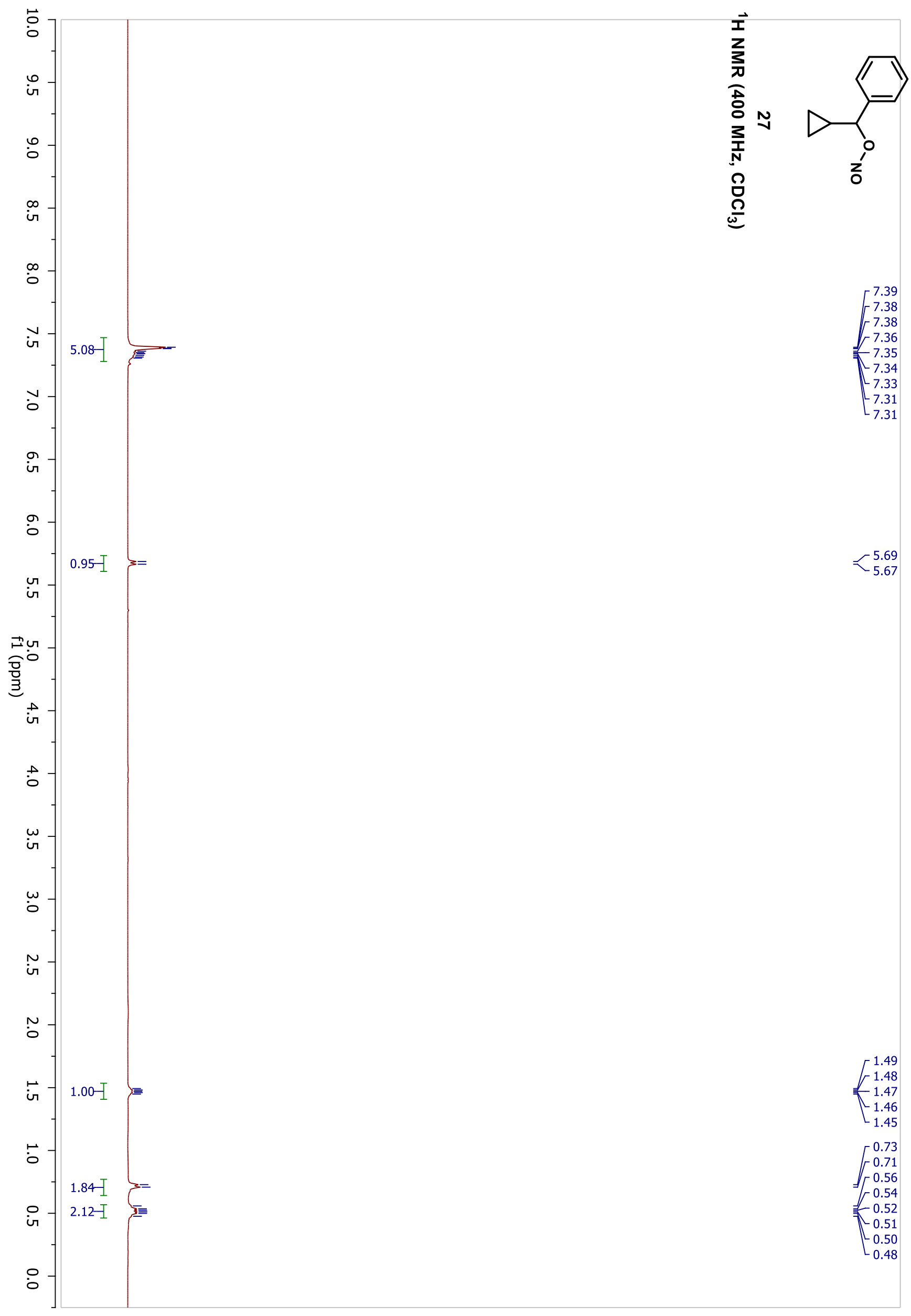




$$
\left.\right|^{n+2}
$$




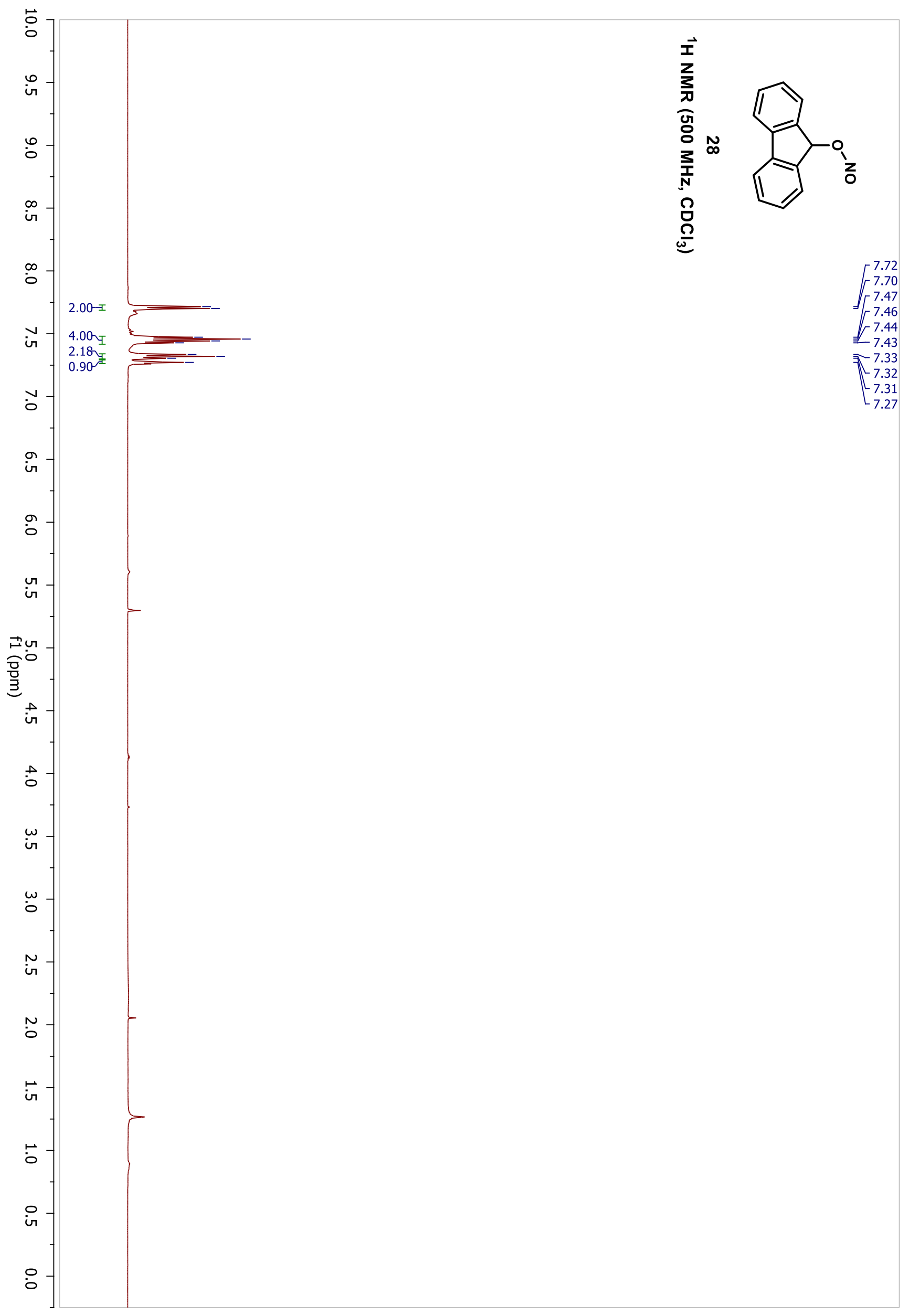




$$
E^{\prime \prime}
$$




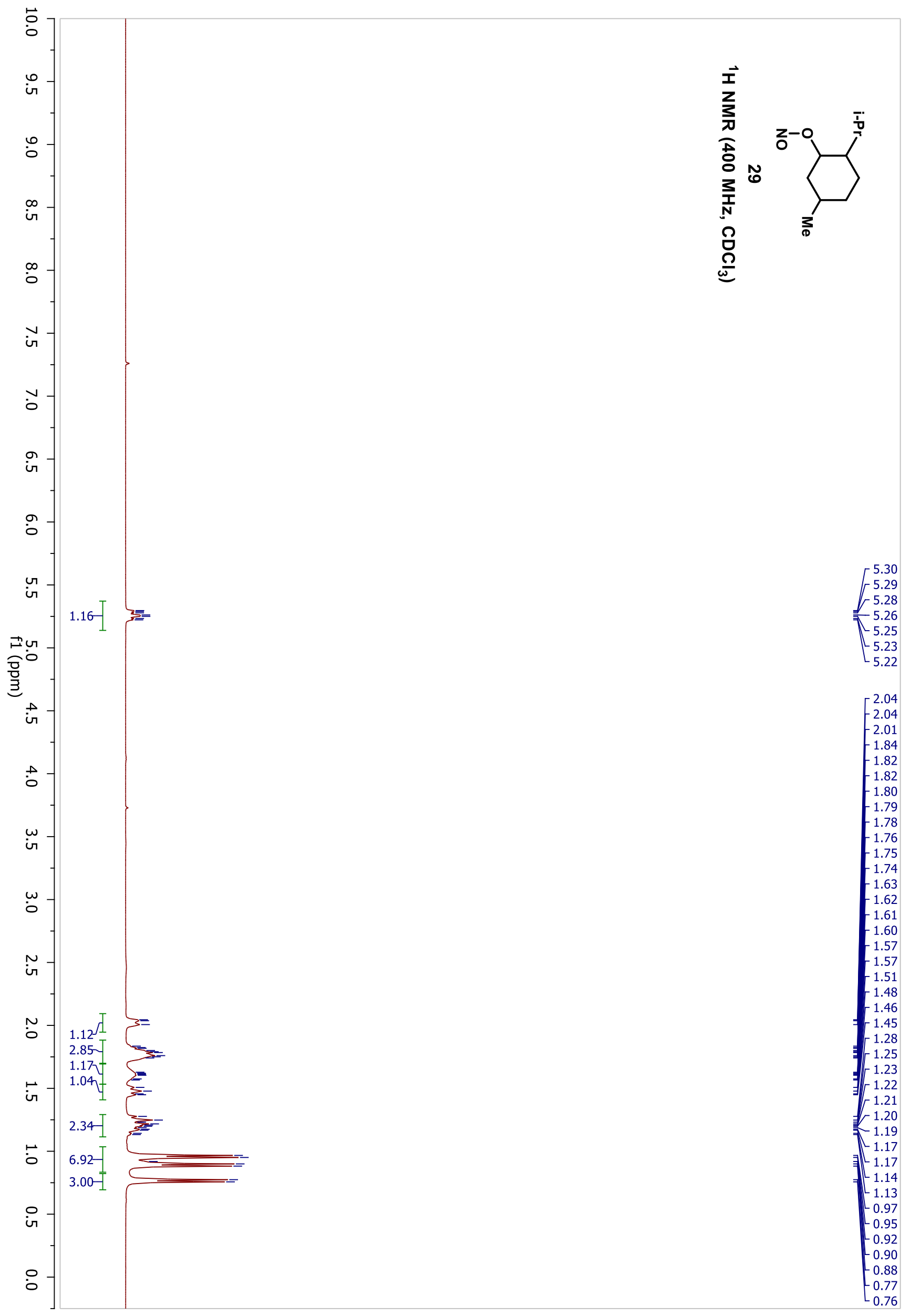




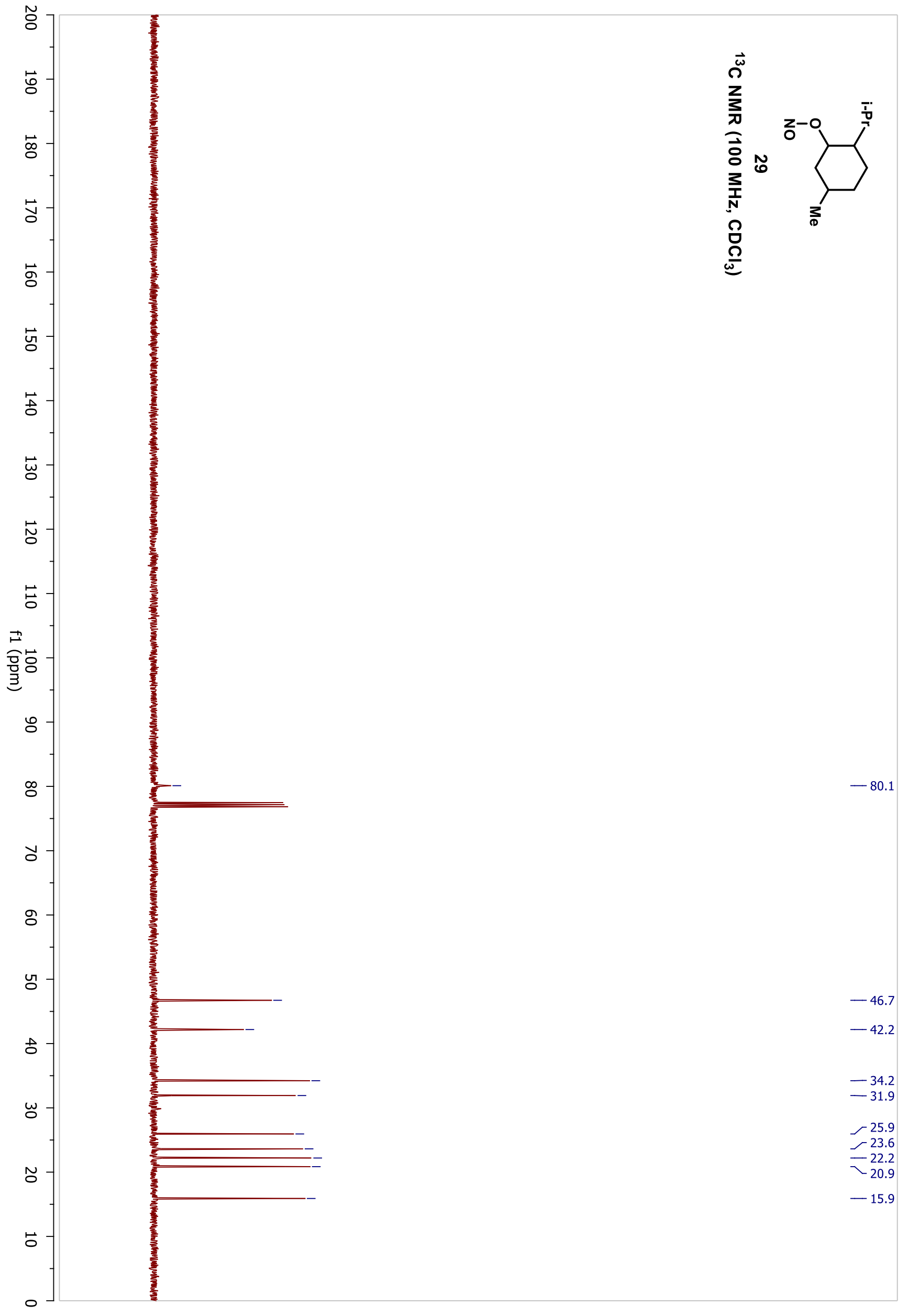




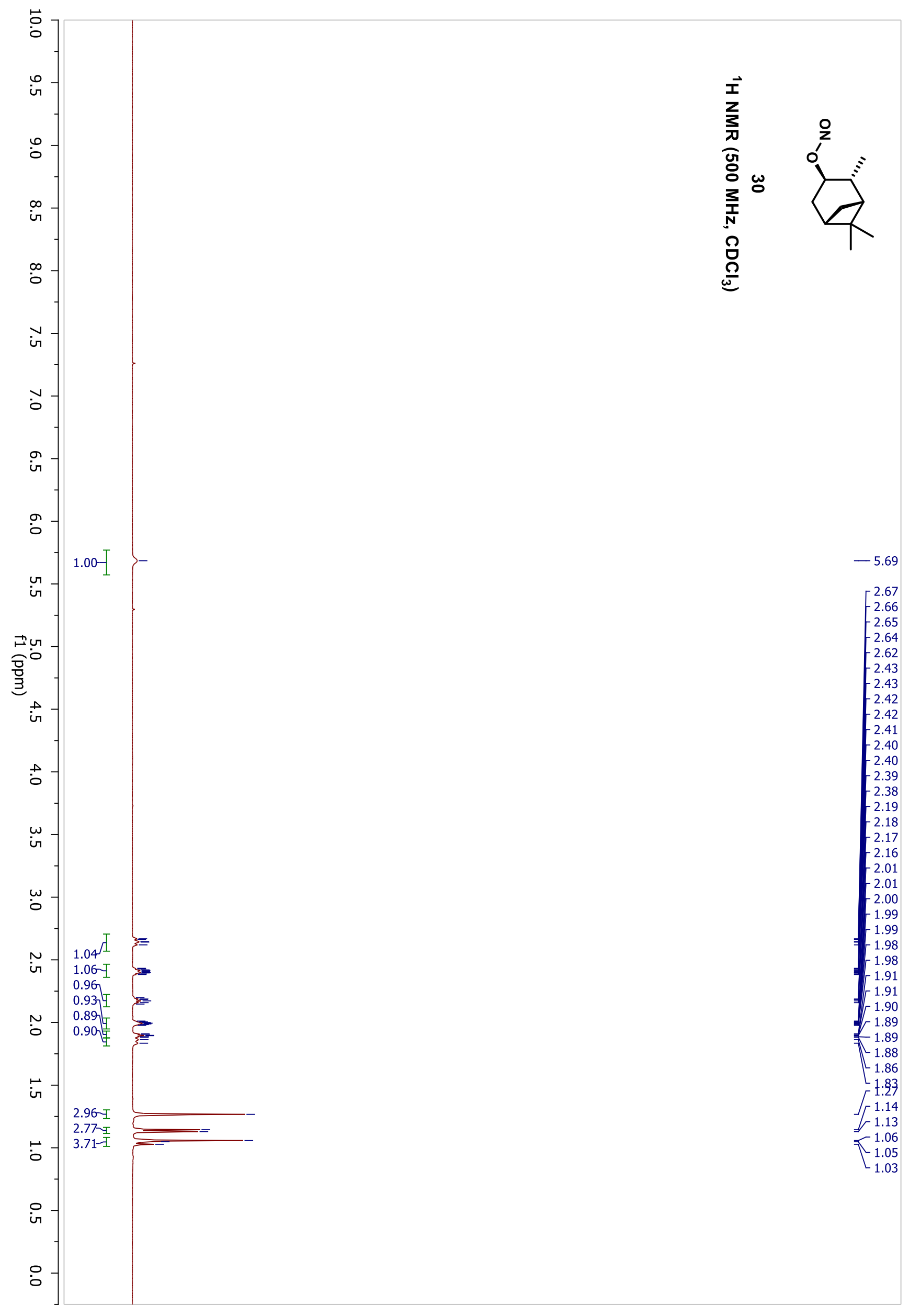




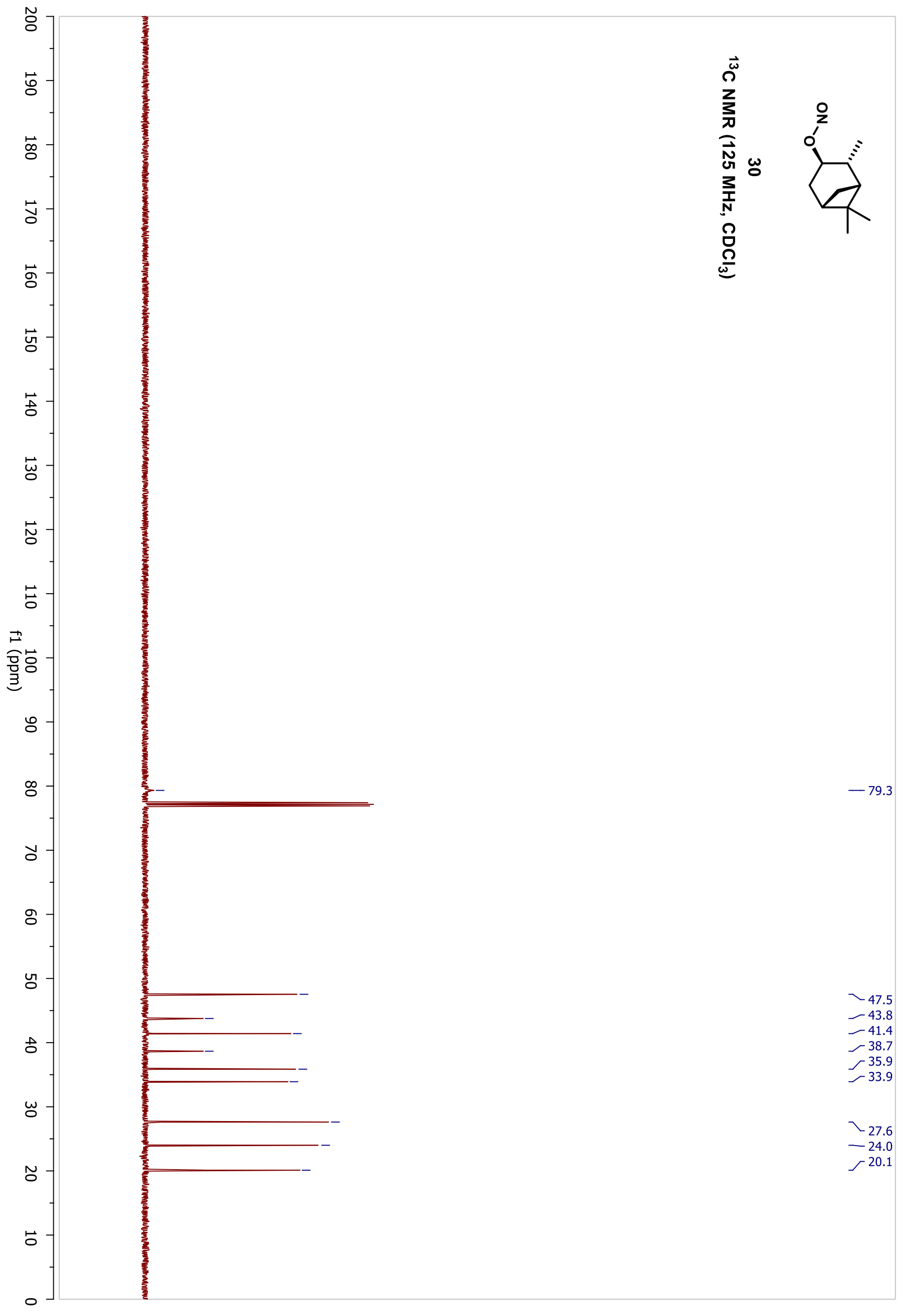




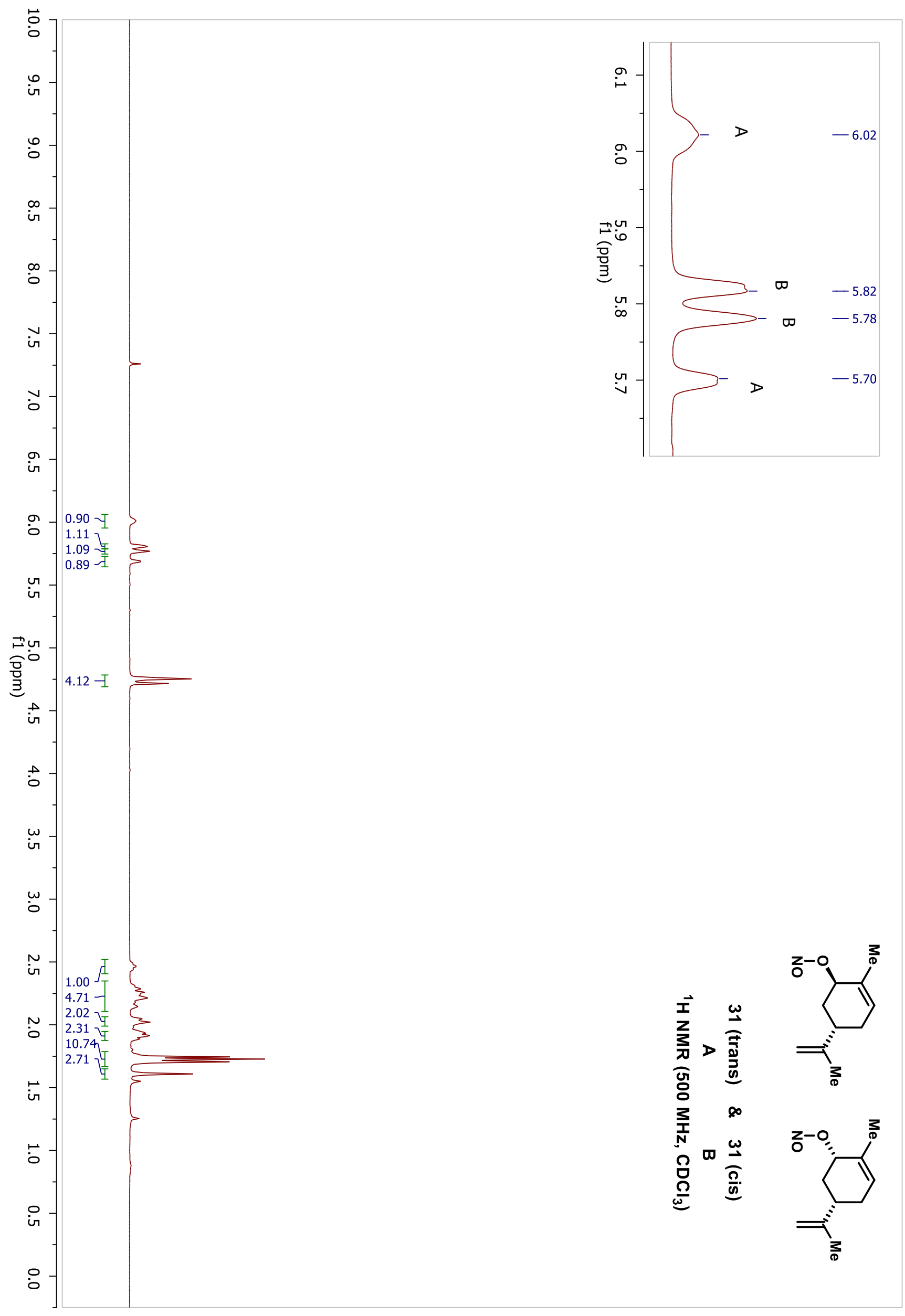




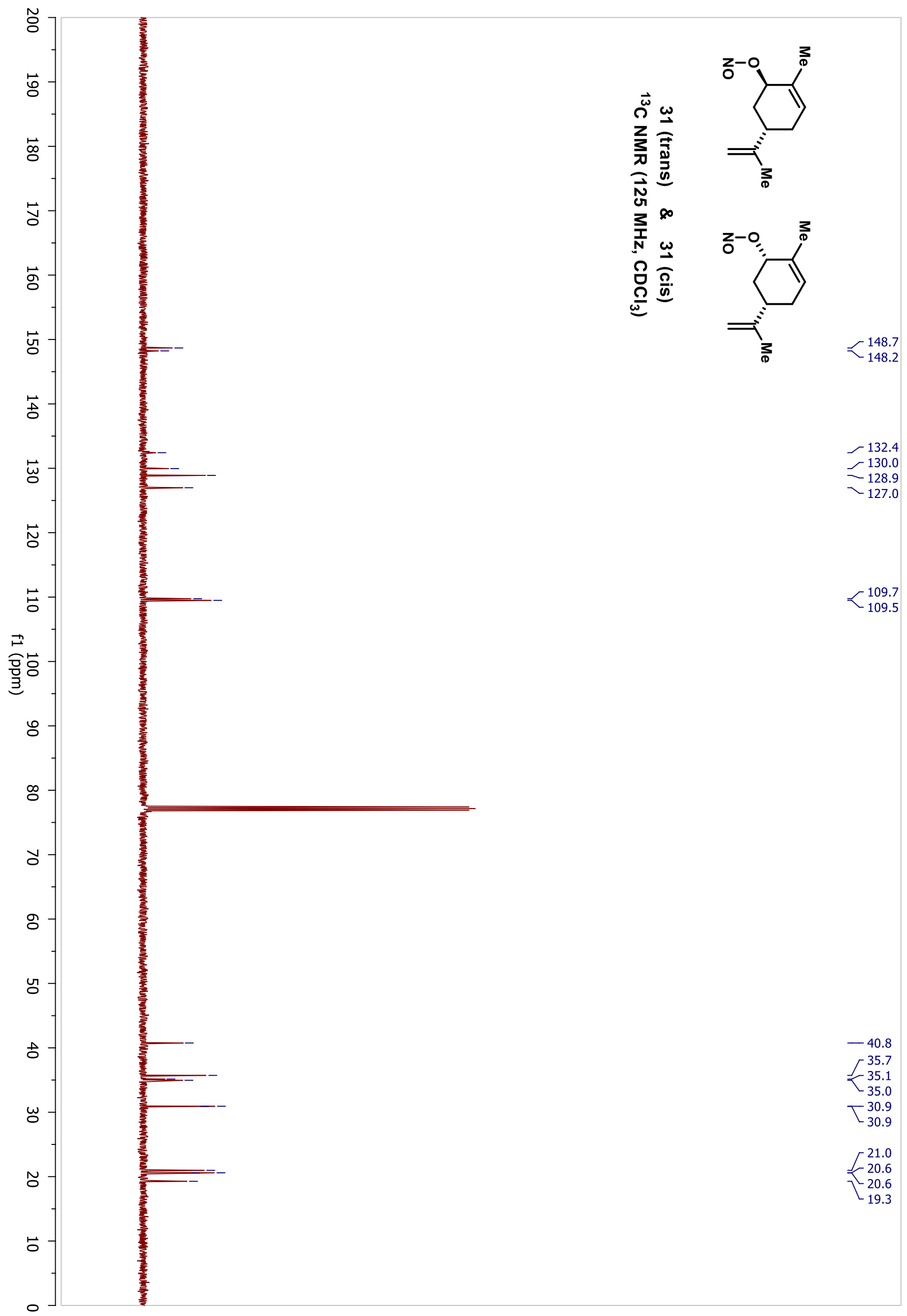

$\mathscr{\varrho}$ 


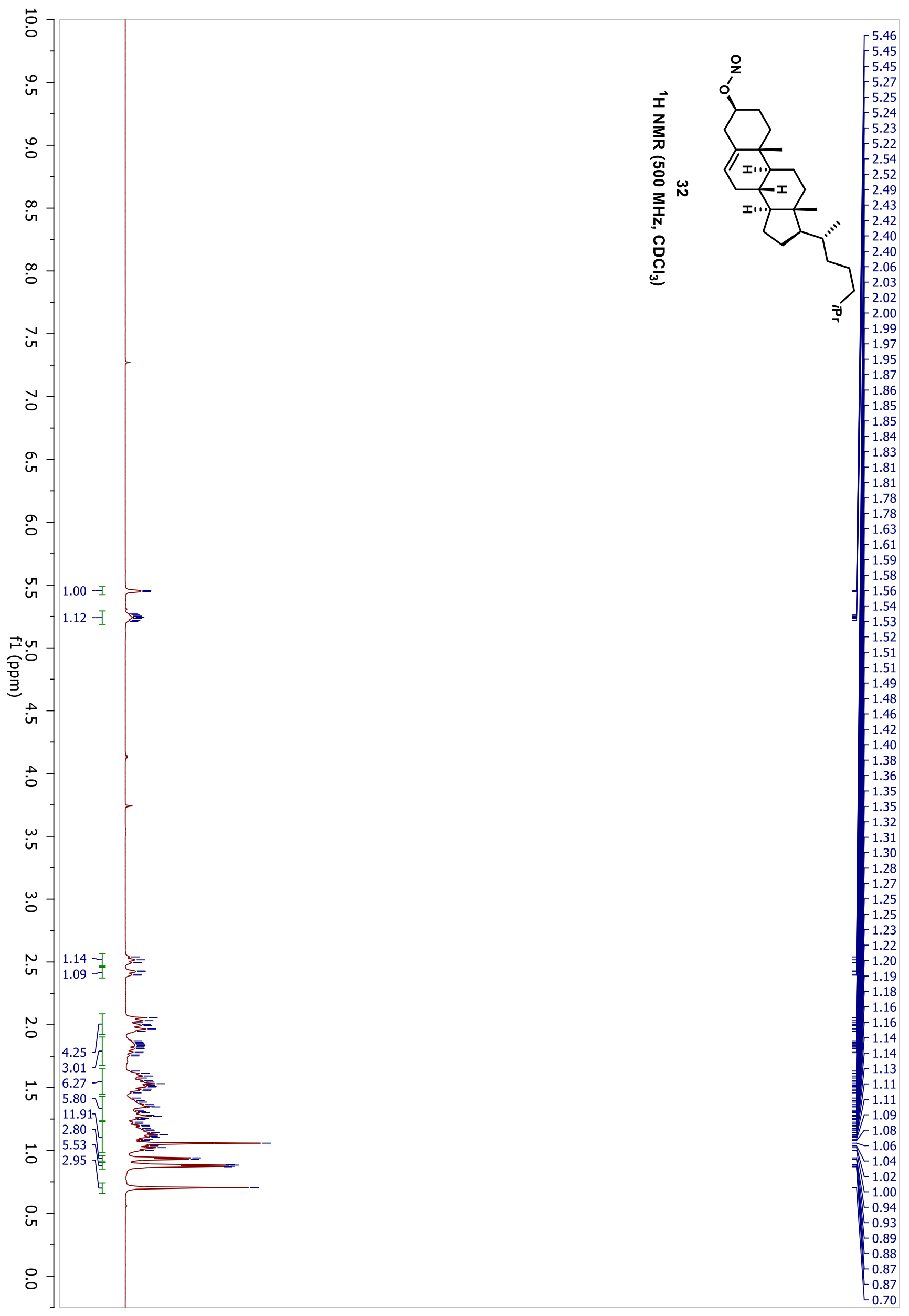




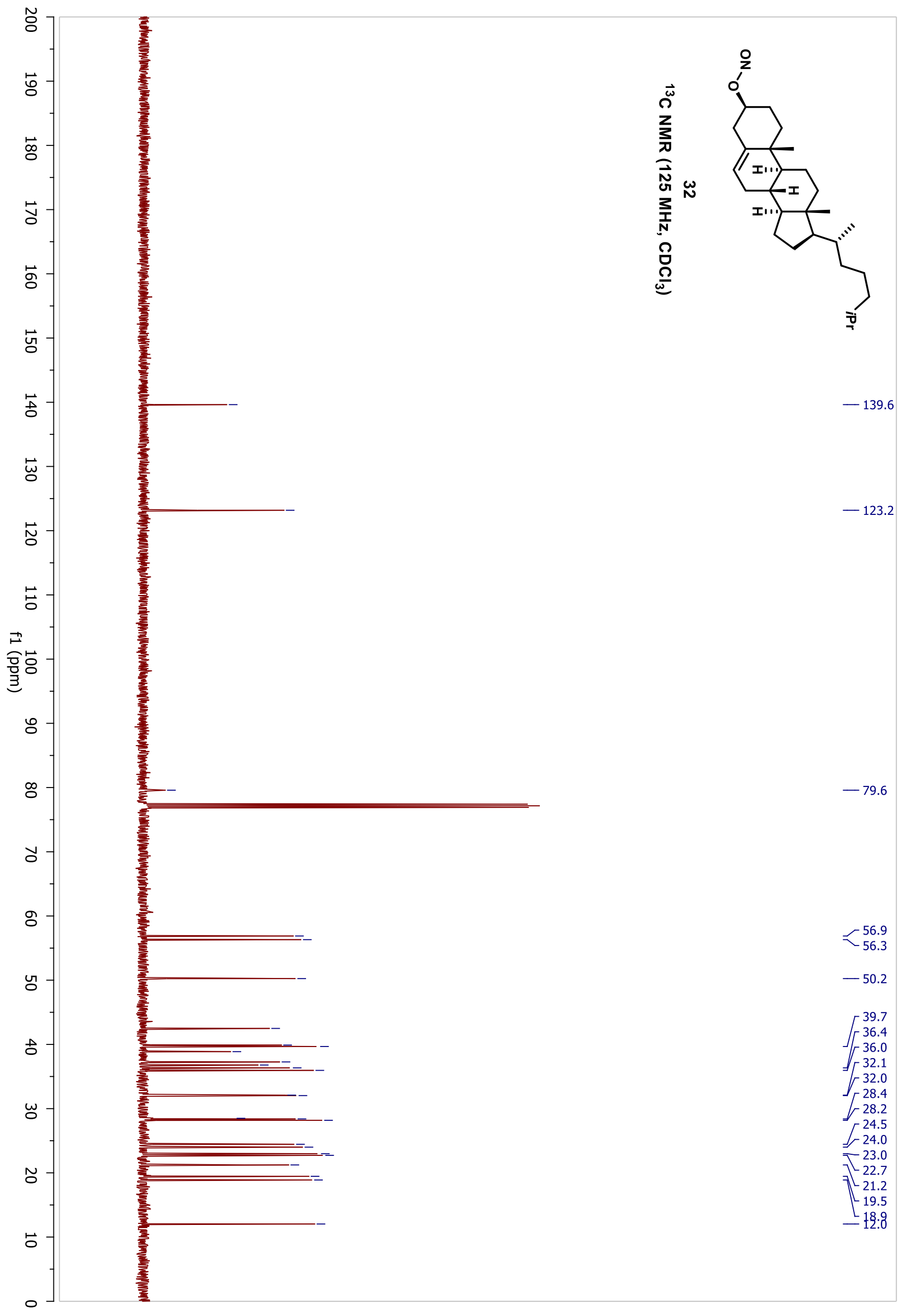




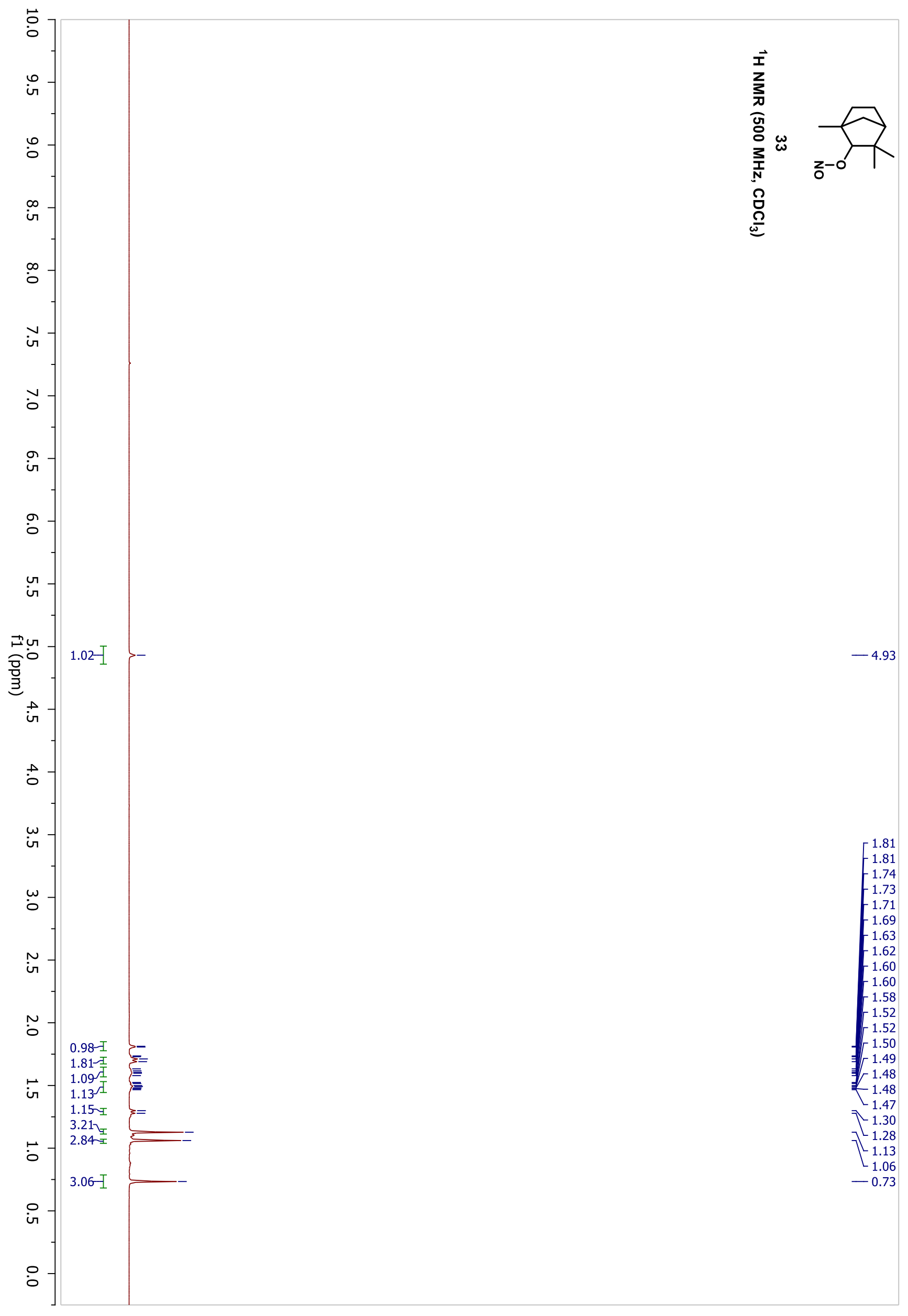




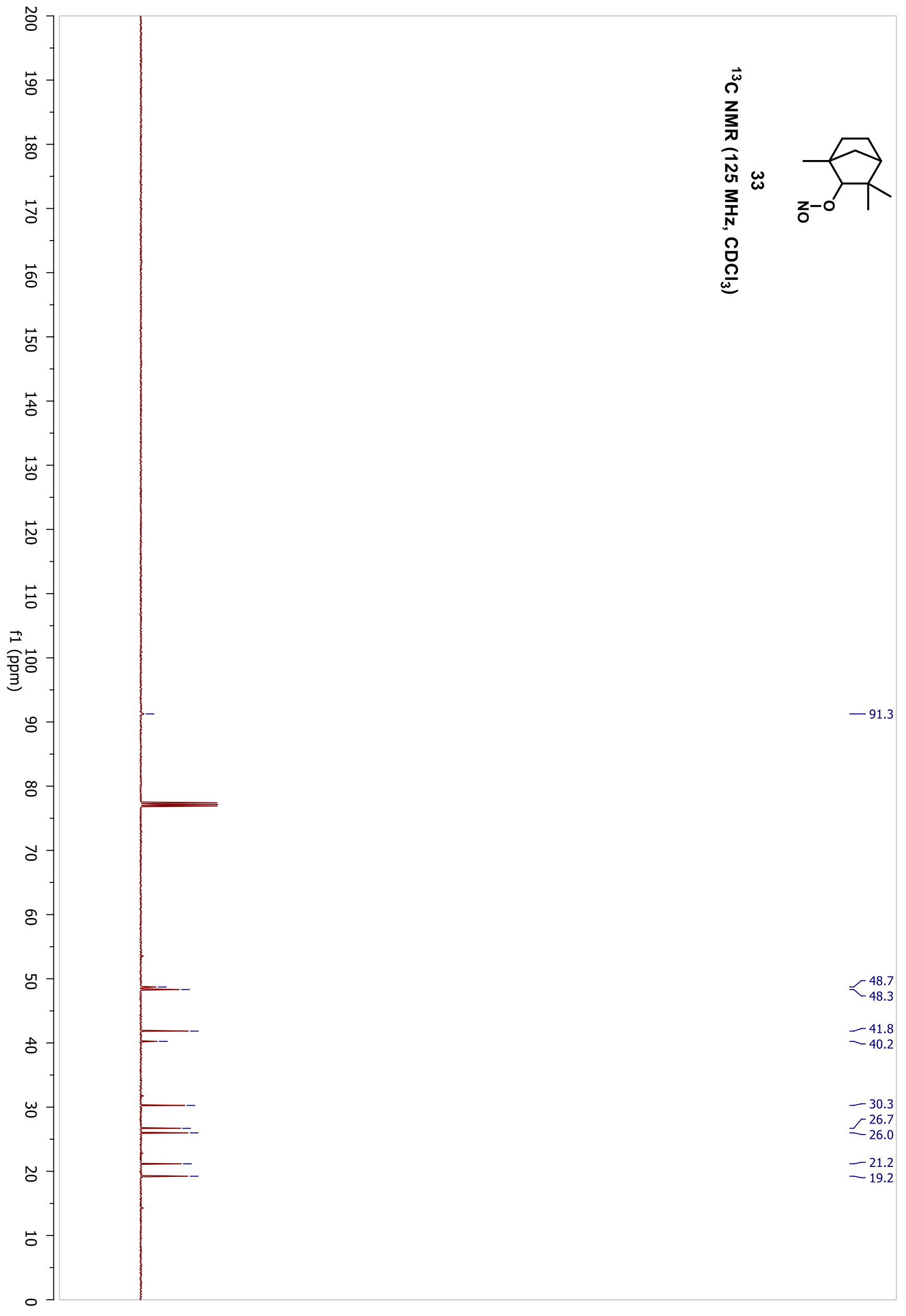




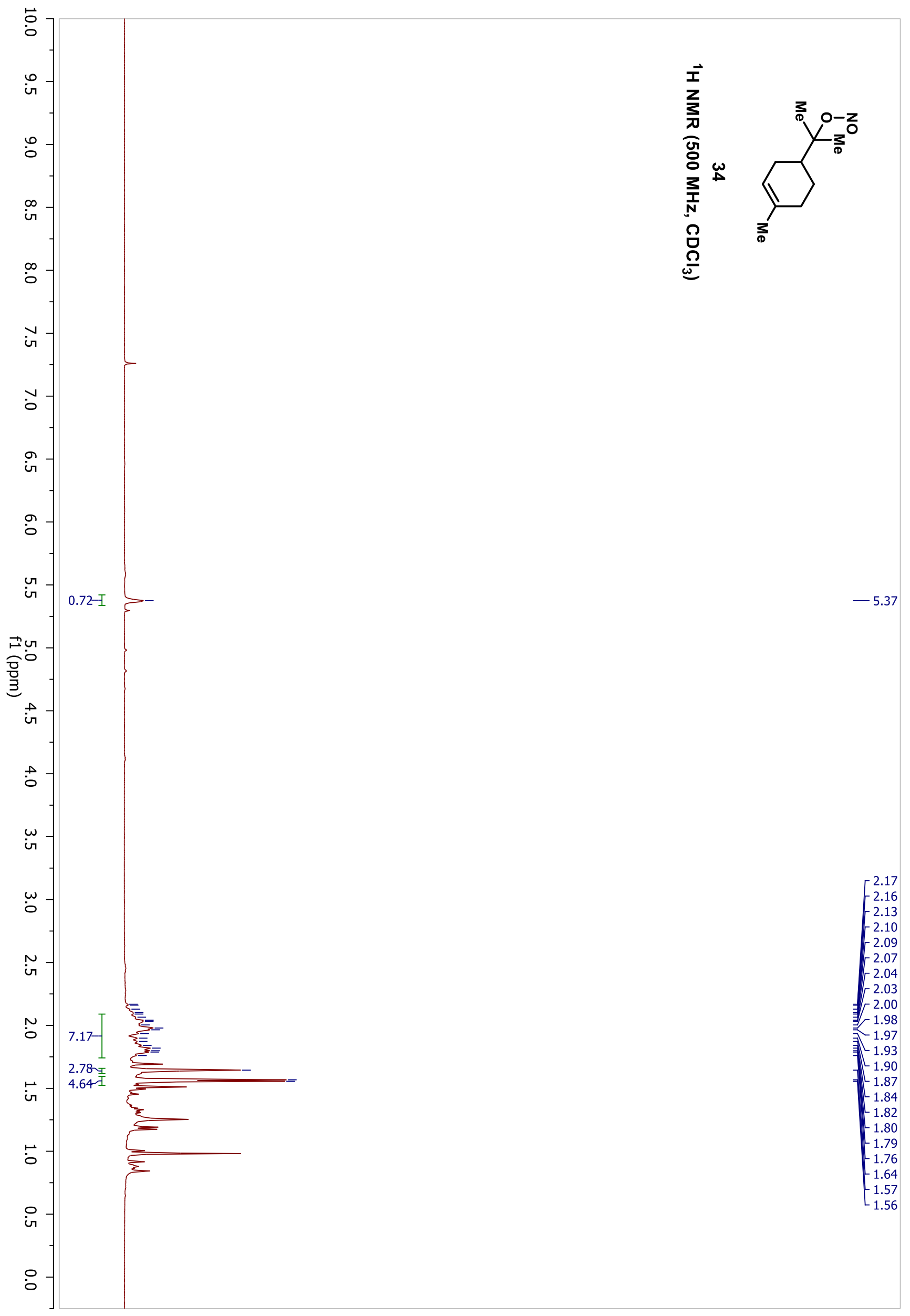




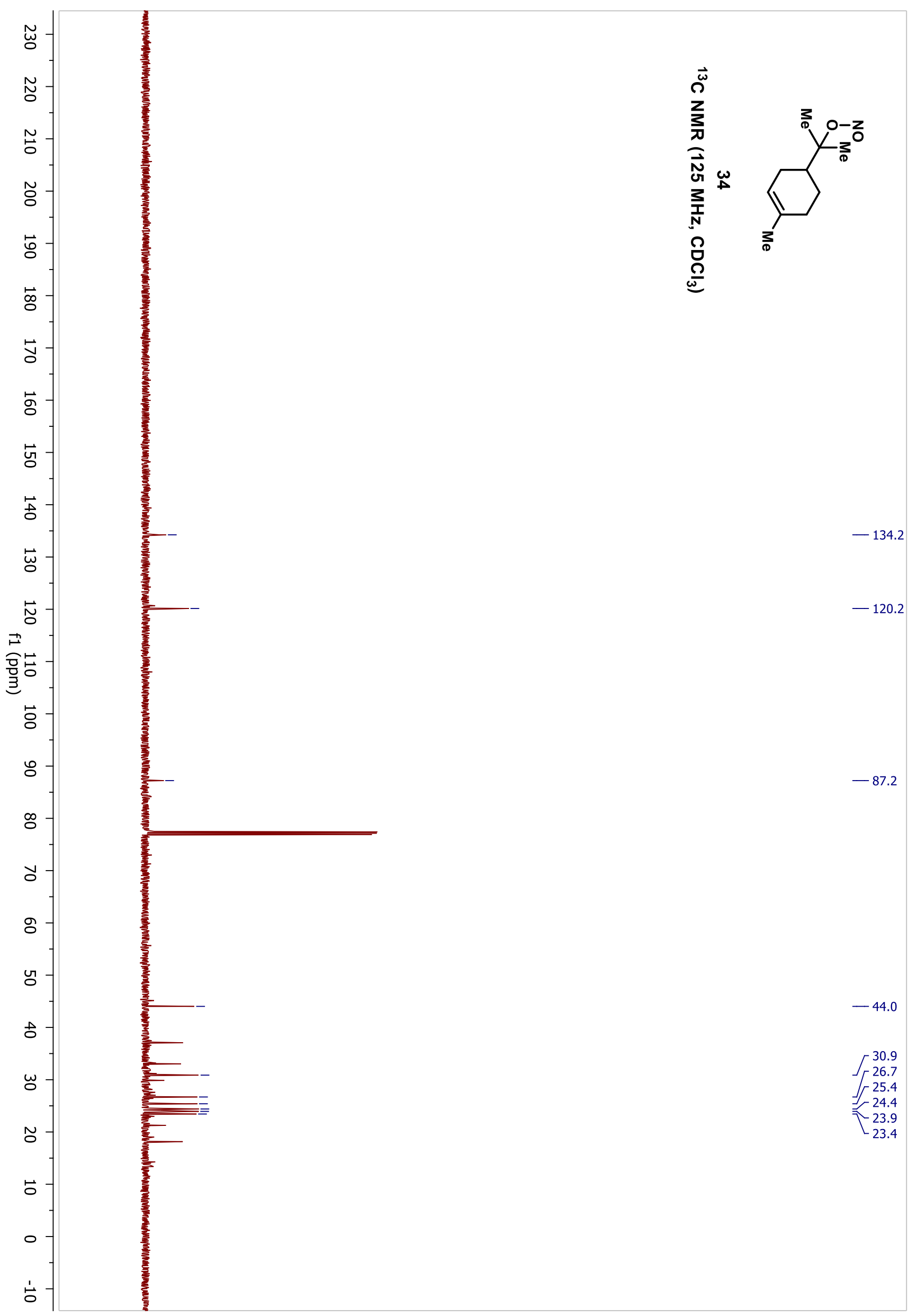




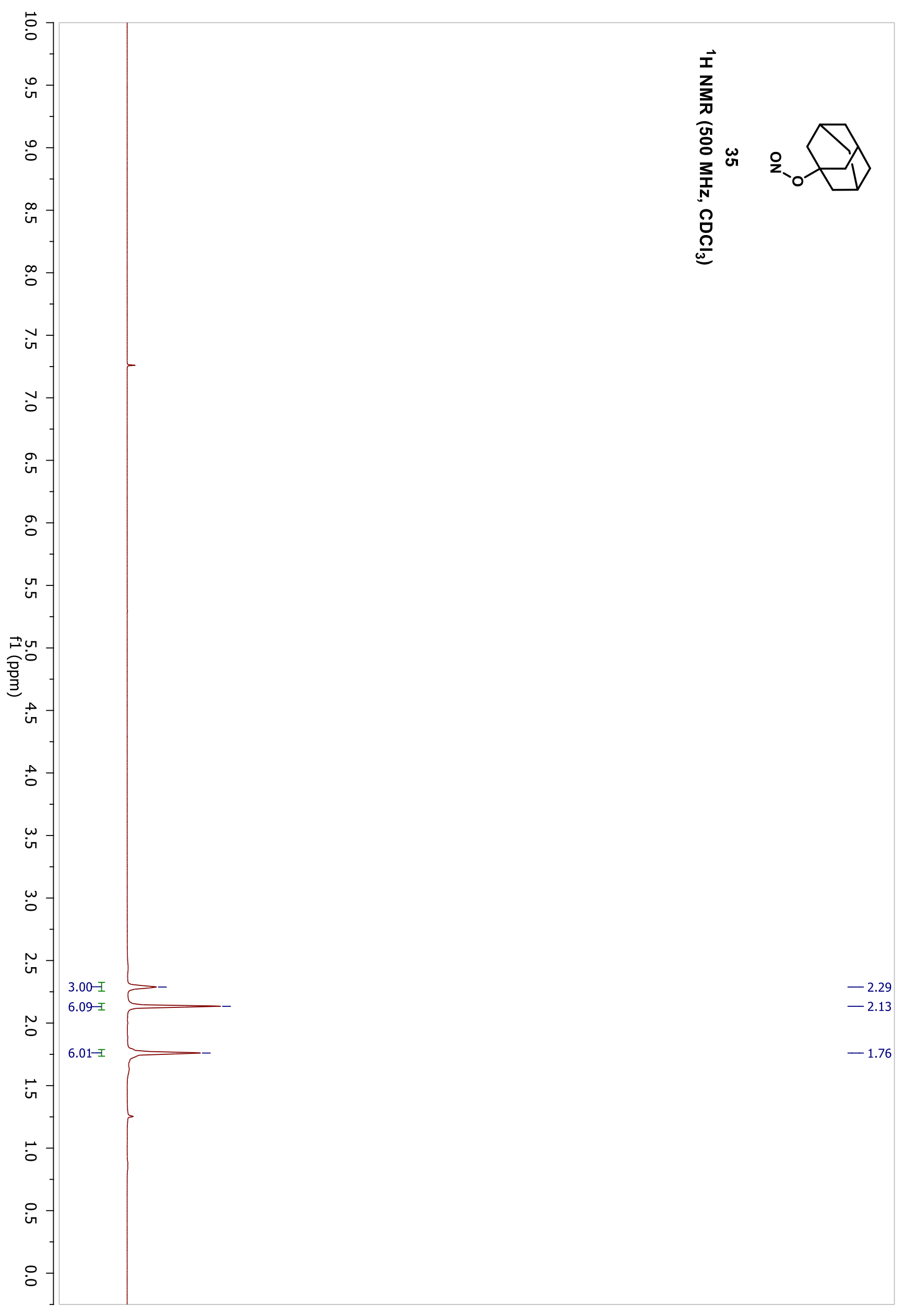




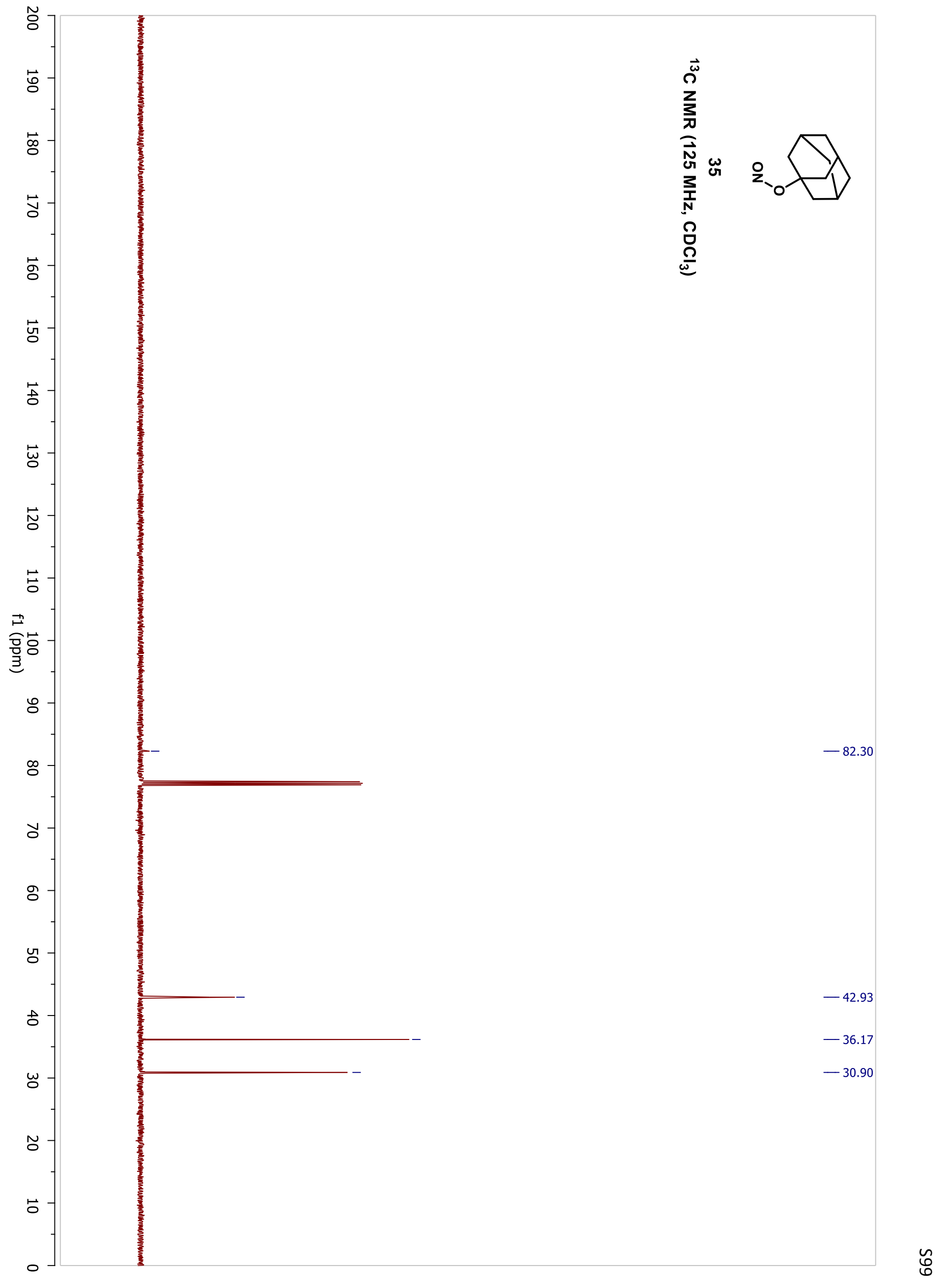




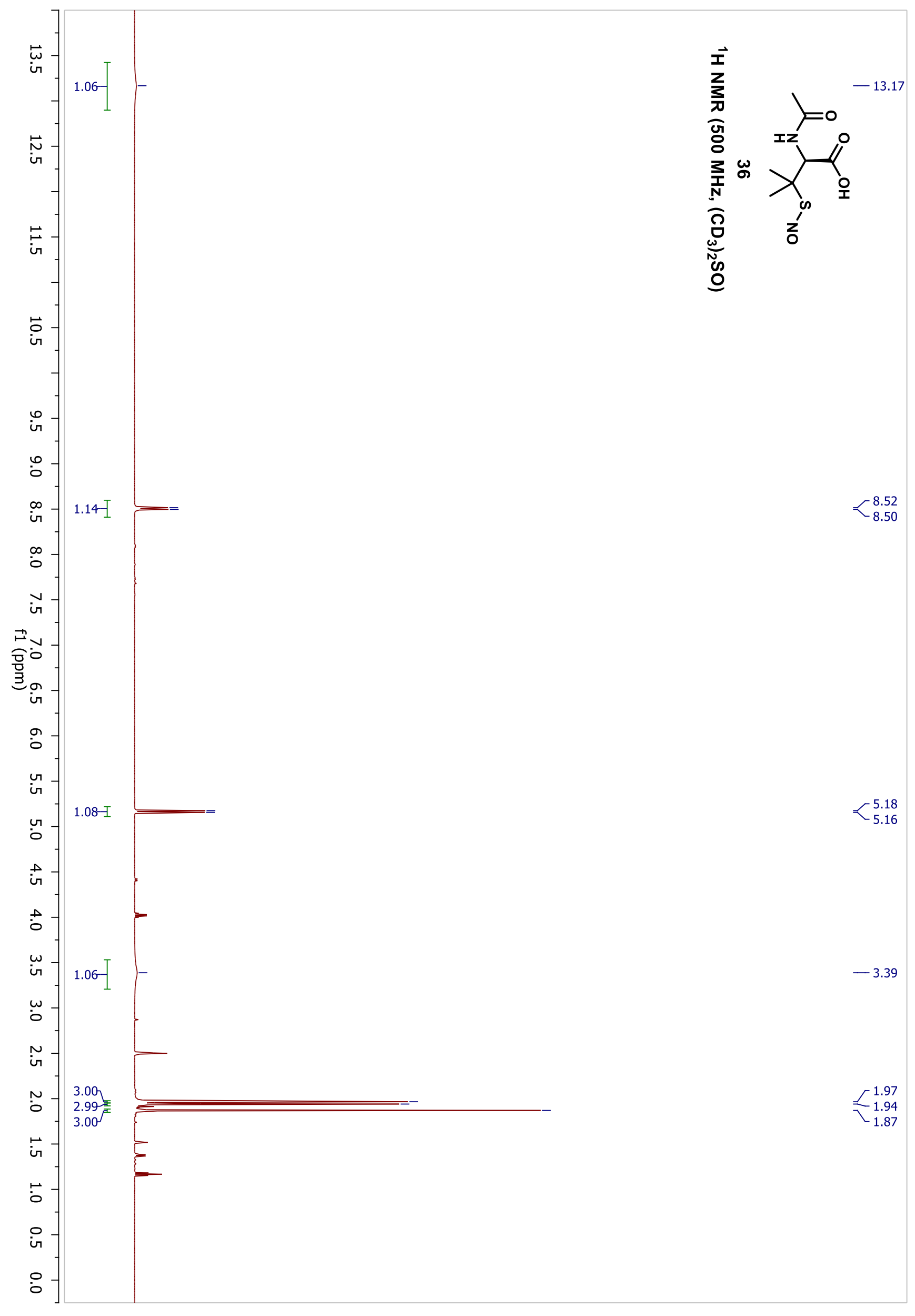

$\check{亏}$ 


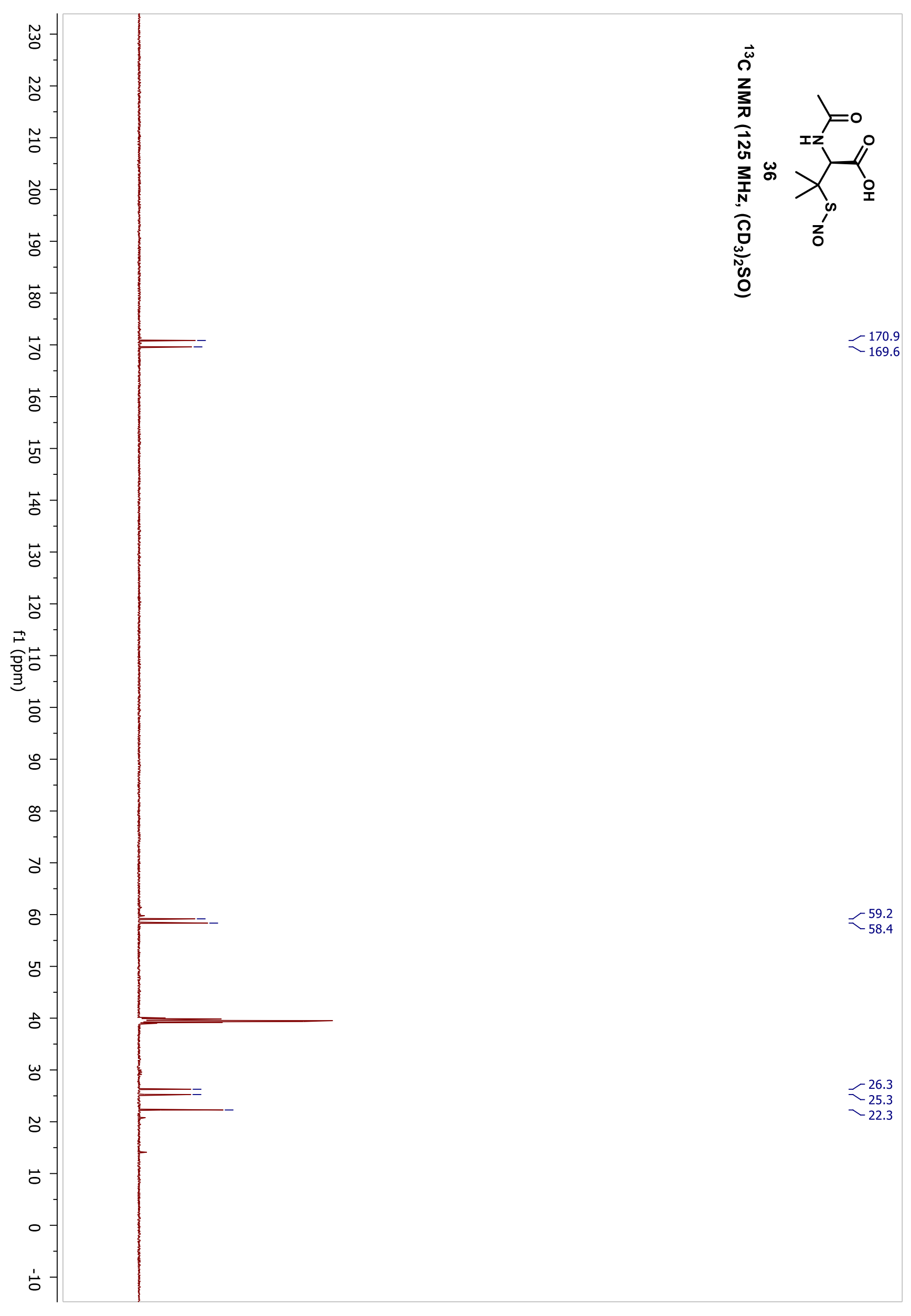

参 


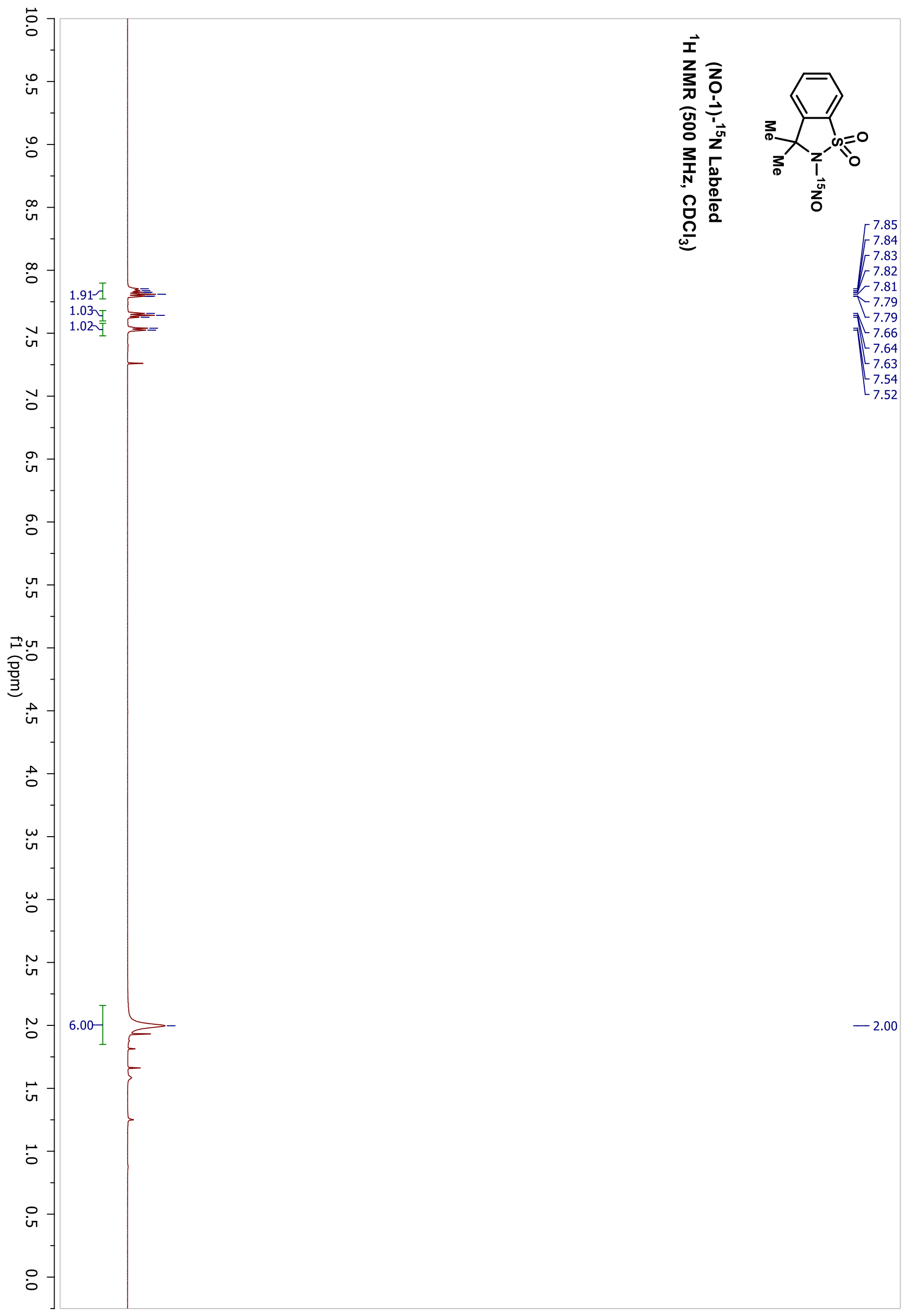




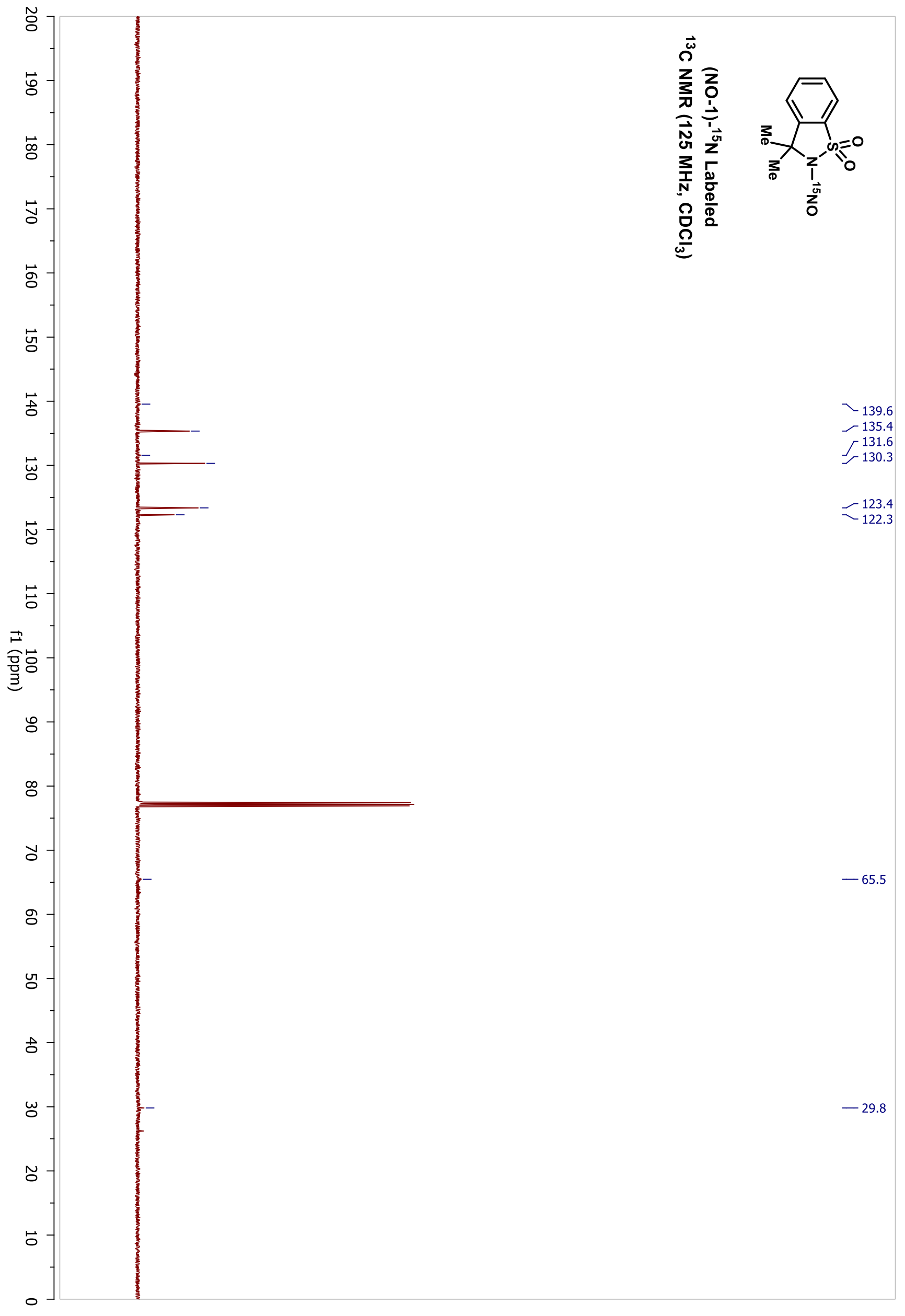

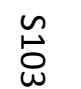




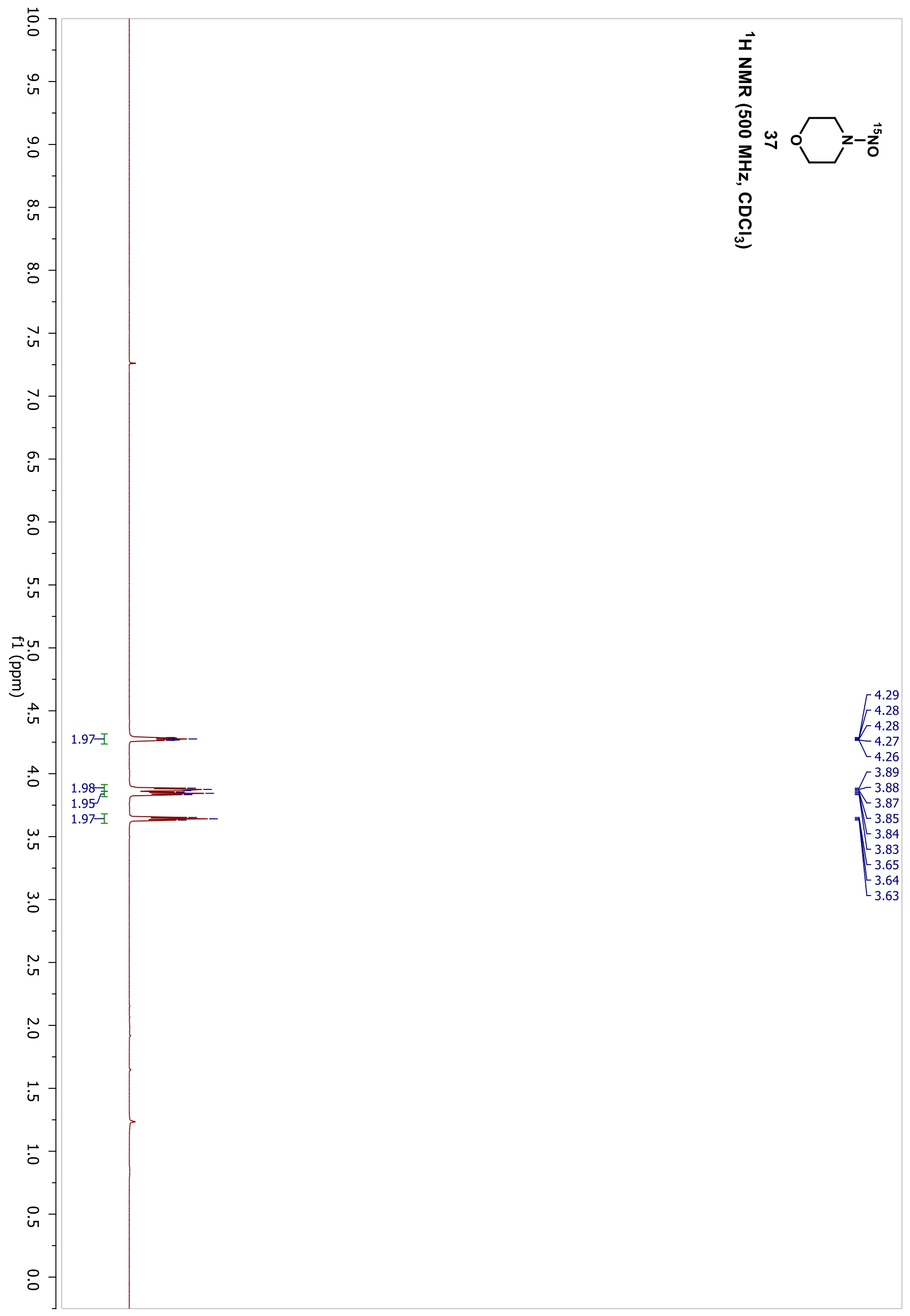




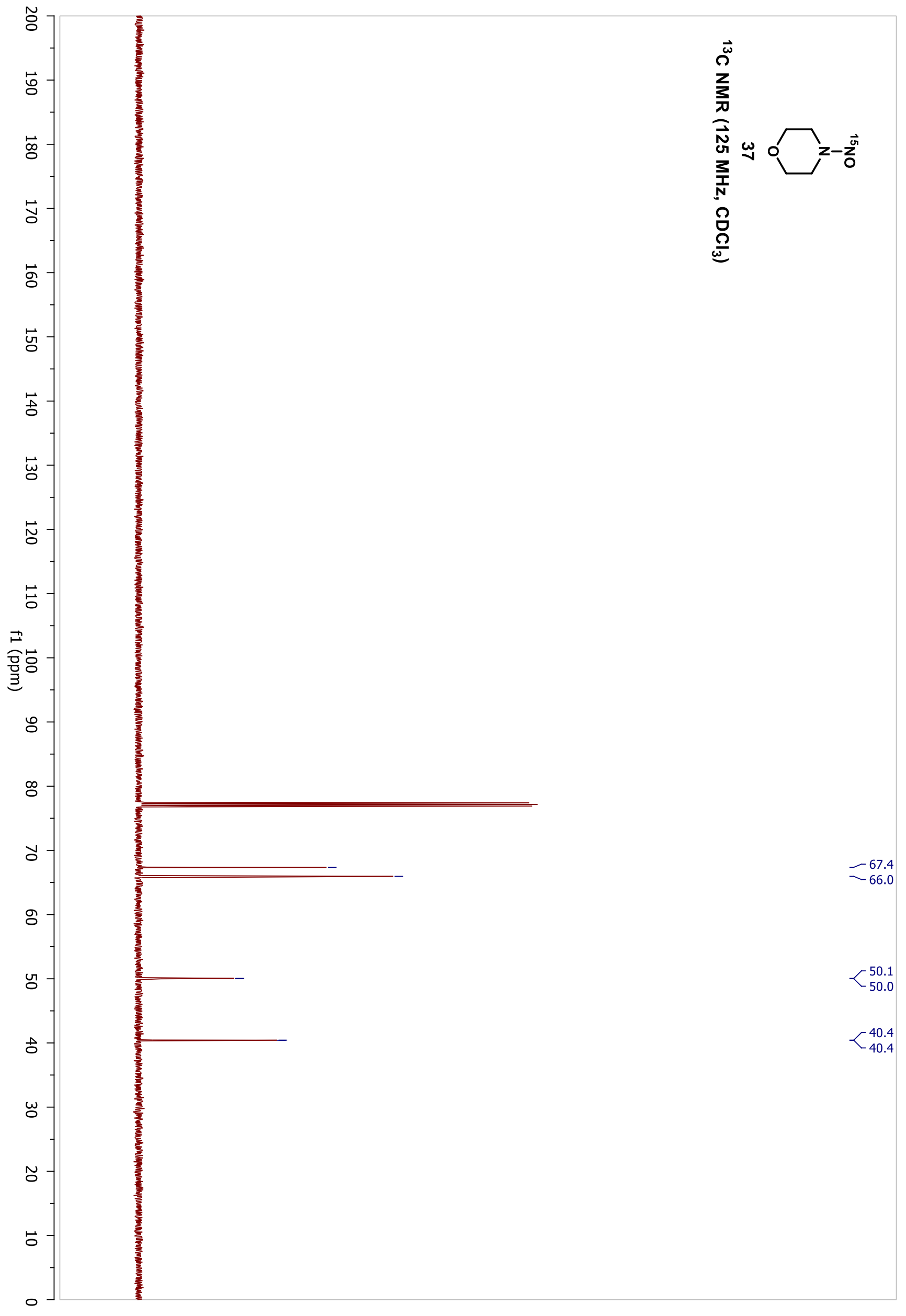

参 


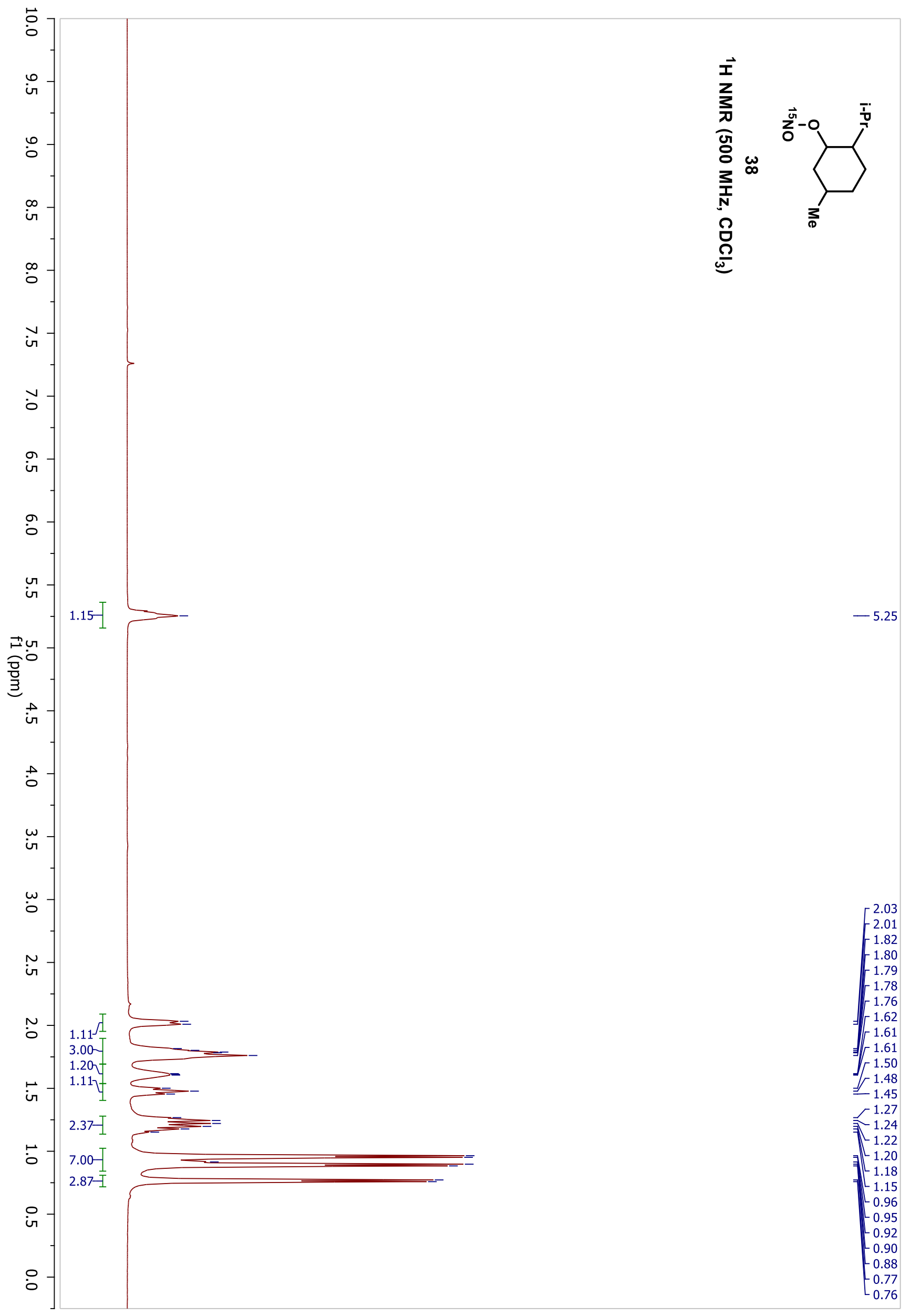




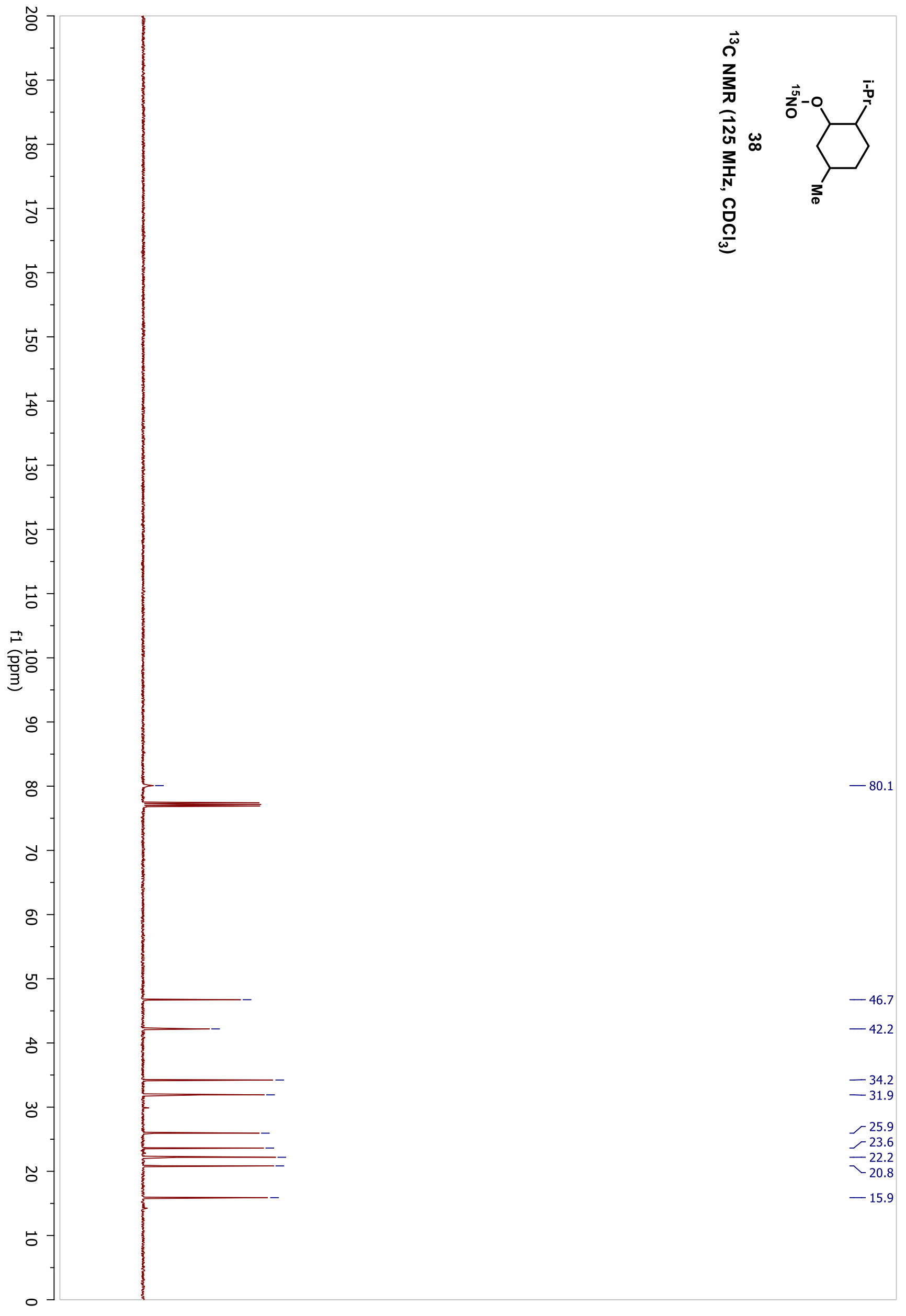

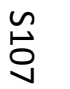




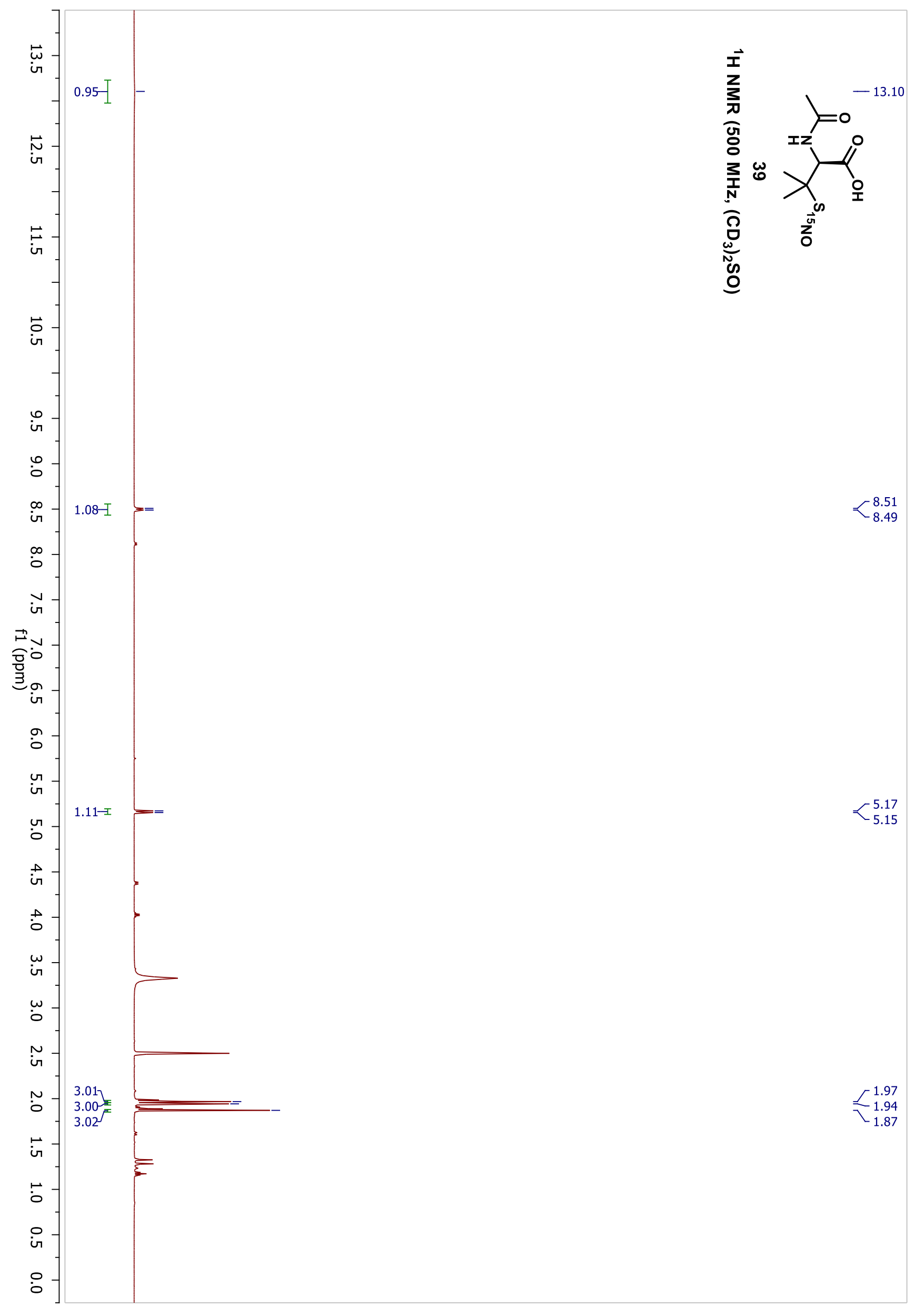

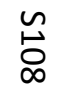




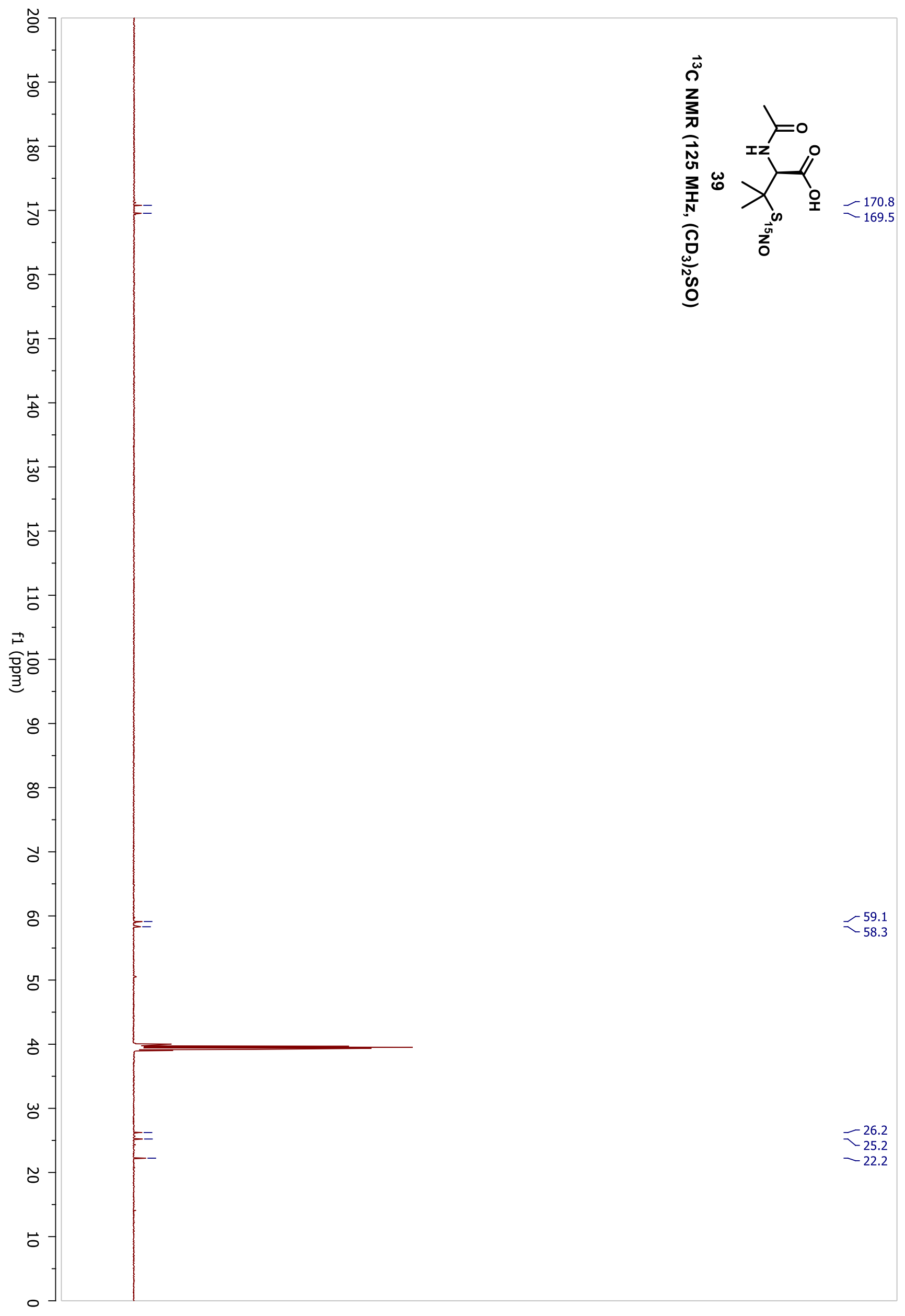




\section{Calculation Set Up}

Geometries for the NO-1a, NO-1a $\left(\mathrm{sp}^{3}\right)$, and NO-1b reagent were all calculated using the $\mathrm{B} 3 \mathrm{LYP} / 6-311+\mathrm{G}(2 \mathrm{p}, \mathrm{d})$ model chemistry with the PCM(acetonitrile) solvent model.[1,3,4] Additionally, vibrational frequency analysis was performed to confirm that all geometries were true minima.[2] Once the ground state geometries were confirmed the Nitrogen-Nitrogen bond dihedral angle was scanned/rotated using a scan job in gaussian, changing the dihedral angle by ten degrees all the way until three-hundred sixty degrees is reached, calculating a ground state energy. The NO-1a was scanned constrained to $\mathrm{sp}^{2}$ hybridization and constrained to $\mathrm{sp}^{3}$ hybridization represented as the green and blue curve respectively in the bottom of Figure 4 . While NO-1a unconstrained to any hybridization was scanned to produce the top of Figure 4. Scheme SC1 shows the scanned coordinate and potential energy surface path.

\section{Optimized Geometries}

Scheme S1. Coordinate Scan<smiles>CC1(C)c2ccccc2S(=O)(=O)[N+]1=NO</smiles>

NO-1a<smiles>CC1(C)c2ccccc2S(=O)(=O)N1N=O</smiles>

NO-1a $\left(\mathrm{sp}^{3}\right)$<smiles>CC1(C)c2ccccc2S(=O)(=O)N1/N=N/[O-]</smiles>

NO-1b

Table S1. Optimized Geometries of NO-1a $\left(s p^{2}\right), N O-1\left(s p^{3}\right)$ and NO-1b $\left(s p^{2}\right)$

NO-1a

\begin{tabular}{|llll|}
\hline $\mathrm{C}$ & -0.933083 & -0.727194 & 0.000033 \\
$\mathrm{C}$ & -1.058869 & 0.653554 & 0.000094 \\
$\mathrm{C}$ & -2.341557 & 1.195960 & 0.000182 \\
$\mathrm{C}$ & -3.444025 & 0.349781 & 0.000209 \\
$\mathrm{C}$ & -3.288206 & -1.037978 & 0.000155 \\
$\mathrm{C}$ & -2.018543 & -1.595824 & 0.000066 \\
$\mathrm{C}$ & 0.224207 & 1.469915 & 0.000070 \\
$\mathrm{H}$ & -2.485907 & 2.268836 & 0.000227 \\
$\mathrm{H}$ & -4.439564 & 0.775365 & 0.000274 \\
$\mathrm{H}$ & -4.158114 & -1.681716 & 0.000184 \\
$\mathrm{H}$ & -1.879742 & -2.669187 & 0.000027 \\
$\mathrm{~N}$ & 1.291554 & 0.420849 & -0.000050 \\
\hline
\end{tabular}

\begin{tabular}{|llll|}
\hline $\mathrm{S}$ & 0.746074 & -1.263299 & -0.000093 \\
$\mathrm{O}$ & 1.114532 & -1.900630 & -1.246495 \\
$\mathrm{O}$ & 1.114659 & -1.900792 & 1.246188 \\
$\mathrm{~N}$ & 2.563560 & 0.761552 & -0.000123 \\
$\mathrm{O}$ & 3.366339 & -0.163605 & -0.000315 \\
$\mathrm{C}$ & 0.348161 & 2.323369 & -1.270527 \\
$\mathrm{H}$ & -0.454626 & 3.060496 & -1.297233 \\
$\mathrm{H}$ & 1.300982 & 2.852965 & -1.273130 \\
$\mathrm{H}$ & 0.282299 & 1.703862 & -2.165369 \\
$\mathrm{C}$ & 0.348298 & 2.323220 & 1.270754 \\
$\mathrm{H}$ & 1.301114 & 2.852826 & 1.273311 \\
$\mathrm{H}$ & -0.454493 & 3.060335 & 1.297638 \\
$\mathrm{H}$ & 0.282545 & 1.703606 & 2.165530 \\
\hline
\end{tabular}


NO-1a $\left(\mathrm{sp}^{3}\right)$

NO-1b

\begin{tabular}{|llll|}
\hline C & 1.098032 & -0.584041 & -0.000222 \\
$\mathrm{C}$ & 0.870591 & 0.783703 & -0.000023 \\
$\mathrm{C}$ & 1.975677 & 1.631523 & -0.000086 \\
$\mathrm{C}$ & 3.255541 & 1.090132 & -0.000350 \\
$\mathrm{C}$ & 3.454395 & -0.292235 & -0.000552 \\
$\mathrm{C}$ & 2.366598 & -1.152251 & -0.000488 \\
$\mathrm{H}$ & 1.845128 & 2.706135 & 0.000073 \\
$\mathrm{H}$ & 4.111992 & 1.752509 & -0.000395 \\
$\mathrm{H}$ & 4.458364 & -0.696048 & -0.000760 \\
$\mathrm{H}$ & 2.502295 & -2.225848 & -0.000637 \\
$\mathrm{~S}$ & -0.396144 & -1.509953 & -0.000084 \\
$\mathrm{C}$ & -0.896596 & 2.048510 & 1.275927 \\
$\mathrm{H}$ & -0.269379 & 2.940245 & 1.303635 \\
$\mathrm{H}$ & -1.938946 & 2.359939 & 1.284301 \\
$\mathrm{H}$ & -0.691134 & 1.454232 & 2.166546 \\
$\mathrm{C}$ & -0.896953 & 2.048723 & -1.275248 \\
$\mathrm{H}$ & -1.939397 & 2.359848 & -1.283439 \\
$\mathrm{H}$ & -0.270011 & 2.940657 & -1.302796 \\
$\mathrm{H}$ & -0.691401 & 1.454725 & -2.166034 \\
$\mathrm{O}$ & -0.617402 & -2.222191 & 1.240512 \\
$\mathrm{O}$ & -0.617796 & -2.221973 & -1.240734 \\
$\mathrm{~N}$ & -2.648274 & -0.261763 & 0.000339 \\
$\mathrm{~N}$ & -1.337862 & -0.042993 & 0.000204 \\
$\mathrm{C}$ & -0.578381 & 1.252433 & 0.000228 \\
$\mathrm{O}$ & -3.361010 & 0.730061 & 0.000464 \\
\hline
\end{tabular}

\begin{tabular}{|llll|}
\hline C & -0.932566 & -0.728299 & -0.017228 \\
C & -1.061717 & 0.649635 & -0.036856 \\
C & -2.345722 & 1.187769 & -0.029639 \\
C & -3.444073 & 0.334963 & -0.004711 \\
C & -3.282566 & -1.051729 & 0.010066 \\
C & -2.009868 & -1.604747 & 0.003267 \\
C & 0.228589 & 1.452488 & -0.021230 \\
H & -2.496339 & 2.259138 & -0.046809 \\
H & -4.441649 & 0.755855 & -0.003000 \\
H & -4.149929 & -1.698827 & 0.021439 \\
H & -1.862866 & -2.676802 & 0.007739 \\
N & 1.275399 & 0.464334 & -0.497864 \\
S & 0.754051 & -1.228800 & -0.002601 \\
O & 1.047633 & -2.179219 & -1.052525 \\
O & 1.210930 & -1.496794 & 1.346034 \\
N & 2.547824 & 0.733903 & -0.141135 \\
O & 3.294036 & -0.223268 & -0.115508 \\
C & 0.206877 & 2.610218 & -1.026372 \\
H & -0.540009 & 3.349016 & -0.734922 \\
H & 1.179003 & 3.104041 & -1.042754 \\
H & -0.024545 & 2.251415 & -2.029454 \\
C & 0.526530 & 1.958233 & 1.401119 \\
H & 1.452083 & 2.533309 & 1.413215 \\
H & -0.287931 & 2.606217 & 1.726287 \\
H & 0.611119 & 1.132854 & 2.108360 \\
\hline & & &
\end{tabular}

Table S2. Total energy values for the singlet in Scheme 1 calculated with B3LYP/6-311+G(2p,d) model chemistry.

\begin{tabular}{|c|c|c|c|}
\hline Structure & $\begin{array}{c}\text { SCF Energy } \\
\text { (Hartree) }\end{array}$ & $\begin{array}{c}\text { Thermal Correction } \\
\text { (Hartree) }\end{array}$ & $\begin{array}{c}\text { ZPE Correction } \\
\text { (Hartree) }\end{array}$ \\
\hline $\begin{array}{c}\text { NO-1a } \\
\text { with } \\
\text { PCM (MeCN) }\end{array}$ & -1082.466148 & -1082.266828 & -1082.280440 \\
\hline $\begin{array}{c}\text { NO-1b } \\
\text { with } \\
\text { PCM (MeCN) }\end{array}$ & -1082.464226 & -1082.264849 & -1082.278433 \\
\hline $\begin{array}{c}\text { NO-1a (sp }{ }^{3} \text { ) } \\
\text { with } \\
\text { PCM (MeCN) }\end{array}$ & -1082.456008 & -1082.257089 & -1082.270638 \\
\hline
\end{tabular}




\section{Full Gaussian Citation}

As stated previously in this SI document and in the communication text, a local development version of the GAUSSIAN suite of electronic structure programs was used for all calculations. The full citation for the program is:

M. J. Frisch, G. W. Trucks, H. B. Schlegel, G. E. Scuseria, M. A. Robb, J. R. Cheeseman, G. Scalmani, V. Barone, G. A. Petersson, H. Nakatsuji, X. Li, M. Caricato, A. V. Marenich, J. Bloino, B. G. Janesko, R. Gomperts, B. Mennucci, H. P. Hratchian, J. V. Ortiz, A. F. Izmaylov, J. L. Sonnenberg, D. Williams-Young, F. Ding, F. Lipparini, F. Egidi, J. Goings, B. Peng, A. Petrone, T. Henderson, D. Ranasinghe, V. G. Zakrzewski, J. Gao, N. Rega, G. Zheng, W. Liang, M. Hada, M. Ehara, K. Toyota, R. Fukuda, J. Hasegawa, M. Ishida, T. Nakajima, Y. Honda, O. Kitao, H. Nakai, T. Vreven, K. Throssell, J. A. Montgomery, Jr., J. E. Peralta, F. Ogliaro, M. J. Bearpark, J. J. Heyd, E. N. Brothers, K. N. Kudin, V. N. Staroverov, T. A. Keith, R. Kobayashi, J. Normand, K. Raghavachari, A. P. Rendell, J. C. Burant, S. S. Iyengar, J. Tomasi, M. Cossi, J. M. Millam, M. Klene, C. Adamo, R. Cammi, J. W. Ochterski, R. L. Martin, K. Morokuma, O. Farkas, J. B. Foresman, and D. J. Fox, GAUSSIAN, Development Version, Revision I.10+, Gaussian, Inc., Wallingford CT, 2016.

\section{References}

1. Hratchian, H. P.; Schlegel, H. B. Finding Minima, Transition States, and Following Reaction Pathways on Ab Initio Potential Energy Surfaces. In Theory and Applications of Computational Chemistry; Elsevier, 2005; pp 195-249.

2. Marenich, A. V.; Cramer, C. J.; Truhlar, D. G. Universal Solvation Model Based on Solute Electron Density and on a Continuum Model of the Solvent Defined by the Bulk Dielectric Constant and Atomic Surface Tensions. J. Phys. Chem. B 2009, 113 (18), 6378-6396.

3. Becke, A. D. Density functional Thermochemistry. III. The Role of Exact Exchange. $J$. Chem. Phys. 1993, 98 (7), 5648-5652.

4. Wang, Y.; Perdew, J. P. Spin Scaling of the Electron-Gas Correlation Energy in the HighDensity Limit. Phys. Rev. B Condens. Matter 1991, 43 (11), 8911-8916. 


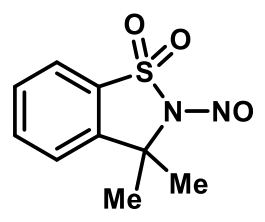

NO-1

\section{3,3-dimethyl-2-nitroso-2,3-dihydrobenzo[d]isothiazole 1,1-dioxide (NO-1)}

NO-1 was dissolved completely in dichloromethane in a $20 \mathrm{ml}$ scintillation vial. This sample was allowed to slowly evaporate over the course of 24 hours to produce yellow crystals of suitable quality for x-ray analysis. Measurements were taken as described below.

A yellow block with approximate orthogonal dimensions $0.060 \times 0.154 \times 0.338 \mathrm{~mm}^{3}$ was placed and optically centered on the Bruker Duo ${ }^{1}$ APEXII CCD system at $-183^{\circ} \mathrm{C}(90 \mathrm{~K})$. Indexing of the unit cell used a random set of reflections collected from three series of $0.5^{\circ}$ wide $\omega$-scans, 10 seconds per frame, and 30 frames per series that were well distributed in reciprocal space. Five $\omega$-scan data frame series were collected $\left[\mathrm{MoK}_{a}\right]$ with $0.3^{\circ}$ wide scans, 15 seconds per frame and 606 frames collected per series at varying $\varphi$ angles $\left(\varphi=0^{\circ}, 72^{\circ}, 144^{\circ}, 216^{\circ}, 288^{\circ}\right)$. The crystal to detector distance was $5.15 \mathrm{~cm}$, thus providing a complete sphere of data to $2 \theta_{\max }=61.01^{\circ}$.

\section{Structural determination and Refinement:}

All crystallographic calculations were performed on an Intel Xeon E5-1620v2 at 3.70GHz an eight core processor and 16GB of extended memory. Data collected were corrected for Lorentz and polarization effects with Saint ${ }^{1}$ and absorption using Blessing's method and merged as incorporated with the program Sadabs ${ }^{2,3}$. The SHELXTL ${ }^{4}$ program package was implemented to determine the probable space group and set up the initial files. System symmetry, systematic absences and intensity statistics indicated the centrosymmetric monoclinic space group $\mathrm{P} 2{ }_{1} / \mathrm{n}$ (no. 14). The structure was determined by direct methods with the non-hydrogen atoms being located directly for the molecule using the program $\mathrm{XT}^{5}$. The structure was refined with $\mathrm{XL}^{6}$. The 20407 data collected were merged, based upon identical indices to 11861 data, then for least squares refinement to 3091 unique data $[\mathrm{R}(\mathrm{int})=0.0224]$. All non-hydrogen atoms were refined anisotropically. A disorder was modeled for the terminal $\mathrm{N}-\mathrm{O}$, group with the final ratio $0.87: 0.13$. Hydrogen atoms were idealized initially and then allowed to refine freely throughout the final refinement stage. The final structure was refined to convergence with $\mathrm{R}(\mathrm{F})=3.63 \%$, $\mathrm{wR}\left(\mathrm{F}^{2}\right)=8.27 \%$, GOF $=1.065$ for all 3091 unique reflections $\left[\mathrm{R}(\mathrm{F})=3.08, \mathrm{wR}\left(\mathrm{F}^{2}\right)=7.87 \%\right.$ for those 2706 data with $\mathrm{Fo}>4 \sigma(\mathrm{Fo})]$. The final difference-Fourier map was featureless indicating that the structure is both correct and complete.

Table S3. Crystal data and structure refinement for

$\left[\mathrm{C}_{9} \mathrm{H}_{10} \mathrm{~N}_{2} \mathrm{O}_{3} \mathrm{~S}\right]$. Identification code JF2913FMI (JDGR2)

Empirical formula

C9 H10 N2 O3 S

Formula weight

226.25 
Temperature

Wavelength

Crystal system

Space group

Unit cell dimensions

Volume

Z

Density (calculated)

Absorption coefficient

$\mathrm{F}(000)$

Crystal size

Crystal color and habit

Diffractometer

Theta range for data collection

Index ranges

Reflections collected

Independent reflections

Observed reflections (I $>2$ sigma(I))

Completeness to theta $=25.242^{\circ}$

Absorption correction

Max. and min. transmission

Solution method

Refinement method

Data / restraints / parameters

Goodness-of-fit on $\mathrm{F}^{2}$

Final $\mathrm{R}$ indices [I>2sigma(I)]

$\mathrm{R}$ indices (all data)

Largest diff. peak and hole
90(2) K

$0.71073 \AA$

Monoclinic

$\mathrm{P} 2{ }_{1} / \mathrm{n}$

$\mathrm{a}=7.7236(7) \AA \quad \alpha=90^{\circ}$.

$\mathrm{b}=9.8965(9) \AA \quad \beta=94.6681(13)^{\circ}$.

$\mathrm{c}=13.3301(12) \AA \quad \gamma=90^{\circ}$.

1015.53(16) $\AA^{3}$

4

$1.480 \mathrm{Mg} / \mathrm{m}^{3}$

$0.307 \mathrm{~mm}^{-1}$

472

$0.338 \times 0.154 \times 0.060 \mathrm{~mm}^{3}$

Yellow Block

Bruker APEX-II CCD

2.566 to $30.509^{\circ}$.

$-11<=\mathrm{h}<=11,-14<=\mathrm{k}<=14,-19<=\mathrm{l}<=19$

11861

$3091[\mathrm{R}(\mathrm{int})=0.0224]$

2706

$100.0 \%$

Semi-empirical from equivalents

0.9575 and 0.8766

SHELXT (Sheldrick, 2014)

SHELXL-2018/3 (Sheldrick, 2018) Full-matrix least-squares on F ${ }^{2}$

$3091 / 14 / 194$

1.065

$\mathrm{R} 1=0.0308, \mathrm{wR} 2=0.0787$

$\mathrm{R} 1=0.0363, \mathrm{wR} 2=0.0827$

0.470 and -0.392 e. $\AA^{-3}$ 
Table S4. Atomic coordinates ( x 104) and equivalent isotropic displacement parameters $\left(\AA^{2} \mathrm{x}\right.$ $10^{3}$ ) for JF2913FMI. U(eq) is defined as one third of the trace of the orthogonalized $\mathrm{U}^{\mathrm{ij}}$ tensor.

\begin{tabular}{lcccc}
\hline & $\mathrm{x}$ & $\mathrm{y}$ & $\mathrm{z}$ & $\mathrm{U}(\mathrm{eq})$ \\
\hline $\mathrm{S}(1)$ & $4763(1)$ & $2078(1)$ & $2767(1)$ & $15(1)$ \\
$\mathrm{O}(1)$ & $3101(1)$ & $1688(1)$ & $3080(1)$ & $27(1)$ \\
$\mathrm{O}(2)$ & $4988(1)$ & $2114(1)$ & $1712(1)$ & $26(1)$ \\
$\mathrm{N}(1)$ & $6354(1)$ & $1119(1)$ & $3387(1)$ & $14(1)$ \\
$\mathrm{N}(2)$ & $6463(5)$ & $-229(2)$ & $3272(2)$ & $20(1)$ \\
$\mathrm{O}(3)$ & $5421(1)$ & $-686(1)$ & $2611(1)$ & $25(1)$ \\
$\mathrm{N}(2 \mathrm{~B})$ & $6240(30)$ & $-193(10)$ & $3105(16)$ & $19(2)$ \\
$\mathrm{O}(3 \mathrm{~B})$ & $7244(9)$ & $-925(7)$ & $3622(6)$ & $26(1)$ \\
$\mathrm{C}(1)$ & $7391(1)$ & $1780(1)$ & $4250(1)$ & $12(1)$ \\
$\mathrm{C}(2)$ & $6875(1)$ & $3255(1)$ & $4121(1)$ & $13(1)$ \\
$\mathrm{C}(3)$ & $5548(1)$ & $3530(1)$ & $3384(1)$ & $14(1)$ \\
$\mathrm{C}(4)$ & $6827(2)$ & $1211(1)$ & $5241(1)$ & $16(1)$ \\
$\mathrm{C}(5)$ & $9330(1)$ & $1573(1)$ & $4149(1)$ & $18(1)$ \\
$\mathrm{C}(6)$ & $4826(1)$ & $3157(1)$ & $20(1)$ \\
$\mathrm{C}(7)$ & $5931(2)$ & $5886(1)$ & $3705(1)$ & $24(1)$ \\
$\mathrm{C}(8)$ & $5712(2)$ & $5636(1)$ & $4460(1)$ & $23(1)$ \\
$\mathrm{C}(9)$ & $7329(1)$ & $4680(1)$ & $18(1)$ \\
& & & & \\
\hline
\end{tabular}


Table S5. Bond lengths $[\AA]$ and angles $\left[{ }^{\circ}\right]$ for JF2913FMI.

\begin{tabular}{|c|c|c|c|}
\hline $\mathrm{S}(1)-\mathrm{O}(2)$ & $1.4308(9)$ & $\mathrm{N}(1)-\mathrm{S}(1)-\mathrm{C}(3)$ & $91.20(5)$ \\
\hline $\mathrm{S}(1)-\mathrm{O}(1)$ & $1.4346(9)$ & $\mathrm{N}(2)-\mathrm{N}(1)-\mathrm{C}(1)$ & $119.06(11)$ \\
\hline $\mathrm{S}(1)-\mathrm{N}(1)$ & $1.7109(9)$ & $\mathrm{N}(2 \mathrm{~B})-\mathrm{N}(1)-\mathrm{C}(1)$ & $130.6(6)$ \\
\hline $\mathrm{S}(1)-\mathrm{C}(3)$ & $1.7400(11)$ & $\mathrm{N}(2)-\mathrm{N}(1)-\mathrm{S}(1)$ & $122.93(11)$ \\
\hline $\mathrm{N}(1)-\mathrm{N}(2)$ & $1.3461(18)$ & $\mathrm{N}(2 \mathrm{~B})-\mathrm{N}(1)-\mathrm{S}(1)$ & $111.8(6)$ \\
\hline $\mathrm{N}(1)-\mathrm{N}(2 \mathrm{~B})$ & $1.352(9)$ & $\mathrm{C}(1)-\mathrm{N}(1)-\mathrm{S}(1)$ & $116.55(7)$ \\
\hline $\mathrm{N}(1)-\mathrm{C}(1)$ & $1.4976(13)$ & $\mathrm{O}(3)-\mathrm{N}(2)-\mathrm{N}(1)$ & $113.74(15)$ \\
\hline $\mathrm{N}(2)-\mathrm{O}(3)$ & $1.230(2)$ & $\mathrm{O}(3 \mathrm{~B})-\mathrm{N}(2 \mathrm{~B})-\mathrm{N}(1)$ & $112.8(11)$ \\
\hline $\mathrm{N}(2 \mathrm{~B})-\mathrm{O}(3 \mathrm{~B})$ & $1.229(10)$ & $\mathrm{N}(1)-\mathrm{C}(1)-\mathrm{C}(2)$ & $102.34(8)$ \\
\hline $\mathrm{C}(1)-\mathrm{C}(2)$ & $1.5196(14)$ & $\mathrm{N}(1)-\mathrm{C}(1)-\mathrm{C}(5)$ & $109.90(9)$ \\
\hline $\mathrm{C}(1)-\mathrm{C}(5)$ & $1.5283(15)$ & $\mathrm{C}(2)-\mathrm{C}(1)-\mathrm{C}(5)$ & 111.63(9) \\
\hline $\mathrm{C}(1)-\mathrm{C}(4)$ & $1.5324(15)$ & $\mathrm{N}(1)-\mathrm{C}(1)-\mathrm{C}(4)$ & $109.30(8)$ \\
\hline $\mathrm{C}(2)-\mathrm{C}(3)$ & $1.3877(15)$ & $\mathrm{C}(2)-\mathrm{C}(1)-\mathrm{C}(4)$ & $111.10(9)$ \\
\hline $\mathrm{C}(2)-\mathrm{C}(9)$ & $1.3955(15)$ & $\mathrm{C}(5)-\mathrm{C}(1)-\mathrm{C}(4)$ & $112.11(9)$ \\
\hline $\mathrm{C}(3)-\mathrm{C}(6)$ & $1.3931(15)$ & $\mathrm{C}(3)-\mathrm{C}(2)-\mathrm{C}(9)$ & $118.54(10)$ \\
\hline $\mathrm{C}(4)-\mathrm{H}(4 \mathrm{~A})$ & $0.976(15)$ & $\mathrm{C}(3)-\mathrm{C}(2)-\mathrm{C}(1)$ & 116.11(9) \\
\hline $\mathrm{C}(4)-\mathrm{H}(4 \mathrm{~B})$ & $0.969(16)$ & $\mathrm{C}(9)-\mathrm{C}(2)-\mathrm{C}(1)$ & $125.35(10)$ \\
\hline $\mathrm{C}(4)-\mathrm{H}(4 \mathrm{C})$ & $0.964(17)$ & $C(2)-C(3)-C(6)$ & $123.65(10)$ \\
\hline $\mathrm{C}(5)-\mathrm{H}(5 \mathrm{~A})$ & $0.986(17)$ & $\mathrm{C}(2)-\mathrm{C}(3)-\mathrm{S}(1)$ & $112.43(8)$ \\
\hline $\mathrm{C}(5)-\mathrm{H}(5 \mathrm{~B})$ & $0.938(18)$ & $\mathrm{C}(6)-\mathrm{C}(3)-\mathrm{S}(1)$ & 123.91(9) \\
\hline $\mathrm{C}(5)-\mathrm{H}(5 \mathrm{C})$ & $0.968(17)$ & $\mathrm{C}(1)-\mathrm{C}(4)-\mathrm{H}(4 \mathrm{~A})$ & $110.8(9)$ \\
\hline$C(6)-C(7)$ & $1.3877(18)$ & $\mathrm{C}(1)-\mathrm{C}(4)-\mathrm{H}(4 \mathrm{~B})$ & 109.1(9) \\
\hline $\mathrm{C}(6)-\mathrm{H}(6)$ & $0.956(16)$ & $\mathrm{H}(4 \mathrm{~A})-\mathrm{C}(4)-\mathrm{H}(4 \mathrm{~B})$ & $109.6(13)$ \\
\hline $\mathrm{C}(7)-\mathrm{C}(8)$ & $1.394(2)$ & $\mathrm{C}(1)-\mathrm{C}(4)-\mathrm{H}(4 \mathrm{C})$ & 109.7(9) \\
\hline $\mathrm{C}(7)-\mathrm{H}(7)$ & $0.924(19)$ & $\mathrm{H}(4 \mathrm{~A})-\mathrm{C}(4)-\mathrm{H}(4 \mathrm{C})$ & $108.2(13)$ \\
\hline $\mathrm{C}(8)-\mathrm{C}(9)$ & $1.3939(17)$ & $\mathrm{H}(4 \mathrm{~B})-\mathrm{C}(4)-\mathrm{H}(4 \mathrm{C})$ & $109.4(13)$ \\
\hline $\mathrm{C}(8)-\mathrm{H}(8)$ & $0.926(18)$ & $\mathrm{C}(1)-\mathrm{C}(5)-\mathrm{H}(5 \mathrm{~A})$ & $108.7(10)$ \\
\hline \multirow[t]{2}{*}{$\mathrm{C}(9)-\mathrm{H}(9)$} & $0.959(15)$ & $\mathrm{C}(1)-\mathrm{C}(5)-\mathrm{H}(5 \mathrm{~B})$ & 109.6(11) \\
\hline & & $\mathrm{H}(5 \mathrm{~A})-\mathrm{C}(5)-\mathrm{H}(5 \mathrm{~B})$ & $109.8(14)$ \\
\hline $\mathrm{O}(2)-\mathrm{S}(1)-\mathrm{O}(1)$ & $118.40(6)$ & $\mathrm{C}(1)-\mathrm{C}(5)-\mathrm{H}(5 \mathrm{C})$ & $112.1(10)$ \\
\hline $\mathrm{O}(2)-\mathrm{S}(1)-\mathrm{N}(1)$ & $110.40(5)$ & $\mathrm{H}(5 \mathrm{~A})-\mathrm{C}(5)-\mathrm{H}(5 \mathrm{C})$ & $108.9(14)$ \\
\hline $\mathrm{O}(1)-\mathrm{S}(1)-\mathrm{N}(1)$ & $109.57(5)$ & $\mathrm{H}(5 \mathrm{~B})-\mathrm{C}(5)-\mathrm{H}(5 \mathrm{C})$ & $107.7(14)$ \\
\hline $\mathrm{O}(2)-\mathrm{S}(1)-\mathrm{C}(3)$ & $112.20(6)$ & $C(7)-C(6)-C(3)$ & $117.06(11)$ \\
\hline $\mathrm{O}(1)-\mathrm{S}(1)-\mathrm{C}(3)$ & $111.73(5)$ & $\mathrm{C}(7)-\mathrm{C}(6)-\mathrm{H}(6)$ & $122.9(10)$ \\
\hline
\end{tabular}




$\begin{array}{llll}\mathrm{C}(3)-\mathrm{C}(6)-\mathrm{H}(6) & 120.0(10) & \mathrm{C}(9)-\mathrm{C}(8)-\mathrm{H}(8) & 119.6(11) \\ \mathrm{C}(6)-\mathrm{C}(7)-\mathrm{C}(8) & 120.44(11) & \mathrm{C}(7)-\mathrm{C}(8)-\mathrm{H}(8) & 118.8(11) \\ \mathrm{C}(6)-\mathrm{C}(7)-\mathrm{H}(7) & 119.5(11) & \mathrm{C}(8)-\mathrm{C}(9)-\mathrm{C}(2) & 118.70(11) \\ \mathrm{C}(8)-\mathrm{C}(7)-\mathrm{H}(7) & 120.1(11) & \mathrm{C}(8)-\mathrm{C}(9)-\mathrm{H}(9) & 121.4(9) \\ \mathrm{C}(9)-\mathrm{C}(8)-\mathrm{C}(7) & 121.59(11) & \mathrm{C}(2)-\mathrm{C}(9)-\mathrm{H}(9) & 119.9(9)\end{array}$

Symmetry transformations used to generate equivalent atoms: 
Table S6. Anisotropic displacement parameters $\left(\AA^{2} \times 10^{3}\right)$ for JF2913FMI. The anisotropic displacement factor exponent takes the form: $-2 \pi^{2}\left[h^{2} a^{* 2} U^{11}+\ldots+2 h k a * b * U^{12}\right]$

\begin{tabular}{lcccccc}
\hline & $\mathrm{U}^{11}$ & $\mathrm{U}^{22}$ & $\mathrm{U}^{33}$ & $\mathrm{U}^{23}$ & $\mathrm{U}^{13}$ & $\mathrm{U}^{12}$ \\
\hline $\mathrm{S}(1)$ & $13(1)$ & $19(1)$ & $14(1)$ & $-2(1)$ & $-2(1)$ & $2(1)$ \\
$\mathrm{O}(1)$ & $14(1)$ & $29(1)$ & $38(1)$ & $-8(1)$ & $1(1)$ & $-4(1)$ \\
$\mathrm{O}(2)$ & $33(1)$ & $32(1)$ & $12(1)$ & $-2(1)$ & $-4(1)$ & $9(1)$ \\
$\mathrm{N}(1)$ & $16(1)$ & $12(1)$ & $14(1)$ & $-1(1)$ & $-1(1)$ & $1(1)$ \\
$\mathrm{N}(2)$ & $27(1)$ & $14(1)$ & $19(1)$ & $-2(1)$ & $5(1)$ & $-1(1)$ \\
$\mathrm{O}(3)$ & $32(1)$ & $21(1)$ & $23(1)$ & $-9(1)$ & $4(1)$ & $-8(1)$ \\
$\mathrm{N}(2 \mathrm{~B})$ & $25(3)$ & $14(3)$ & $20(3)$ & $2(2)$ & $9(3)$ & $2(2)$ \\
$\mathrm{O}(3 \mathrm{~B})$ & $27(3)$ & $20(3)$ & $31(3)$ & $-1(3)$ & $4(3)$ & $3(3)$ \\
$\mathrm{C}(1)$ & $12(1)$ & $13(1)$ & $12(1)$ & $0(1)$ & $-1(1)$ & $0(1)$ \\
$\mathrm{C}(2)$ & $14(1)$ & $13(1)$ & $13(1)$ & $0(1)$ & $4(1)$ & $-1(1)$ \\
$\mathrm{C}(3)$ & $14(1)$ & $14(1)$ & $14(1)$ & $0(1)$ & $3(1)$ & $1(1)$ \\
$\mathrm{C}(4)$ & $19(1)$ & $17(1)$ & $14(1)$ & $2(1)$ & $1(1)$ & $-3(1)$ \\
$\mathrm{C}(5)$ & $13(1)$ & $21(1)$ & $21(1)$ & $2(1)$ & $1(1)$ & $2(1)$ \\
$\mathrm{C}(6)$ & $23(1)$ & $19(1)$ & $18(1)$ & $5(1)$ & $6(1)$ & $7(1)$ \\
$\mathrm{C}(7)$ & $33(1)$ & $13(1)$ & $27(1)$ & $3(1)$ & $15(1)$ & $4(1)$ \\
$\mathrm{C}(8)$ & $29(1)$ & $14(1)$ & $26(1)$ & $-4(1)$ & $12(1)$ & $-7(1)$ \\
$\mathrm{C}(9)$ & $19(1)$ & $17(1)$ & $18(1)$ & $-2(1)$ & $4(1)$ & $-5(1)$ \\
& & & & & & \\
\hline
\end{tabular}


Table S7. Hydrogen coordinates ( x $\left.10^{4}\right)$ and isotropic displacement parameters $\left(\AA^{2} \times 10^{3}\right)$ for JF2913FMI.

\begin{tabular}{lcccc}
\hline & $\mathrm{x}$ & $\mathrm{y}$ & $\mathrm{z}$ & $\mathrm{U}(\mathrm{eq})$ \\
& & & & \\
$\mathrm{H}(4 \mathrm{~A})$ & $5570(20)$ & $1298(16)$ & $5272(11)$ & $20(4)$ \\
$\mathrm{H}(4 \mathrm{~B})$ & $7420(20)$ & $1698(16)$ & $5798(12)$ & $22(4)$ \\
$\mathrm{H}(4 \mathrm{C})$ & $7120(20)$ & $266(17)$ & $5294(12)$ & $25(4)$ \\
$\mathrm{H}(5 \mathrm{~A})$ & $9560(20)$ & $596(17)$ & $4100(12)$ & $27(4)$ \\
$\mathrm{H}(5 \mathrm{~B})$ & $9970(20)$ & $1934(17)$ & $4714(13)$ & $31(4)$ \\
$\mathrm{H}(5 \mathrm{C})$ & $9710(20)$ & $2018(17)$ & $3558(13)$ & $27(4)$ \\
$\mathrm{H}(6)$ & $4010(20)$ & $4955(16)$ & $2645(12)$ & $23(4)$ \\
$\mathrm{H}(7)$ & $5330(20)$ & $6759(19)$ & $3577(13)$ & $33(5)$ \\
$\mathrm{H}(8)$ & $7520(20)$ & $6358(18)$ & $4823(13)$ & $31(4)$ \\
$\mathrm{H}(9)$ & $8530(20)$ & $4161(15)$ & $5198(12)$ & $19(4)$ \\
& & & & \\
\hline
\end{tabular}


Table S8. Torsion angles $\left[{ }^{\circ}\right]$ for JF2913FMI.

\begin{tabular}{|c|c|c|c|}
\hline $\mathrm{O}(2)-\mathrm{S}(1)-\mathrm{N}(1)-\mathrm{N}(2)$ & $-68.4(2)$ & $\mathrm{N}(1)-\mathrm{S}(1)-\mathrm{C}(3)-\mathrm{C}(2)$ & $-6.36(8)$ \\
\hline $\mathrm{O}(1)-\mathrm{S}(1)-\mathrm{N}(1)-\mathrm{N}(2)$ & $63.8(2)$ & $\mathrm{O}(2)-\mathrm{S}(1)-\mathrm{C}(3)-\mathrm{C}(6)$ & $60.31(11)$ \\
\hline $\mathrm{C}(3)-\mathrm{S}(1)-\mathrm{N}(1)-\mathrm{N}(2)$ & $177.4(2)$ & $\mathrm{O}(1)-\mathrm{S}(1)-\mathrm{C}(3)-\mathrm{C}(6)$ & $-75.39(11)$ \\
\hline $\mathrm{O}(2)-\mathrm{S}(1)-\mathrm{N}(1)-\mathrm{N}(2 \mathrm{~B})$ & $-65.0(14)$ & $N(1)-S(1)-C(3)-C(6)$ & $172.96(10)$ \\
\hline $\mathrm{O}(1)-\mathrm{S}(1)-\mathrm{N}(1)-\mathrm{N}(2 \mathrm{~B})$ & $67.1(14)$ & $\mathrm{C}(2)-\mathrm{C}(3)-\mathrm{C}(6)-\mathrm{C}(7)$ & $0.36(16)$ \\
\hline $\mathrm{C}(3)-\mathrm{S}(1)-\mathrm{N}(1)-\mathrm{N}(2 \mathrm{~B})$ & $-179.3(14)$ & $\mathrm{S}(1)-\mathrm{C}(3)-\mathrm{C}(6)-\mathrm{C}(7)$ & $-178.89(9)$ \\
\hline $\mathrm{O}(2)-\mathrm{S}(1)-\mathrm{N}(1)-\mathrm{C}(1)$ & $125.60(8)$ & $C(3)-C(6)-C(7)-C(8)$ & $-1.28(17)$ \\
\hline $\mathrm{O}(1)-\mathrm{S}(1)-\mathrm{N}(1)-\mathrm{C}(1)$ & $-102.28(8)$ & $C(6)-C(7)-C(8)-C(9)$ & $0.83(18)$ \\
\hline $\mathrm{C}(3)-\mathrm{S}(1)-\mathrm{N}(1)-\mathrm{C}(1)$ & $11.33(8)$ & $\mathrm{C}(7)-\mathrm{C}(8)-\mathrm{C}(9)-\mathrm{C}(2)$ & $0.61(17)$ \\
\hline $\mathrm{C}(1)-\mathrm{N}(1)-\mathrm{N}(2)-\mathrm{O}(3)$ & $172.4(2)$ & $\mathrm{C}(3)-\mathrm{C}(2)-\mathrm{C}(9)-\mathrm{C}(8)$ & $-1.50(15)$ \\
\hline $\mathrm{S}(1)-\mathrm{N}(1)-\mathrm{N}(2)-\mathrm{O}(3)$ & $6.7(4)$ & $\mathrm{C}(1)-\mathrm{C}(2)-\mathrm{C}(9)-\mathrm{C}(8)$ & $178.68(10)$ \\
\hline $\mathrm{C}(1)-\mathrm{N}(1)-\mathrm{N}(2 \mathrm{~B})-\mathrm{O}(3 \mathrm{~B})$ & $-5(3)$ & & \\
\hline $\mathrm{S}(1)-\mathrm{N}(1)-\mathrm{N}(2 \mathrm{~B})-\mathrm{O}(3 \mathrm{~B})$ & $-172.2(17)$ & & \\
\hline $\mathrm{N}(2)-\mathrm{N}(1)-\mathrm{C}(1)-\mathrm{C}(2)$ & $-178.7(2)$ & & \\
\hline $\mathrm{N}(2 \mathrm{~B})-\mathrm{N}(1)-\mathrm{C}(1)-\mathrm{C}(2)$ & $-179.1(17)$ & & \\
\hline $\mathrm{S}(1)-\mathrm{N}(1)-\mathrm{C}(1)-\mathrm{C}(2)$ & $-12.12(10)$ & & \\
\hline $\mathrm{N}(2)-\mathrm{N}(1)-\mathrm{C}(1)-\mathrm{C}(5)$ & $62.6(2)$ & & \\
\hline $\mathrm{N}(2 \mathrm{~B})-\mathrm{N}(1)-\mathrm{C}(1)-\mathrm{C}(5)$ & $62.2(17)$ & & \\
\hline $\mathrm{S}(1)-\mathrm{N}(1)-\mathrm{C}(1)-\mathrm{C}(5)$ & $-130.84(8)$ & & \\
\hline $\mathrm{N}(2)-\mathrm{N}(1)-\mathrm{C}(1)-\mathrm{C}(4)$ & $-60.9(2)$ & & \\
\hline $\mathrm{N}(2 \mathrm{~B})-\mathrm{N}(1)-\mathrm{C}(1)-\mathrm{C}(4)$ & $-61.2(17)$ & & \\
\hline $\mathrm{S}(1)-\mathrm{N}(1)-\mathrm{C}(1)-\mathrm{C}(4)$ & $105.72(9)$ & & \\
\hline $\mathrm{N}(1)-\mathrm{C}(1)-\mathrm{C}(2)-\mathrm{C}(3)$ & $7.11(12)$ & & \\
\hline$C(5)-C(1)-C(2)-C(3)$ & $124.60(10)$ & & \\
\hline $\mathrm{C}(4)-\mathrm{C}(1)-\mathrm{C}(2)-\mathrm{C}(3)$ & $-109.45(10)$ & & \\
\hline $\mathrm{N}(1)-\mathrm{C}(1)-\mathrm{C}(2)-\mathrm{C}(9)$ & $-173.06(10)$ & & \\
\hline $\mathrm{C}(5)-\mathrm{C}(1)-\mathrm{C}(2)-\mathrm{C}(9)$ & $-55.57(14)$ & & \\
\hline $\mathrm{C}(4)-\mathrm{C}(1)-\mathrm{C}(2)-\mathrm{C}(9)$ & $70.38(13)$ & & \\
\hline $\mathrm{C}(9)-\mathrm{C}(2)-\mathrm{C}(3)-\mathrm{C}(6)$ & $1.05(16)$ & & \\
\hline $\mathrm{C}(1)-\mathrm{C}(2)-\mathrm{C}(3)-\mathrm{C}(6)$ & $-179.11(10)$ & & \\
\hline $\mathrm{C}(9)-\mathrm{C}(2)-\mathrm{C}(3)-\mathrm{S}(1)$ & $-179.63(8)$ & & \\
\hline $\mathrm{C}(1)-\mathrm{C}(2)-\mathrm{C}(3)-\mathrm{S}(1)$ & $0.21(12)$ & & \\
\hline $\mathrm{O}(2)-\mathrm{S}(1)-\mathrm{C}(3)-\mathrm{C}(2)$ & $-119.01(8)$ & & \\
\hline $\mathrm{O}(1)-\mathrm{S}(1)-\mathrm{C}(3)-\mathrm{C}(2)$ & $105.30(9)$ & & \\
\hline
\end{tabular}


Symmetry transformations used to generate equivalent atoms: 
Table S9. Hydrogen bonds for JF2913FMI [ $\AA$ and $\left.{ }^{\circ}\right]$.

\begin{tabular}{lcccc}
\hline $\mathrm{D}-\mathrm{H} \ldots \mathrm{A}$ & $\mathrm{d}(\mathrm{D}-\mathrm{H})$ & $\mathrm{d}(\mathrm{H} \ldots \mathrm{A})$ & $\mathrm{d}(\mathrm{D} \ldots \mathrm{A})$ & $<(\mathrm{DHA})$ \\
\hline $\mathrm{C}(4)-\mathrm{H}(4 \mathrm{~B}) \ldots \mathrm{O}(2) \# 1$ & $0.969(16)$ & $2.530(16)$ & $3.4309(14)$ & $154.8(13)$ \\
$\mathrm{C}(4)-\mathrm{H}(4 \mathrm{C}) \ldots \mathrm{O}\left(3 \mathrm{~B}^{\wedge} \mathrm{b}\right)$ & $0.964(17)$ & $2.531(18)$ & $3.058(7)$ & $114.4(12)$ \\
$\mathrm{C}(5)-\mathrm{H}(5 \mathrm{~A}) \ldots \mathrm{O}\left(3 \mathrm{~B}^{\wedge} \mathrm{b}\right)$ & $0.986(17)$ & $2.385(18)$ & $3.003(7)$ & $120.0(12)$ \\
$\mathrm{C}(6)-\mathrm{H}(6) \ldots \mathrm{O}(1) \# 2$ & $0.956(16)$ & $2.503(16)$ & $3.3121(15)$ & $142.4(13)$ \\
& & & & \\
\hline
\end{tabular}

Symmetry transformations used to generate equivalent atoms:

$\# 1 \mathrm{x}+1 / 2,-\mathrm{y}+1 / 2, \mathrm{z}+1 / 2 \quad \# 2-\mathrm{x}+1 / 2, \mathrm{y}+1 / 2,-\mathrm{z}+1 / 2$

References:

1. Bruker (2014) APEX2 (Version 2014.9) and (2016) SAINT (Version 8.37a). Bruker AXS Inc., Madison, Wisconsin, USA.

2. An Empirical Correction for Absorption Anisotropy, Blessing, R. H. (1995). Acta Cryst., A51, 33-38.

3. Sheldrick, G.M., SADABS (2016) Version 2016/2, 'Siemens Area Detector Absorption Correction' Universität Göttingen: Göttingen, Germany.

4. Sheldrick, G.M., (2002). SHELXTL. Version 6.1. Bruker AXS Inc., Madison, Wisconsin, USA.

5. Sheldrick, G. M., (2014) SHELXT, Universität Göttingen: Göttingen, Germany. Structure determination program. Private communication.

6. Sheldrick, G. M., (2017). SHELXL2017/1. Universität Göttingen: Göttingen, Germany.

Acknowledgment: We thank the National Science Foundation (Grant CHE-0840444) for the Dual Source X-ray diffractometer. 


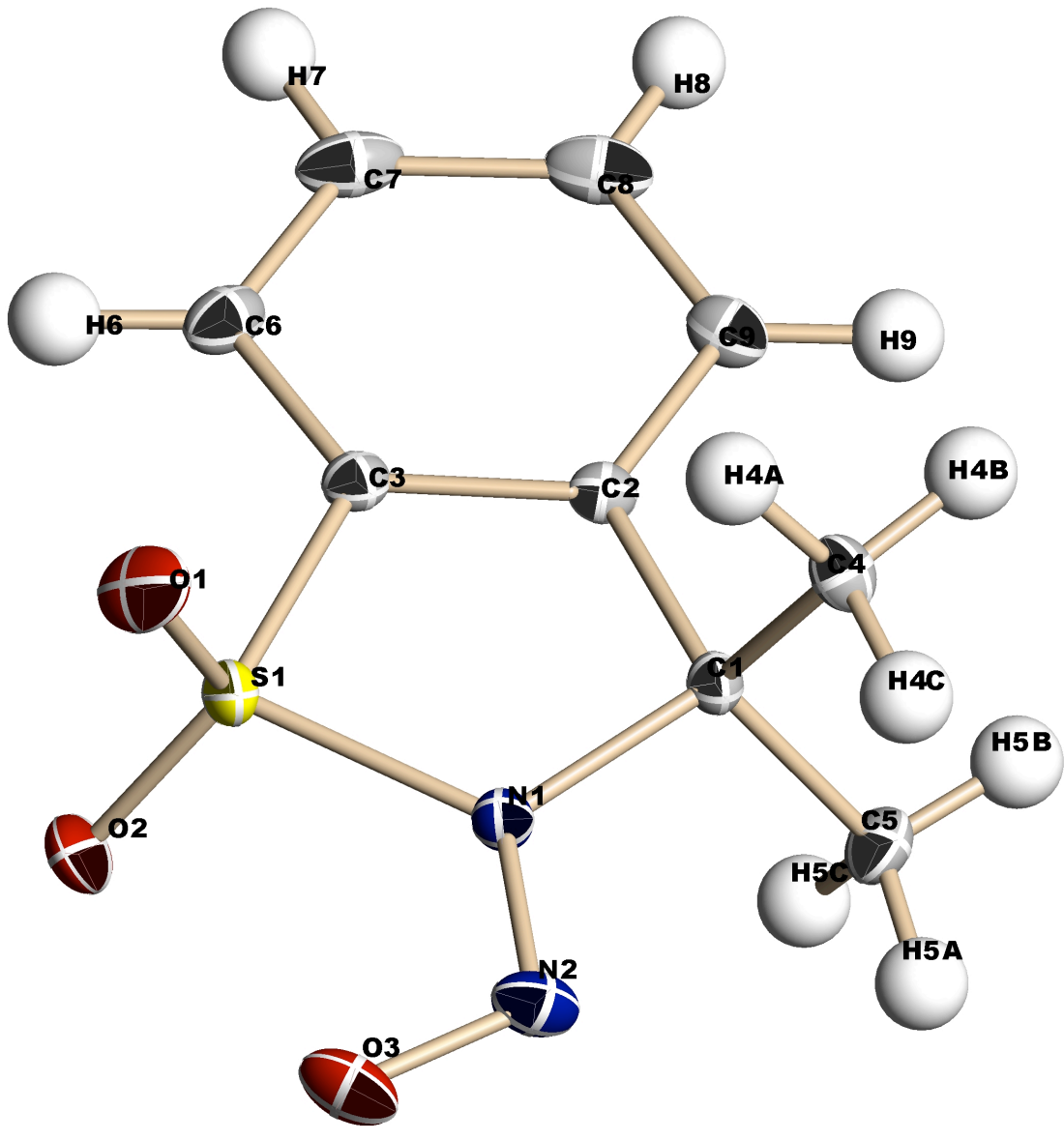

Figure S3. This figure is drawn with 50\% probability ellipsoids 